Supporting Information for

\title{
Redox Switchable Copolymerization of Cyclic Esters and Epoxides by a Zirconium Complex
}

Stephanie M. Quan, Xinke Wang, Rhongjia Zhang, and Paula L. Diaconescu*

Department of Chemistry \& Biochemistry, University of California, Los Angeles 607 Charles E Young Drive East, Los Angeles, CA 90095

\section{Table of Contents}

Tables S1-S5

S2

NMR Spectroscopy

Polymer Precipitation

Low Weight Polymer Analysis

S71

DOSY Experiments

S81

Conversion Studies

S84

Gel Permeation Chromatography

Thermal Gravimetric Analysis

S102

Differential Scanning Calorimetry

S104 
Table S1. Comparison of PLA and PCHO homopolymers and block copolymers.

\begin{tabular}{|c|c|c|c|c|}
\hline Entry & $(\mathrm{co})$ Polymer & $\mathrm{M}_{\mathrm{n}, \mathrm{GPC}^{\mathrm{a}}}$ & PDI & \%PLA \\
\hline 1 & LA & 12.2 & 1.30 & 100 \\
\hline 2 & LA-CHO & 11.4 & 1.32 & 53 \\
\hline 3 & LA-CHO-LA & 16.9 & 1.25 & 69 \\
\hline 4 & CHO & 4.0 & 1.38 & 0 \\
\hline 5 & CHO-LA & 13.9 & 1.66 & 47 \\
\hline 6 & CHO-LA-CHO & 13.3 & 1.53 & 34 \\
\hline
\end{tabular}

${ }^{a} \mathrm{M}_{\mathrm{n}}$ values are reported in $10^{3} \mathrm{~g} / \mathrm{mol}$. Narrow molecular weight polystyrene standards were used for calibration purposes, but reported $M_{n}$ values were not corrected. Samples for entries 2, 3, 5, and 6 were analyzed on a GPC-MALS instrument.

${ }^{\mathrm{b}}$ As determined by ${ }^{1} \mathrm{H}$ NMR spectroscopy of the purified polymer. 


\section{Selective Precipitation of Diblock and Triblock Copolymers}

Procedure for sequential precipitation of copolymers. To isolate the copolymer from any resulting homopolymer fragments selectively, $100 \mathrm{mg}$ of the crude polymer was dissolved in minimal $\mathrm{CH}_{2} \mathrm{Cl}_{2}$ and poured into $10 \mathrm{~mL}$ of cold acetone. The mixture was centrifuged and filtered through a 0.20 micron FTPE filter. The isolated precipitate was dried, dissolved in minimal $\mathrm{CH}_{2} \mathrm{Cl}_{2}$ and poured into $10 \mathrm{~mL}$ of cold hexanes. The mixture was centrifuged and then filtered through a 0.20 micron FTPE filter. Filtrates and precipitates from each precipitation were dried, weighed, and analyzed by ${ }^{1} \mathrm{H}$ NMR spectroscopy and GPC.

Note: All GPC results in this section were obtained on a GPC-MALS instrument.

Table S2. Results of selective precipitation of a PLA-PCHO diblock copolymer as obtained below.

\begin{tabular}{|c|c|c|c|c|}
\hline & PLA : PCHO & Mass $(\mathrm{mg})$ & $\mathrm{M}_{\mathrm{n}}$ & PDI \\
\hline Crude & $1: 0.89$ & 100 & 11.4 & 1.32 \\
\hline Acetone filtrate & $1: 0.33$ & 28 & - & - \\
\hline Acetone precipitate & $1: 0.98$ & 67 & 12.9 & 1.33 \\
\hline Hexanes filtrate & $1: 579$ & 17 & - & - \\
\hline Hexanes precipitate & $1: 0.38$ & 35 & 12.7 & 1.25 \\
\hline
\end{tabular}

Copolymerization of LA and $\mathrm{CHO}$ by (salfan) $\mathrm{Zr}\left(\mathrm{O}^{t} \mathrm{Bu}\right)_{2}$ (red-ox). To a $\mathrm{C}_{6} \mathrm{D}_{6}(0.15 \mathrm{~mL})$ solution of (salfan) $\mathrm{Zr}\left(\mathrm{O}^{t} \mathrm{Bu}\right)_{2}(4.6 \mathrm{mg}, 5 \mu \mathrm{mol})$ in a J-Young NMR tube, a solution of 1,3,5trimethoxybenzene $(16.8 \mathrm{mg}, 50 \mu \mathrm{mol})$ in $\mathrm{C}_{6} \mathrm{D}_{6}(0.15 \mathrm{~mL}), 0.10 \mathrm{~mL}$ of $\mathrm{F}_{2} \mathrm{C}_{6} \mathrm{H}_{4}$ and L-lactide (72.0 mg, $0.500 \mathrm{mmol}$ ) were added. The reaction was heated to $100{ }^{\circ} \mathrm{C}$ and analyzed every 30 min by ${ }^{1} \mathrm{H}$ NMR spectroscopy until completion. A solution of $\left[{ }^{\mathrm{Ac}} \mathrm{Fc}\right]\left[\mathrm{BAr}{ }^{\mathrm{F}} 4\right](5.5 \mathrm{mg}, 5 \mu \mathrm{mol})$ in $\mathrm{F}_{2} \mathrm{C}_{6} \mathrm{H}_{4}(0.10 \mathrm{~mL})$ was then added and the reaction was left at room temperature for $2 \mathrm{~h}$. A solution of cyclohexene oxide $(49.0 \mathrm{mg}, 0.500 \mathrm{mmol})$ in $\mathrm{C}_{6} \mathrm{D}_{6}(0.10 \mathrm{~mL})$ was added. The reaction was heated to $100{ }^{\circ} \mathrm{C}$ and analyzed every $30 \mathrm{~min}$ by ${ }^{1} \mathrm{H}$ NMR spectroscopy until completion. At the end, the reaction mixture was dissolved in $\mathrm{CH}_{2} \mathrm{Cl}_{2}$ and poured into methanol at ambient temperature; a white solid precipitated briefly and was filtered. Yield: $350 \mathrm{mg}, 96.8 \%$. 
Table S3. Results of selective precipitation of a PLA-PCHO-PLA triblock copolymer as obtained below.

\begin{tabular}{|c|c|c|c|c|}
\hline & PLA : PCHO & Mass $(\mathrm{mg})$ & $\mathrm{M}_{\mathrm{n}}$ & PDI \\
\hline Crude & $1: 0.55$ & 100 & 16.9 & 1.25 \\
\hline Acetone filtrate & $1: 0.22$ & 12 & - & - \\
\hline Acetone precipitate & $1: 0.43$ & 69 & 17.4 & 1.23 \\
\hline Hexanes filtrate & $1: 79$ & 5 & - & - \\
\hline Hexanes precipitate & $1: 0.36$ & 56 & 18.1 & 1.38 \\
\hline
\end{tabular}

Copolymerization of LA and CHO by (salfan) $\mathrm{Zr}\left(\mathrm{O}^{t} \mathrm{Bu}\right)_{2}$ (red-ox-red). To a $\mathrm{C}_{6} \mathrm{D}_{6}(0.15 \mathrm{~mL}$ ) solution of (salfan) $\mathrm{Zr}\left(\mathrm{O}^{t} \mathrm{Bu}\right)_{2}(4.6 \mathrm{mg}, 5 \mu \mathrm{mol})$ in a J-Young NMR tube, a solution of 1,3,5trimethoxybenzene $(16.8 \mathrm{mg}, 50 \mu \mathrm{mol})$ in $\mathrm{C}_{6} \mathrm{D}_{6}(0.15 \mathrm{~mL}), 0.10 \mathrm{~mL}$ of $\mathrm{F}_{2} \mathrm{C}_{6} \mathrm{H}_{4}$ and L-lactide $(72.0 \mathrm{mg}, 0.500 \mathrm{mmol})$ were added. The reaction was heated to $100{ }^{\circ} \mathrm{C}$ and analyzed every 30 min by ${ }^{1} \mathrm{H}$ NMR spectroscopy until completion. A solution of [ $\left.{ }^{\mathrm{Ac}} \mathrm{Fc}\right]\left[\mathrm{BAr}{ }_{4}{ }_{4}\right](5.5 \mathrm{mg}, 5 \mu \mathrm{mol})$ in $\mathrm{F}_{2} \mathrm{C}_{6} \mathrm{H}_{4}(0.10 \mathrm{~mL})$ was then added and the reaction was left at room temperature for $2 \mathrm{~h}$. A solution of cyclohexene oxide $(49.0 \mathrm{mg}, 0.500 \mathrm{mmol})$ in $\mathrm{C}_{6} \mathrm{D}_{6}(0.10 \mathrm{~mL})$ was added. The reaction was heated to $100{ }^{\circ} \mathrm{C}$ and analyzed every $30 \mathrm{~min}$ by ${ }^{1} \mathrm{H}$ NMR spectroscopy until completion. A solution of $\mathrm{CoCp}_{2}(5.5 \mathrm{mg}, 5 \mu \mathrm{mol})$ in $\mathrm{C}_{6} \mathrm{D}_{6}(0.10 \mathrm{~mL})$ was then added and the reaction was left at room temperature for $2 \mathrm{~h}$. L-lactide $(72.0 \mathrm{mg}, 0.500 \mathrm{mmol})$ was added. The reaction was heated to $100{ }^{\circ} \mathrm{C}$ and analyzed every 30 min by ${ }^{1} \mathrm{H}$ NMR spectroscopy until completion. At the end, the reaction mixture was dissolved in $\mathrm{CH}_{2} \mathrm{Cl}_{2}$ and poured into methanol at ambient temperature; a white solid precipitated briefly and was filtered. Yield: $350 \mathrm{mg}, 96.8 \%$. 
Table S4. Results of selective precipitation of a PCHO-PLA diblock copolymer as obtained below.

\begin{tabular}{|c|c|c|c|c|}
\hline & PLA : PCHO & Mass (mg) & $\mathrm{M}_{\mathrm{n}}$ & PDI \\
\hline Crude & $1: 1.15$ & 100 & 13.8 & 1.66 \\
\hline Acetone filtrate & $1: 0.40$ & 25 & - & - \\
\hline Acetone precipitate & $1: 1.38$ & 68 & 18.6 & 1.48 \\
\hline Hexanes filtrate & $1: 145$ & 6 & - & - \\
\hline Hexanes precipitate & $1: 1.29$ & 47 & 15.8 & 1.60 \\
\hline
\end{tabular}

Copolymerization of LA and CHO by [(salfan) $\left.\mathrm{Zr}\left(\mathrm{O}^{t} \mathrm{Bu}\right)_{2}\right]\left[\mathrm{BAr}^{\mathrm{F}} 4\right]$ (ox-red). To a $\mathrm{C}_{6} \mathrm{D}_{6}(0.15$ $\mathrm{mL}$ ) solution of (salfan) $\mathrm{Zr}\left(\mathrm{O}^{t} \mathrm{Bu}\right)_{2}(4.6 \mathrm{mg}, 5 \mu \mathrm{mol})$ in a J-Young NMR tube, a solution of 1,3,5trimethoxybenzene $(16.8 \mathrm{mg}, 50 \mu \mathrm{mol})$ in $\mathrm{C}_{6} \mathrm{D}_{6}(0.15 \mathrm{~mL}), 0.10 \mathrm{~mL}$ of $\mathrm{F}_{2} \mathrm{C}_{6} \mathrm{H}_{4}$ and a solution of $\left[{ }^{\mathrm{Ac}} \mathrm{Fc}\right]\left[\mathrm{BAr}_{4} \mathrm{~F}_{4}\right](5.5 \mathrm{mg}, 5 \mu \mathrm{mol})$ in $\mathrm{F}_{2} \mathrm{C}_{6} \mathrm{H}_{4}(0.10 \mathrm{~mL})$ were added and the reaction was left at room temperature for $2 \mathrm{~h}$. A solution of cyclohexene oxide $(49.0 \mathrm{mg}, 0.500 \mathrm{mmol})$ in $\mathrm{C}_{6} \mathrm{D}_{6}(0.10$ $\mathrm{mL}$ ) was added. The reaction was heated to $100{ }^{\circ} \mathrm{C}$ and analyzed every $30 \mathrm{~min}$ by ${ }^{1} \mathrm{H}$ NMR spectroscopy until completion. A solution of $\mathrm{CoCp}_{2}(5.5 \mathrm{mg}, 5 \mu \mathrm{mol})$ in $\mathrm{C}_{6} \mathrm{D}_{6}(0.10 \mathrm{~mL})$ was then added and the reaction was left at room temperature for $2 \mathrm{~h}$. A $\mathrm{C}_{6} \mathrm{D}_{6}$ solution of L-lactide solution $(72.0 \mathrm{mg}, 0.500 \mathrm{mmol})$ was added. After being heated at $100{ }^{\circ} \mathrm{C}$ for $2 \mathrm{~h}$, the reaction was monitored by ${ }^{1} \mathrm{H}$ NMR spectroscopy until completion. At the end, the reaction was dissolved in $\mathrm{CH}_{2} \mathrm{Cl}_{2}$ and poured into methanol at ambient temperature; a white solid precipitated briefly and was filtered. Yield: $280 \mathrm{mg}, 91.3 \%$. 
Table S5. Results of selective precipitation of a PCHO-PLA-PCHO triblock copolymer as obtained below.

\begin{tabular}{|c|c|c|c|c|}
\hline & PLA : PCHO & Mass $(\mathrm{mg})$ & $\mathrm{M}_{\mathrm{n}}$ & PDI \\
\hline Crude & $1: 1.99$ & 100 & 13.3 & 1.53 \\
\hline Acetone filtrate & $1: 0.23$ & Trace & - & - \\
\hline Acetone precipitate & $1: 2.41$ & 99 & 14.0 & 1.47 \\
\hline Hexanes filtrate & $1: 214$ & 17 & - & - \\
\hline Hexanes precipitate & $1: 1.43$ & 59 & 16.8 & 1.49 \\
\hline
\end{tabular}

Copolymerization of LA and CHO by [(salfan) $\left.\mathrm{Zr}\left(\mathrm{O}^{t} \mathrm{Bu}\right)_{2}\right]\left[\mathrm{BAr}^{\mathrm{F}_{4}}\right.$ ] (ox-red-ox). To a $\mathrm{C}_{6} \mathrm{D}_{6}(0.15$ $\mathrm{mL}$ ) solution of (salfan) $\mathrm{Zr}\left(\mathrm{O}^{t} \mathrm{Bu}\right)_{2}(4.6 \mathrm{mg}, 5 \mu \mathrm{mol})$ in a J-Young NMR tube, a solution of 1,3,5trimethoxybenzene $(16.8 \mathrm{mg}, 50 \mu \mathrm{mol})$ in $\mathrm{C}_{6} \mathrm{D}_{6}(0.15 \mathrm{~mL}), 0.10 \mathrm{~mL} \mathrm{~F}_{2} \mathrm{C}_{6} \mathrm{H}_{4}$ and a solution of $\left[{ }^{\mathrm{Ac}} \mathrm{Fc}\right]\left[\mathrm{BAr}_{4} \mathrm{~F}_{4}\right](5.5 \mathrm{mg}, 5 \mu \mathrm{mol})$ in $\mathrm{F}_{2} \mathrm{C}_{6} \mathrm{H}_{4}(0.10 \mathrm{~mL})$ was added and the reaction was left at room temperature for $2 \mathrm{~h}$. A solution of cyclohexene oxide $(49.0 \mathrm{mg}, 0.500 \mathrm{mmol})$ in $\mathrm{C}_{6} \mathrm{D}_{6}(0.10 \mathrm{~mL})$ was added. The reaction was heated to $100{ }^{\circ} \mathrm{C}$ and analyzed every 30 min by ${ }^{1} \mathrm{H}$ NMR spectroscopy until completion. A solution of $\mathrm{CoCp}_{2}(5.5 \mathrm{mg}, 5 \mu \mathrm{mol})$ in $\mathrm{C}_{6} \mathrm{D}_{6}(0.10 \mathrm{~mL})$ was then added and the reaction was left at room temperature for $2 \mathrm{~h} . \mathrm{A} \mathrm{C}_{6} \mathrm{D}_{6}$ solution of L-lactide solution (72.0 mg, $0.500 \mathrm{mmol}$ ) was added. After being heated at $100{ }^{\circ} \mathrm{C}$ for $2 \mathrm{~h}$, the reaction was monitored by ${ }^{1} \mathrm{H}$ NMR spectroscopy until completion. A solution of $\left[{ }^{\mathrm{Ac}} \mathrm{Fc}\right]\left[\mathrm{BAr}{ }_{4}\right](5.5 \mathrm{mg}, 5$ $\mu \mathrm{mol})$ in $\mathrm{F}_{2} \mathrm{C}_{6} \mathrm{H}_{4}(0.10 \mathrm{~mL})$ was then added and the reaction was left at room temperature for $2 \mathrm{~h}$. A solution of cyclohexene oxide $(49.0 \mathrm{mg}, 0.500 \mathrm{mmol})$ in $\mathrm{C}_{6} \mathrm{D}_{6}(0.10 \mathrm{~mL})$ was added. After being heated at $100{ }^{\circ} \mathrm{C}$ for $2 \mathrm{~h}$, the reaction was monitored by ${ }^{1} \mathrm{H}$ NMR spectroscopy until completion. At the end, the reaction was dissolved in $\mathrm{CH}_{2} \mathrm{Cl}_{2}$ and poured into methanol at ambient temperature; a white solid precipitated briefly and was filtered. Yield: $280 \mathrm{mg}, 91.3 \%$. 


\section{${ }^{1}$ H NMR Spectroscopy}

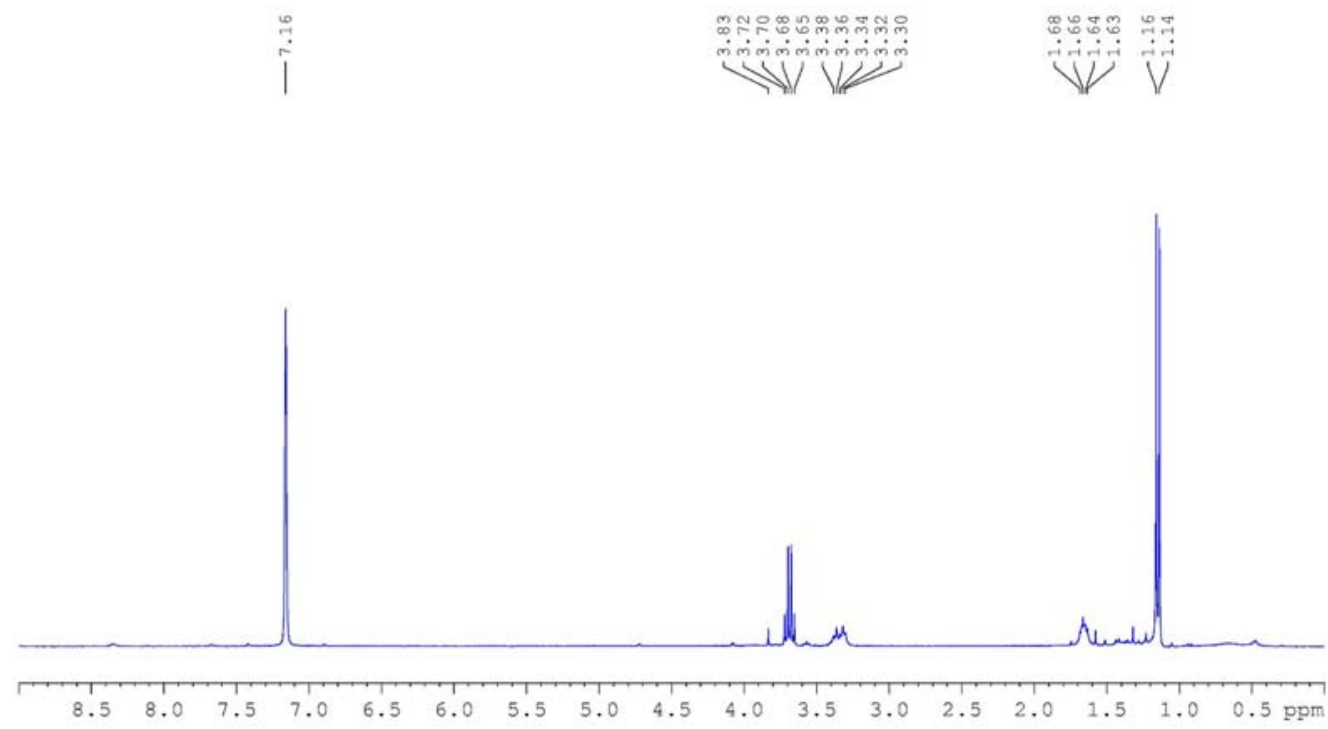

Figure S1. Polymerization of THF by in situ generated [(salfan) $\left.\mathrm{Zr}\left(\mathrm{O}^{t} \mathrm{Bu}\right)_{2}\right]\left[\mathrm{BAr}{ }^{\mathrm{F}}{ }_{4}\right] .{ }^{1} \mathrm{H} \mathrm{NMR}$ $\left(300 \mathrm{MHz}, 25{ }^{\circ} \mathrm{C}, \mathrm{C}_{6} \mathrm{D}_{6}\right) \delta(\mathrm{ppm}): 3.69$ (m, 4H, OCH $\left.2 \mathrm{THF}\right), 3.33$ (m, 4H, OCH2 PTHF), 1.65 (m, 4H, OCH2 $\left.\mathrm{CH}_{2} \mathrm{PTHF}\right), 1.15$ (m, OCH2CH $\left.2, \mathrm{THF}\right)$.

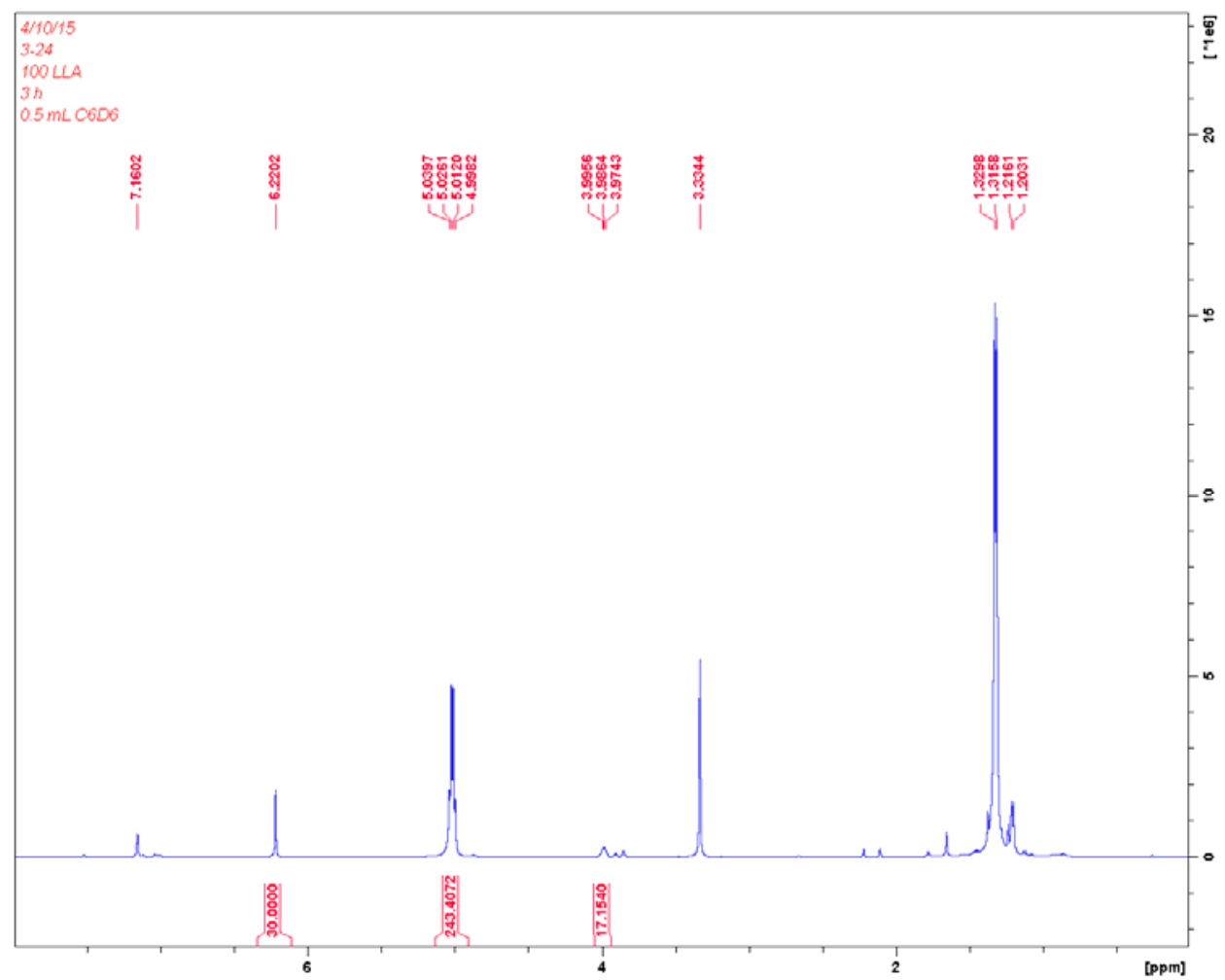

Figure S2. Polymerization of L-lactide by (salfan) $\mathrm{Zr}\left(\mathrm{O}^{t} \mathrm{Bu}\right)_{2} .{ }^{1} \mathrm{H} \mathrm{NMR}\left(300 \mathrm{MHz}, 25{ }^{\circ} \mathrm{C}, \mathrm{C}_{6} \mathrm{D}_{6}\right)$, $\delta$ (ppm): 6.22 (s, 3H, $\mathrm{PhH} \mathrm{TMB),} 5.02$ (q, 2H, $\left.\mathrm{CHCH}_{3} \mathrm{PLA}\right), 3.99$ (q, 2H, $\left.\mathrm{CHCH}_{3} \mathrm{LLA}\right), 3.33$ (s, 9H, $\left.\mathrm{CH}_{3} \mathrm{TMB}\right), 1.32$ (d, 6H, $\left.\mathrm{CHCH}_{3} \mathrm{PLA}\right), 1.20$ (d, 6H, CHCH3 LLA). 


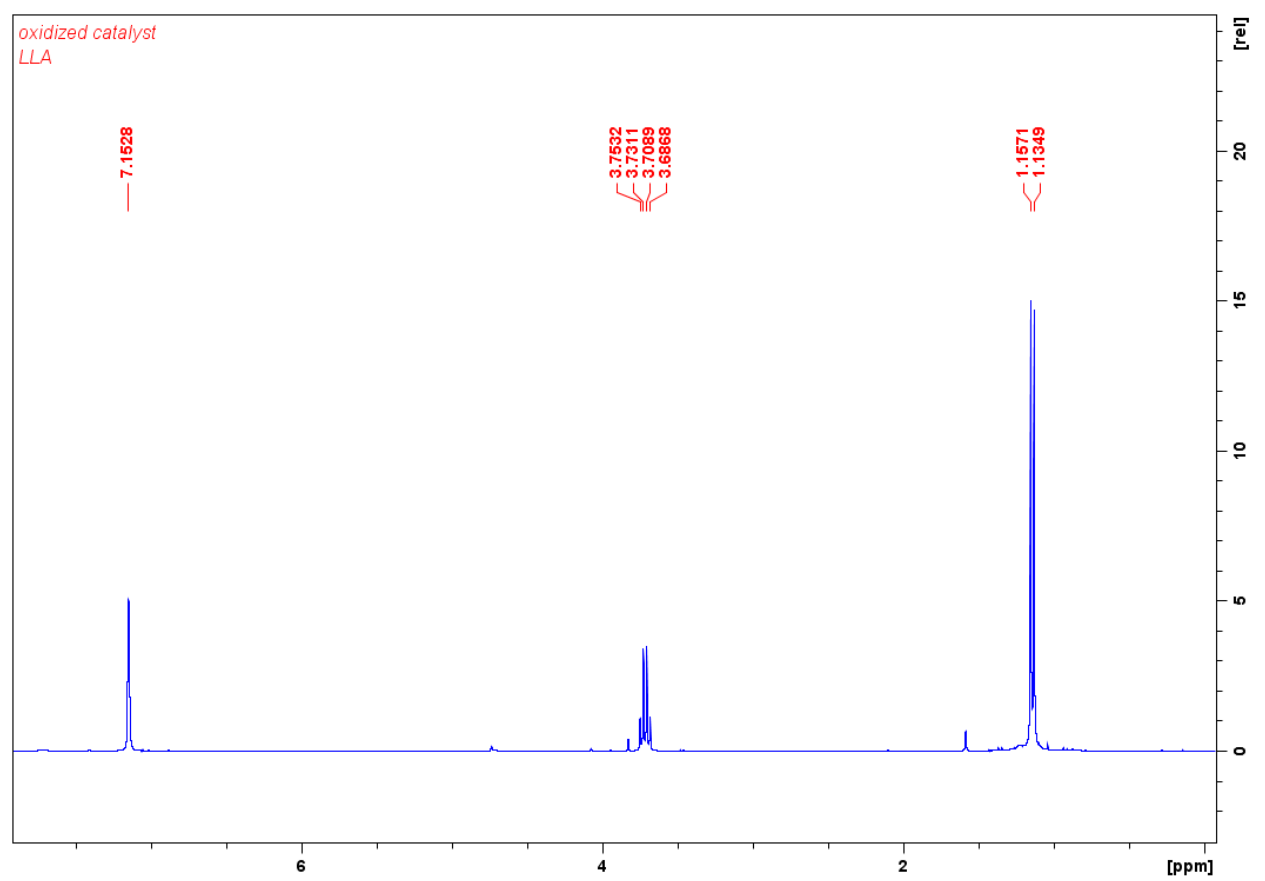

Figure S3. Polymerization of L-lactide by [(salfan) $\left.\mathrm{Zr}\left(\mathrm{O}^{t} \mathrm{Bu}\right)_{2}\right]\left[\mathrm{BAr}^{\mathrm{F}}{ }_{4}\right] .{ }^{1} \mathrm{H}$ NMR $(300 \mathrm{MHz}, 25$ ${ }^{\circ} \mathrm{C}, \mathrm{C}_{6} \mathrm{D}_{6}$ ), $\delta$ (ppm): 3.72 (q, 2H, $\left.\mathrm{CHCH}_{3} \mathrm{LA}\right), 1.14$ (d, 6H, $\mathrm{CHCH}_{3} \mathrm{LA}$ ).

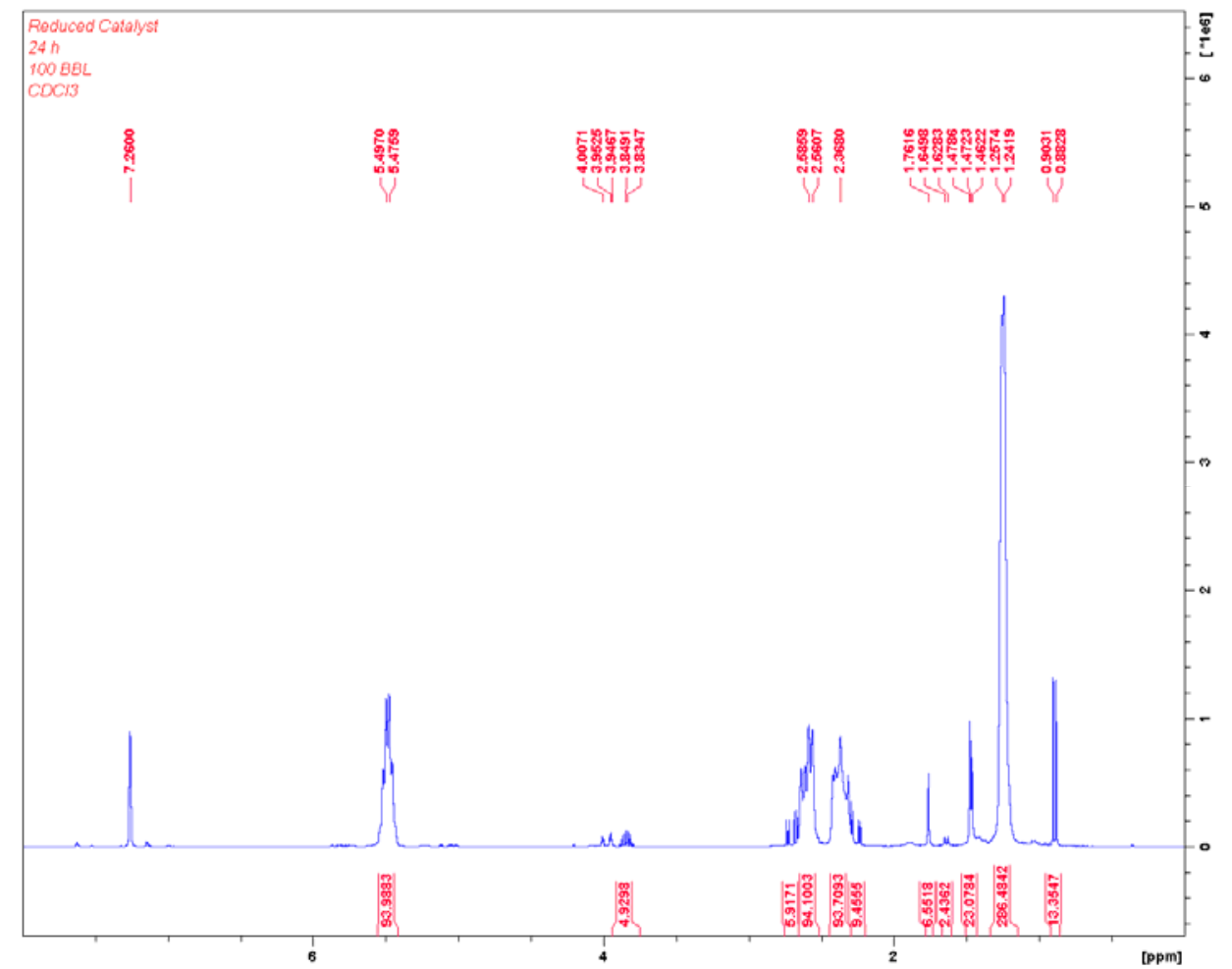

Figure S4. Polymerization of $\beta$-butyrolactone by (salfan) $\mathrm{Zr}\left(\mathrm{O}^{t} \mathrm{Bu}\right)_{2} .{ }^{1} \mathrm{H} \mathrm{NMR}\left(300 \mathrm{MHz}, 25{ }^{\circ} \mathrm{C}\right.$, $\left.\mathrm{CDCl}_{3}\right), \delta(\mathrm{ppm}): 5.48\left(\mathrm{~m}, 1 \mathrm{H}, \mathrm{OCHCH}_{3} \mathrm{PHB}\right), 3.84\left(\mathrm{~m}, 1 \mathrm{H}, \mathrm{OCHCH}_{3}, \mathrm{BBL}\right), 2.58-2.37(\mathrm{~m}, 2 \mathrm{H}$, $\mathrm{COCH}_{2} \mathrm{PHB}$ ), 1.25 (t, 3H, $\left.\mathrm{OCHCH}_{3} \mathrm{PHB}\right), 0.89$ (t, 3H, $\mathrm{OCHCH}_{3} \mathrm{BBL}$ ). 


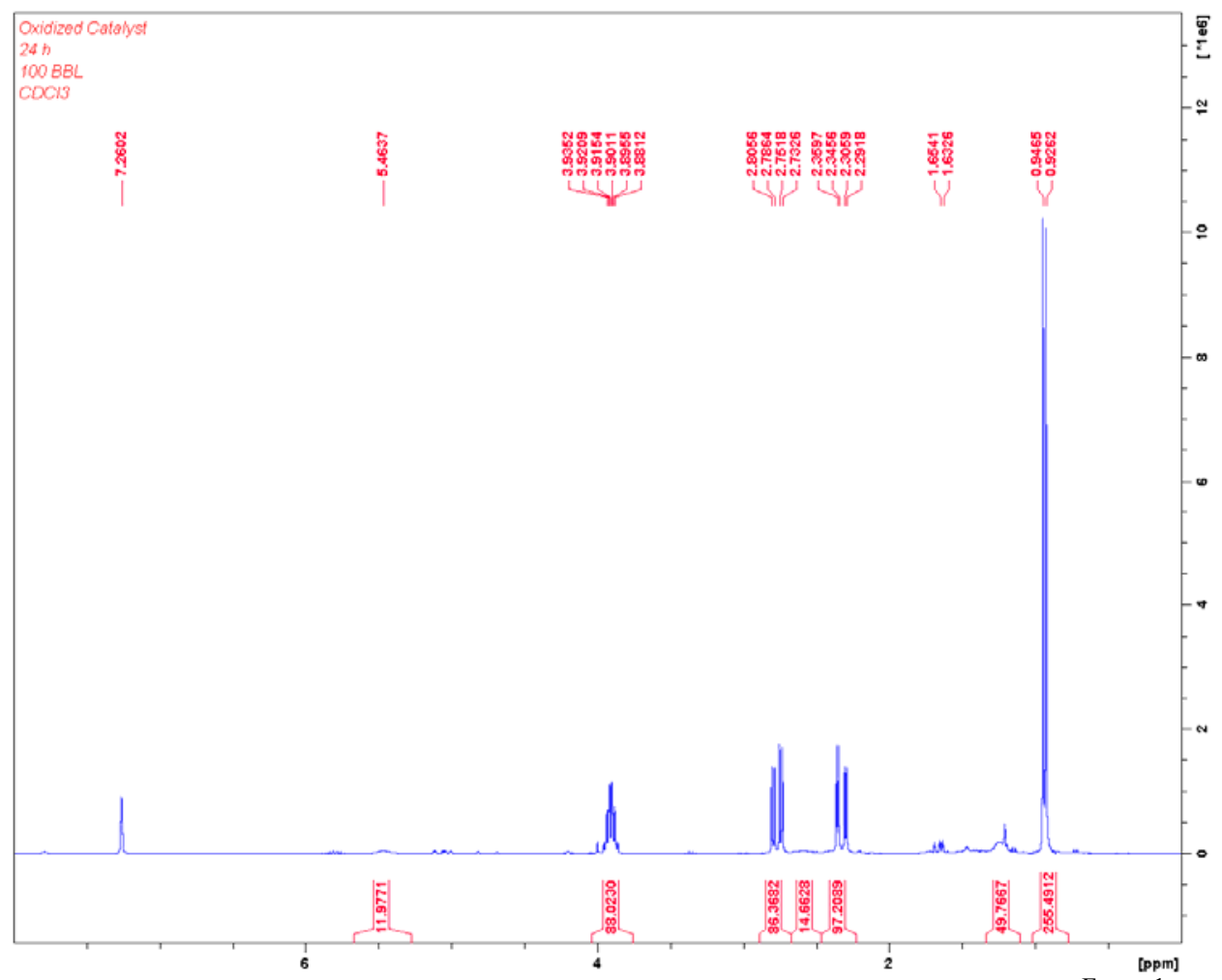

Figure S5. Polymerization of $\beta$-butyrolactone by $\left[(\operatorname{salfan}) \mathrm{Zr}\left(\mathrm{O}^{t} \mathrm{Bu}\right)_{2}\right]\left[\mathrm{BAr}^{\mathrm{F}}{ }^{\mathrm{F}}\right] .{ }^{\mathrm{g}}{ }^{\mathrm{b} m} \mathrm{H}$ NMR $(300$ $\left.\mathrm{MHz}, 25^{\circ} \mathrm{C}, \mathrm{CDCl}_{3}\right), \delta(\mathrm{ppm}): 5.46\left(\mathrm{~m}, 1 \mathrm{H}, \mathrm{OCHCH}_{3} \mathrm{PHB}\right), 3.90\left(\mathrm{~m}, 1 \mathrm{H}, \mathrm{OCHCH}_{3}, \mathrm{BBL}\right)$, 2.77-2.32 (m, 2H, $\left.\mathrm{COCH}_{2} \mathrm{BBL}\right), 0.93\left(\mathrm{t}, 3 \mathrm{H}, \mathrm{OCHCH}_{3} \mathrm{BBL}\right)$.

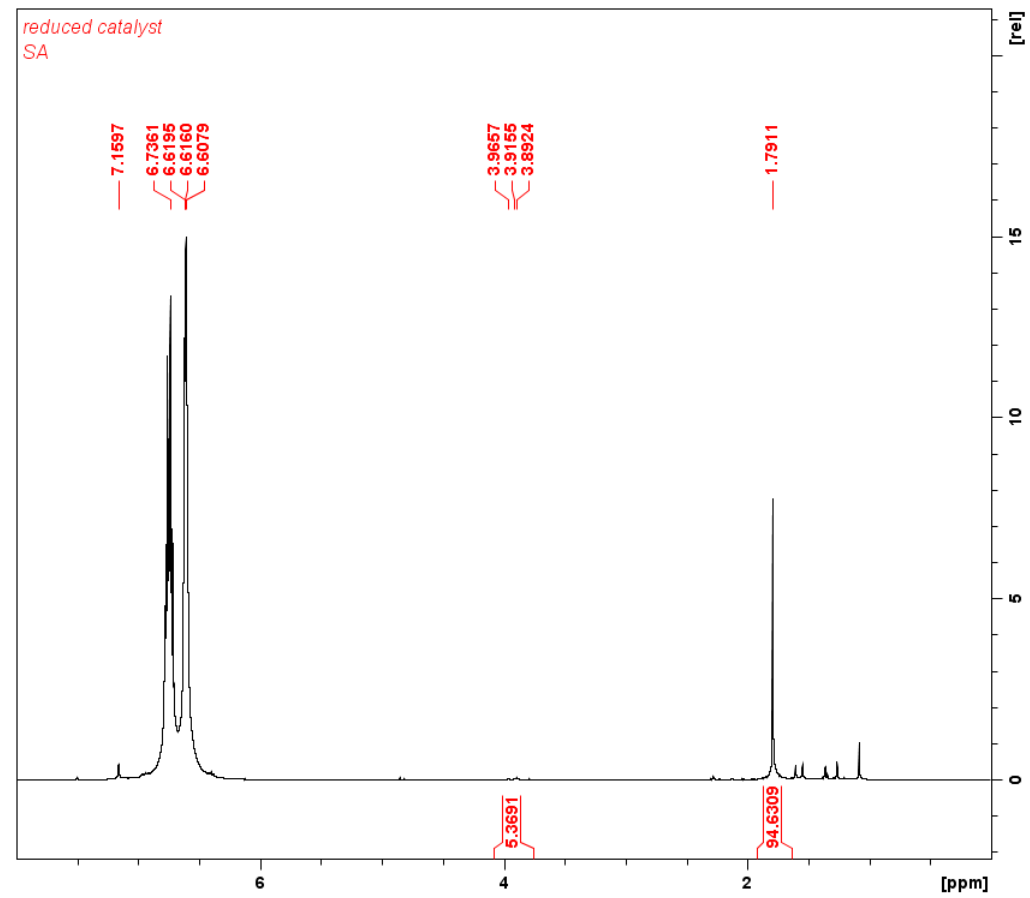

Figure S6. Polymerization of succinic anhydride by (salfan) $\mathrm{Zr}\left(\mathrm{O}^{t} \mathrm{Bu}\right)_{2} .{ }^{1} \mathrm{H}$ NMR $(300 \mathrm{MHz}, 25$ $\left.{ }^{\circ} \mathrm{C}, \mathrm{C}_{6} \mathrm{D}_{6}\right), \delta(\mathrm{ppm}): 6.74$ ( $\mathrm{CFCH}$ 1,2-difluorobenzene), 6.62 (CFCHCH 1,2-difluorobenzene), $3.91\left(\mathrm{~m}, \mathrm{CH}_{2}, \mathrm{PSA}\right), 1.79$ (s, $\left.\mathrm{CH}_{2}, \mathrm{SA}\right)$. 


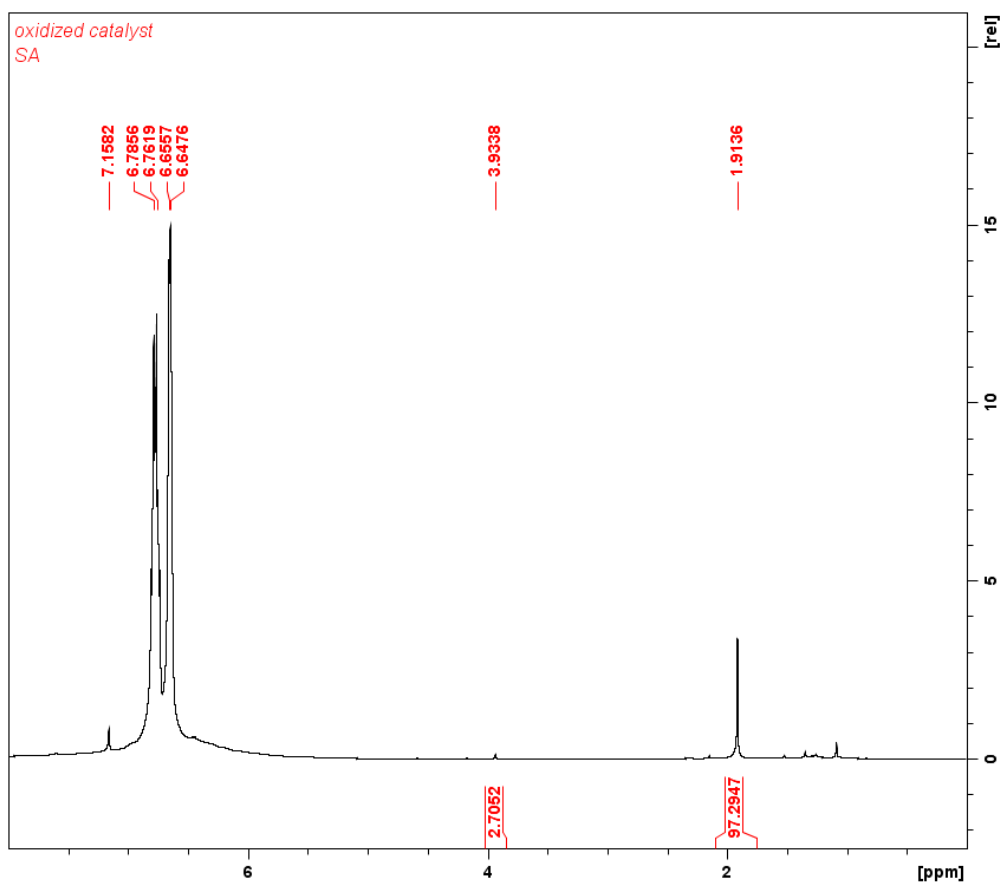

Figure S7. Polymerization of succinic anhydride by [(salfan $\left.) \mathrm{Zr}\left(\mathrm{O}^{t} \mathrm{Bu}\right)_{2}\right]\left[\mathrm{BAr}{ }^{\mathrm{F}}{ }_{4}\right] .{ }^{1} \mathrm{H} \mathrm{NMR}(300$ $\left.\mathrm{MHz}, 25{ }^{\circ} \mathrm{C}, \mathrm{C}_{6} \mathrm{D}_{6}\right), \delta$ (ppm): 6.74 ( $\mathrm{CFCH}$ 1,2-difluorobenzene), 6.62 (CFCHCH 1,2difluorobenzene), 3.91 ( $\mathrm{m}, \mathrm{CH}_{2}$, PSA), 1.79 (s, $\left.\mathrm{CH}_{2}, \mathrm{SA}\right)$.

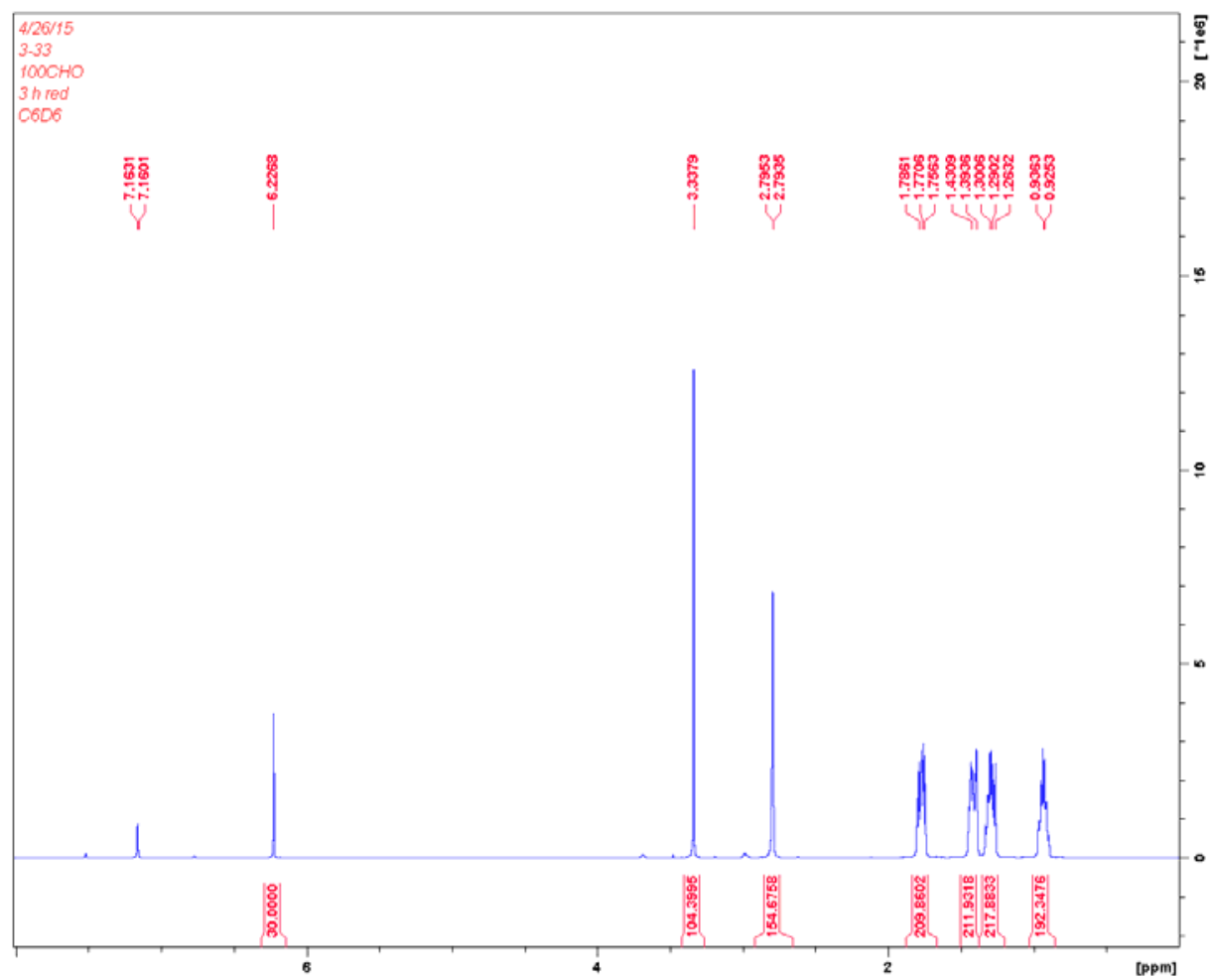

Figure S8. Polymerization of cyclohexene oxide by (salfan) $\mathrm{Zr}\left(\mathrm{O}^{t} \mathrm{Bu}\right){ }_{2} .{ }^{1} \mathrm{H}$ NMR $(300 \mathrm{MHz}, 25$ ${ }^{\circ} \mathrm{C}, \mathrm{C}_{6} \mathrm{D}_{6}$ ), $\delta$ (ppm): 6.22 (s, 3H, PhH TMB), 3.33 (s, 9H, $\mathrm{CH}_{3} \mathrm{TMB}$ ), 2.79 (s, 2H, COCH CHO), $1.77\left(\mathrm{~m}, 2 \mathrm{H}, \mathrm{COCHCH}_{2} \mathrm{CHO}\right), 1.39\left(\mathrm{~m}, 2 \mathrm{H}, \mathrm{COCHCH}_{2} \mathrm{CHO}\right), 1.29\left(\mathrm{~m}, 2 \mathrm{H}, \mathrm{COCHCH}_{2} \mathrm{CH}_{2}\right.$ $\mathrm{CHO}), 0.93\left(\mathrm{~m}, 2 \mathrm{H}, \mathrm{COCHCH}_{2} \mathrm{CH}_{2} \mathrm{CHO}\right)$. 


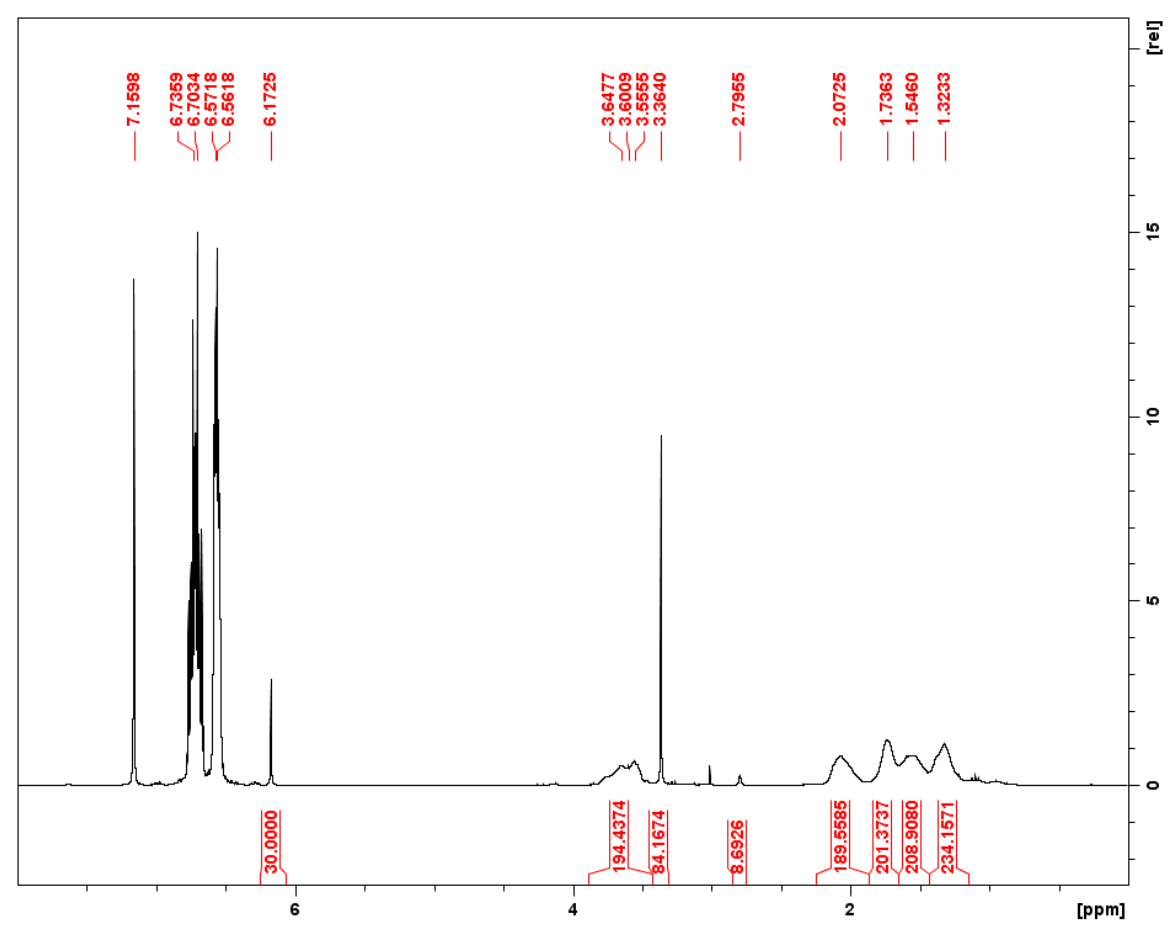

Figure S9. Polymerization of cyclohexene oxide by [(salfan $\left.) \mathrm{Zr}\left(\mathrm{O}^{t} \mathrm{Bu}\right)_{2}\right]\left[\mathrm{BAr}{ }^{\mathrm{F}} 4\right] .{ }^{1} \mathrm{H}$ NMR $(300$ $\left.\mathrm{MHz}, 25{ }^{\circ} \mathrm{C}, \mathrm{C}_{6} \mathrm{D}_{6}\right), \delta(\mathrm{ppm}): 6.72$ (CFCH 1,2-difluorobenzene), 6.57 (CFCHCH 1,2difluorobenzene), 6.17 (s, 3H, $\mathrm{PhH} \mathrm{TMB}$ ), 3.60 (m, 2H, COCH PCHO), 3.36 (s, 9H, CH3 TMB), 2.80 (s, 2H, COCH CHO), 2.07 (m, 2H, $\left.\mathrm{COCHCH}_{2} \mathrm{PCHO}\right), 1.74$ (m, 2H, $\mathrm{COCHCH}_{2} \mathrm{PCHO}$ ), $1.54\left(\mathrm{~m}, 2 \mathrm{H}, \mathrm{COCHCH}_{2} \mathrm{CH}_{2} \mathrm{PCHO}\right), 1.32$ (m, 2H, $\left.\mathrm{COCHCH}_{2} \mathrm{CH}_{2} \mathrm{PCHO}\right)$.

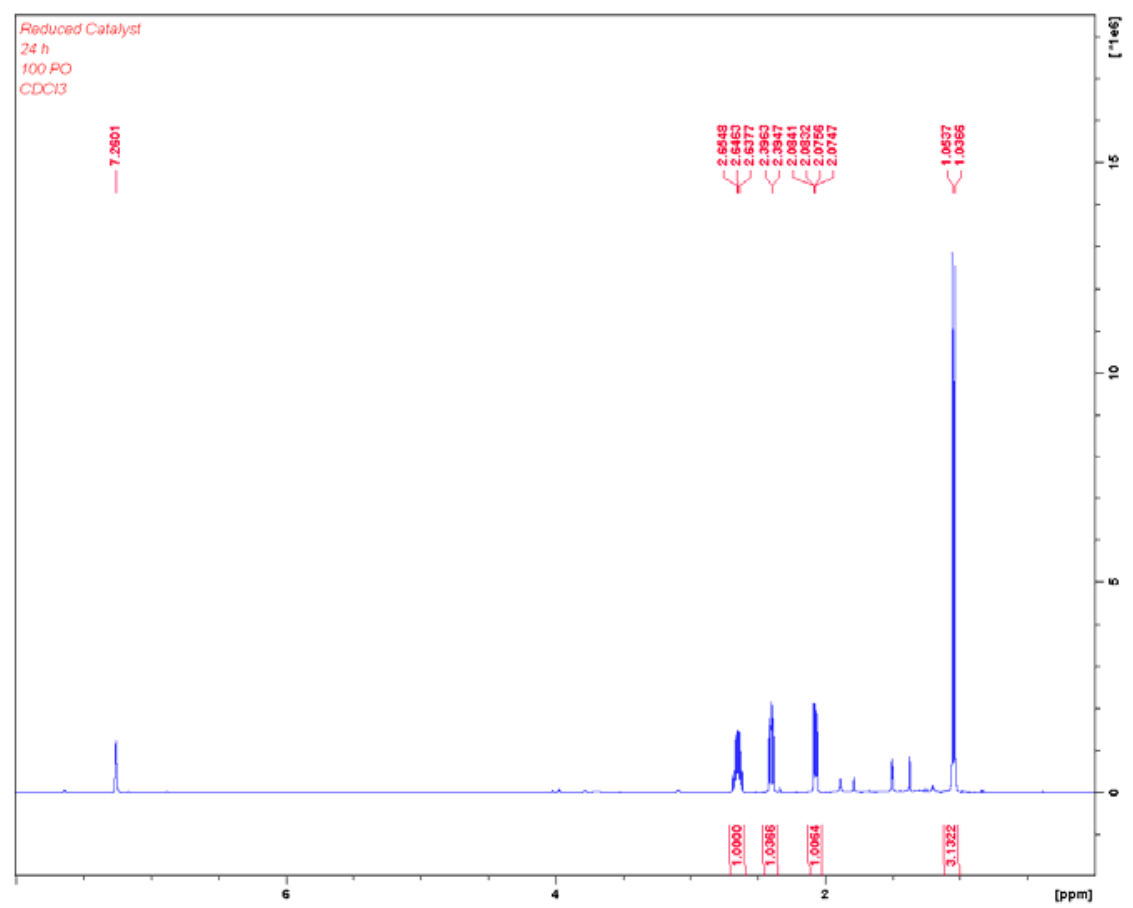

Figure S10. Table 1, entry 9. Polymerization of propylene oxide by (salfan) $\mathrm{Zr}\left(\mathrm{O}^{t} \mathrm{Bu}\right)_{2} .{ }^{1} \mathrm{H} \mathrm{NMR}$ $\left(300 \mathrm{MHz}, 25^{\circ} \mathrm{C}, \mathrm{C}_{6} \mathrm{D}_{6}\right), \delta(\mathrm{ppm}): 2.65$ (m, 1H, $\left.\mathrm{COCHCH}_{3} \mathrm{PO}\right), 2.39$ (m, 1H, $\left.\mathrm{COCH}_{2} \mathrm{PO}\right), 2.07$ (m, $\left.1 \mathrm{H}, \mathrm{COCH}_{2} \mathrm{PO}\right), 1.04\left(\mathrm{~d}, 3 \mathrm{H}, \mathrm{COCHCH}_{3} \mathrm{PO}\right)$. 


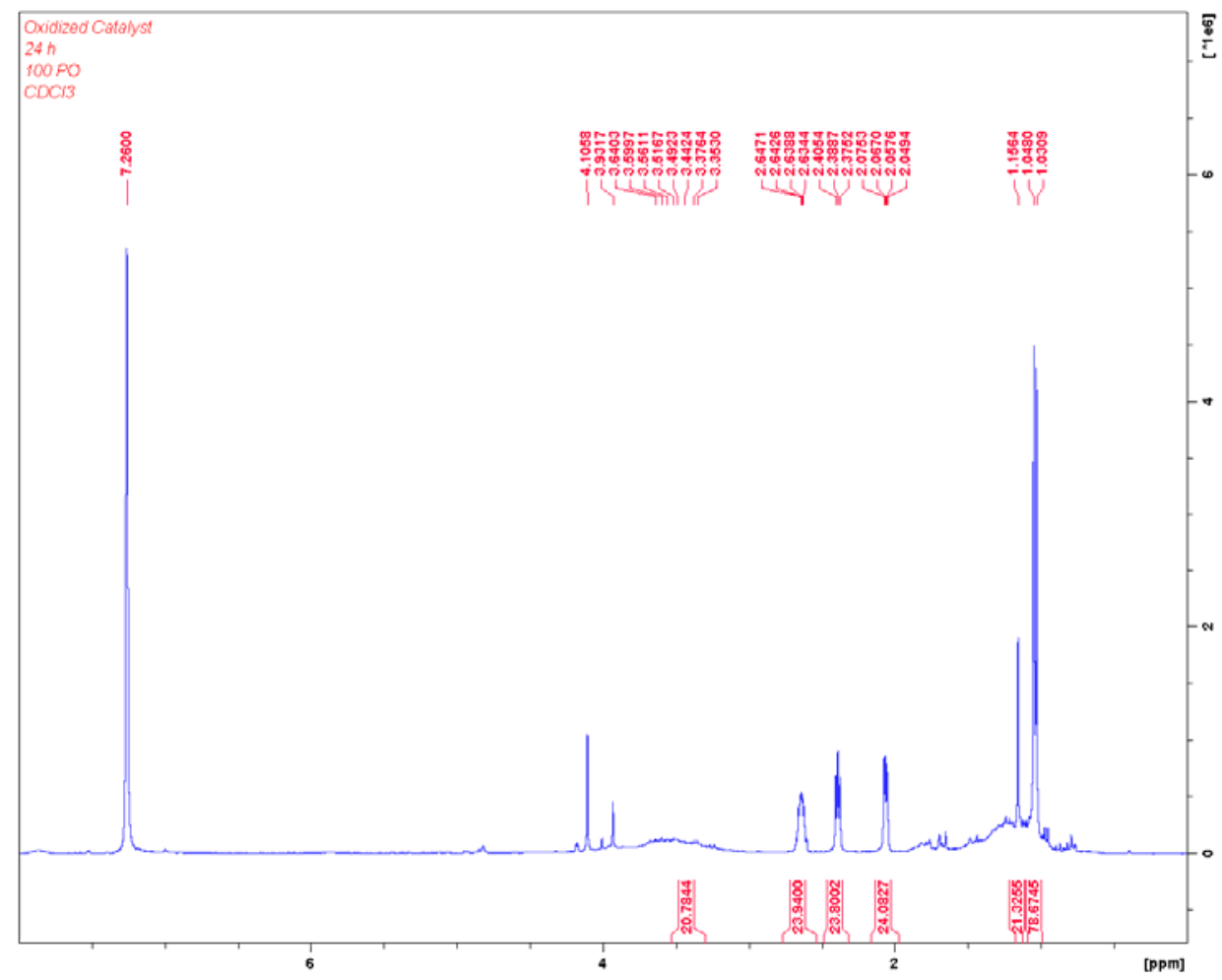

Figure S11. Table 1, entry 10. Polymerization of propylene oxide by [(salfan) $\left.\mathrm{Zr}\left(\mathrm{O}^{t} \mathrm{Bu}\right)_{2}\right]\left[\mathrm{BAr}{ }_{4}\right]$. ${ }^{1} \mathrm{H}$ NMR $\left(300 \mathrm{MHz}, 25{ }^{\circ} \mathrm{C}, \mathrm{CDCl}_{3}\right), \delta(\mathrm{ppm}): 3.49\left(\mathrm{~m}, 1 \mathrm{H}, \mathrm{COCHCH}_{3} \mathrm{PPO}\right), 2.64(\mathrm{~m}, 1 \mathrm{H}$, $\left.\mathrm{COCHCH}_{3} \mathrm{PO}\right), 2.38\left(\mathrm{~m}, 1 \mathrm{H}, \mathrm{COCH}_{2} \mathrm{PO}\right), 2.06\left(\mathrm{~m}, 1 \mathrm{H}, \mathrm{COCH}_{2} \mathrm{PO}\right), 1.56\left(\mathrm{~d}, 3 \mathrm{H}, \mathrm{COCHCH}_{3}\right.$ PPO), 1.04 (d, 3H, $\left.\mathrm{COCHCH}_{3} \mathrm{PO}\right)$.

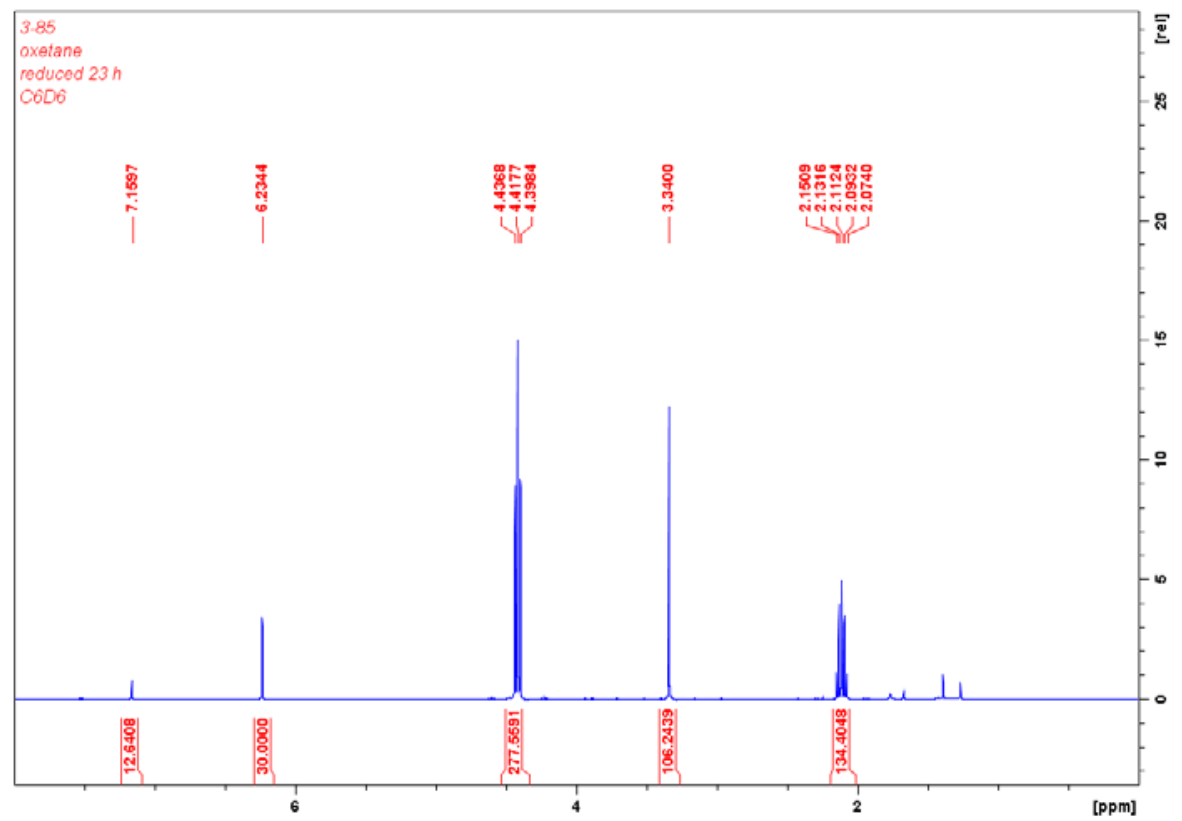

Figure S12. Table 1, entry 11. Polymerization of oxetane by (salfan) $\mathrm{Zr}\left(\mathrm{O}^{t} \mathrm{Bu}\right) 2 .{ }^{1} \mathrm{H}$ NMR $(300$ $\left.\mathrm{MHz}, 25^{\circ} \mathrm{C}, \mathrm{C}_{6} \mathrm{D}_{6}\right), \delta$ (ppm): 6.23 (s, 3H, PhH TMB), 4.42 (t, $\left.4 \mathrm{H}, \mathrm{OCH}_{2} \mathrm{OX}\right), 3.34$ (s, $9 \mathrm{H}, \mathrm{CH}_{3}$ TMB), 2.11 (m, 2H, $\left.\mathrm{OCH}_{2} \mathrm{CH}_{2} \mathrm{OX}\right)$. 


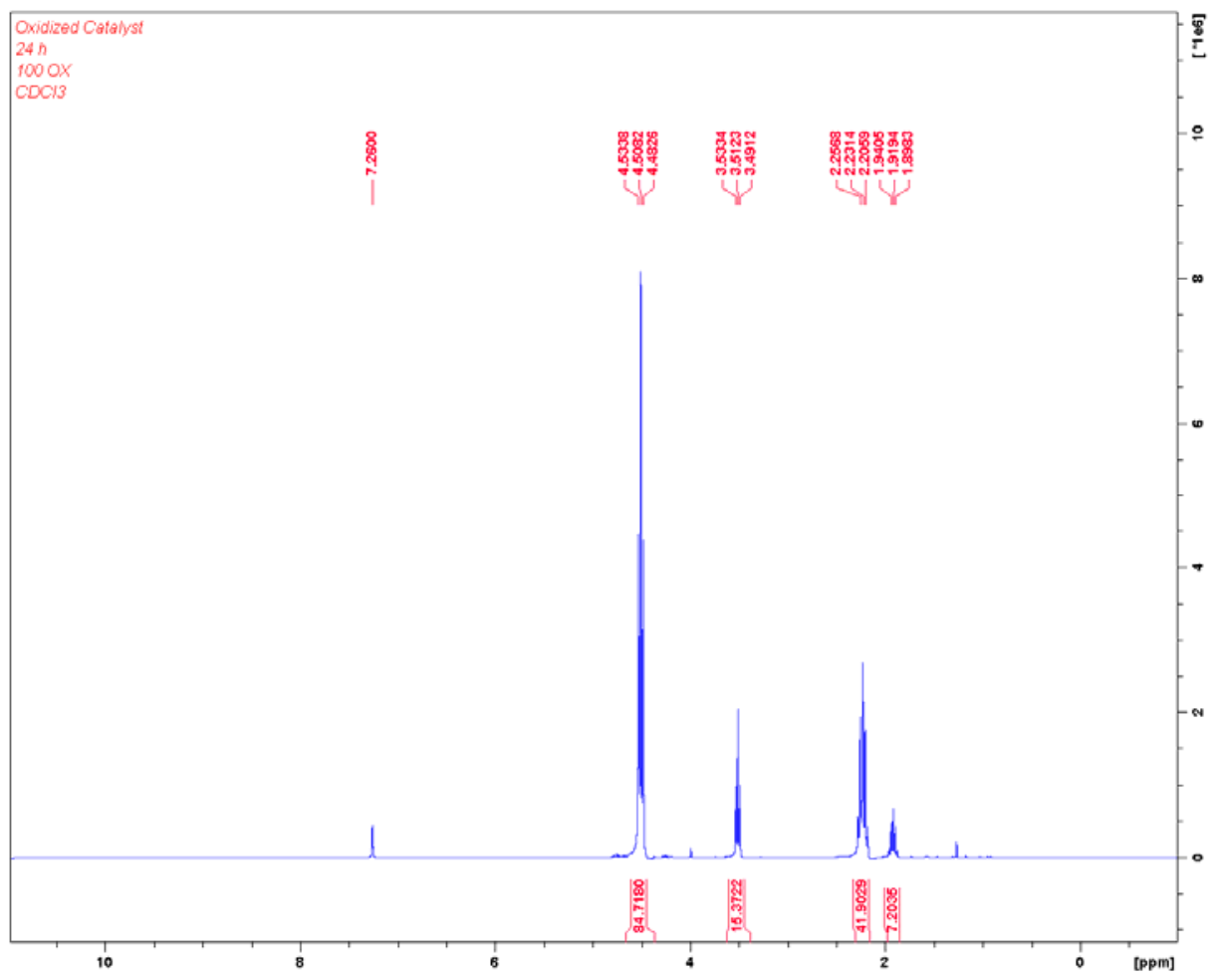

Figure S13. Table 1, entry 12. Polymerization of oxetane by [(salfan) $\left.\mathrm{Zr}\left(\mathrm{O}^{t} \mathrm{Bu}\right)_{2}\right]\left[\mathrm{BAr}{ }^{\mathrm{F}} 4\right] .{ }^{1} \mathrm{H}$ NMR ( $\left.300 \mathrm{MHz}, 25^{\circ} \mathrm{C}, \mathrm{CDCl}_{3}\right), \delta(\mathrm{ppm}): 4.51$ (t, $\left.4 \mathrm{H}, \mathrm{OCH}_{2} \mathrm{OX}\right), 3.51$ (t, $\left.4 \mathrm{H}, \mathrm{OCH}_{2} \mathrm{POX}\right), 2.23$ (m, $\left.2 \mathrm{H}, \mathrm{OCH}_{2} \mathrm{CH}_{2} \mathrm{OX}\right), 1.92\left(\mathrm{~m}, 2 \mathrm{H}, \mathrm{OCH}_{2} \mathrm{CH}_{2} \mathrm{OX}\right.$ ). 


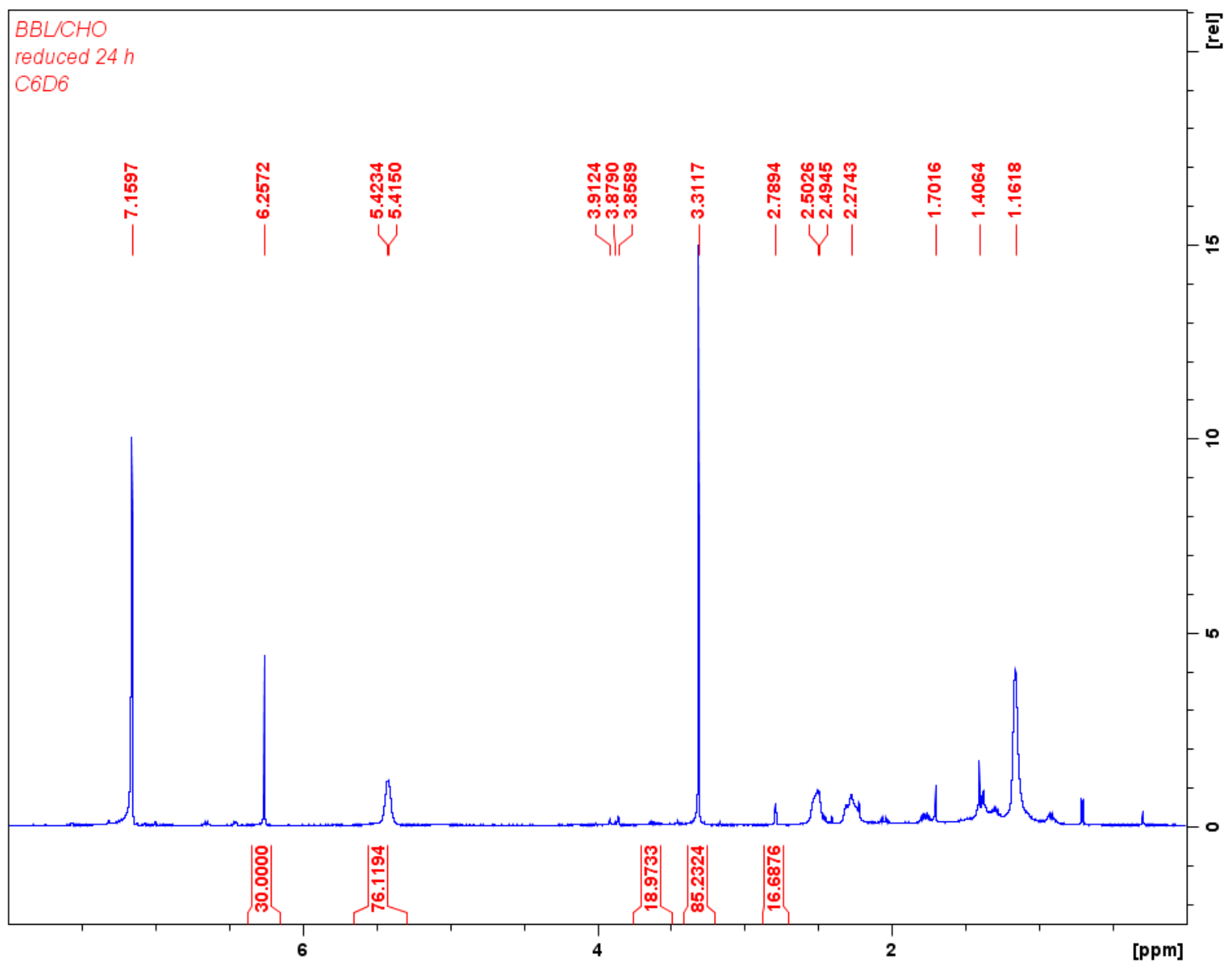

Figure S14. Table 2, entry 1. Polymerization of 100 equivalents of $\beta$-butyrolactone in the presence of 100 equivalents of cyclohexene oxide with catalyst in the reduced state. ${ }^{1} \mathrm{H}$ NMR $\left(300 \mathrm{MHz}, 25^{\circ} \mathrm{C}, \mathrm{C}_{6} \mathrm{D}_{6}\right), \delta(\mathrm{ppm}): 6.26$ (s, 3H, $\left.\mathrm{PhH} \mathrm{TMB}\right), 5.42$ (m, 1H, $\left.\mathrm{OCHCH}_{3} \mathrm{PHB}\right), 3.87$ $\left(\mathrm{m}, 1 \mathrm{H}, \mathrm{OCHCH}_{3}, \mathrm{BBL}\right), 3.62(\mathrm{~m}, 2 \mathrm{H}, \mathrm{COCH} \mathrm{PCHO}), 3.31$ (s, 9H, CH3 TMB), 2.79 (s, 2H, $\mathrm{COCH}_{2} \mathrm{CHO}$ ), 2.50-2.27 (m, 2H, $\left.\mathrm{COCH}_{2} \mathrm{PHB}\right), 1.70$ (m, 2H, $\left.\mathrm{COCHCH}_{2} \mathrm{PCHO}\right), 1.40(\mathrm{~m}, 2 \mathrm{H}$, $\left.\mathrm{COCHCH}_{2} \mathrm{CH}_{2} \mathrm{PCHO}\right), 1.31$ (m, 2H, $\left.\mathrm{COCHCH}_{2} \mathrm{CH}_{2} \mathrm{PCHO}\right), 1.16\left(\mathrm{t}, 3 \mathrm{H}, \mathrm{OCHCH}_{3} \mathrm{PHB}\right), 0.89$ ( $\mathrm{t}, 3 \mathrm{H}, \mathrm{OCHCH}_{3} \mathrm{BBL}$ ). 


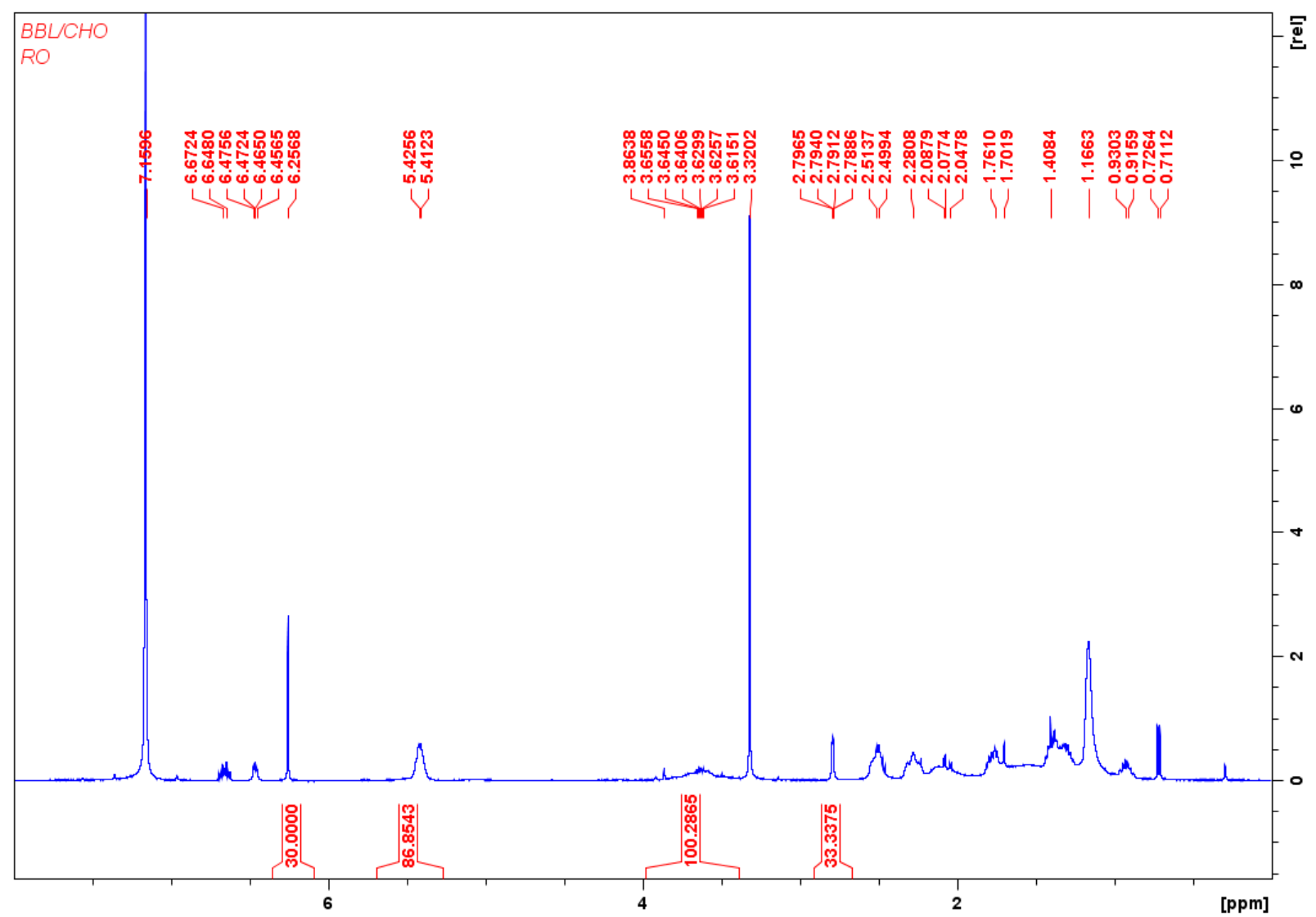

Figure S15. Table 2, entry 2. Polymerization of 100 equivalents of $\beta$-butyrolactone in the presence of 100 equivalents of cyclohexene oxide with catalyst in the reduced state and subsequently oxidized. ${ }^{1} \mathrm{H}$ NMR $\left(300 \mathrm{MHz}, 25{ }^{\circ} \mathrm{C}, \mathrm{C}_{6} \mathrm{D}_{6}\right), \delta(\mathrm{ppm}): 6.65$ (CFCH 1,2difluorobenzene), 6.47 (CFCHCH 1,2-difluorobenzene), 6.26 (s, 3H, $\mathrm{PhH} \mathrm{TMB}), 5.42$ (m, 1H, $\left.\mathrm{OCHCH}_{3} \mathrm{PHB}\right), 3.62$ (m, 2H, COCH PCHO), 3.32 (s, 9H, CH3 TMB), 2.79 (s, 2H, $\mathrm{COCH}_{2}$ $\mathrm{CHO}), 2.50-2.28\left(\mathrm{~m}, 2 \mathrm{H}, \mathrm{COCH}_{2} \mathrm{PHB}\right), 2.07$ (m, 2H, $\left.\mathrm{COCHCH}_{2} \mathrm{PCHO}\right), 1.76(\mathrm{~m}, 2 \mathrm{H}$, $\left.\mathrm{COCHCH}_{2} \mathrm{PCHO}\right), 1.41\left(\mathrm{~m}, 2 \mathrm{H}, \mathrm{COCHCH}_{2} \mathrm{CH}_{2} \mathrm{PCHO}\right), 1.17\left(\mathrm{~m}, 2 \mathrm{H}, \mathrm{OCHCH}_{3} \mathrm{PHB}\right), 0.93$ (m, $\left.2 \mathrm{H}, \mathrm{COCHCH}_{2} \mathrm{CH}_{2} \mathrm{CHO}\right), 0.71$ ( $\left.\mathrm{t}, 3 \mathrm{H}, \mathrm{OCHCH}_{3} \mathrm{BBL}\right)$. 


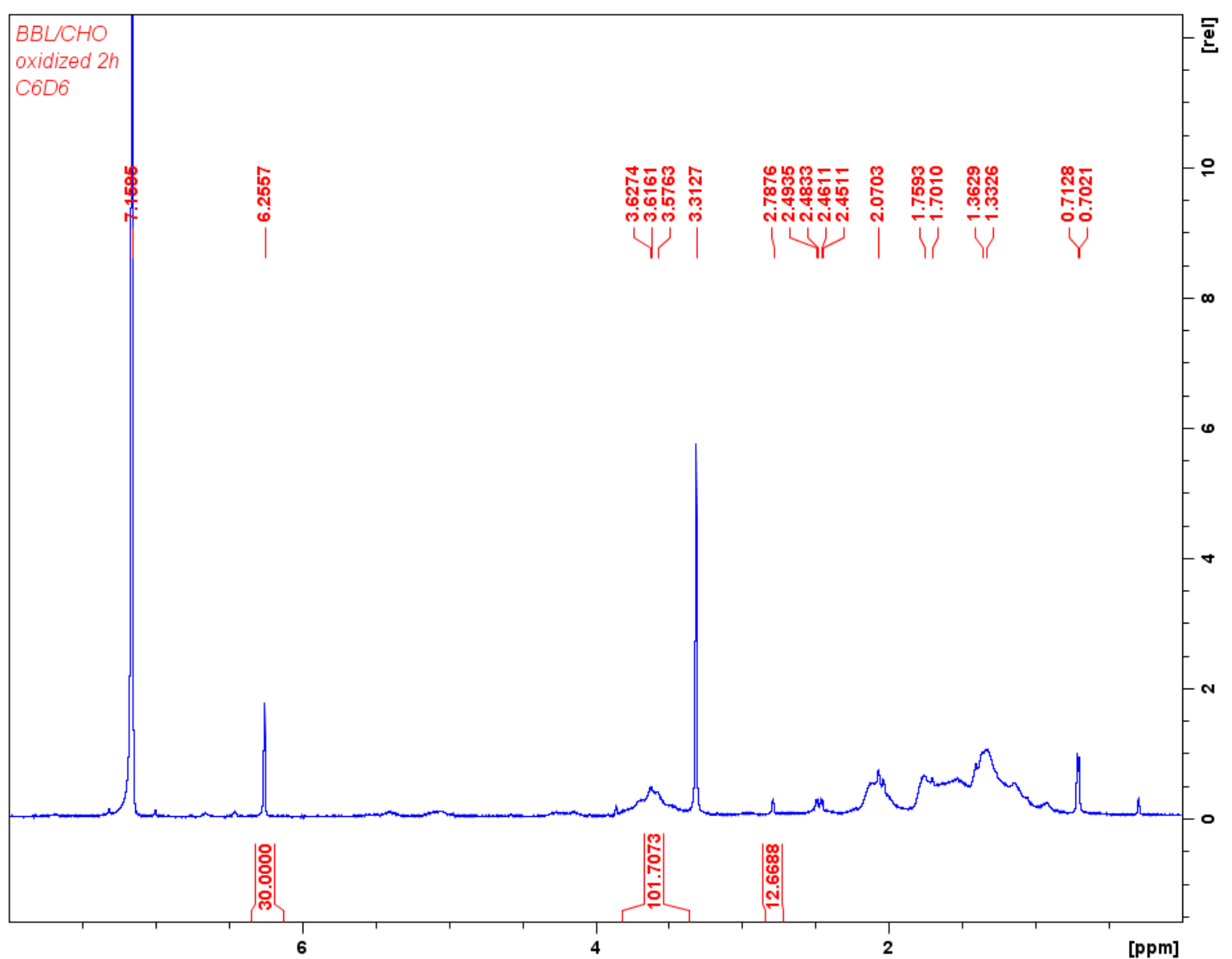

Figure S16. Table 2, entry 3. Polymerization of 100 equivalents of $\beta$-butyrolactone in the presence of 100 equivalents of cyclohexene oxide with catalyst in the oxidized state. ${ }^{1} \mathrm{H} \mathrm{NMR}$ (300 MHz, $\left.25^{\circ} \mathrm{C}, \mathrm{C}_{6} \mathrm{D}_{6}\right), \delta(\mathrm{ppm}): 6.26$ (s, 3H, PhH TMB), 3.62 (m, 2H, COCH PCHO), 3.31 (s, $\left.9 \mathrm{H}, \mathrm{CH}_{3} \mathrm{TMB}\right), 2.78$ (s, 2H, $\left.\mathrm{COCH}_{2} \mathrm{CHO}\right), 2.47$ (m, 2H, $\left.\mathrm{COCH}_{2} \mathrm{BBL}\right), 2.07$ (m, 2H, $\left.\mathrm{COCHCH}_{2} \mathrm{PCHO}\right), 1.74$ (m, 2H, $\left.\mathrm{COCHCH}_{2} \mathrm{PCHO}\right), 1.34$ (m, 2H, $\left.\mathrm{COCHCH}_{2} \mathrm{CH}_{2} \mathrm{PCHO}\right), 0.71$ (t, 3H, $\left.\mathrm{OCHCH}_{3} \mathrm{BBL}\right)$. 


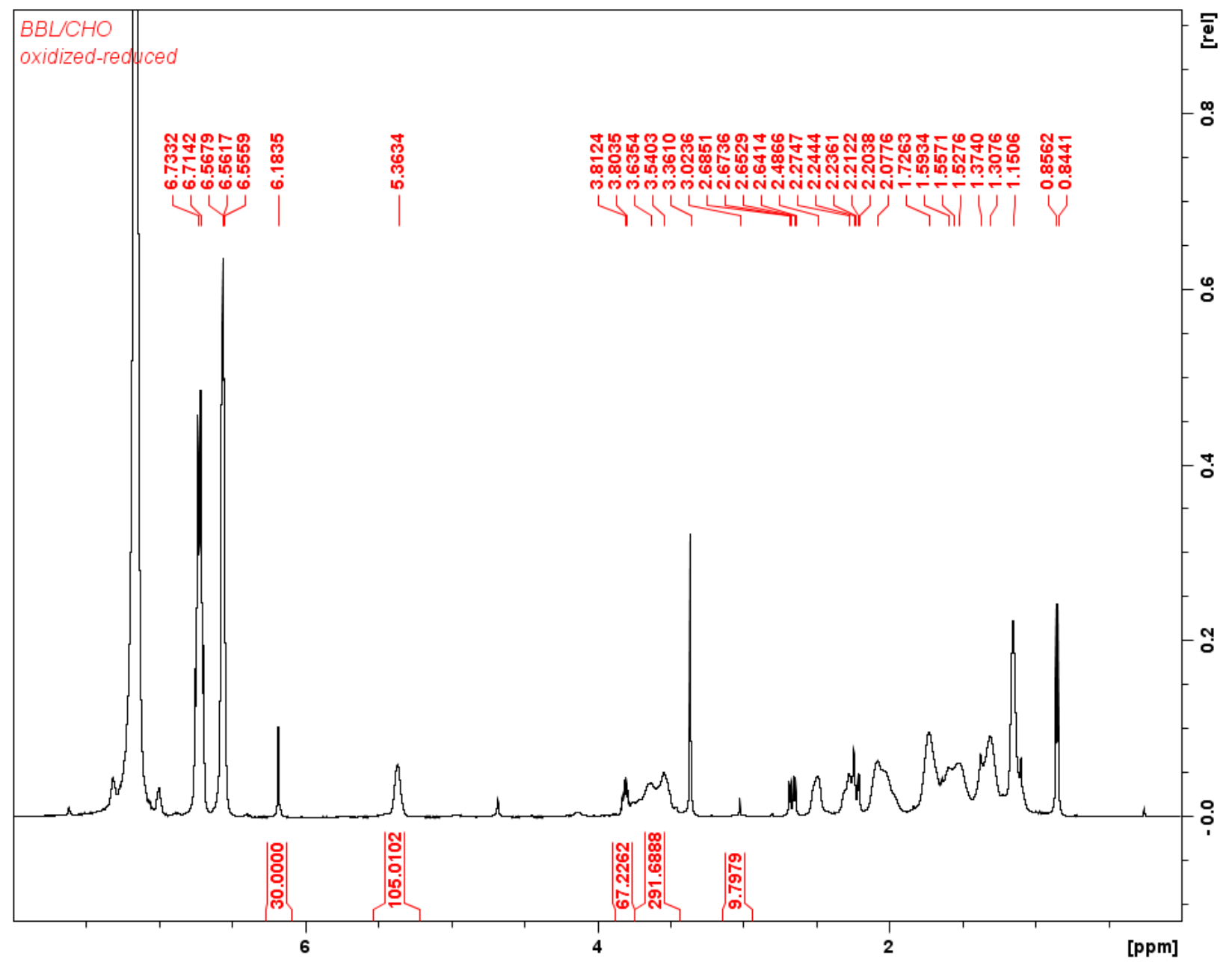

Figure S17. Table 2, entry 4. Polymerization of 100 equivalents of $\beta$-butyrolactone in the presence of 100 equivalents of propylene oxide with catalyst in the oxidized state and subsequently reduced. ${ }^{1} \mathrm{H}$ NMR $\left(300 \mathrm{MHz}, 25{ }^{\circ} \mathrm{C}, \mathrm{C}_{6} \mathrm{D}_{6}\right), \delta$ (ppm): 6.71 (CFCH 1,2difluorobenzene), 6.55 (CFCHCH 1,2-difluorobenzene), 6.18 (s, 3H, $\mathrm{PhH} \mathrm{TMB}), 5.36$ (m, 1H, $\left.\mathrm{OCHCH}_{3} \mathrm{PHB}\right), 3.81\left(\mathrm{~m}, 1 \mathrm{H}, \mathrm{OCHCH}_{3}, \mathrm{BBL}\right), 3.58(\mathrm{~m}, 2 \mathrm{H}, \mathrm{COCH} \mathrm{PCHO}), 3.36\left(\mathrm{~s}, 9 \mathrm{H}, \mathrm{CH}_{3}\right.$ TMB), 3.02 (s, 2H, $\left.\mathrm{COCH}_{2} \mathrm{CHO}\right), 2.67-2.24\left(\mathrm{~m}, 2 \mathrm{H}, \mathrm{COCH}_{2} \mathrm{BBL}\right), 2.48-2.27$ (m, 2H, $\mathrm{COCH}_{2}$ $\mathrm{PHB}), 2.07\left(\mathrm{~m}, 2 \mathrm{H}, \mathrm{COCHCH}_{2} \mathrm{PCHO}\right), 1.73\left(\mathrm{~m}, 2 \mathrm{H}, \mathrm{COCHCH}_{2} \mathrm{PCHO}\right), 1.37(\mathrm{~m}, 2 \mathrm{H}$, $\left.\mathrm{COCHCH}_{2} \mathrm{CH}_{2} \mathrm{PCHO}\right), 1.31\left(\mathrm{~m}, 2 \mathrm{H}, \mathrm{COCHCH}_{2} \mathrm{CH}_{2} \mathrm{PCHO}\right), 1.15\left(\mathrm{t}, 3 \mathrm{H}, \mathrm{OCHCH}_{3} \mathrm{PHB}\right), 0.85$ ( $\mathrm{t}, 3 \mathrm{H}, \mathrm{OCHCH}_{3} \mathrm{BBL}$ ). 


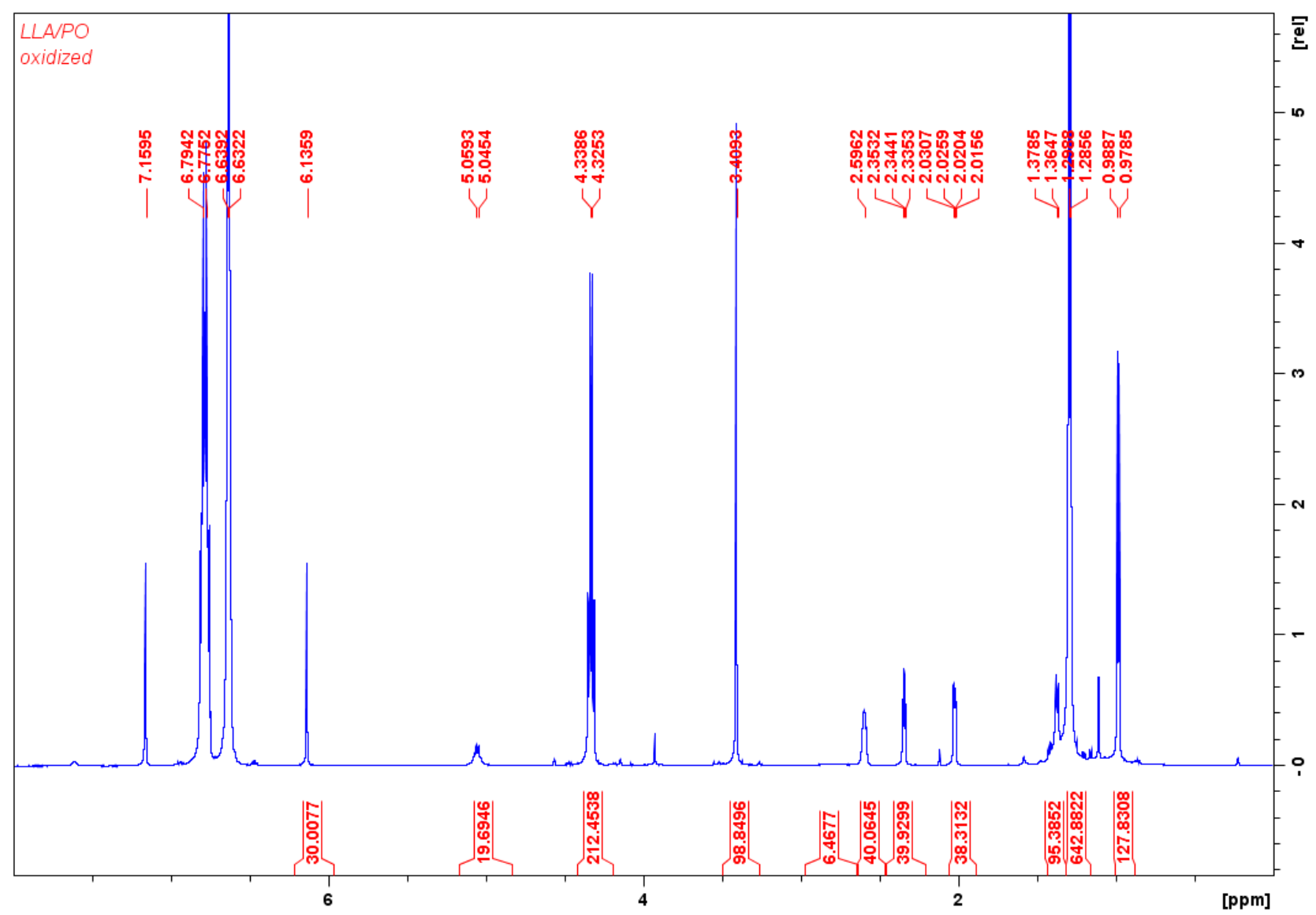

Figure S18. Table 2, entry 5. Polymerization of 100 equivalents of L-lactide in the presence of 100 equivalents of propylene oxide with catalyst in the oxidized state. ${ }^{1} \mathrm{H} \mathrm{NMR}(300 \mathrm{MHz}, 25$ ${ }^{\circ} \mathrm{C}, \mathrm{C}_{6} \mathrm{D}_{6}$ ), $\delta$ (ppm): 6.78 (CFCH 1,2-difluorobenzene), 6.63 (CFCHCH 1,2-difluorobenzene), 6.14 (s, 3H, PhH TMB), 5.04 (q, 2H, $\left.\mathrm{CHCH}_{3} \mathrm{PLA}\right), 4.33$ (q, 2H, $\left.\mathrm{CHCH}_{3} \mathrm{LLA}\right), 3.60$ (m, 1H, $\left.\mathrm{COCHCH}_{3} \mathrm{PPO}\right), 3.40$ (s, 9H, $\left.\mathrm{CH}_{3} \mathrm{TMB}\right), 2.60\left(\mathrm{~m}, 1 \mathrm{H}, \mathrm{COCHCH}_{3} \mathrm{PO}\right), 2.34\left(\mathrm{~m}, 1 \mathrm{H}, \mathrm{COCH}_{2}\right.$ PO), 2.02 (m, 1H, $\mathrm{COCH}_{2} \mathrm{PO}$ ), 1.36 (d, 3H, $\left.\mathrm{CHCH}_{3} \mathrm{PLA}\right), 1.29$ (d, 6H, $\mathrm{CHCH}_{3} \mathrm{LA}$ ), 0.98 (d, $\left.3 \mathrm{H}, \mathrm{COCHCH}_{3} \mathrm{PO}\right)$. 


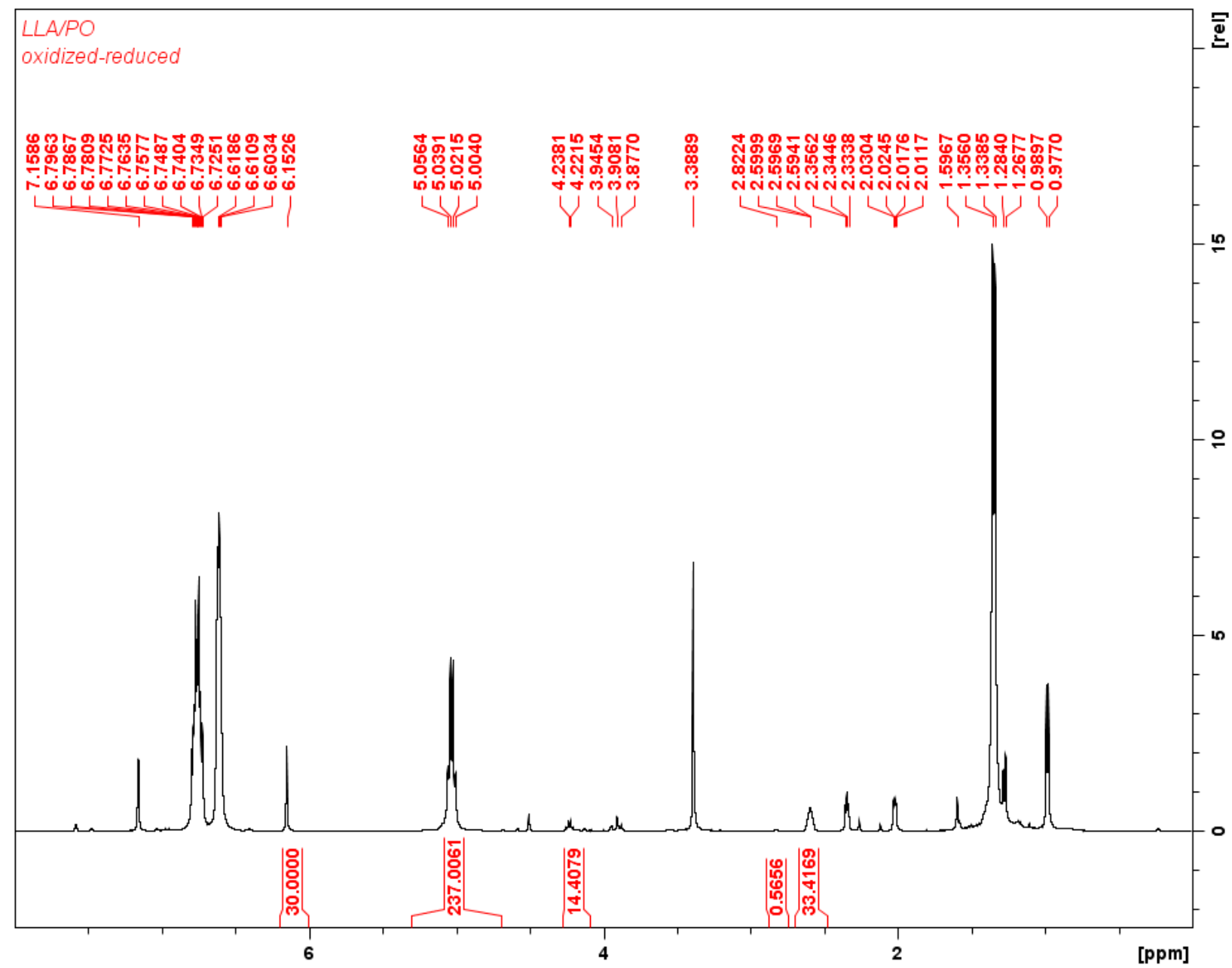

Figure S19. Table 2, entry 6. Polymerization of 100 equivalents of L-lactide in the presence of 100 equivalents of propylene oxide with catalyst in the oxidized state and subsequently reduced. ${ }^{1} \mathrm{H}$ NMR (300 MHz, $25{ }^{\circ} \mathrm{C}, \mathrm{C}_{6} \mathrm{D}_{6}$ ), $\delta$ (ppm): 6.76 (CFCH 1,2-difluorobenzene), 6.60 (CFCHCH 1,2-difluorobenzene), 6.15 (s, 3H, $\mathrm{PhH} \mathrm{TMB),} 5.03$ (q, 2H, $\mathrm{CHCH}_{3} \mathrm{PLA}$ ), 4.23 (q, 2H, $\mathrm{CHCH}_{3}$ LLA), 3.39 (s, 9H, $\left.\mathrm{CH}_{3} \mathrm{TMB}\right), 2.82$ (m, 1H, $\left.\mathrm{COCHCH}_{3} \mathrm{PPO}\right), 2.60$ (m, 1H, COCHCH3 PO), $2.34\left(\mathrm{~m}, 1 \mathrm{H}, \mathrm{COCH}_{2} \mathrm{PO}\right), 2.02\left(\mathrm{~m}, 1 \mathrm{H}, \mathrm{COCH}_{2} \mathrm{PO}\right), 1.60$ (d, 3H, COCHCH $\left.3 \mathrm{PPO}\right), 1.34$ (d, 6H, $\left.\mathrm{CHCH}_{3} \mathrm{PLA}\right), 1.27$ (d, 6H, $\mathrm{CHCH}_{3}$ L-lactide), 0.98 (d, 3H, $\mathrm{COCHCH}_{3} \mathrm{PO}$ ). 


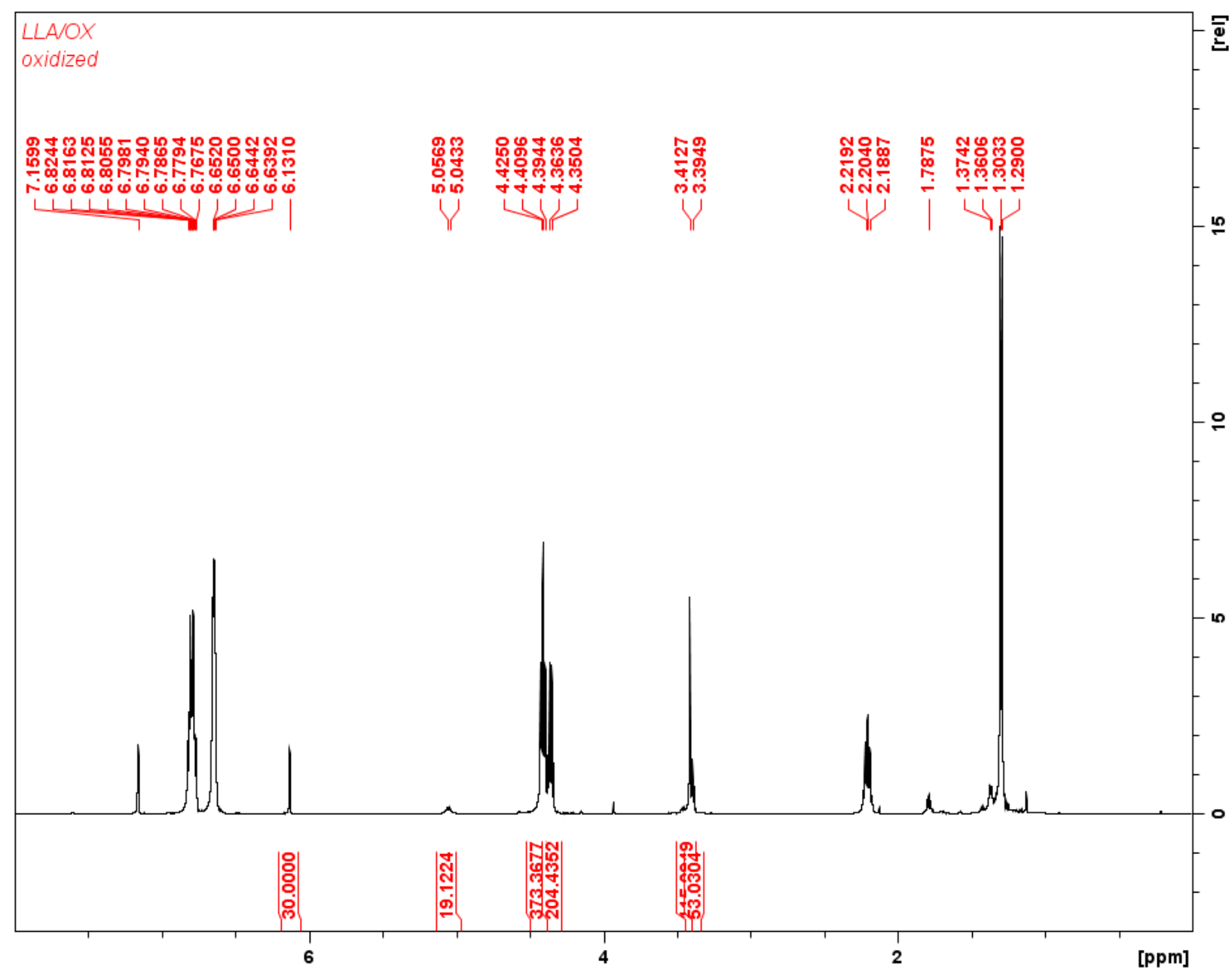

Figure S20. Table 2, entry 7. Polymerization of 100 equivalents of L-lactide in the presence of 100 equivalents of oxetane with catalyst in the oxidized state. ${ }^{1} \mathrm{H}$ NMR $\left(300 \mathrm{MHz}, 25^{\circ} \mathrm{C}, \mathrm{C}_{6} \mathrm{D}_{6}\right)$, $\delta$ (ppm): 6.77 (CFCH 1,2-difluorobenzene), 6.65 (CFCHCH 1,2-difluorobenzene), 6.13 (s, 3H, $\mathrm{PhH} \mathrm{TMB}$ ), 5.04 (q, 2H, $\left.\mathrm{CHCH}_{3} \mathrm{PLA}\right), 4.41$ (t, 4H, $\left.\mathrm{OCH}_{2} \mathrm{OX}\right), 4.36$ (q, 2H, $\left.\mathrm{CHCH}_{3} \mathrm{LA}\right), 3.40$ $\left(\mathrm{s}, 9 \mathrm{H}, \mathrm{CH}_{3} \mathrm{TMB}\right), 3.39$ (t, 4H, OCH $\mathrm{POX}_{2}, 2.20\left(\mathrm{~m}, 2 \mathrm{H}, \mathrm{OCH}_{2} \mathrm{CH}_{2} \mathrm{OX}\right), 1.79$ (m, 2H, $\mathrm{OCH}_{2} \mathrm{CH}_{2} \mathrm{POX}$ ), 1.37 (d, 6H, $\left.\mathrm{CHCH}_{3} \mathrm{PLA}\right), 1.30$ (d, 6H, $\left.\mathrm{CHCH}_{3} \mathrm{LA}\right)$. 


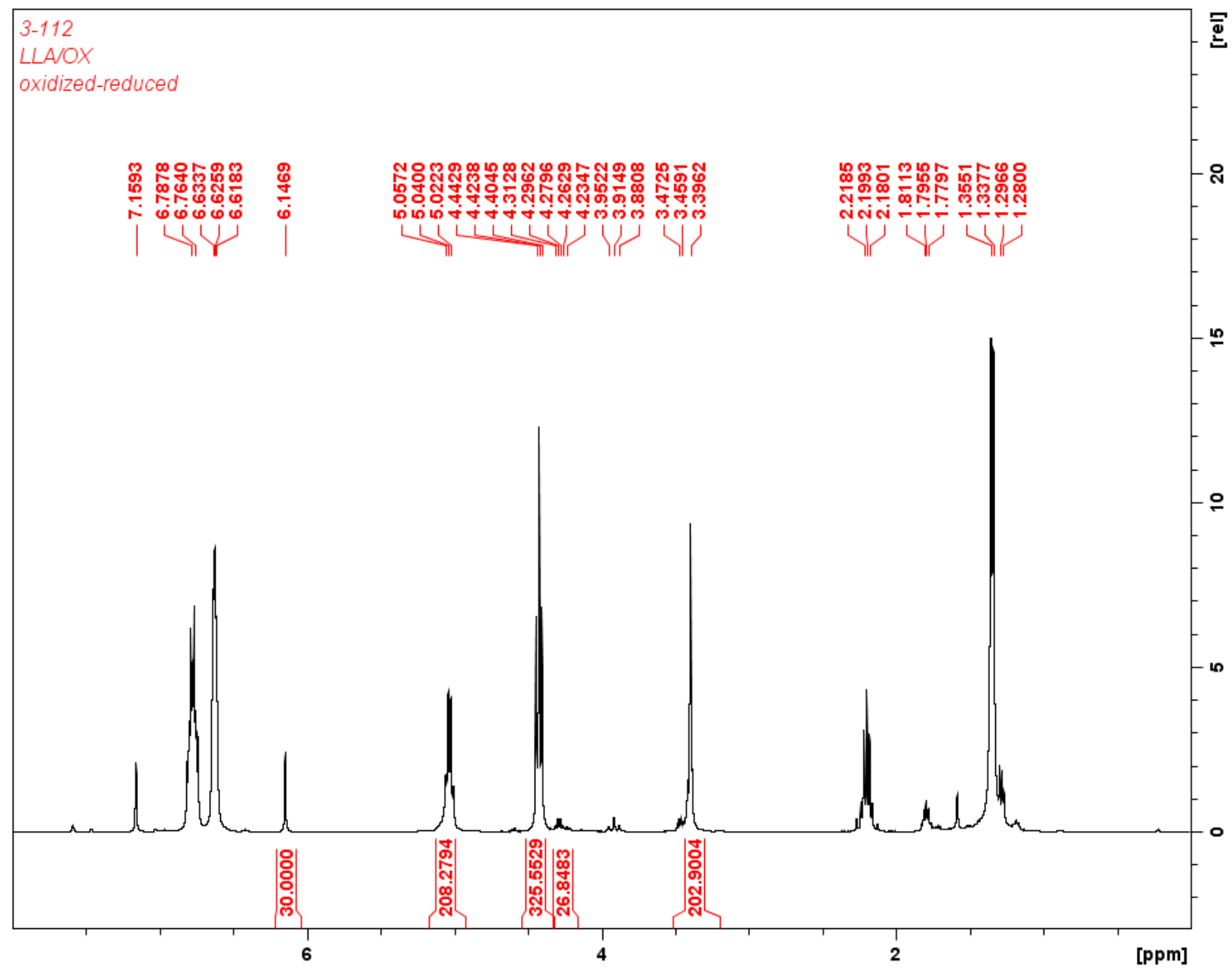

Figure S21. Table 2, entry 8. Polymerization of 100 equivalents of L-lactide in the presence of 100 equivalents of oxetane with catalyst in the oxidized state and subsequently reduced. ${ }^{1} \mathrm{H}$ NMR (300 MHz, $\left.25^{\circ} \mathrm{C}, \mathrm{C}_{6} \mathrm{D}_{6}\right), \delta$ (ppm): 6.77 (CFCH 1,2-difluorobenzene), 6.62 (CFCHCH 1,2difluorobenzene), 6.15 (s, 3H, $\mathrm{PhH} \mathrm{TMB}$ ), 5.04 (q, 2H, $\left.\mathrm{CHCH}_{3} \mathrm{PLA}\right), 4.42$ (t, 4H, OCH $\mathrm{OX}_{2}$ ), 4.27 (q, 2H, $\left.\mathrm{CHCH}_{3} \mathrm{LA}\right), 3.47$ (t, 4H, OCH $\left.2 \mathrm{POX}\right), 3.39$ (s, 9H, $\left.\mathrm{CH}_{3} \mathrm{TMB}\right), 2.20$ (m, 2H, $\mathrm{OCH}_{2} \mathrm{CH}_{2} \mathrm{OX}$ ), 1.79 (m, 2H, $\mathrm{OCH}_{2} \mathrm{CH}_{2} \mathrm{OX}$ ), 1.34 (d, 6H, $\left.\mathrm{CHCH}_{3} \mathrm{PLA}\right), 1.29$ (d, 6H, $\mathrm{CHCH}_{3}$ LA). 


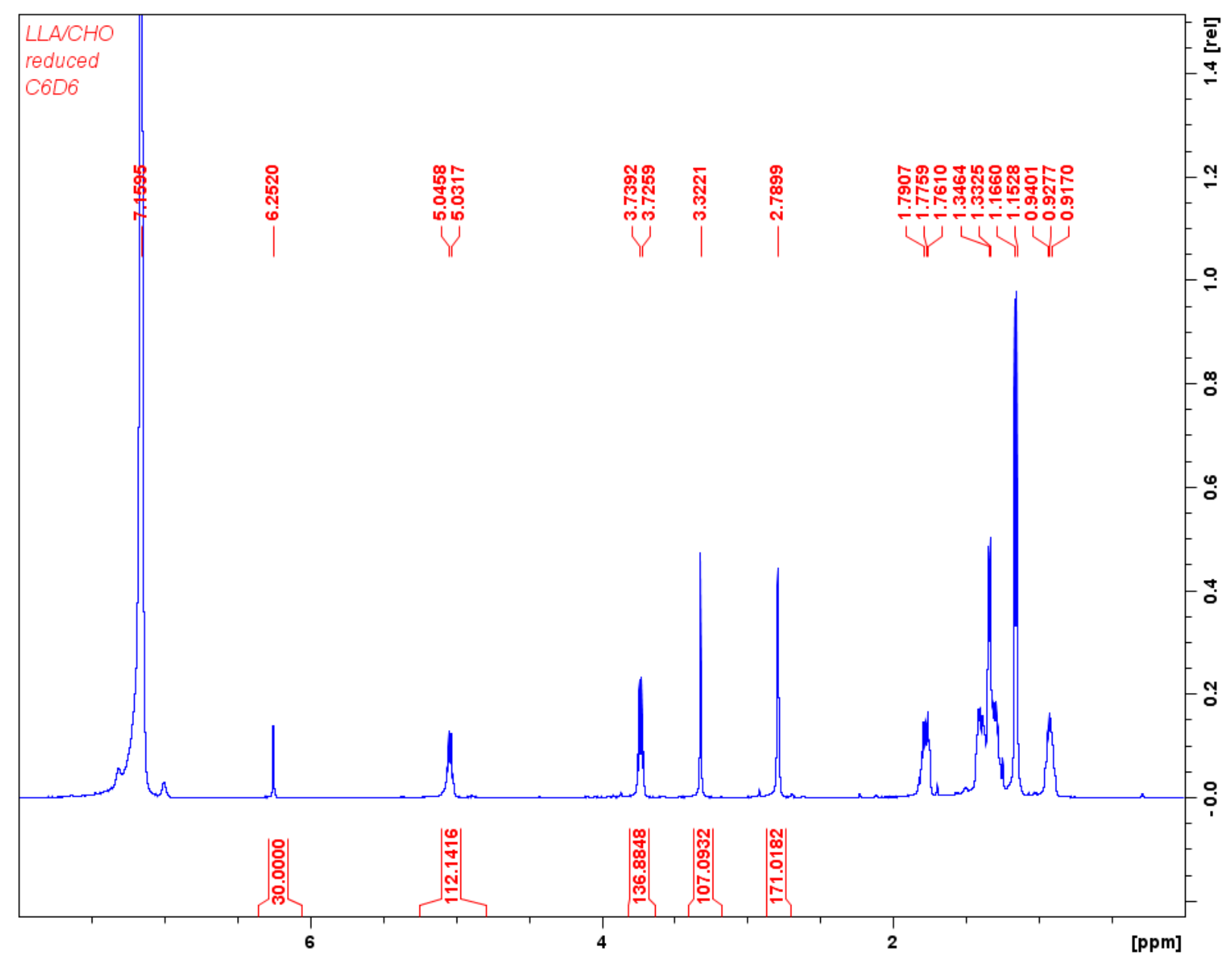

Figure S22. Table 2, entry 9. Polymerization of 100 equivalents of L-lactide in the presence of 100 equivalents of cyclohexene oxide with catalyst in the reduced state. ${ }^{1} \mathrm{H}$ NMR $(300 \mathrm{MHz}, 25$ ${ }^{\circ} \mathrm{C}, \mathrm{C}_{6} \mathrm{D}_{6}$ ), $\delta$ (ppm): 6.25 (s, 3H, PhH TMB), 5.04 (q, 2H, $\left.\mathrm{CHCH}_{3} \mathrm{PLA}\right), 3.73$ (q, 2H, $\mathrm{CHCH}_{3}$ $\mathrm{LA}), 3.32$ (s, 9H, $\left.\mathrm{CH}_{3} \mathrm{TMB}\right), 2.79$ (s, 2H, $\left.\mathrm{COCH}_{2} \mathrm{CHO}\right), 1.77$ (m, 2H, $\left.\mathrm{COCHCH}_{2} \mathrm{CHO}\right), 1.39$ $\left(\mathrm{m}, 2 \mathrm{H}, \mathrm{COCHCH}_{2} \mathrm{CHO}\right), 1.33$ (d, 6H, $\left.\mathrm{CHCH}_{3} \mathrm{PLA}\right), 1.29\left(\mathrm{~m}, 2 \mathrm{H}, \mathrm{COCHCH}_{2} \mathrm{CH}_{2} \mathrm{CHO}\right), 1.15$ (d, $\left.6 \mathrm{H}, \mathrm{CHCH}_{3} \mathrm{LA}\right), 0.93$ (m, 2H, $\left.\mathrm{COCHCH}_{2} \mathrm{CH}_{2} \mathrm{CHO}\right)$. 


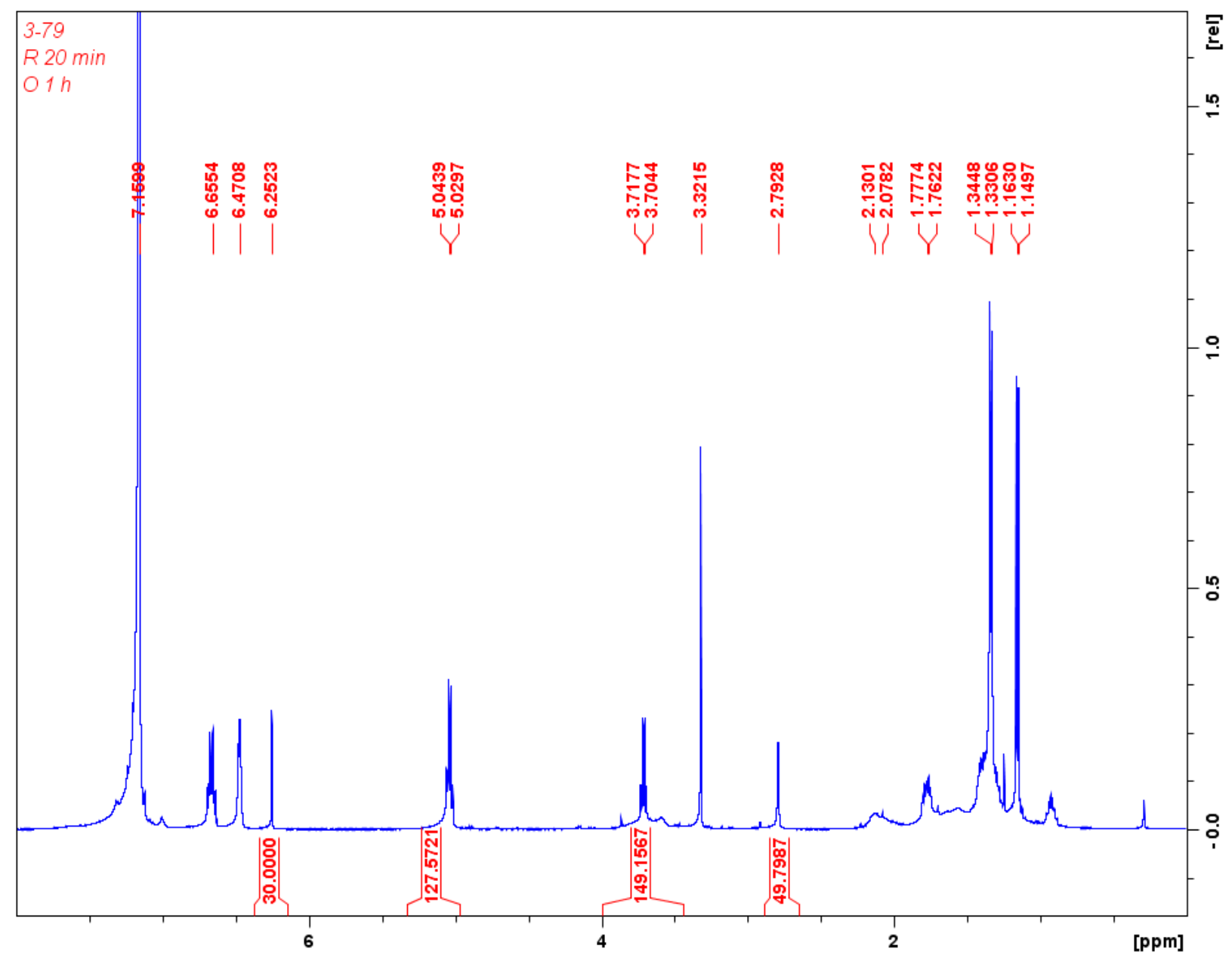

Figure S23. Table 2, entry 10. Polymerization of 100 equivalents of L-lactide in the presence of 100 equivalents of cyclohexene oxide with catalyst in the reduced state and subsequently oxidized. ${ }^{1} \mathrm{H}$ NMR $\left(300 \mathrm{MHz}, 25{ }^{\circ} \mathrm{C}, \mathrm{C}_{6} \mathrm{D}_{6}\right), \delta$ (ppm): 6.65 (CFCH 1,2-difluorobenzene), 6.47 (CFCHCH 1,2-difluorobenzene), 6.25 (s, 3H, $\mathrm{PhH} \mathrm{TMB),} 5.04$ (q, 2H, $\mathrm{CHCH}_{3}$ PLA), 3.71 (q, $\left.2 \mathrm{H}, \mathrm{CHCH}_{3} \mathrm{LLA}\right), 3.62$ (m, 2H, COCH PCHO), 3.32 (s, 9H, $\mathrm{CH}_{3} \mathrm{TMB}$ ), 2.79 (s, 2H, $\mathrm{COCH}_{2}$ $\mathrm{CHO}), 2.07$ (m, 2H, COCHCH$\left.{ }_{2} \mathrm{PCHO}\right), 1.77$ (m, 2H, $\left.\mathrm{COCHCH}_{2} \mathrm{CHO}\right), 1.33$ (d, 6H, $\mathrm{CHCH}_{3}$ PLA), 1.15 (d, 6H, $\left.\mathrm{CHCH}_{3} \mathrm{LLA}\right)$. 


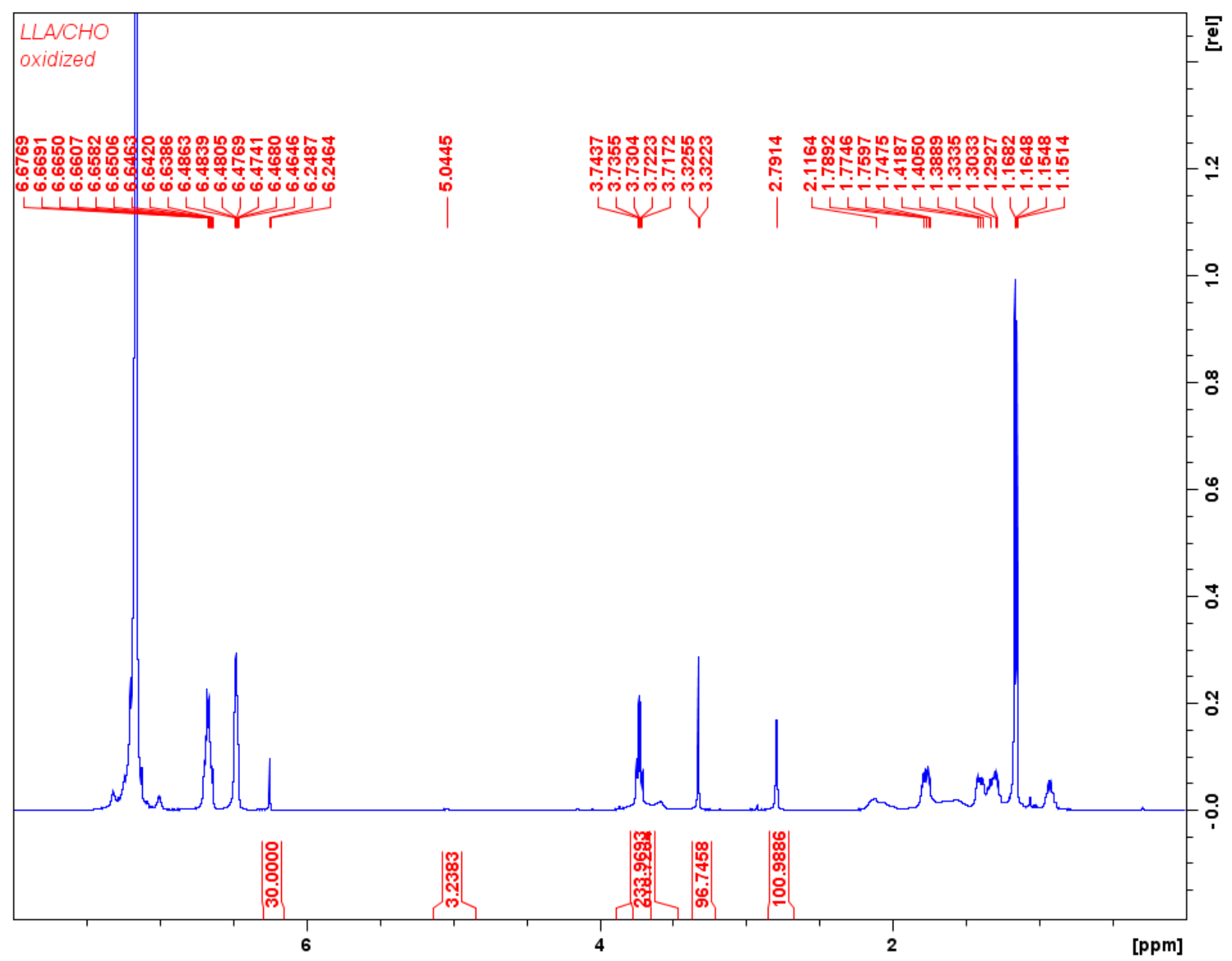

Figure S24. Table 2, entry 11. Polymerization of 100 equivalents of L-lactide in the presence of 100 equivalents of cyclohexene oxide with catalyst in the oxidized state. ${ }^{1} \mathrm{H}$ NMR $(300 \mathrm{MHz}, 25$ $\left.{ }^{\circ} \mathrm{C}, \mathrm{C}_{6} \mathrm{D}_{6}\right), \delta$ (ppm): 6.65 (CFCH 1,2-difluorobenzene), 6.47 (CFCHCH 1,2-difluorobenzene), 6.25 (s, 3H, $\mathrm{PhH}$ TMB), 5.04 (q, 2H, $\mathrm{CHCH}_{3} \mathrm{PLA}$ ), 3.73 (q, 2H, $\left.\mathrm{CHCH}_{3} \mathrm{LLA}\right), 3.62$ (m, 2H, $\mathrm{COCH} \mathrm{PCHO}$ ), 3.32 (s, 9H, $\left.\mathrm{CH}_{3} \mathrm{TMB}\right), 2.79$ (s, 2H, $\left.\mathrm{COCH}_{2} \mathrm{CHO}\right), 2.11$ (m, 2H, $\mathrm{COCHCH}_{2}$ $\mathrm{PCHO}), 1.77\left(\mathrm{~m}, 2 \mathrm{H}, \mathrm{COCHCH}_{2} \mathrm{CHO}\right), 1.39\left(\mathrm{~m}, 2 \mathrm{H}, \mathrm{COCHCH}_{2} \mathrm{CHO}\right), 1.31(\mathrm{~m}, 2 \mathrm{H}$, $\mathrm{COCHCH}_{2} \mathrm{CH}_{2} \mathrm{PCHO}$ ), 1.29 (m, 2H, $\left.\mathrm{COCHCH}_{2} \mathrm{CH}_{2} \mathrm{CHO}\right), 1.15$ (d, 6H, $\left.\mathrm{CHCH}_{3} \mathrm{LLA}\right)$. 


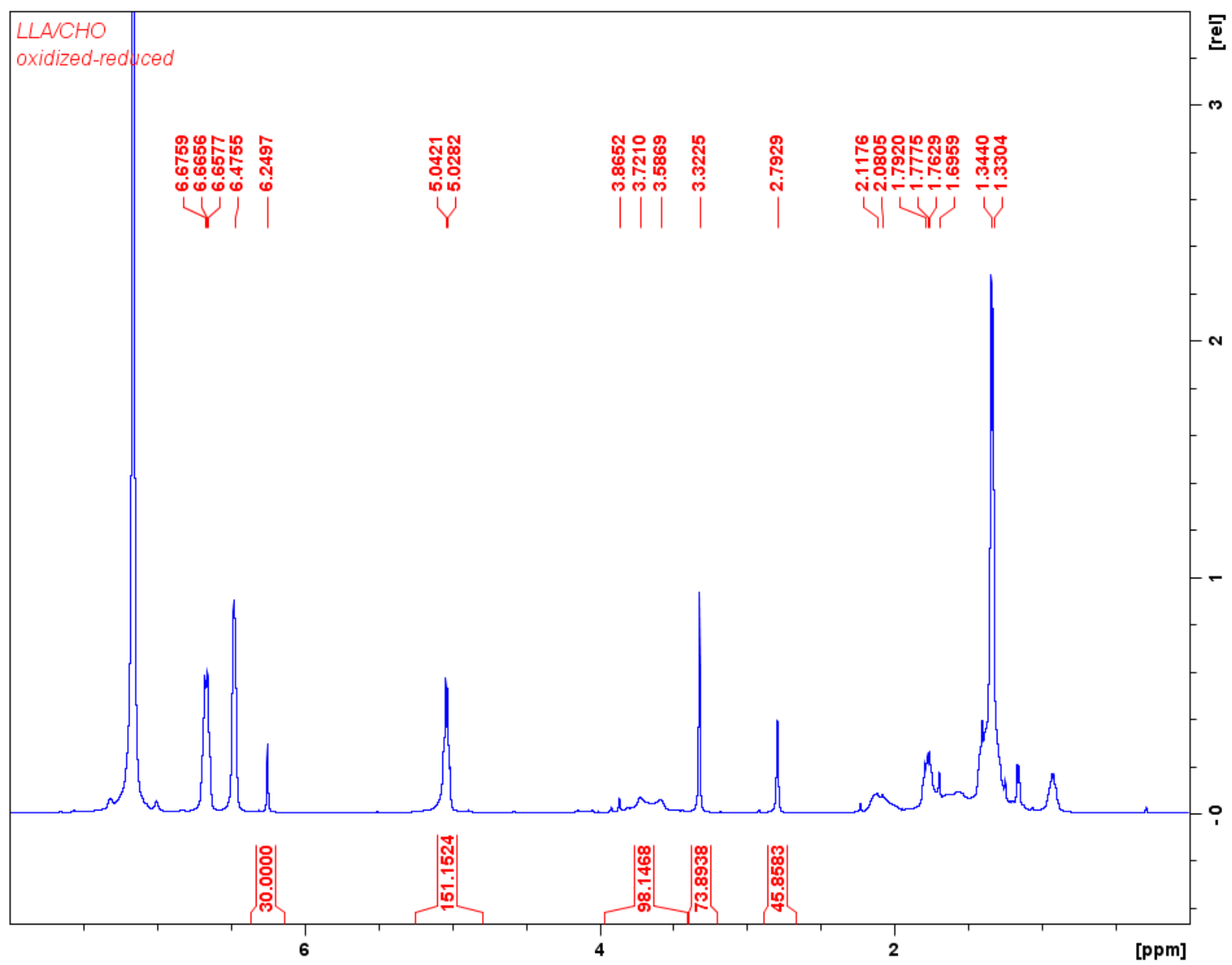

Figure S25. Table 2, entry 12. Polymerization of 100 equivalents of L-lactide in the presence of 100 equivalents of cyclohexene oxide with catalyst in the oxidized state and subsequently reduced. ${ }^{1} \mathrm{H}$ NMR $\left(300 \mathrm{MHz}, 25{ }^{\circ} \mathrm{C}, \mathrm{C}_{6} \mathrm{D}_{6}\right), \delta(\mathrm{ppm}): 6.66$ (CFCH 1,2-difluorobenzene), 6.48 (CFCHCH 1,2-difluorobenzene), 6.25 (s, 3H, $\mathrm{PhH} \mathrm{TMB),} 5.03$ (q, 2H, $\left.\mathrm{CHCH}_{3} \mathrm{PLA}\right), 3.65$ (m, 2H, COCH PCHO), 3.32 (s, 9H, $\left.\mathrm{CH}_{3} \mathrm{TMB}\right), 2.79$ (s, 2H, $\left.\mathrm{COCH}_{2} \mathrm{CHO}\right), 2.08$ (m, 2H, $\left.\mathrm{COCHCH}_{2} \mathrm{PCHO}\right), 1.77\left(\mathrm{~m}, 2 \mathrm{H}, \mathrm{COCHCH}_{2} \mathrm{CHO}\right), 1.70\left(\mathrm{~m}, 2 \mathrm{H}, \mathrm{COCHCH}_{2} \mathrm{CHO}\right), 1.33$ (d, 6H, $\left.\mathrm{CHCH}_{3} \mathrm{PLA}\right), 1.31$ (m, 2H, $\left.\mathrm{COCHCH}_{2} \mathrm{CH}_{2} \mathrm{PCHO}\right)$. 


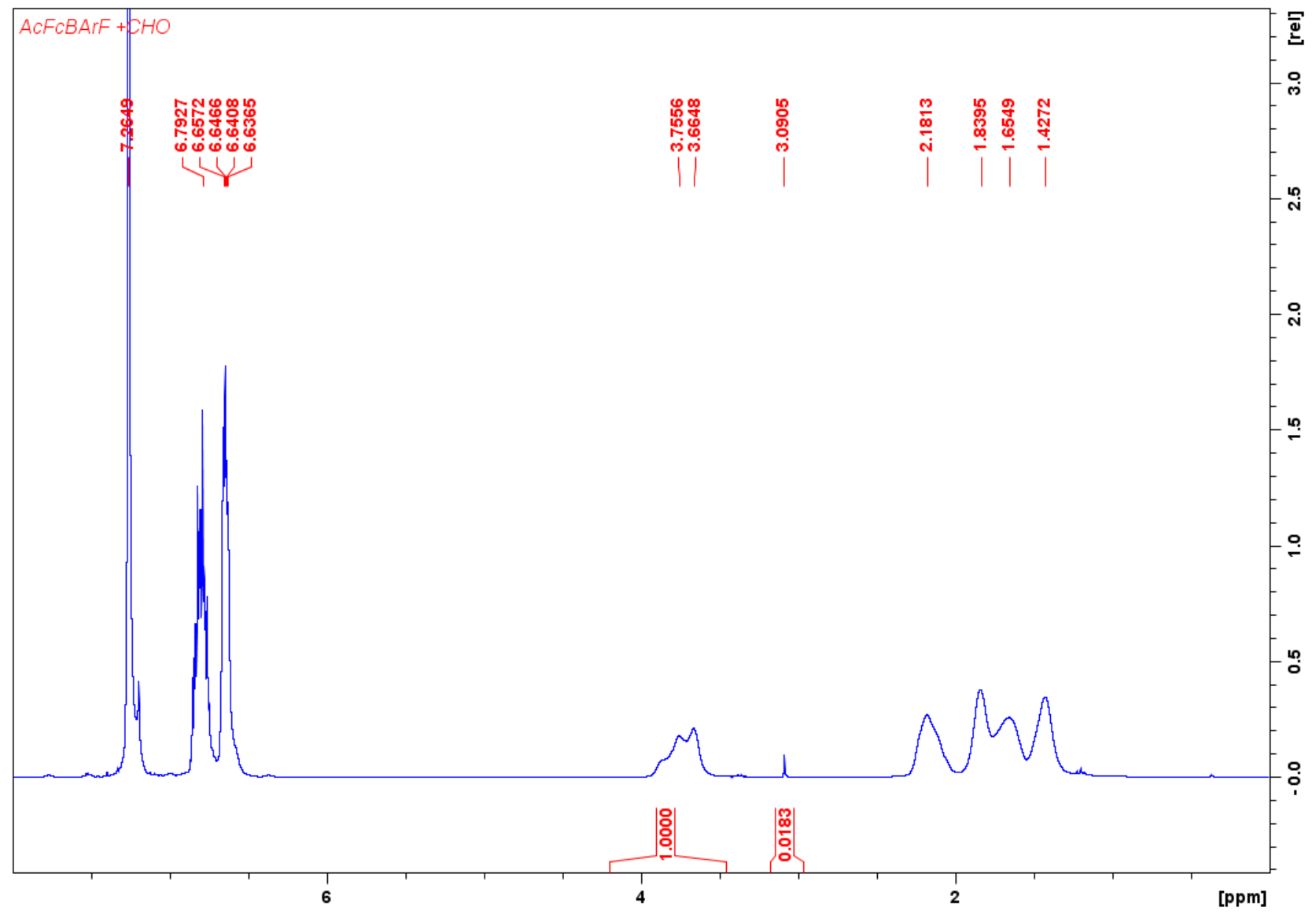

Figure S26. Table 3, entry 1. Polymerization of 100 equivalents of cyclohexene oxide by ${ }^{\mathrm{Ac}} \mathrm{FcBAr}^{\mathrm{F}} .{ }^{1} \mathrm{H}$ NMR $\left(300 \mathrm{MHz}, 25^{\circ} \mathrm{C}, \mathrm{CDCl}_{3}\right), \delta(\mathrm{ppm}): 6.79$ (CFCH 1,2-difluorobenzene), 6.65 (CFCHCH 1,2-difluorobenzene), 3.70 (m, 2H, COCH PCHO), 3.09 (s, 2H, $\mathrm{COCH}_{2} \mathrm{CHO}$ ), 2.18 (m, 2H, $\left.\mathrm{COCHCH}_{2} \mathrm{PCHO}\right), 1.84\left(\mathrm{~m}, 2 \mathrm{H}, \mathrm{COCHCH}_{2} \mathrm{PCHO}\right), 1.65\left(\mathrm{~m}, 2 \mathrm{H}, \mathrm{COCHCH}_{2} \mathrm{CH}_{2}\right.$ $\mathrm{PCHO}), 1.43$ (m, 2H, COCHCH $\left.\mathrm{CH}_{2} \mathrm{PCHO}\right)$. 


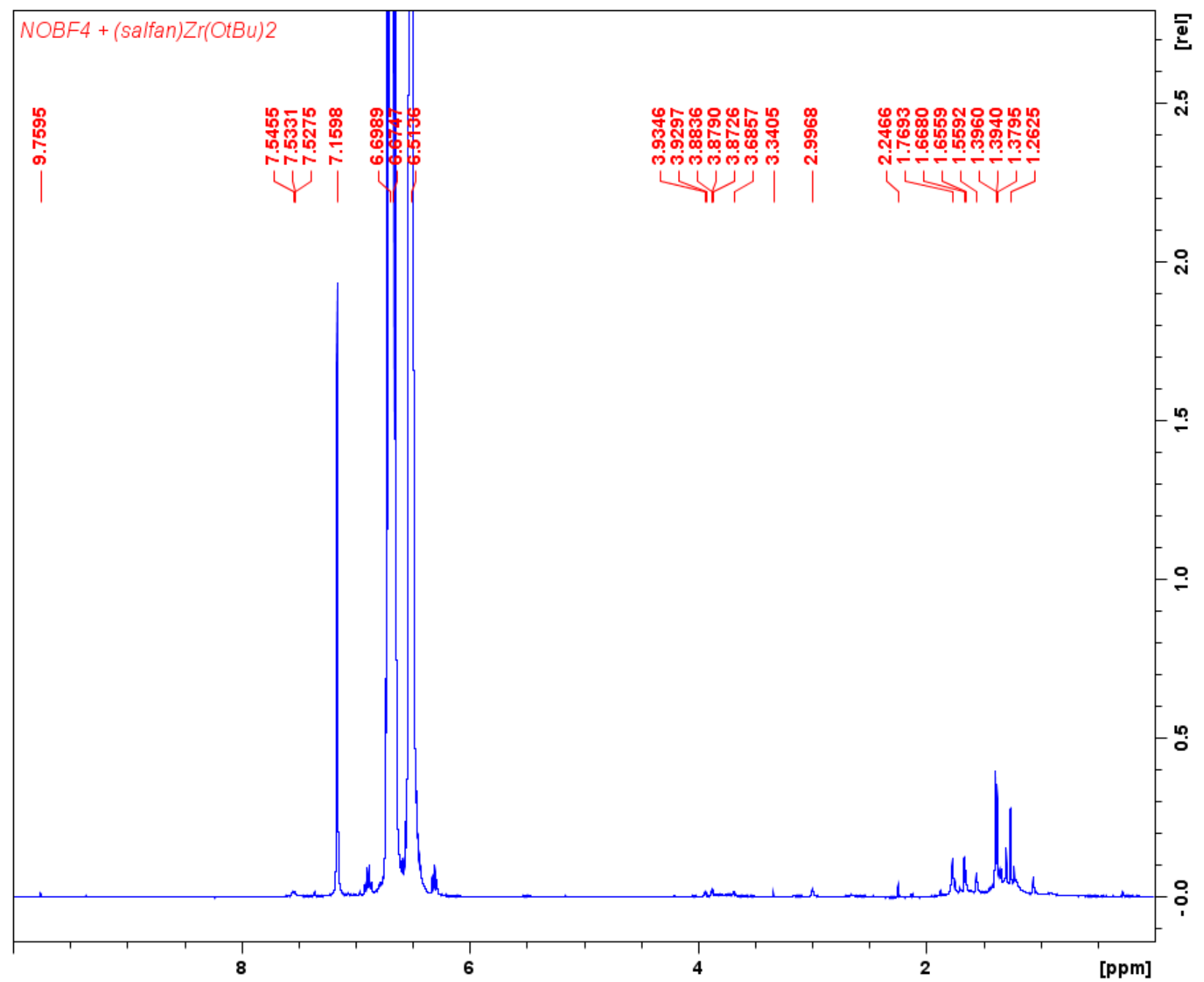

Figure S27. Table 3, entry 2. Oxidation of (salfan) $\mathrm{Zr}\left(\mathrm{O}^{t} \mathrm{Bu}\right)_{2}$ by $\mathrm{NOBF}_{4}{ }^{1} \mathrm{H}$ NMR $(300 \mathrm{MHz}, 25$ $\left.{ }^{\circ} \mathrm{C}, \mathrm{C}_{6} \mathrm{D}_{6}\right), \delta(\mathrm{ppm}): 9.76$ (s, 2H, OH), 7.53 (m, 2H, PhH), 6.68 (CFCH 1,2-difluorobenzene), 6.51 (CFCHCH 1,2-difluorobenzene), $3.93(\mathrm{~m}, 2 \mathrm{H}, \mathrm{CpH}), 3.88(\mathrm{~m}, 2 \mathrm{H}, \mathrm{CpH}), 3.68(\mathrm{~m}, 4 \mathrm{H}$, $\mathrm{CpH}), 3.34$ (s, 4H, NCH 2$\left.), 3.00\left(\mathrm{~s}, 6 \mathrm{H}, \mathrm{NCH}_{3}\right), 2.25\left(\mathrm{~s}, 6 \mathrm{H}, \mathrm{NCH}_{3}\right), 1.77\left(\mathrm{~s}, 18 \mathrm{H}, \mathrm{C}_{2} \mathrm{CH}_{3}\right)_{3}\right), 1.66$ $\left(\mathrm{s}, 18 \mathrm{H}, \mathrm{C}\left(\mathrm{CH}_{3}\right)_{3}\right), 1.56\left(\mathrm{~s}, 18 \mathrm{H}, \mathrm{C}\left(\mathrm{CH}_{3}\right)_{3}\right), 1.39\left(\mathrm{~s}, 18 \mathrm{H}, \mathrm{C}\left(\mathrm{CH}_{3}\right)_{3}\right), 1.38\left(\mathrm{~s}, 18 \mathrm{H}, \mathrm{C}\left(\mathrm{CH}_{3}\right)_{3}\right), 1.26$ $\left(\mathrm{s}, 18 \mathrm{H}, \mathrm{C}\left(\mathrm{CH}_{3}\right)_{3}\right)$. 


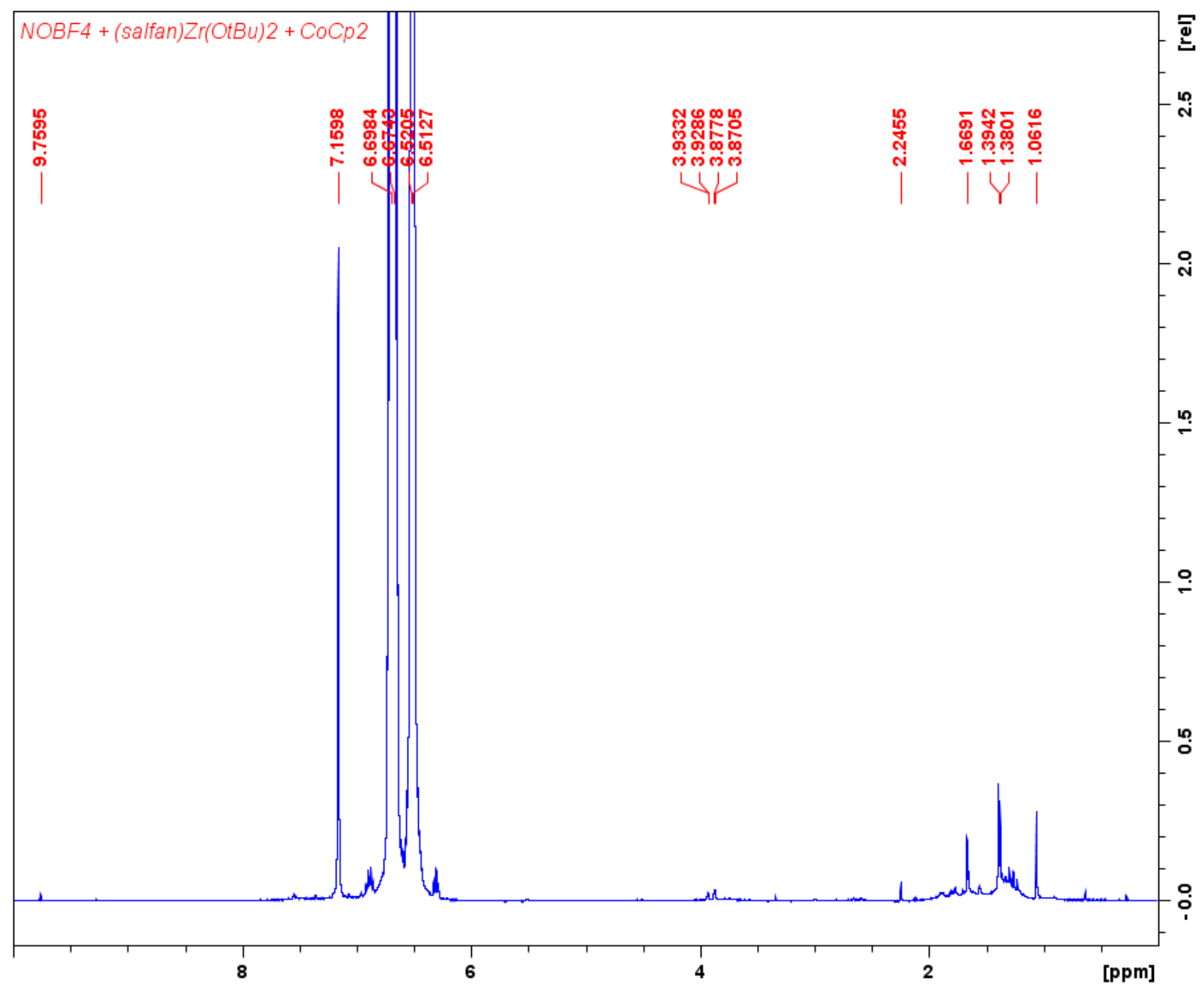

Figure S28. Table 3, entry 2. Oxidation of (salfan) $\mathrm{Zr}\left(\mathrm{O}^{t} \mathrm{Bu}\right)_{2}$ by $\mathrm{NOBF}_{4}$ and subsequent reduction by $\mathrm{CoCp}_{2} .{ }^{1} \mathrm{H}$ NMR $\left(300 \mathrm{MHz}, 25{ }^{\circ} \mathrm{C}, \mathrm{C}_{6} \mathrm{D}_{6}\right), \delta(\mathrm{ppm}): 9.76(\mathrm{~s}, 2 \mathrm{H}, \mathrm{OH}), 7.53(\mathrm{~m}, 2 \mathrm{H}$, $\mathrm{PhH}$ ), 6.68 (CFCH 1,2-difluorobenzene), 6.51 (CFCHCH 1,2-difluorobenzene), 3.93 (m, 2H, $\mathrm{CpH}), 3.88(\mathrm{~m}, 2 \mathrm{H}, \mathrm{CpH}), 2.25\left(\mathrm{~s}, 6 \mathrm{H}, \mathrm{NCH}_{3}\right), 1.66\left(\mathrm{~s}, 18 \mathrm{H}, \mathrm{C}\left(\mathrm{CH}_{3}\right)_{3}\right), 1.39(\mathrm{~s}, 18 \mathrm{H}$, $\left.\mathrm{C}\left(\mathrm{CH}_{3}\right)_{3}\right), 1.38\left(\mathrm{~s}, 18 \mathrm{H}, \mathrm{C}\left(\mathrm{CH}_{3}\right)_{3}\right), 1.06\left(\mathrm{~s}, 18 \mathrm{H}, \mathrm{C}\left(\mathrm{CH}_{3}\right)_{3}\right)$. 


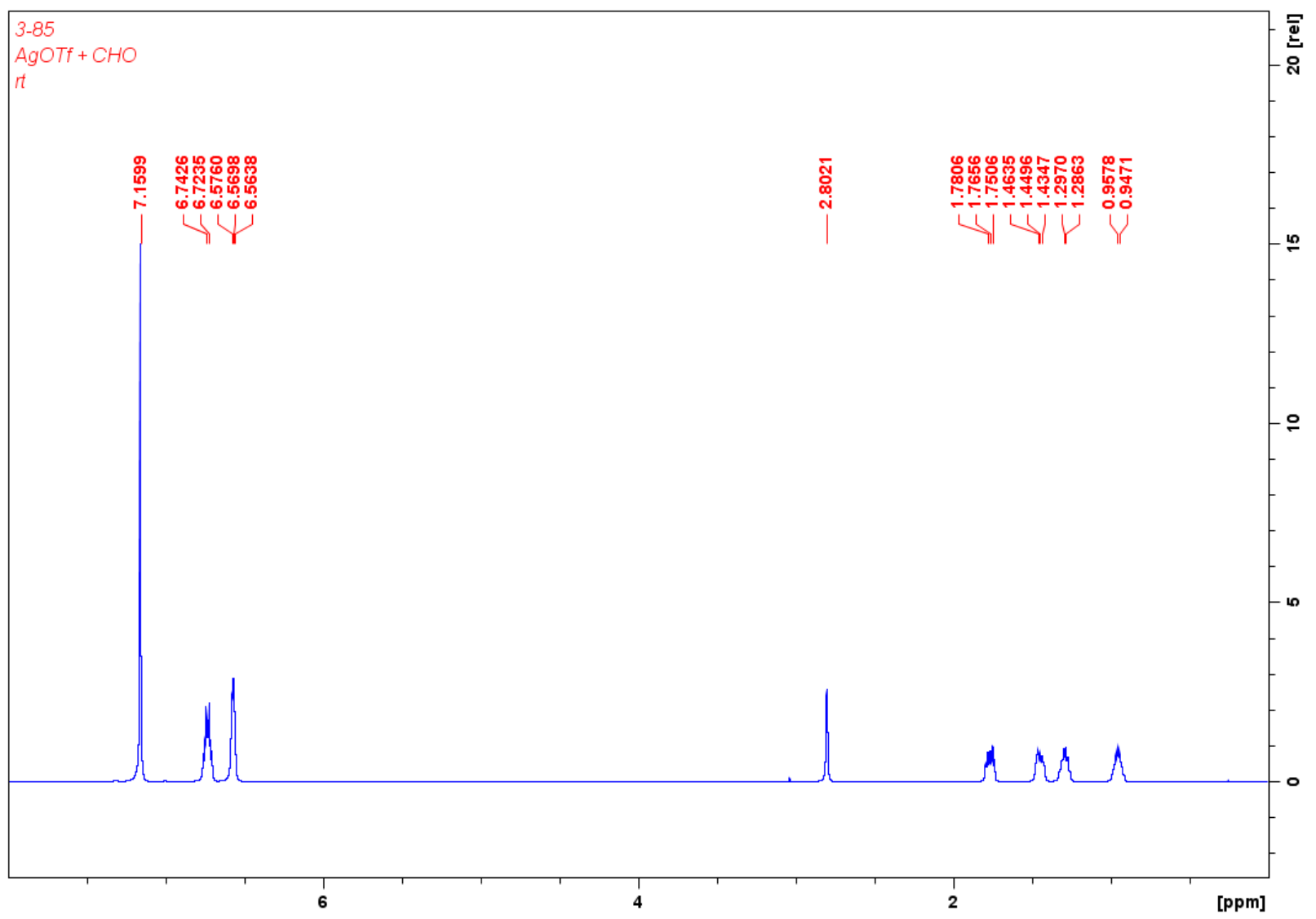

Figure S29. Table 3, entry 3. Polymerization of 100 equivalents of cyclohexene oxide by AgOTf at room temperature. ${ }^{1} \mathrm{H}$ NMR $\left(300 \mathrm{MHz}, 25{ }^{\circ} \mathrm{C}, \mathrm{C}_{6} \mathrm{D}_{6}\right), \delta(\mathrm{ppm}): 6.73$ (CFCH 1,2difluorobenzene), 6.55 ( $\mathrm{CFCHCH}$ 1,2-difluorobenzene), 2.80 (s, 2H, $\left.\mathrm{COCH}_{2} \mathrm{CHO}\right), 1.77$ (m, $2 \mathrm{H}, \mathrm{COCHCH}_{2} \mathrm{CHO}$ ), 1.48 (m, 2H, $\left.\mathrm{COCHCH}_{2} \mathrm{CHO}\right), 1.29$ (m, 2H, $\left.\mathrm{COCHCH}_{2} \mathrm{CH}_{2} \mathrm{CHO}\right), 0.93$ (m, $\left.2 \mathrm{H}, \mathrm{COCHCH}_{2} \mathrm{CH}_{2} \mathrm{CHO}\right)$. 


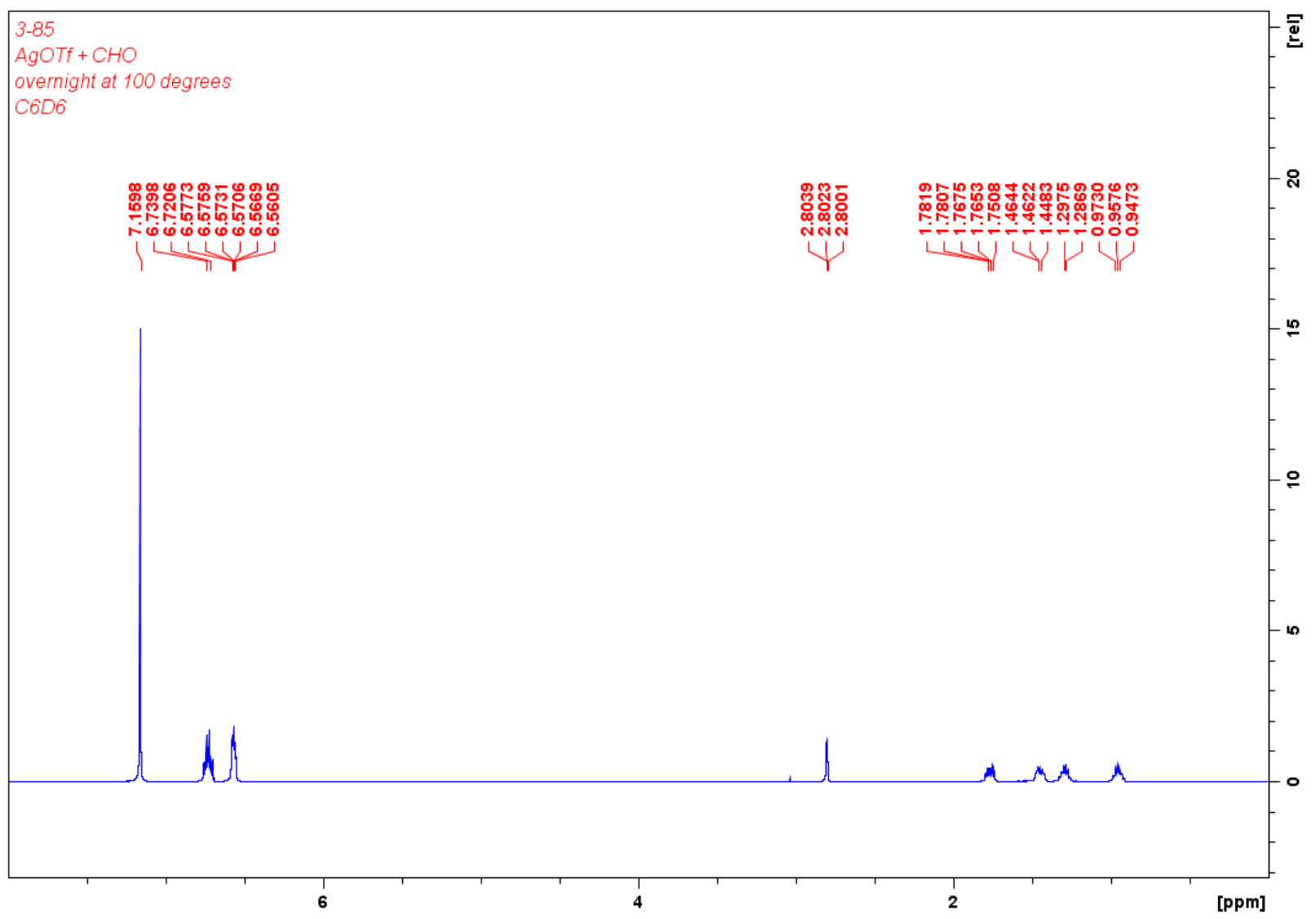

Figure S30. Table 3, entry 3. Polymerization of 100 equivalents of cyclohexene oxide by AgOTf at $100{ }^{\circ} \mathrm{C}$ overnight. ${ }^{1} \mathrm{H}$ NMR $\left(300 \mathrm{MHz}, 25{ }^{\circ} \mathrm{C}, \mathrm{C}_{6} \mathrm{D}_{6}\right), \delta(\mathrm{ppm}): 6.72$ (CFCH 1,2difluorobenzene), 6.57 ( $\mathrm{CFCHCH}$ 1,2-difluorobenzene), 2.80 (s, 2H, $\mathrm{COCH}_{2} \mathrm{CHO}$ ), 1.77 (m, $2 \mathrm{H}, \mathrm{COCHCH}_{2} \mathrm{CHO}$ ), 1.48 (m, 2H, $\left.\mathrm{COCHCH}_{2} \mathrm{CHO}\right), 1.29$ (m, 2H, $\left.\mathrm{COCHCH}_{2} \mathrm{CH}_{2} \mathrm{CHO}\right), 0.94$ (m, $\left.2 \mathrm{H}, \mathrm{COCHCH}_{2} \mathrm{CH}_{2} \mathrm{CHO}\right)$. 


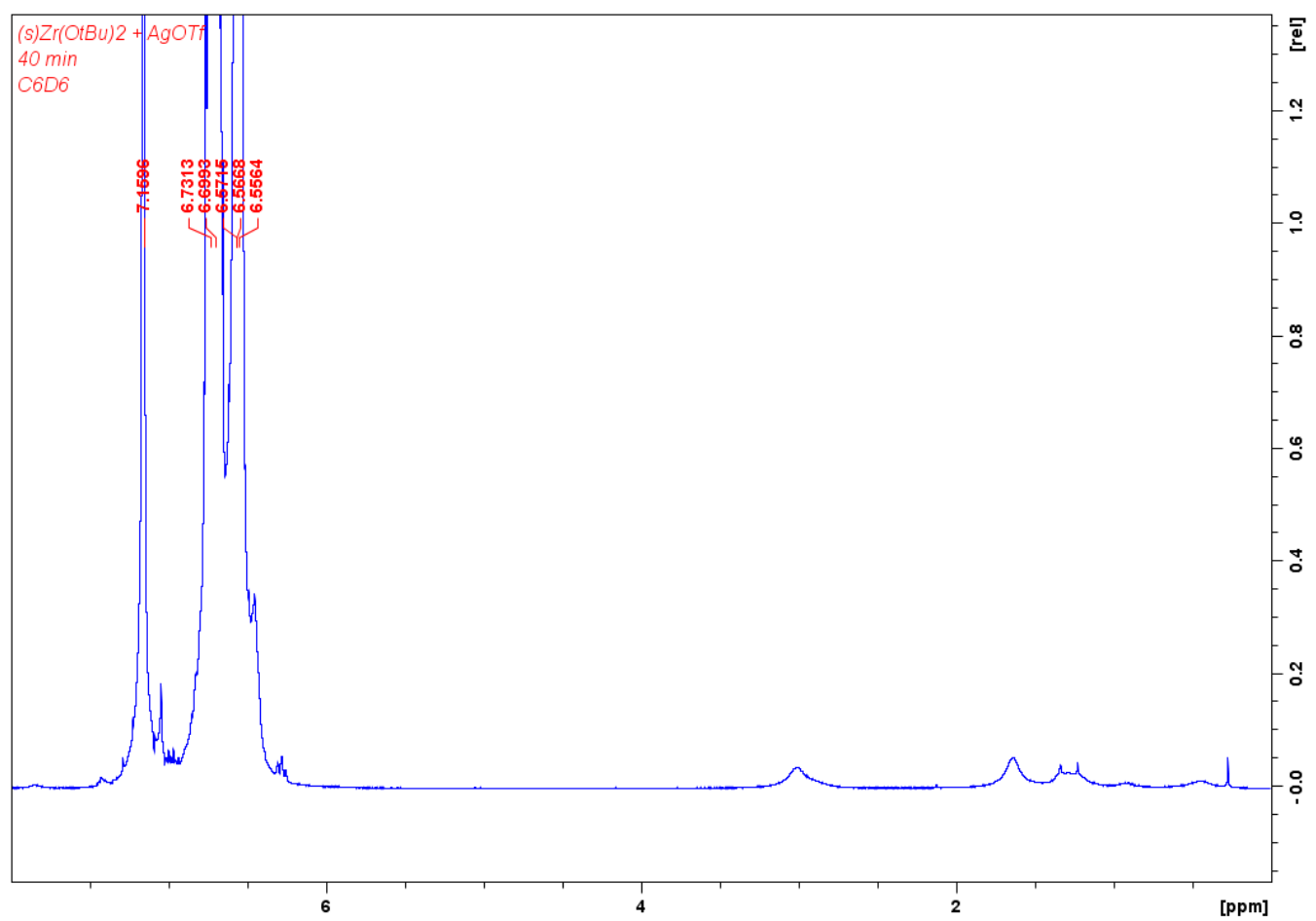

Figure S31. Table 3, entry 3. ${ }^{1} \mathrm{H}$ NMR $\left(300 \mathrm{MHz}, 25{ }^{\circ} \mathrm{C}, \mathrm{C}_{6} \mathrm{D}_{6}\right)$ spectrum of the oxidation of (salfan) $\mathrm{Zr}\left(\mathrm{O}^{t} \mathrm{Bu}\right)_{2}$ by AgOTf.

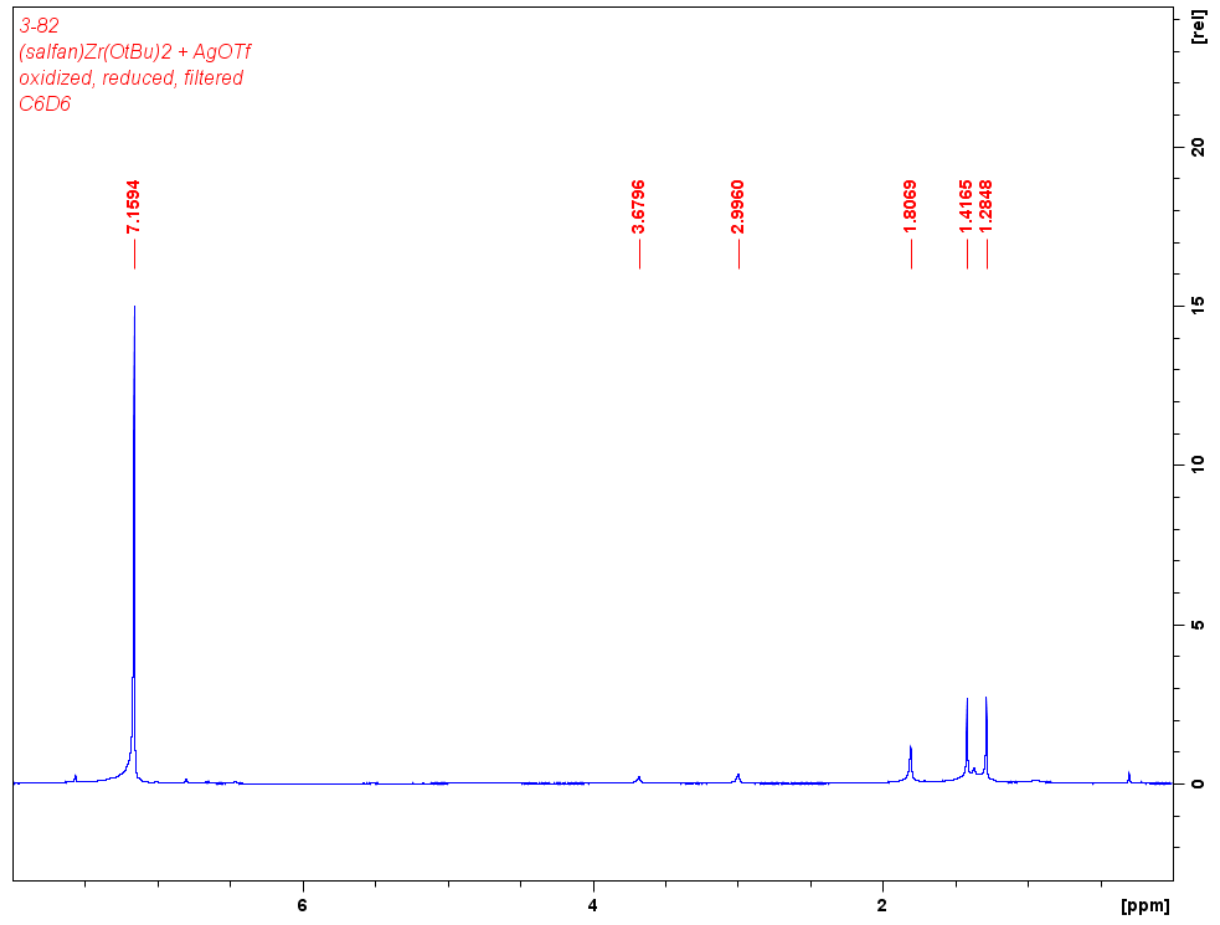

Figure S32. Table 3, entry 3. Oxidation of (salfan) $\mathrm{Zr}\left(\mathrm{O}^{t} \mathrm{Bu}\right)_{2}$ by $\mathrm{AgOTf}$ and subsequent reduction by $\mathrm{CoCp} 2 .{ }^{1} \mathrm{H}$ NMR $\left(300 \mathrm{MHz}, 25{ }^{\circ} \mathrm{C}, \mathrm{C}_{6} \mathrm{D}_{6}\right), \delta(\mathrm{ppm}): 3.67\left(\mathrm{~s}, 4 \mathrm{H}, \mathrm{NCH}_{2}\right), 2.99$ (s, $\left.6 \mathrm{H}, \mathrm{NCH}_{3}\right), 1.80\left(\mathrm{~s}, 18 \mathrm{H}, \mathrm{C}\left(\mathrm{CH}_{3}\right)_{3}\right), 1.41\left(\mathrm{~s}, 18 \mathrm{H}, \mathrm{C}\left(\mathrm{CH}_{3}\right)_{3}\right), 1.28\left(\mathrm{~s}, 18 \mathrm{H}, \mathrm{C}\left(\mathrm{CH}_{3}\right)_{3}\right)$. 


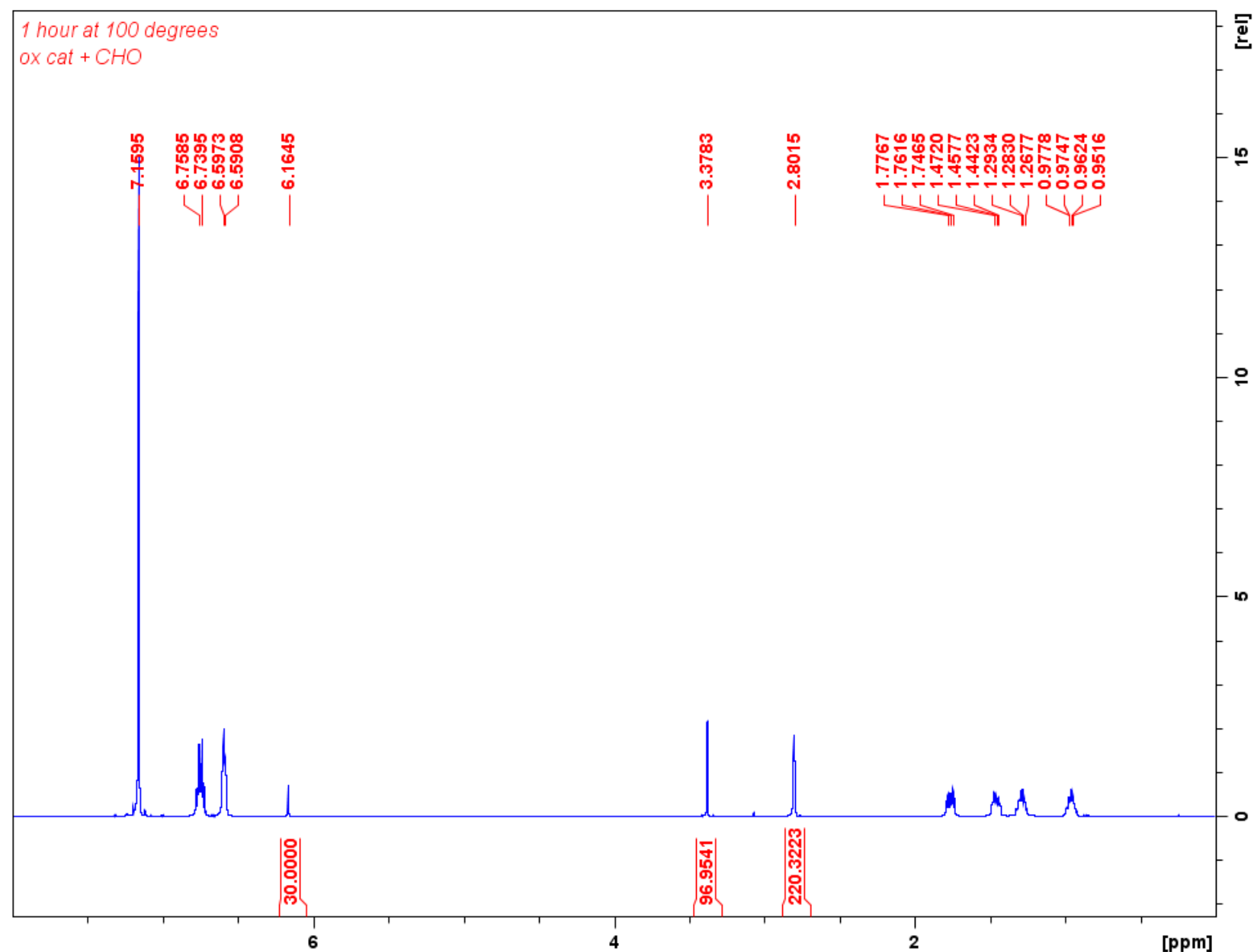

Figure S33. Table 3, entry 3. Polymerization of 100 equivalents of cyclohexene oxide by in situ generated [(salfan) $\left.\mathrm{Zr}\left(\mathrm{O}^{t} \mathrm{Bu}\right)_{2}\right][\mathrm{OTf}]$ using AgOTf. ${ }^{1} \mathrm{H}$ NMR $\left(300 \mathrm{MHz}, 25{ }^{\circ} \mathrm{C}, \mathrm{C}_{6} \mathrm{D}_{6}\right), \delta(\mathrm{ppm})$ : 6.74 (CFCH 1,2-difluorobenzene), 6.59 (CFCHCH 1,2-difluorobenzene), 6.16 (s, 3H, $\mathrm{PhH}$ TMB), 3.38 (s, 9H, CH3 TMB), 2.80 (s, 2H, $\mathrm{COCH}_{2} \mathrm{CHO}$ ), 1.77 (m, 2H, $\mathrm{COCHCH}_{2} \mathrm{CHO}$ ), 1.48 $\left(\mathrm{m}, 2 \mathrm{H}, \mathrm{COCHCH}_{2} \mathrm{CHO}\right), 1.28\left(\mathrm{~m}, 2 \mathrm{H}, \mathrm{COCHCH}_{2} \mathrm{CH}_{2} \mathrm{CHO}\right), 0.93\left(\mathrm{~m}, 2 \mathrm{H}, \mathrm{COCHCH}_{2} \mathrm{CH}_{2}\right.$ $\mathrm{CHO})$. 


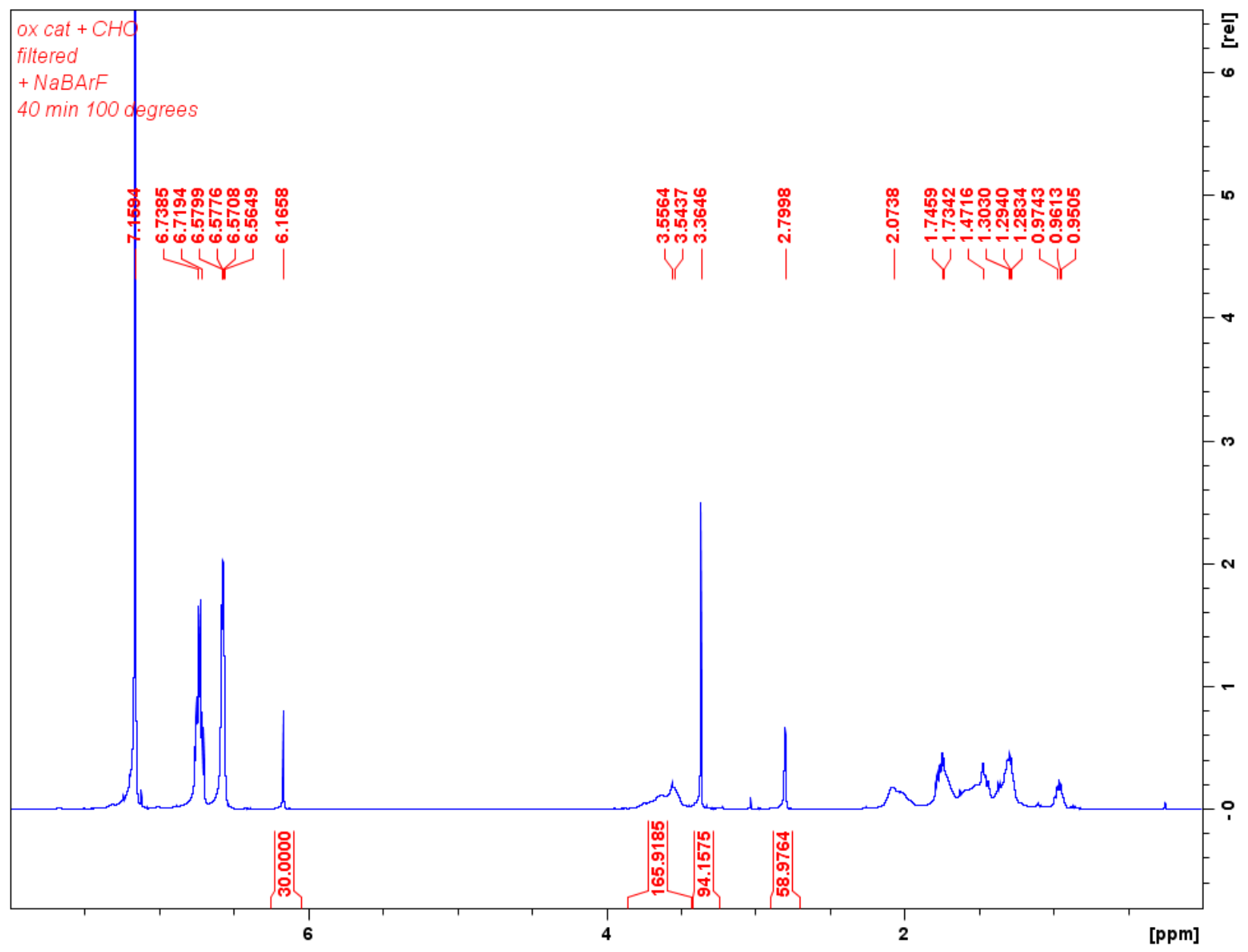

Figure S34. Table 3, entry 3. Polymerization of 100 equivalents of cyclohexene oxide by in situ generated [(salfan) $\left.\mathrm{Zr}\left(\mathrm{O}^{t} \mathrm{Bu}\right)_{2}\right][\mathrm{OTf}]$ using $\mathrm{AgOTf}$ in the presence of $\mathrm{Na}\left[\mathrm{BAr}{ }^{\mathrm{F}} 4\right] .{ }^{1} \mathrm{H} \mathrm{NMR}(300$ $\left.\mathrm{MHz}, 25{ }^{\circ} \mathrm{C}, \mathrm{C}_{6} \mathrm{D}_{6}\right), \delta(\mathrm{ppm}): 6.71$ (CFCH 1,2-difluorobenzene), 6.58 (CFCHCH 1,2difluorobenzene), 6.17 (s, 3H, $\mathrm{PhH} \mathrm{TMB}$ ), 3.55 (m, 2H, COCH PCHO), 3.36 (s, 9H, $\mathrm{CH}_{3} \mathrm{TMB}$ ), 2.80 (s, 2H, $\left.\mathrm{COCH}_{2} \mathrm{CHO}\right), 2.07$ (m, 2H, $\left.\mathrm{COCHCH}_{2} \mathrm{PCHO}\right), 1.74$ (m, 2H, COCHCH $2 \mathrm{CHO}$ ), $1.47\left(\mathrm{~m}, 2 \mathrm{H}, \mathrm{COCHCH}_{2} \mathrm{CH}_{2} \mathrm{CHO}\right), 1.30\left(\mathrm{~m}, 2 \mathrm{H}, \mathrm{COCHCH}_{2} \mathrm{CH}_{2} \mathrm{CHO}\right), 0.93(\mathrm{~m}, 2 \mathrm{H}$, $\mathrm{COCHCH}_{2} \mathrm{CH}_{2} \mathrm{CHO}$ ). 


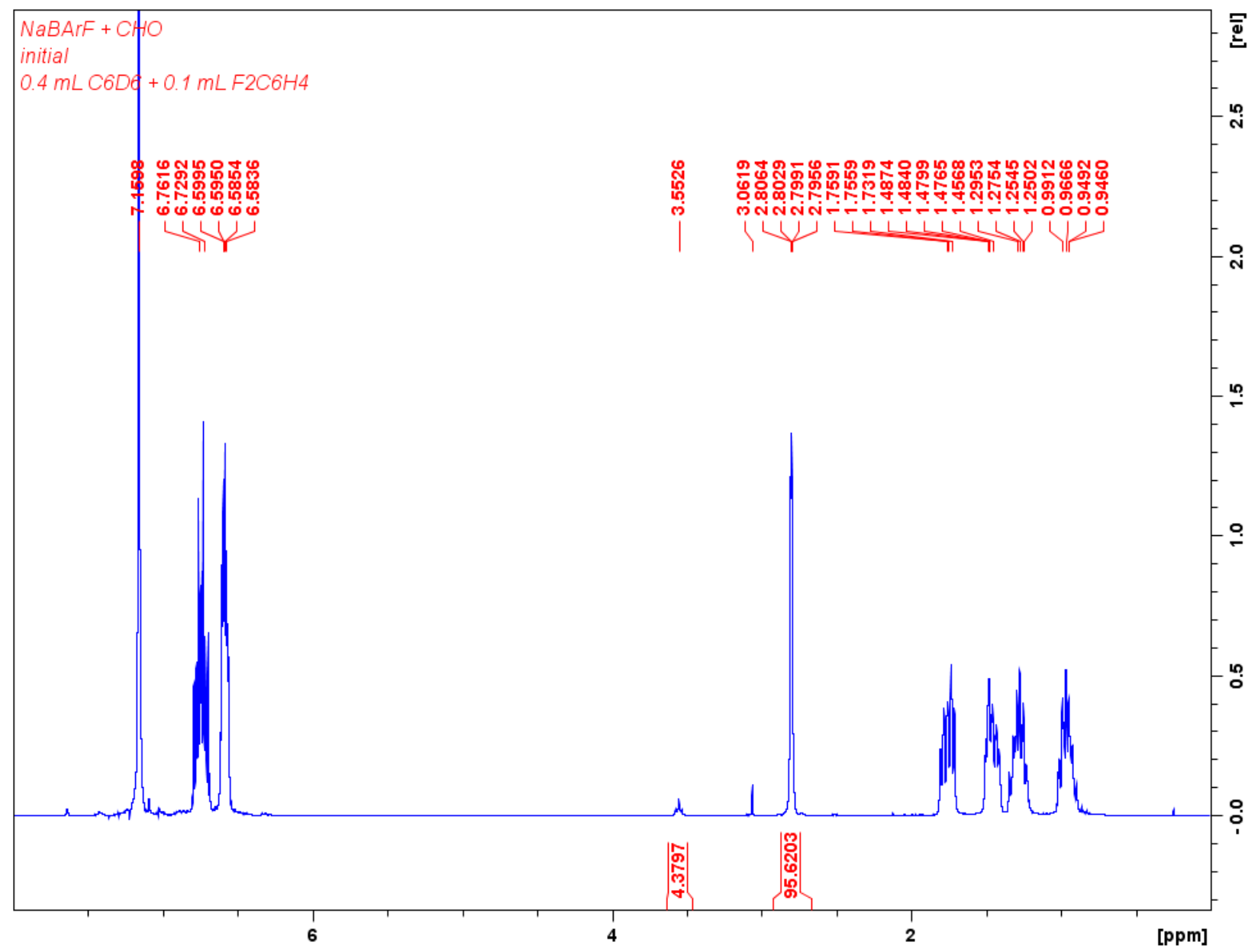

Figure S35. Table 3, entry 4. Polymerization of 100 equivalents of cyclohexene oxide by $\mathrm{Na}\left[\mathrm{BAr}_{4}{ }_{4}\right]$ at ambient temperature. ${ }^{1} \mathrm{H} \mathrm{NMR}\left(300 \mathrm{MHz}, 25{ }^{\circ} \mathrm{C}, \mathrm{C}_{6} \mathrm{D}_{6}\right), \delta(\mathrm{ppm}): 6.73$ (CFCH 1,2difluorobenzene), 6.59 (CFCHCH 1,2-difluorobenzene), 3.55 (m, 2H, COCH PCHO), 2.80 (s, $\left.2 \mathrm{H}, \mathrm{COCH}_{2} \mathrm{CHO}\right), 1.75\left(\mathrm{~m}, 2 \mathrm{H}, \mathrm{COCHCH}_{2} \mathrm{CHO}\right), 1.48\left(\mathrm{~m}, 2 \mathrm{H}, \mathrm{COCHCH}_{2} \mathrm{CHO}\right), 1.28(\mathrm{~m}$, $\left.2 \mathrm{H}, \mathrm{COCHCH}_{2} \mathrm{CH}_{2} \mathrm{CHO}\right), 0.95$ (m, $\left.2 \mathrm{H}, \mathrm{COCHCH}_{2} \mathrm{CH}_{2} \mathrm{CHO}\right)$. 


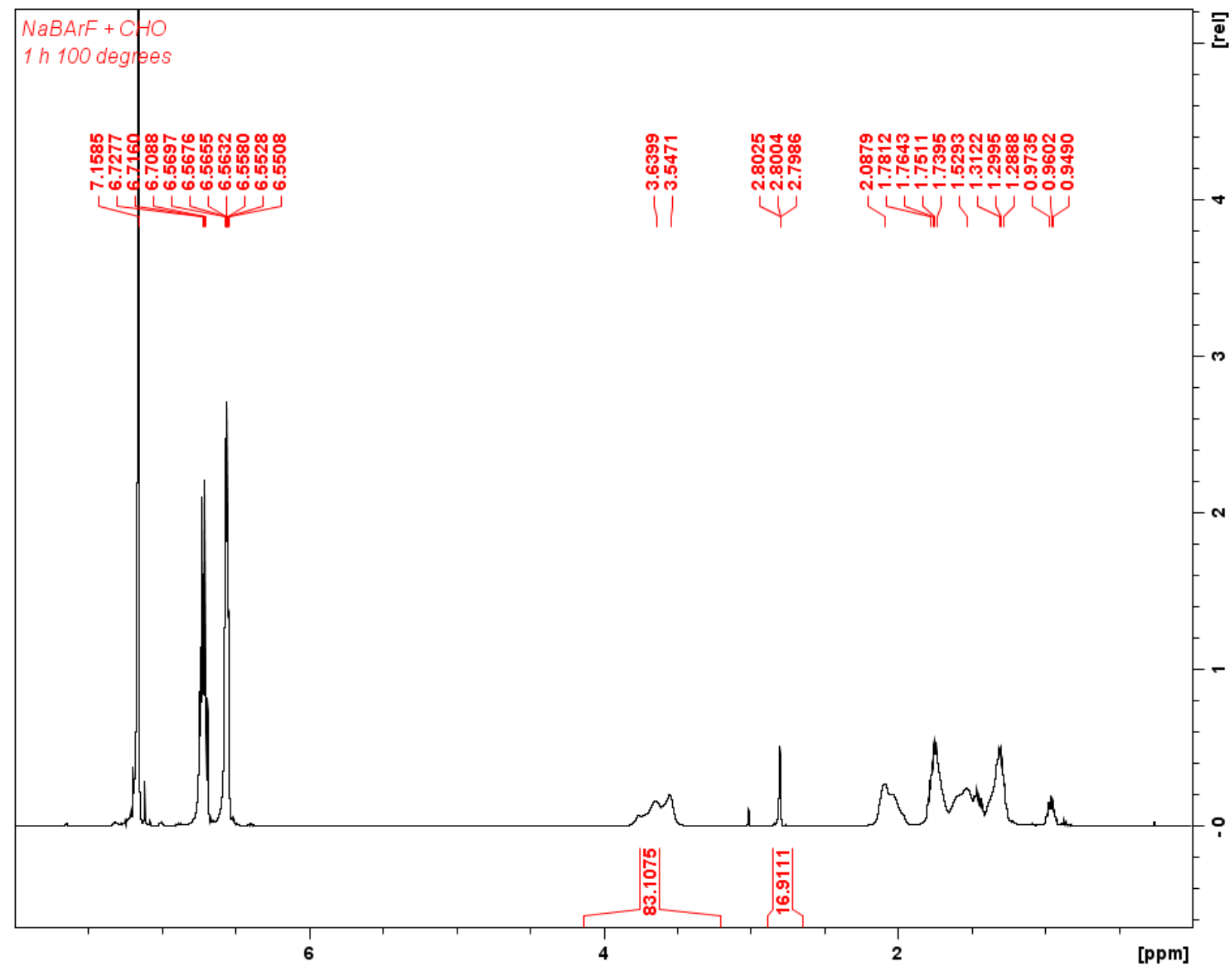

Figure S36. Table 3, entry 4. Polymerization of 100 equivalents of cyclohexene oxide by $\mathrm{Na}\left[\mathrm{BAr}^{\mathrm{F}} 4\right.$ ] after $1 \mathrm{~h}$ at $100{ }^{\circ} \mathrm{C} .{ }^{1} \mathrm{H}$ NMR $\left(300 \mathrm{MHz}, 25{ }^{\circ} \mathrm{C}, \mathrm{C}_{6} \mathrm{D} 6\right), \delta(\mathrm{ppm}): 6.71(\mathrm{CFCH}$ 1,2difluorobenzene), 6.55 (CFCHCH 1,2-difluorobenzene), 3.56 (m, 2H, COCH PCHO), 2.80 (s, $2 \mathrm{H}, \mathrm{COCH}_{2} \mathrm{CHO}$ ), 2.08 (m, 2H, $\left.\mathrm{COCHCH}_{2} \mathrm{PCHO}\right), 1.74$ (m, 2H, $\left.\mathrm{COCHCH}_{2} \mathrm{PCHO}\right), 1.53$ (m, $\left.2 \mathrm{H}, \mathrm{COCHCH}_{2} \mathrm{CHO}\right), 1.47$ (m, 2H, $\left.\mathrm{COCHCH}_{2} \mathrm{CHO}\right), 1.30$ (m, 2H, $\mathrm{COCHCH}_{2} \mathrm{CH}_{2} \mathrm{PCHO}$ ), $0.96\left(\mathrm{~m}, 2 \mathrm{H}, \mathrm{COCHCH}_{2} \mathrm{CH}_{2} \mathrm{PCHO}\right)$. 


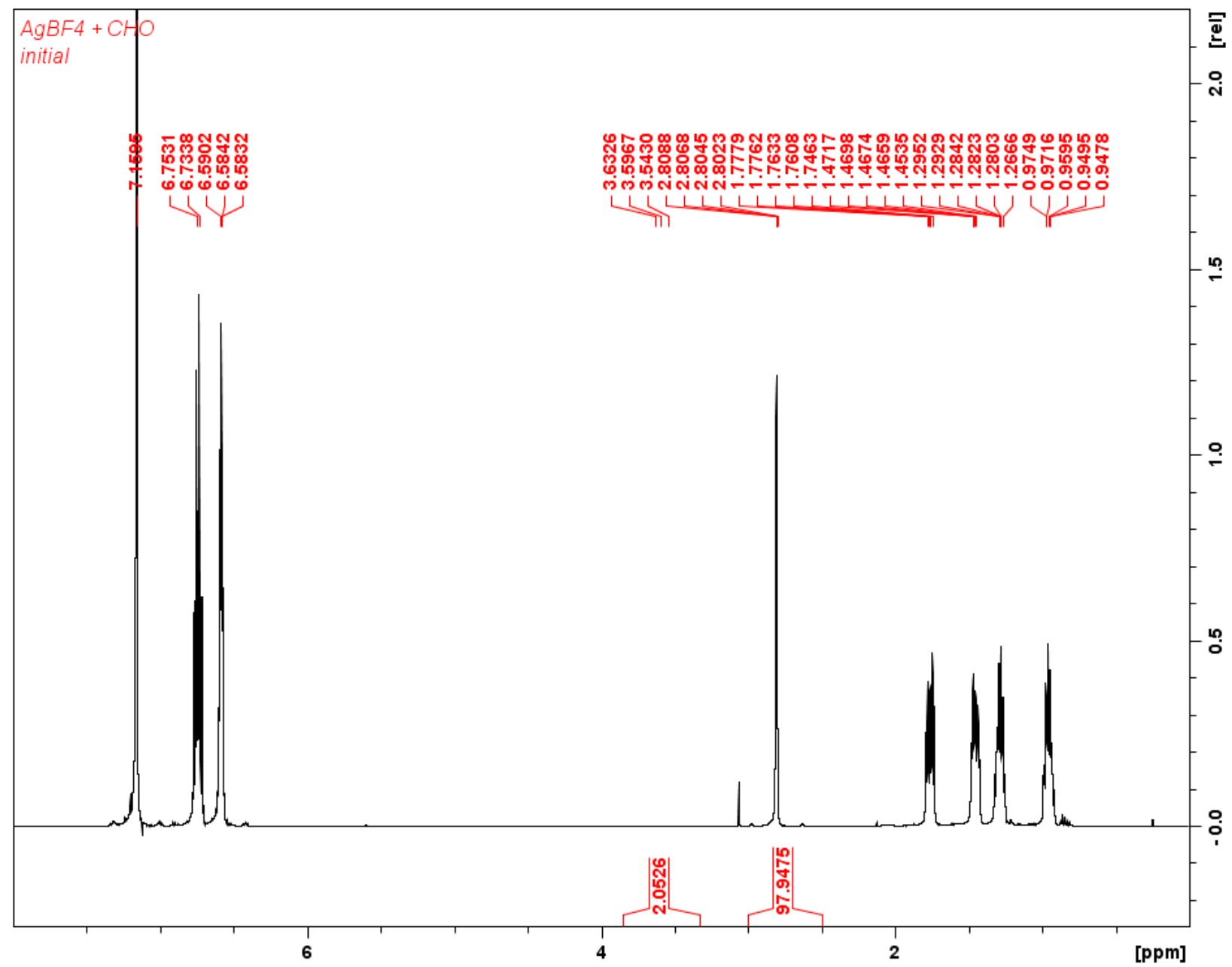

Figure S37. Table 3, entry 5. Polymerization of 100 equivalents of cyclohexene oxide by $\mathrm{AgBF}_{4}$ at ambient temperature. ${ }^{1} \mathrm{H}$ NMR $\left(300 \mathrm{MHz}, 25{ }^{\circ} \mathrm{C}, \mathrm{C}_{6} \mathrm{D}_{6}\right), \delta(\mathrm{ppm}): 6.74(\mathrm{CFCH} 1,2-$ difluorobenzene), 6.58 (CFCHCH 1,2-difluorobenzene), 3.60 (m, 2H, COCH PCHO), 2.81 (s, $\left.2 \mathrm{H}, \mathrm{COCH}_{2} \mathrm{CHO}\right), 1.77\left(\mathrm{~m}, 2 \mathrm{H}, \mathrm{COCHCH}_{2} \mathrm{CHO}\right), 1.48\left(\mathrm{~m}, 2 \mathrm{H}, \mathrm{COCHCH}_{2} \mathrm{CHO}\right), 1.28(\mathrm{~m}$, $\left.2 \mathrm{H}, \mathrm{COCHCH}_{2} \mathrm{CH}_{2} \mathrm{CHO}\right), 0.95$ (m, $\left.2 \mathrm{H}, \mathrm{COCHCH}_{2} \mathrm{CH}_{2} \mathrm{CHO}\right)$. 


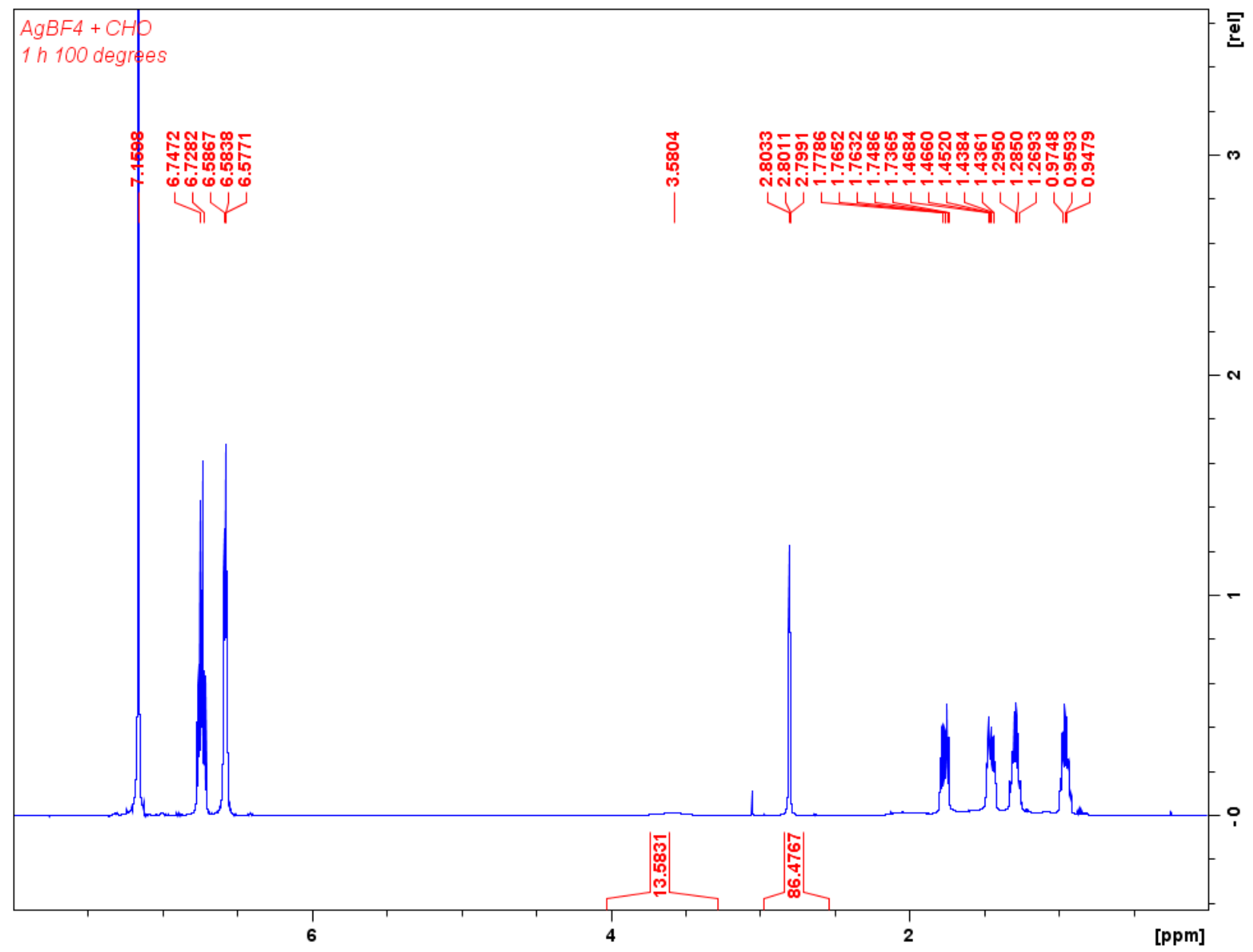

Figure S38. Table 3, entry 5. Polymerization of 100 equivalents of cyclohexene oxide by $\mathrm{AgBF}_{4}$ after $1 \mathrm{~h}$ at $100{ }^{\circ} \mathrm{C}$. ${ }^{1} \mathrm{H}$ NMR $\left(300 \mathrm{MHz}, 25{ }^{\circ} \mathrm{C}, \mathrm{C}_{6} \mathrm{D}_{6}\right), \delta(\mathrm{ppm}): 6.73$ (CFCH 1,2difluorobenzene), 6.58 (CFCHCH 1,2-difluorobenzene), 3.58 (m, 2H, COCH PCHO), 2.80 (s, $\left.2 \mathrm{H}, \mathrm{COCH}_{2} \mathrm{CHO}\right), 1.77\left(\mathrm{~m}, 2 \mathrm{H}, \mathrm{COCHCH}_{2} \mathrm{PCHO}\right), 1.47\left(\mathrm{~m}, 2 \mathrm{H}, \mathrm{COCHCH}_{2} \mathrm{CHO}\right), 1.29$ (m, $\left.2 \mathrm{H}, \mathrm{COCHCH}_{2} \mathrm{CH}_{2} \mathrm{CHO}\right), 0.95$ (m, 2H, $\left.\mathrm{COCHCH}_{2} \mathrm{CH}_{2} \mathrm{CHO}\right)$. 


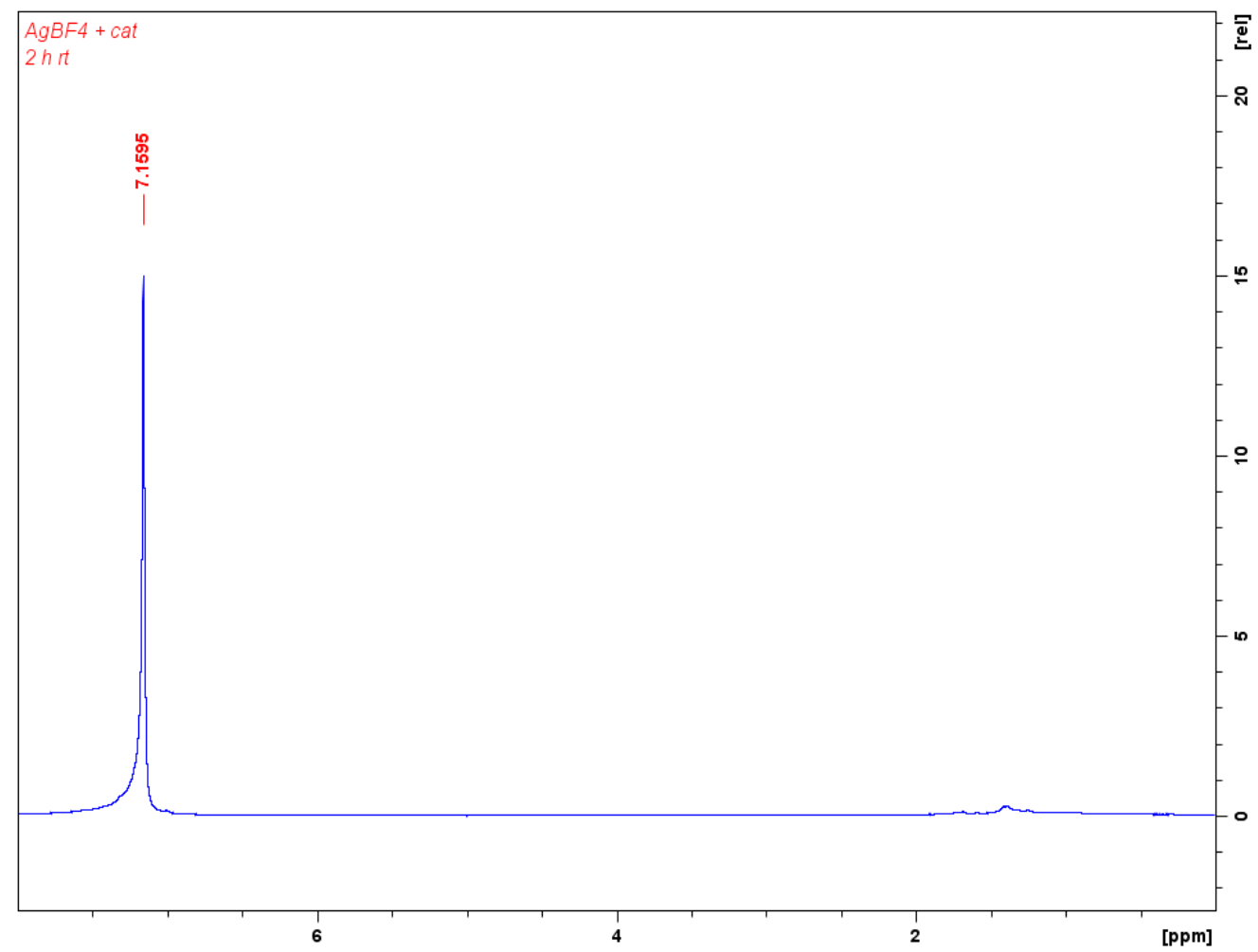

Figure S39. Table 3, entry 5. ${ }^{1} \mathrm{H}$ NMR $\left(300 \mathrm{MHz}, 25{ }^{\circ} \mathrm{C}, \mathrm{C}_{6} \mathrm{D}_{6}\right)$ spectrum of the oxidation of (salfan) $\mathrm{Zr}\left(\mathrm{O}^{t} \mathrm{Bu}\right)_{2}$ by $\mathrm{AgBF}_{4}$.

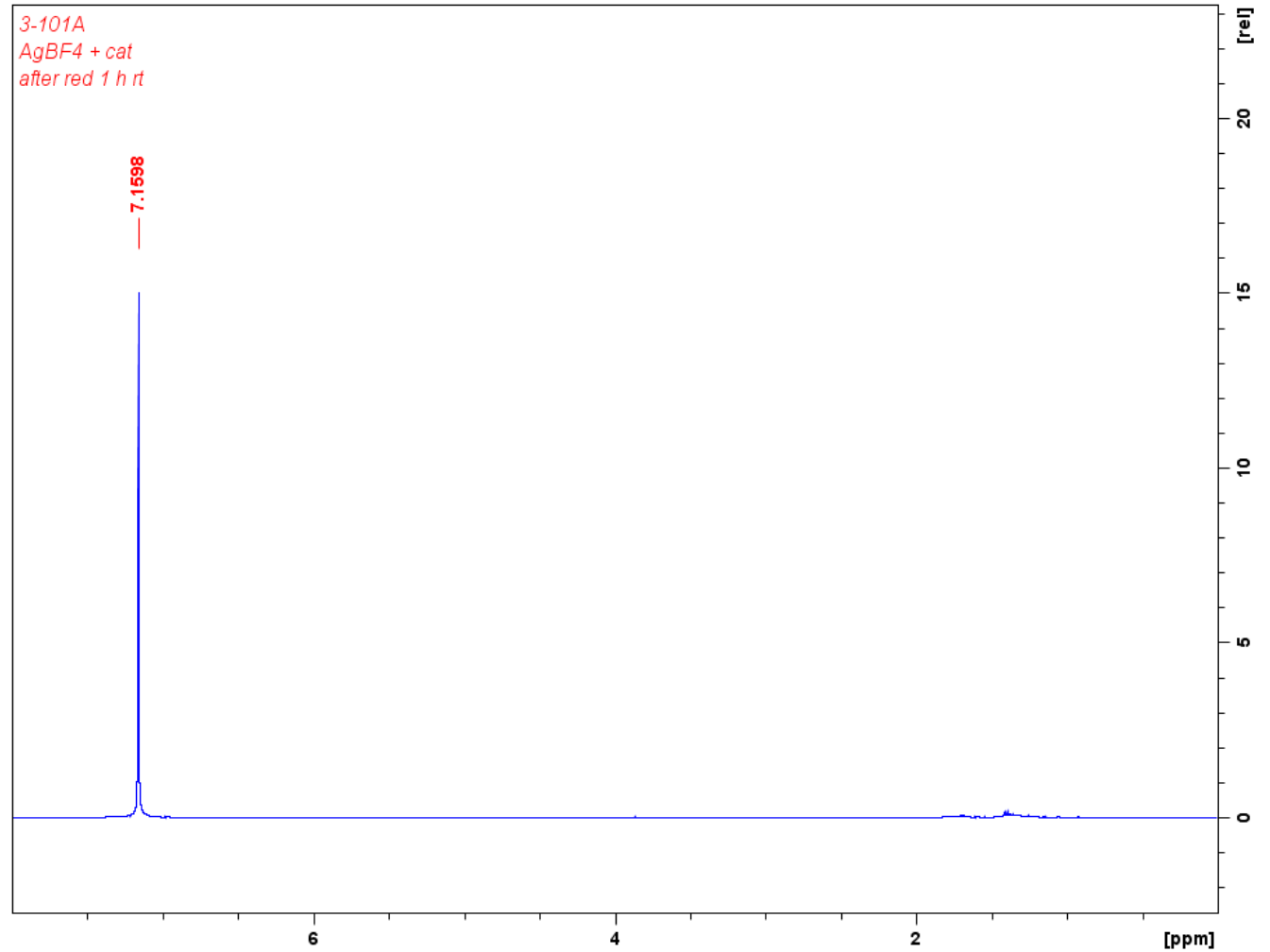

Figure S40. Table 3, entry 5. ${ }^{1} \mathrm{H}$ NMR $\left(300 \mathrm{MHz}, 25{ }^{\circ} \mathrm{C}, \mathrm{C}_{6} \mathrm{D}_{6}\right)$ spectrum of the oxidation of (salfan) $\mathrm{Zr}\left(\mathrm{O}^{t} \mathrm{Bu}\right)_{2}$ by $\mathrm{AgBF}_{4}$ and subsequent reduction by $\mathrm{CoCp}_{2}$. 


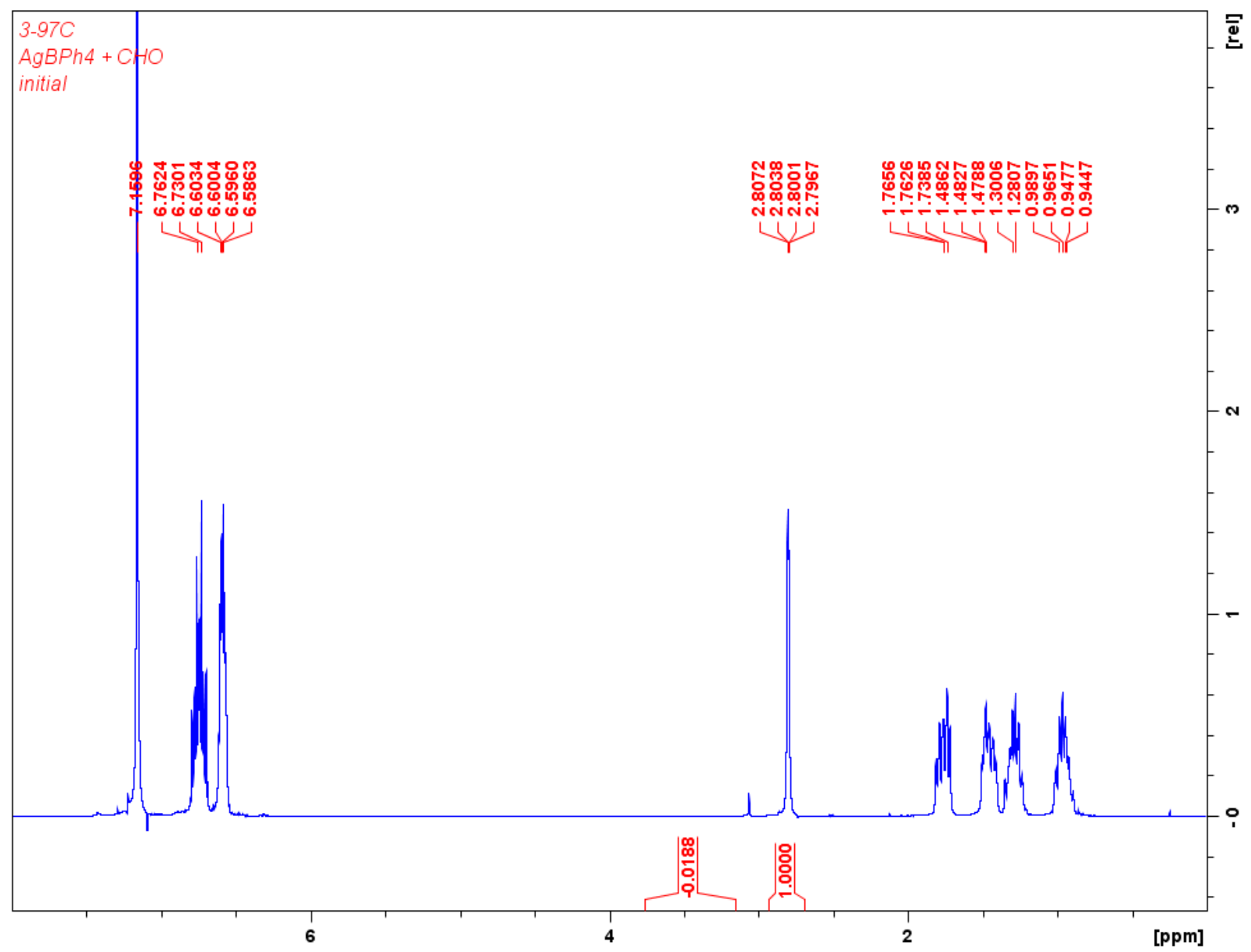

Figure S41. Table 3, entry 6. Polymerization of 100 equivalents of cyclohexene oxide by $\mathrm{AgBPh}_{4}$ at ambient temperature. ${ }^{1} \mathrm{H}$ NMR $\left(300 \mathrm{MHz}, 25{ }^{\circ} \mathrm{C}, \mathrm{C}_{6} \mathrm{D}_{6}\right), \delta(\mathrm{ppm}): 6.71(\mathrm{CFCH} 1,2-$ difluorobenzene), 6.55 (CFCHCH 1,2-difluorobenzene), 3.60 area (m, 2H, COCH PCHO), 2.80 (s, $\left.2 \mathrm{H}, \mathrm{COCH}_{2} \mathrm{CHO}\right), 1.76\left(\mathrm{~m}, 2 \mathrm{H}, \mathrm{COCHCH}_{2} \mathrm{CHO}\right), 1.48\left(\mathrm{~m}, 2 \mathrm{H}, \mathrm{COCHCH}_{2} \mathrm{CHO}\right), 1.29(\mathrm{~m}$, $\left.2 \mathrm{H}, \mathrm{COCHCH}_{2} \mathrm{CH}_{2} \mathrm{CHO}\right), 0.95$ (m, $\left.2 \mathrm{H}, \mathrm{COCHCH}_{2} \mathrm{CH}_{2} \mathrm{CHO}\right)$. 


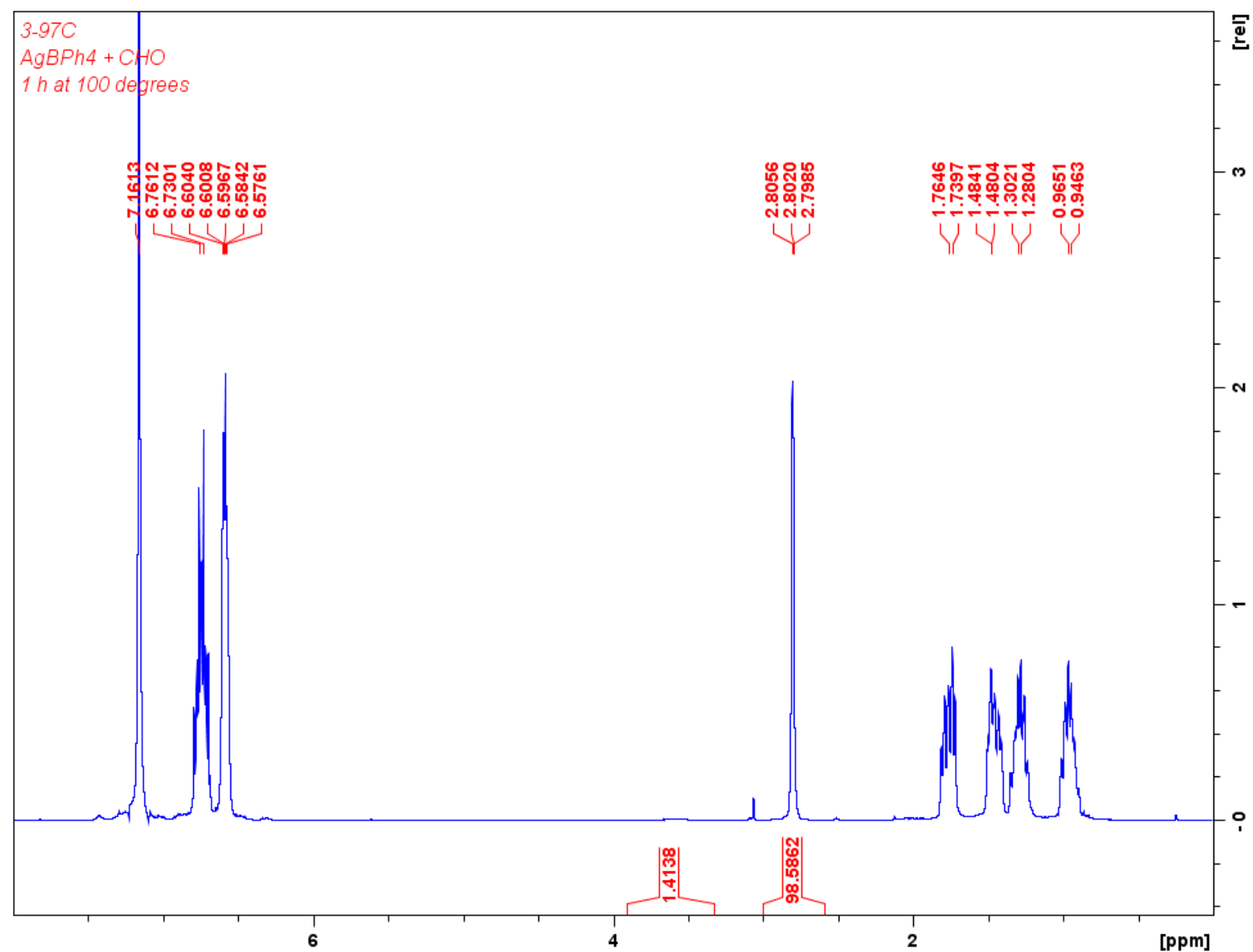

Figure S42. Table 3, entry 6. Polymerization of 100 equivalents of cyclohexene oxide by $\mathrm{AgBPh}_{4}$ after $1 \mathrm{~h}$ at $100{ }^{\circ} \mathrm{C}$. ${ }^{1} \mathrm{H}$ NMR $\left(300 \mathrm{MHz}, 25{ }^{\circ} \mathrm{C}, \mathrm{C}_{6} \mathrm{D}_{6}\right) \delta(\mathrm{ppm}): 6.73(\mathrm{CFCH} 1,2-$ difluorobenzene), 6.60 ( $\mathrm{CFCHCH}$ 1,2-difluorobenzene), $3.62(\mathrm{~m}, 2 \mathrm{H}, \mathrm{COCH} \mathrm{PCHO}), 2.80$ (s, $\left.2 \mathrm{H}, \mathrm{COCH}_{2} \mathrm{CHO}\right), 1.75\left(\mathrm{~m}, 2 \mathrm{H}, \mathrm{COCHCH}_{2} \mathrm{CHO}\right), 1.48\left(\mathrm{~m}, 2 \mathrm{H}, \mathrm{COCHCH}_{2} \mathrm{CHO}\right), 1.29$ (m, $\left.2 \mathrm{H}, \mathrm{COCHCH}_{2} \mathrm{CH}_{2} \mathrm{CHO}\right), 0.95$ (m, 2H, $\left.\mathrm{COCHCH}_{2} \mathrm{CH}_{2} \mathrm{CHO}\right)$. 


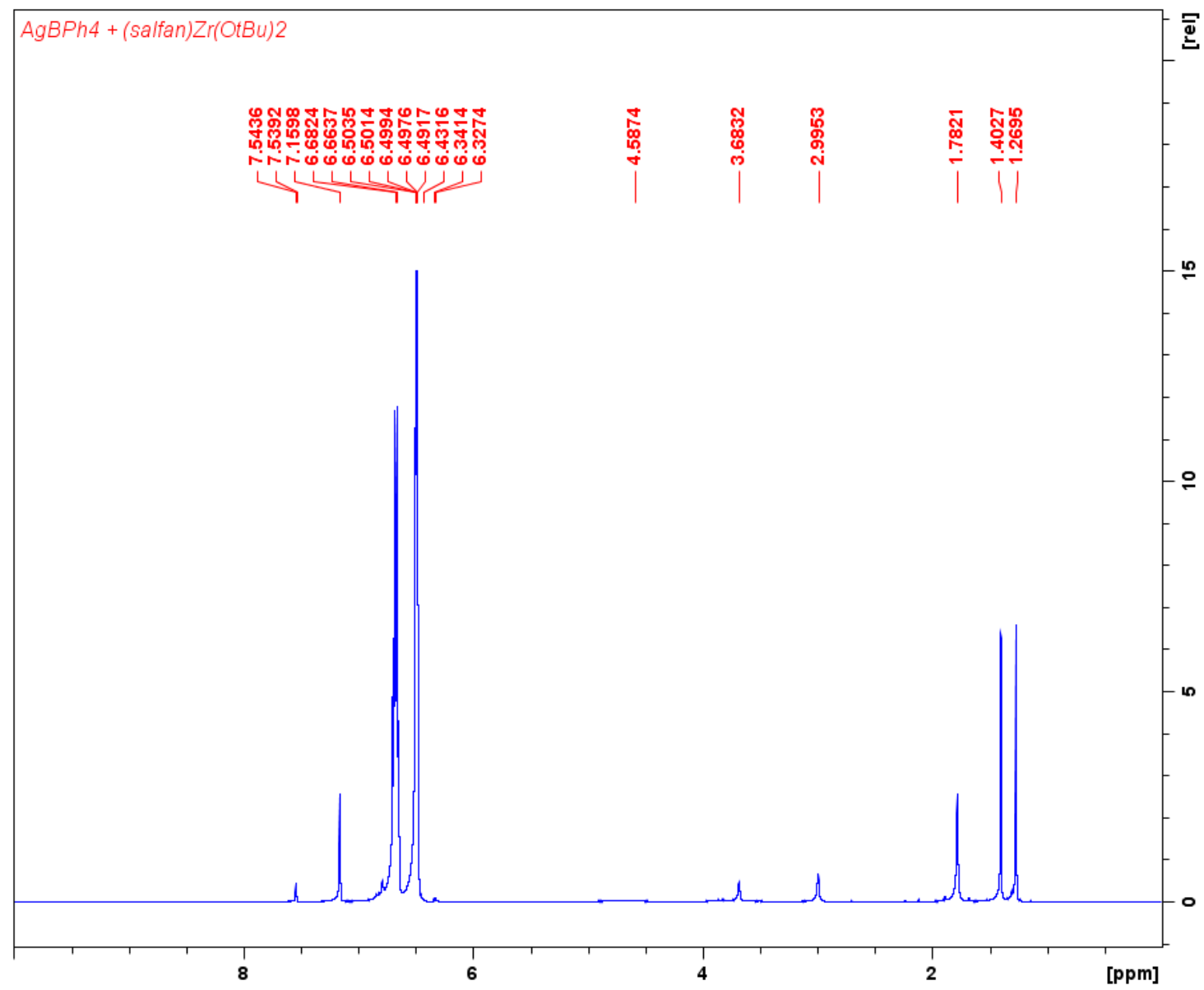

Figure S43. Table 3, entry 6. Oxidation of (salfan) $\mathrm{Zr}\left(\mathrm{O}^{t} \mathrm{Bu}\right)_{2}$ by $\mathrm{AgBPh} 4 .{ }^{1} \mathrm{H} \mathrm{NMR}(300 \mathrm{MHz}$, $\left.25{ }^{\circ} \mathrm{C}, \mathrm{C}_{6} \mathrm{D}_{6}\right), \delta(\mathrm{ppm}): 7.54(\mathrm{~d}, \mathrm{~J}=2.5 \mathrm{~Hz}, 2 \mathrm{H}, \mathrm{PhH}), 6.79$ (d, J = 2.5 Hz, 2H, PhH), $6.67(\mathrm{CFCH}$ 1,2-difluorobenzene), 6.50 (CFCHCH 1,2-difluorobenzene), 4.59 (br, 2H, CpH), 3.68 (s, 4H, $\left.\mathrm{NCH}_{2}\right), 2.99\left(\mathrm{~s}, 6 \mathrm{H}, \mathrm{NCH}_{3}\right), 1.78\left(\mathrm{~s}, 18 \mathrm{H}, \mathrm{C}\left(\mathrm{CH}_{3}\right)_{3}\right), 1.40\left(\mathrm{~s}, 18 \mathrm{H}, \mathrm{C}\left(\mathrm{CH}_{3}\right)_{3}\right), 1.27(\mathrm{~s}, 18 \mathrm{H}$, $\left.\mathrm{C}\left(\mathrm{CH}_{3}\right)_{3}\right)$. 


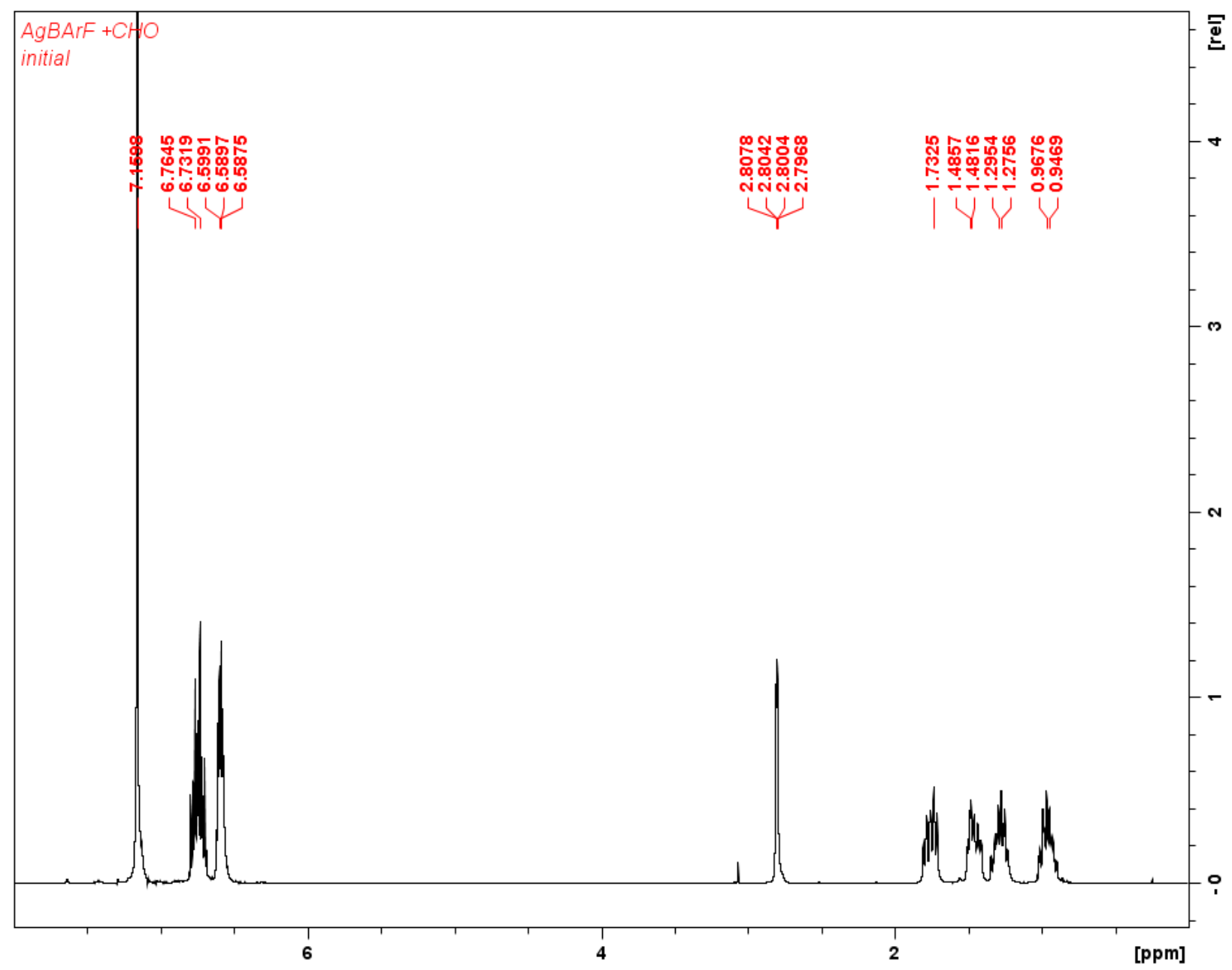

Figure S44. Table 3, entry 7. Polymerization of 100 equivalents of cyclohexene oxide by $\mathrm{Ag}\left[\mathrm{BAr}^{\mathrm{F}} 4\right.$ ] at ambient temperature. ${ }^{1} \mathrm{H} \mathrm{NMR}\left(300 \mathrm{MHz}, 25{ }^{\circ} \mathrm{C}, \mathrm{C}_{6} \mathrm{D}_{6}\right), \delta(\mathrm{ppm}): 6.76(\mathrm{CFCH} 1,2-$ difluorobenzene), 6.59 (CFCHCH 1,2-difluorobenzene), 2.80 (s, 2H, $\left.\mathrm{COCH}_{2} \mathrm{CHO}\right), 1.73(\mathrm{~m}$, $\left.2 \mathrm{H}, \mathrm{COCHCH}_{2} \mathrm{CHO}\right), 1.48\left(\mathrm{~m}, 2 \mathrm{H}, \mathrm{COCHCH}_{2} \mathrm{CHO}\right), 1.28\left(\mathrm{~m}, 2 \mathrm{H}, \mathrm{COCHCH}_{2} \mathrm{CH}_{2} \mathrm{CHO}\right), 0.95$ (m, $\left.2 \mathrm{H}, \mathrm{COCHCH}_{2} \mathrm{CH}_{2} \mathrm{CHO}\right)$. 


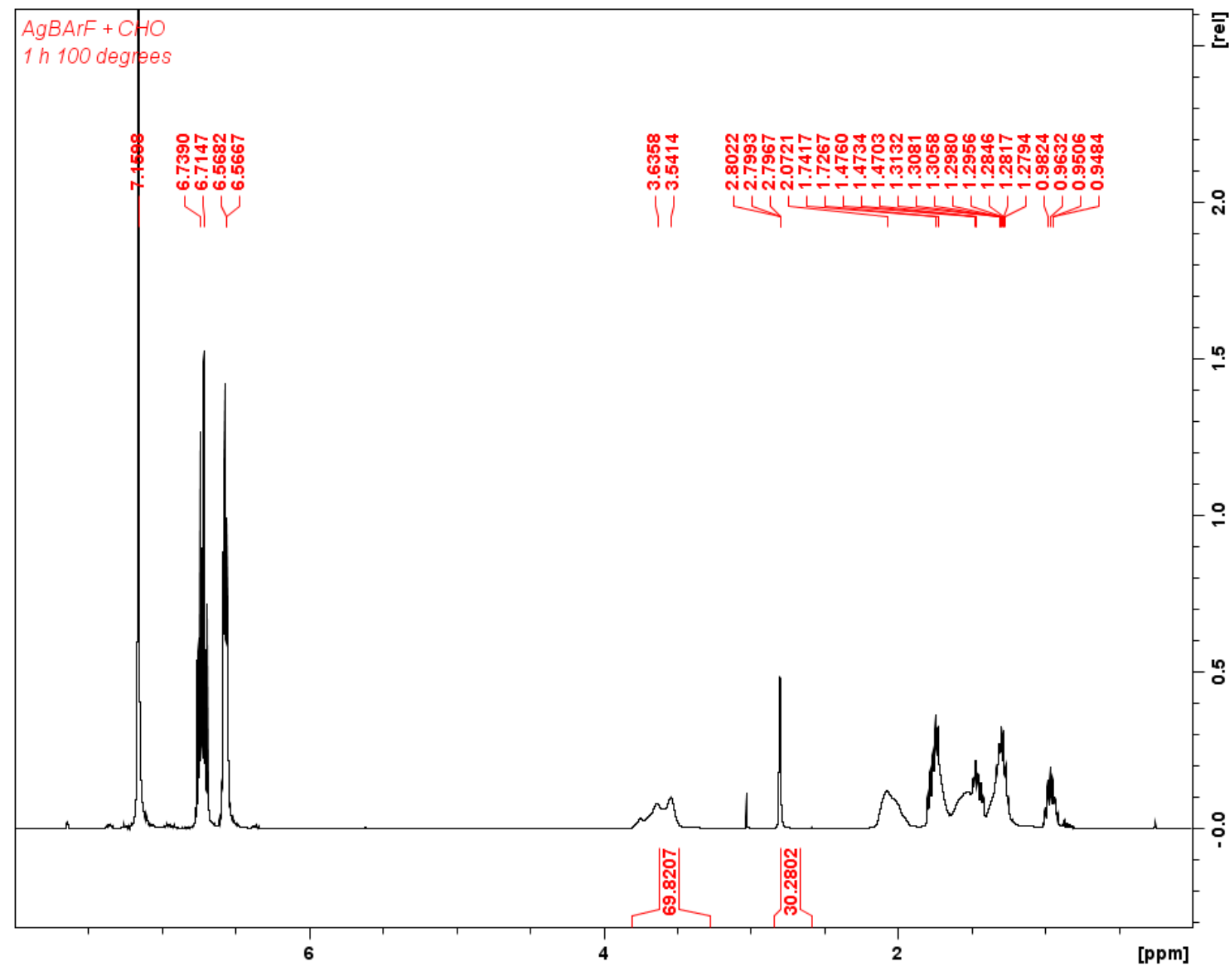

Figure S45. Table 3, entry 7. Polymerization of 100 equivalents of cyclohexene oxide by $\operatorname{Ag}\left[\mathrm{BAr}^{\mathrm{F}}{ }_{4}\right]$ after $1 \mathrm{~h}$ at $100{ }^{\circ} \mathrm{C} .{ }^{1} \mathrm{H}$ NMR $\left(300 \mathrm{MHz}, 25{ }^{\circ} \mathrm{C}, \mathrm{C}_{6} \mathrm{D} 6\right), \delta(\mathrm{ppm}): 6.72(\mathrm{CFCH}$ 1,2difluorobenzene), 6.57 (CFCHCH 1,2-difluorobenzene), $3.62(\mathrm{~m}, 2 \mathrm{H}, \mathrm{COCH} \mathrm{PCHO}), 2.80$ (s, $\left.2 \mathrm{H}, \mathrm{COCH}_{2} \mathrm{CHO}\right), 2.07\left(\mathrm{~m}, 2 \mathrm{H}, \mathrm{COCHCH}_{2} \mathrm{PCHO}\right), 1.74\left(\mathrm{~m}, 2 \mathrm{H}, \mathrm{COCHCH}_{2} \mathrm{CHO}\right), 1.47$ (m, $\left.2 \mathrm{H}, \mathrm{COCHCH}_{2} \mathrm{CHO}\right), 1.28\left(\mathrm{~m}, 2 \mathrm{H}, \mathrm{COCHCH}_{2} \mathrm{CH}_{2} \mathrm{CHO}\right), 0.95\left(\mathrm{~m}, 2 \mathrm{H}, \mathrm{COCHCH}_{2} \mathrm{CH}_{2} \mathrm{CHO}\right)$. 


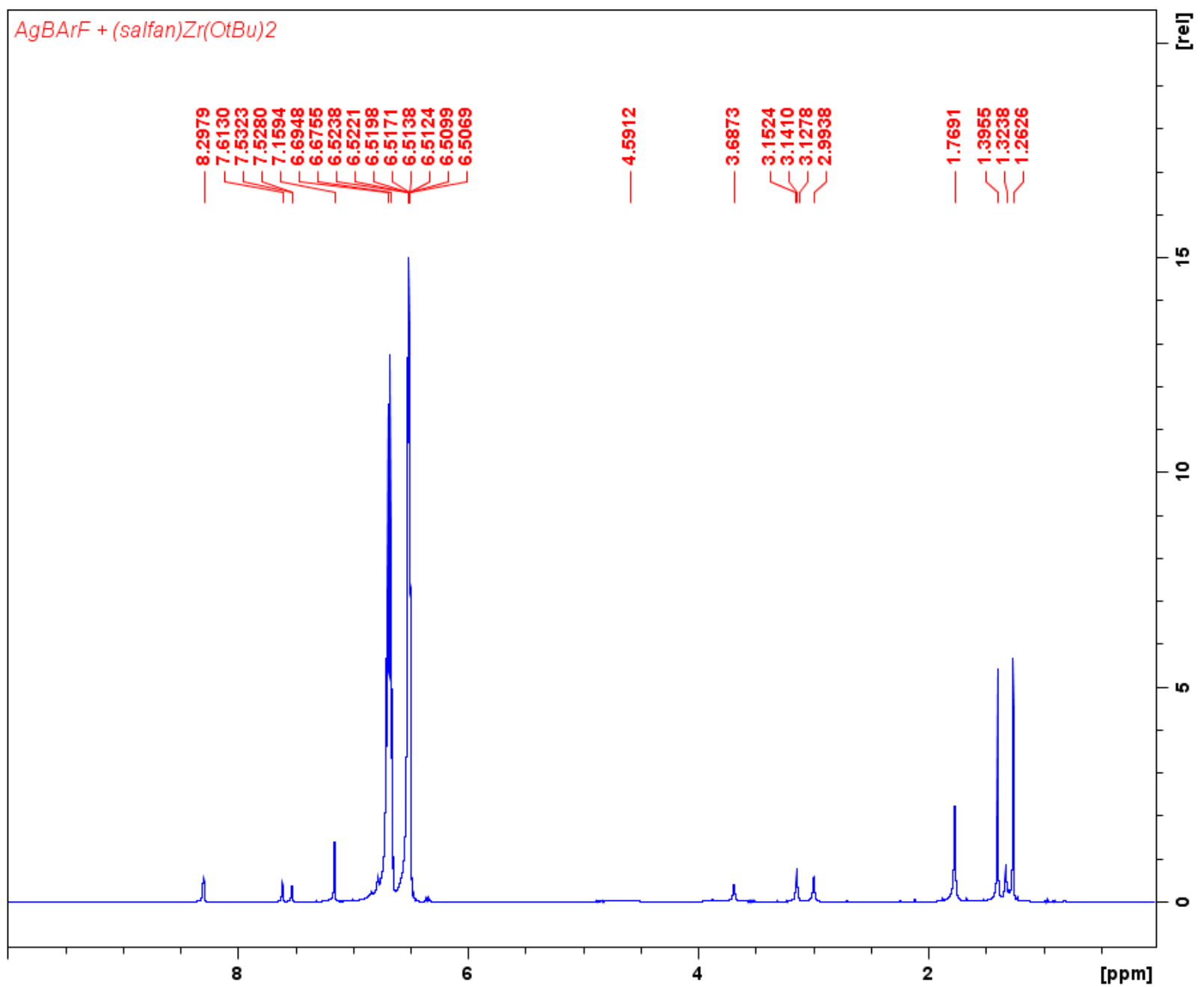

Figure S46. Table 3, entry 7. Oxidation of (salfan) $\mathrm{Zr}\left(\mathrm{O}^{t} \mathrm{Bu}\right)_{2}$ by $\mathrm{Ag}\left[\mathrm{BAr}{ }^{\mathrm{F}} 4\right] .{ }^{1} \mathrm{H}$ NMR $(300 \mathrm{MHz}$, $\left.25{ }^{\circ} \mathrm{C}, \mathrm{C}_{6} \mathrm{D}_{6}\right), \delta(\mathrm{ppm}): 8.30(\mathrm{~s}, 2 \mathrm{H},-\mathrm{OH}), 7.61(\mathrm{~d}, 2 \mathrm{H}, \mathrm{PhH}), 7.53(\mathrm{~d}, 2 \mathrm{H}, \mathrm{PhH}), 6.69(\mathrm{CFCH}$ 1,2-difluorobenzene), 6.52 (CFCHCH 1,2-difluorobenzene), 4.59 (br, 2H, CpH), 3.69 (t, 4H, $\mathrm{CpH}), 3.14$ (s, 8H, CpH), 2.99 (s, 6H, NCH 2$), 1.77$ (s, 18H, NCH 3$), 1.40$ (s, 18H, C(CH3)3), 1.26 $\left(\mathrm{s}, 18 \mathrm{H}, \mathrm{C}\left(\mathrm{CH}_{3}\right)_{3}\right)$. 


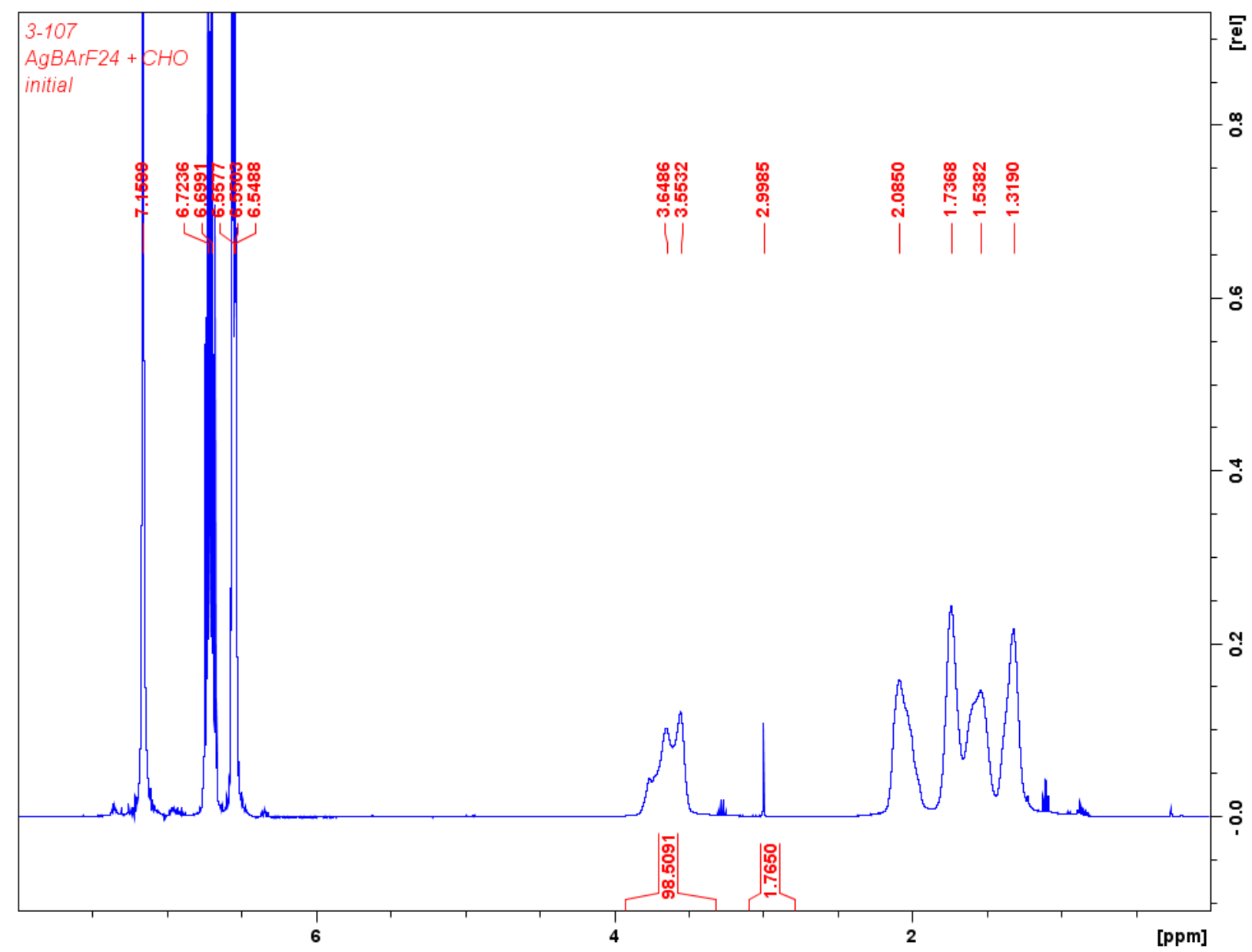

Figure S47. Table 3, entry 8. Polymerization of 100 equivalents of cyclohexene oxide by $\mathrm{AgB}\left(\mathrm{C}_{6} \mathrm{~F}_{5}\right)_{4}$ at ambient temperature. ${ }^{1} \mathrm{H}$ NMR $\left(300 \mathrm{MHz}, 25{ }^{\circ} \mathrm{C}, \mathrm{C}_{6} \mathrm{D}_{6}\right), \delta(\mathrm{ppm}): 6.71(\mathrm{CFCH}$ 1,2-difluorobenzene), 6.55 (CFCHCH 1,2-difluorobenzene), 3.60 (m, 2H, COCH PCHO), 3.00 (s, 2H, $\left.\mathrm{COCH}_{2} \mathrm{CHO}\right), 2.08\left(\mathrm{~m}, 2 \mathrm{H}, \mathrm{COCHCH}_{2} \mathrm{PCHO}\right), 1.74\left(\mathrm{~m}, 2 \mathrm{H}, \mathrm{COCHCH}_{2} \mathrm{PCHO}\right), 1.54$ (m, 2H, $\left.\mathrm{COCHCH}_{2} \mathrm{CH}_{2} \mathrm{PCHO}\right), 1.31$ (m, 2H, $\mathrm{COCHCH}_{2} \mathrm{CH}_{2} \mathrm{PCHO}$ ). 


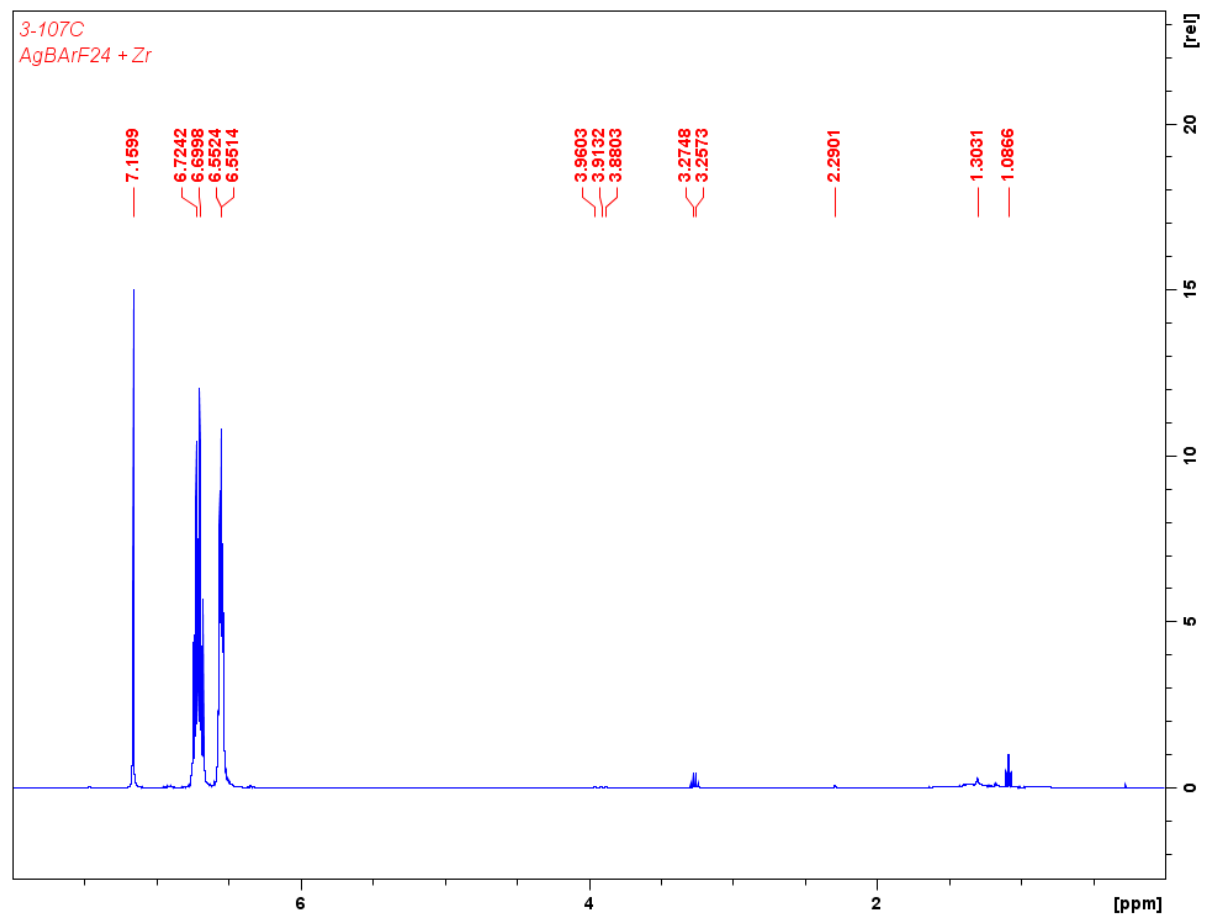

Figure S48. Table 3, entry 8. ${ }^{1} \mathrm{H}$ NMR $\left(300 \mathrm{MHz}, 25{ }^{\circ} \mathrm{C}, \mathrm{C}_{6} \mathrm{D}_{6}\right)$ spectrum of the oxidation of (salfan) $\mathrm{Zr}\left(\mathrm{O}^{t} \mathrm{Bu}\right)_{2}$ by $\mathrm{AgB}\left(\mathrm{C}_{6} \mathrm{~F}_{5}\right)_{4}$.

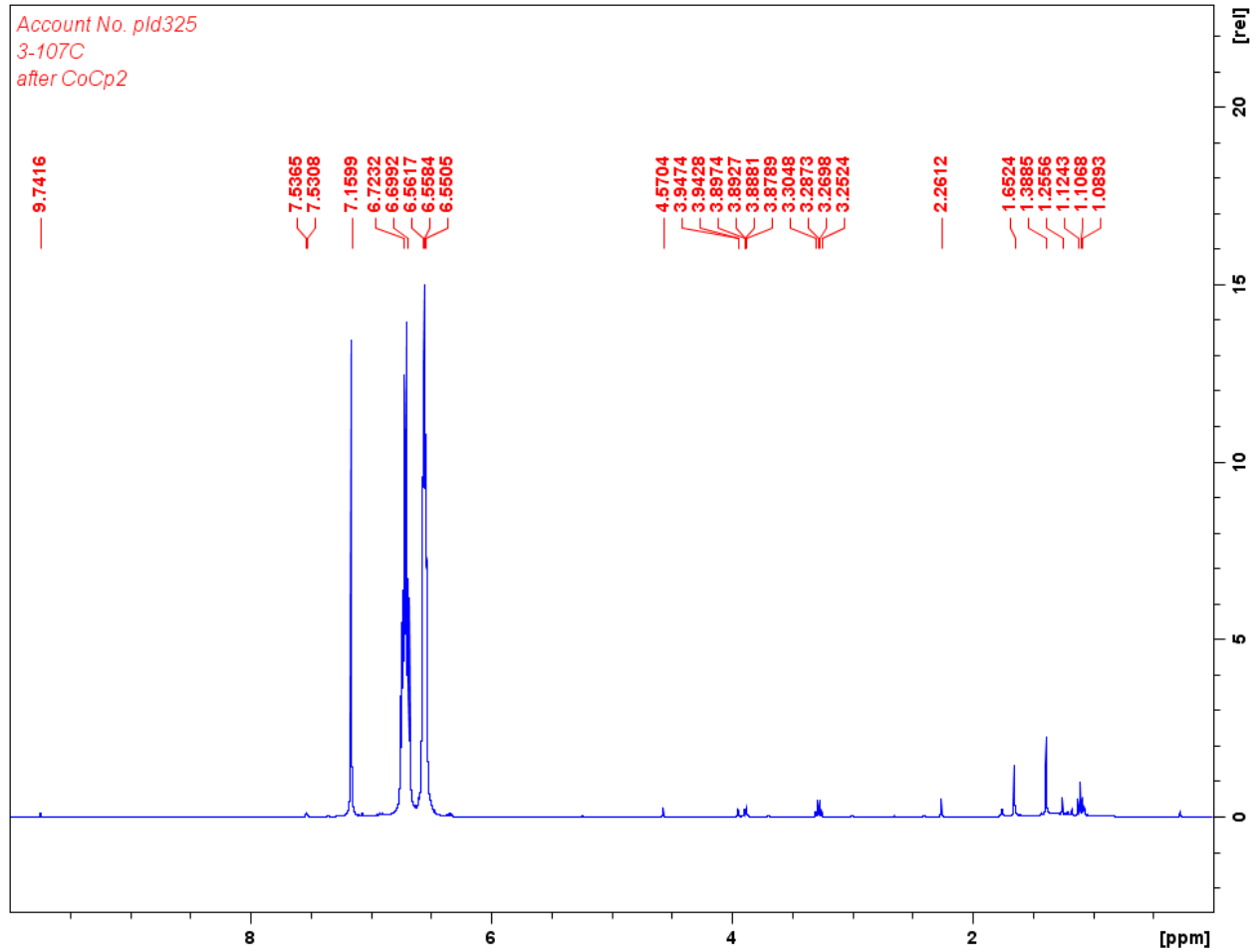

Figure S49. Table 3, entry 8. Oxidation of (salfan) $\mathrm{Zr}\left(\mathrm{O}^{t} \mathrm{Bu}\right)_{2}$ by $\mathrm{AgB}\left(\mathrm{C}_{6} \mathrm{~F}_{5}\right)_{4}$ and subsequent reduction by $\mathrm{CoCp}_{2} .{ }^{1} \mathrm{H}$ NMR $\left(300 \mathrm{MHz}, 25{ }^{\circ} \mathrm{C}, \mathrm{C}_{6} \mathrm{D}_{6}\right), \delta(\mathrm{ppm}): 9.74(\mathrm{~s}, 2 \mathrm{H},-\mathrm{OH}), 7.53(\mathrm{~d}, J=$ $2.3 \mathrm{~Hz}, 2 \mathrm{H}, \mathrm{PhH}), 6.69$ (CFCH 1,2-difluorobenzene), 6.55 (CFCHCH 1,2-difluorobenzene), 3.91 $(\mathrm{t}, \mathrm{J}=1.8 \mathrm{~Hz}, 4 \mathrm{H}, \mathrm{CpH}), 3.86\left(\mathrm{br}, 8 \mathrm{H}\right.$, overlapping $\mathrm{CpH}$ and $\left.\mathrm{NCH}_{2}\right), 2.26\left(\mathrm{~s}, 4 \mathrm{H}, \mathrm{NCH}_{3}\right), 1.65(\mathrm{~s}$, $\left.18 \mathrm{H}, \mathrm{C}\left(\mathrm{CH}_{3}\right)_{3}\right), 1.38\left(\mathrm{~s}, 18 \mathrm{H}, \mathrm{C}\left(\mathrm{CH}_{3}\right)_{3}\right)$. 


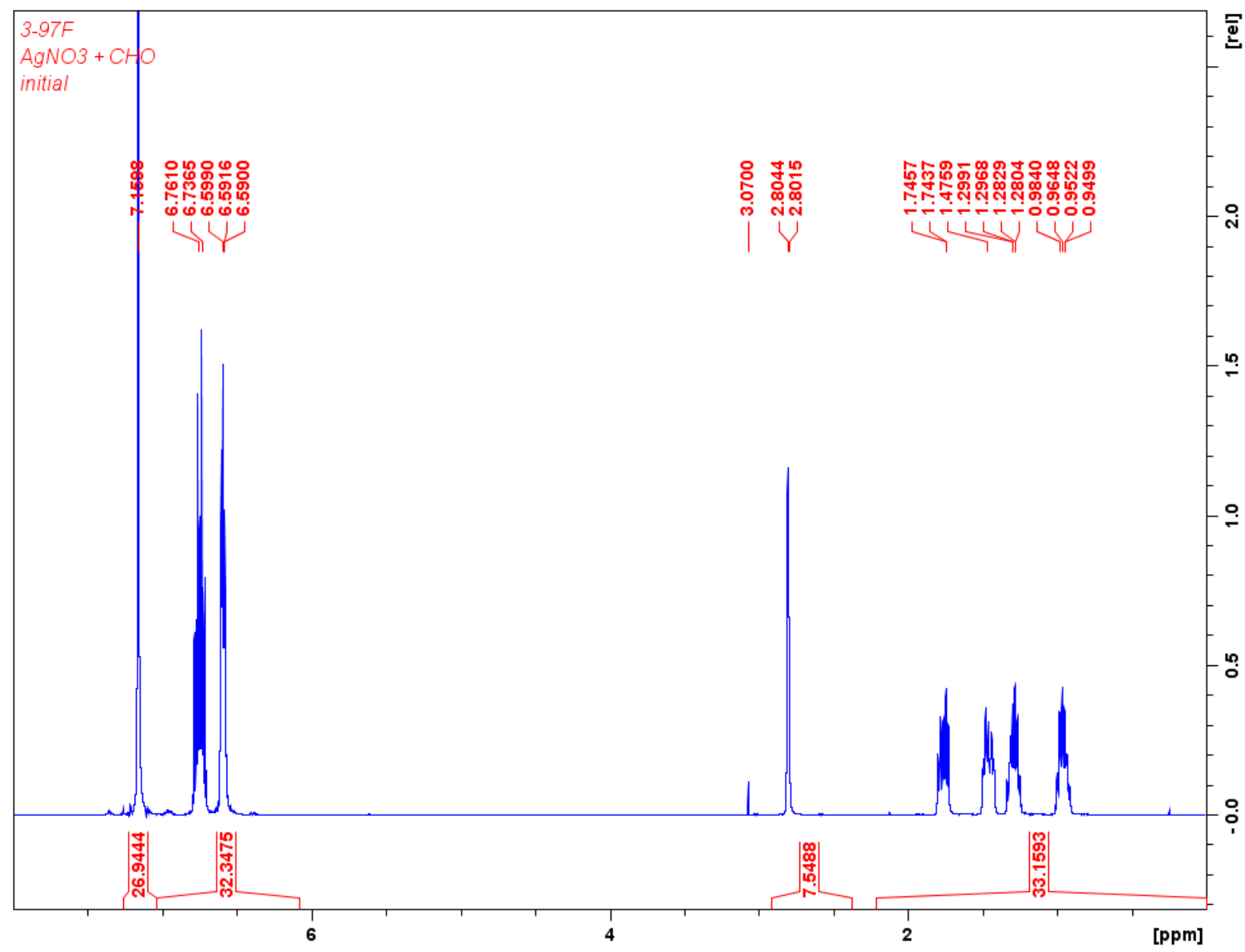

Figure S50. Table 3, entry 9. Polymerization of 100 equivalents of cyclohexene oxide by $\mathrm{AgNO}_{3}$ at ambient temperature. ${ }^{1} \mathrm{H} \mathrm{NMR}\left(300 \mathrm{MHz}, 25{ }^{\circ} \mathrm{C}, \mathrm{C}_{6} \mathrm{D}_{6}\right), \delta(\mathrm{ppm}): 6.74(\mathrm{CFCH} 1,2-$ difluorobenzene), 6.59 (CFCHCH 1,2-difluorobenzene), 2.80 (s, 2H, $\mathrm{COCH}_{2} \mathrm{CHO}$ ), 1.75 (m, $\left.2 \mathrm{H}, \mathrm{COCHCH}_{2} \mathrm{CHO}\right), 1.48\left(\mathrm{~m}, 2 \mathrm{H}, \mathrm{COCHCH}_{2} \mathrm{CHO}\right), 1.29\left(\mathrm{~m}, 2 \mathrm{H}, \mathrm{COCHCH}_{2} \mathrm{CH}_{2} \mathrm{CHO}\right), 0.96$ (m, $\left.2 \mathrm{H}, \mathrm{COCHCH}_{2} \mathrm{CH}_{2} \mathrm{CHO}\right)$. 


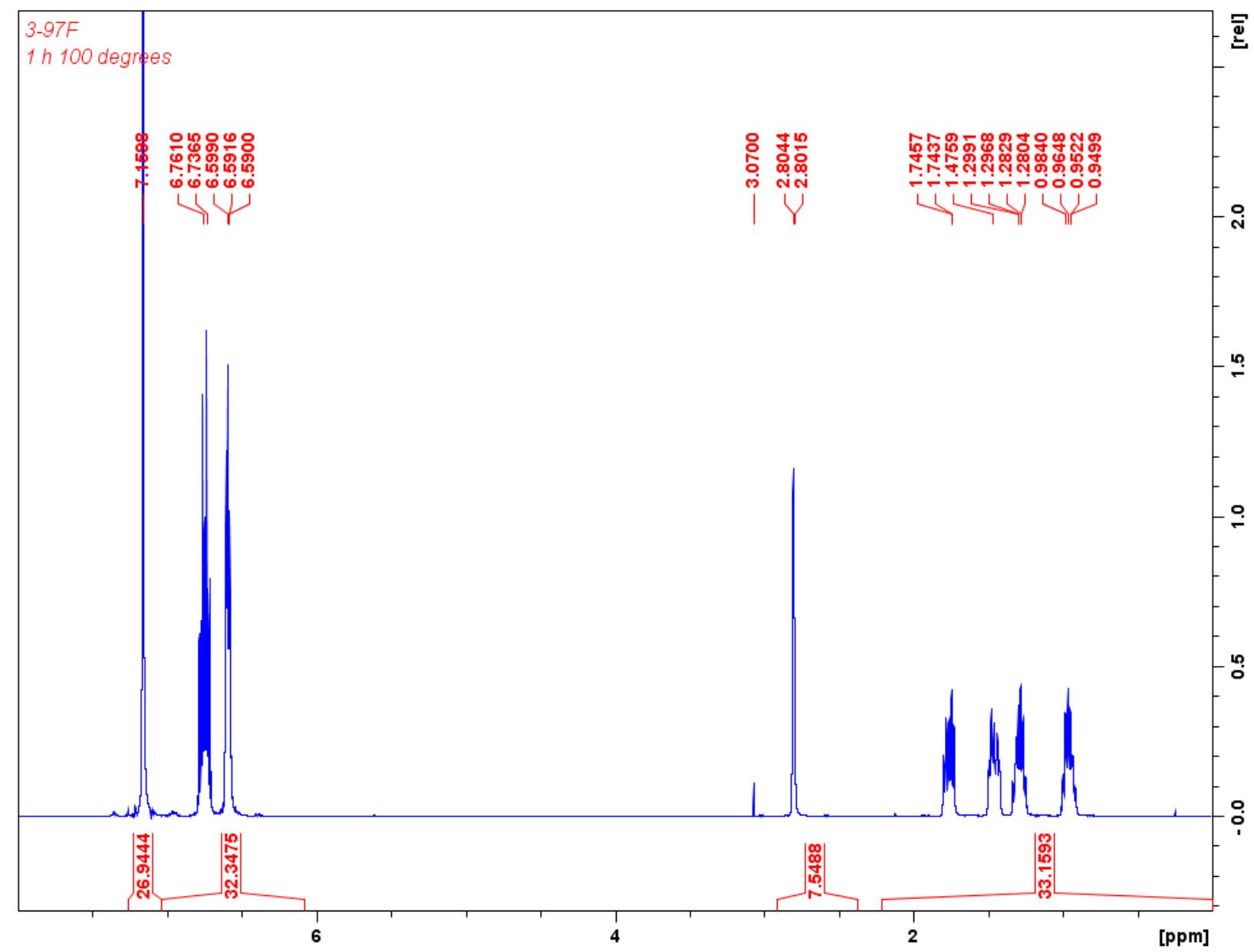

Figure S51. Table 3, entry 9. Polymerization of 100 equivalents of cyclohexene oxide by $\mathrm{AgNO}_{3}$ after 1 hour at $100{ }^{\circ} \mathrm{C} .{ }^{1} \mathrm{H}$ NMR $\left(300 \mathrm{MHz}, 25{ }^{\circ} \mathrm{C}, \mathrm{C}_{6} \mathrm{D}_{6}\right), \delta(\mathrm{ppm}): 6.76(\mathrm{CFCH} 1,2-$ difluorobenzene), 6.59 (CFCHCH 1,2-difluorobenzene), 2.80 (s, 2H, $\left.\mathrm{COCH}_{2} \mathrm{CHO}\right), 1.74$ (m, $\left.2 \mathrm{H}, \mathrm{COCHCH}_{2} \mathrm{CHO}\right), 1.47\left(\mathrm{~m}, 2 \mathrm{H}, \mathrm{COCHCH}_{2} \mathrm{CHO}\right), 1.28\left(\mathrm{~m}, 2 \mathrm{H}, \mathrm{COCHCH}_{2} \mathrm{CH}_{2} \mathrm{CHO}\right), 0.96$ (m, $\left.2 \mathrm{H}, \mathrm{COCHCH}_{2} \mathrm{CH}_{2} \mathrm{CHO}\right)$. 


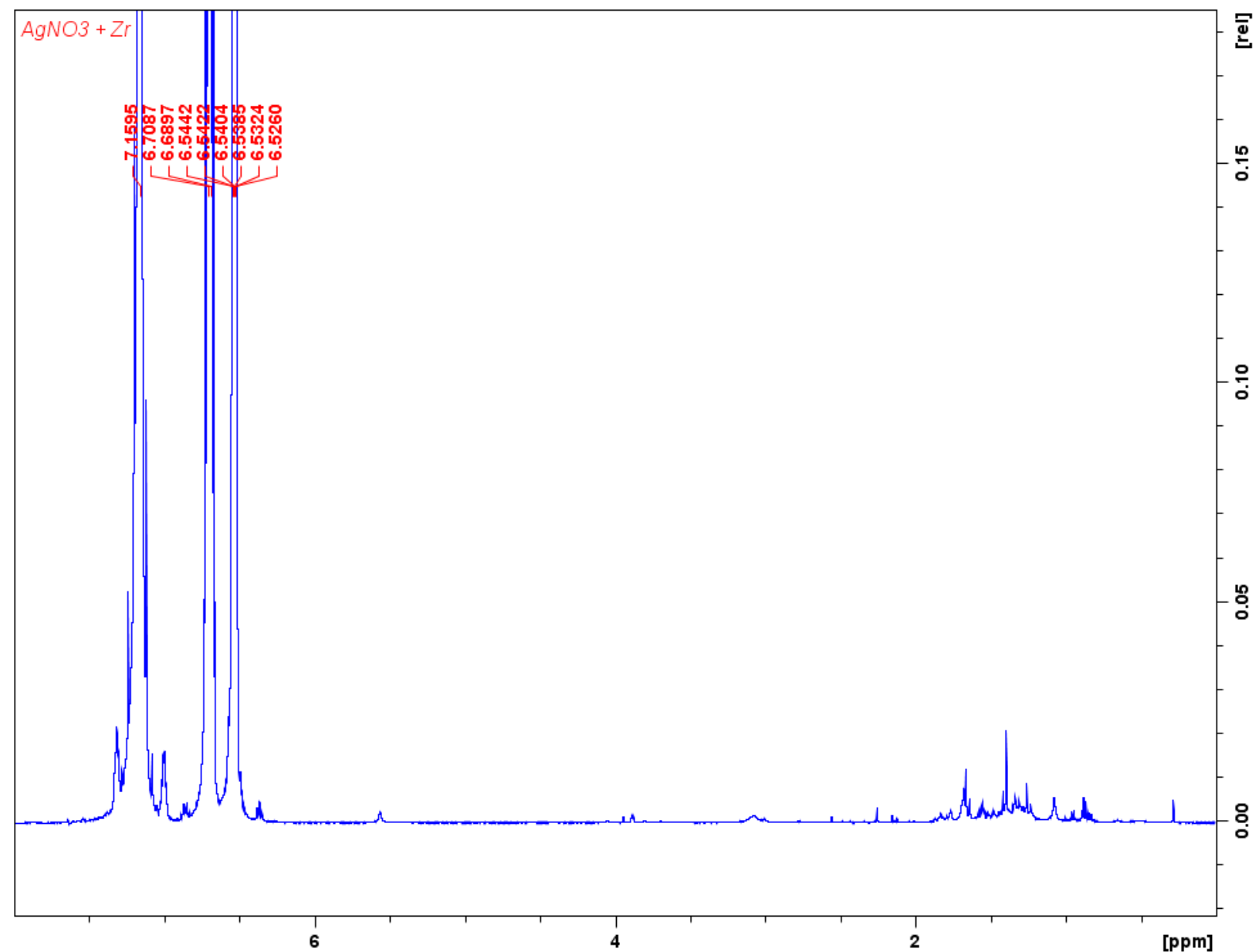

Figure S52. Table 3, entry 9. ${ }^{1} \mathrm{H}$ NMR $\left(300 \mathrm{MHz}, 25{ }^{\circ} \mathrm{C}, \mathrm{C}_{6} \mathrm{D}_{6}\right)$ spectrum of the oxidation of (salfan) $\mathrm{Zr}\left(\mathrm{O}^{t} \mathrm{Bu}\right)_{2}$ by $\mathrm{AgNO}_{3}$. 


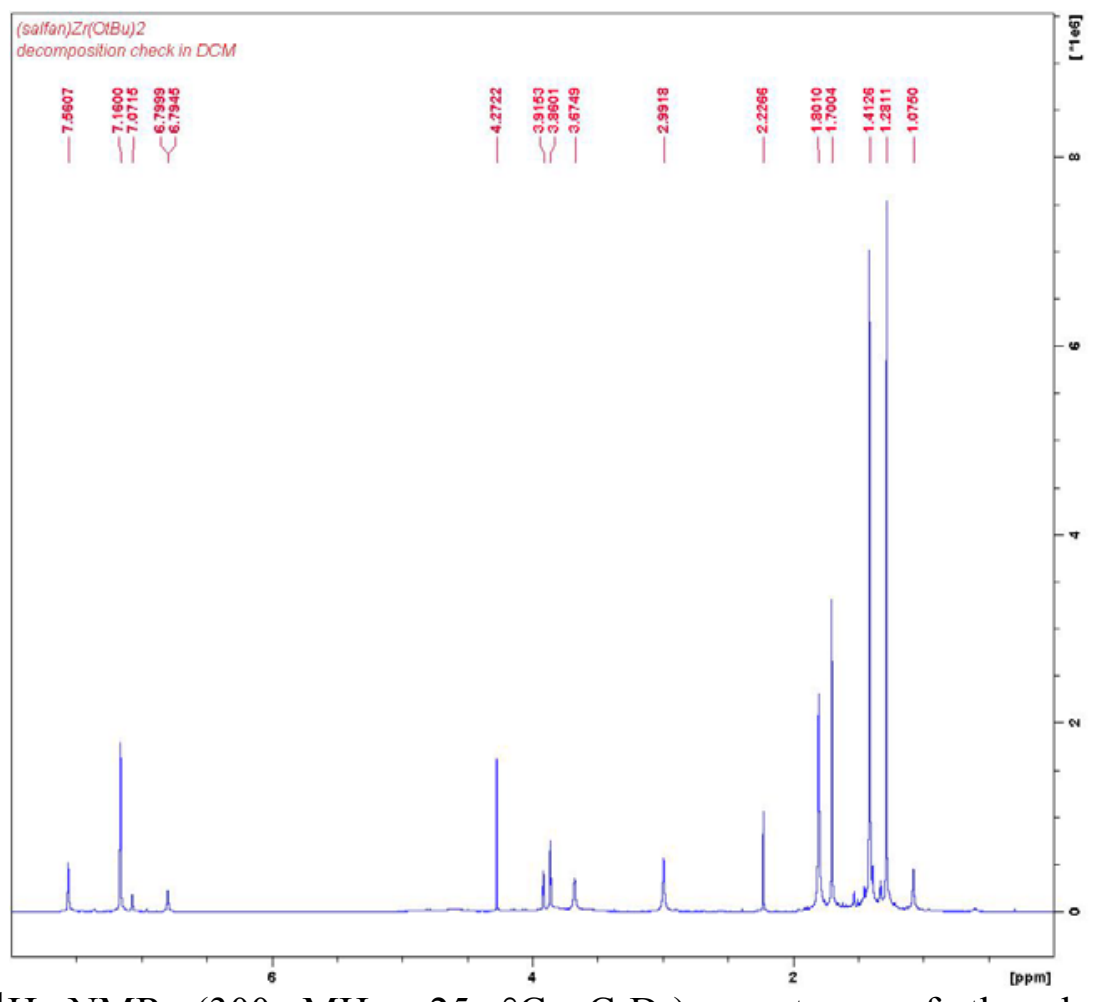

Figure S53. ${ }^{1} \mathrm{H}$ NMR $\left(300 \mathrm{MHz}, 25{ }^{\circ} \mathrm{C}, \mathrm{C}_{6} \mathrm{D}_{6}\right)$ spectrum of the decomposition of (salfan) $\mathrm{Zr}\left(\mathrm{O}^{\mathrm{t}} \mathrm{Bu}\right)_{2}$ in $\mathrm{CH}_{2} \mathrm{Cl}_{2}$.

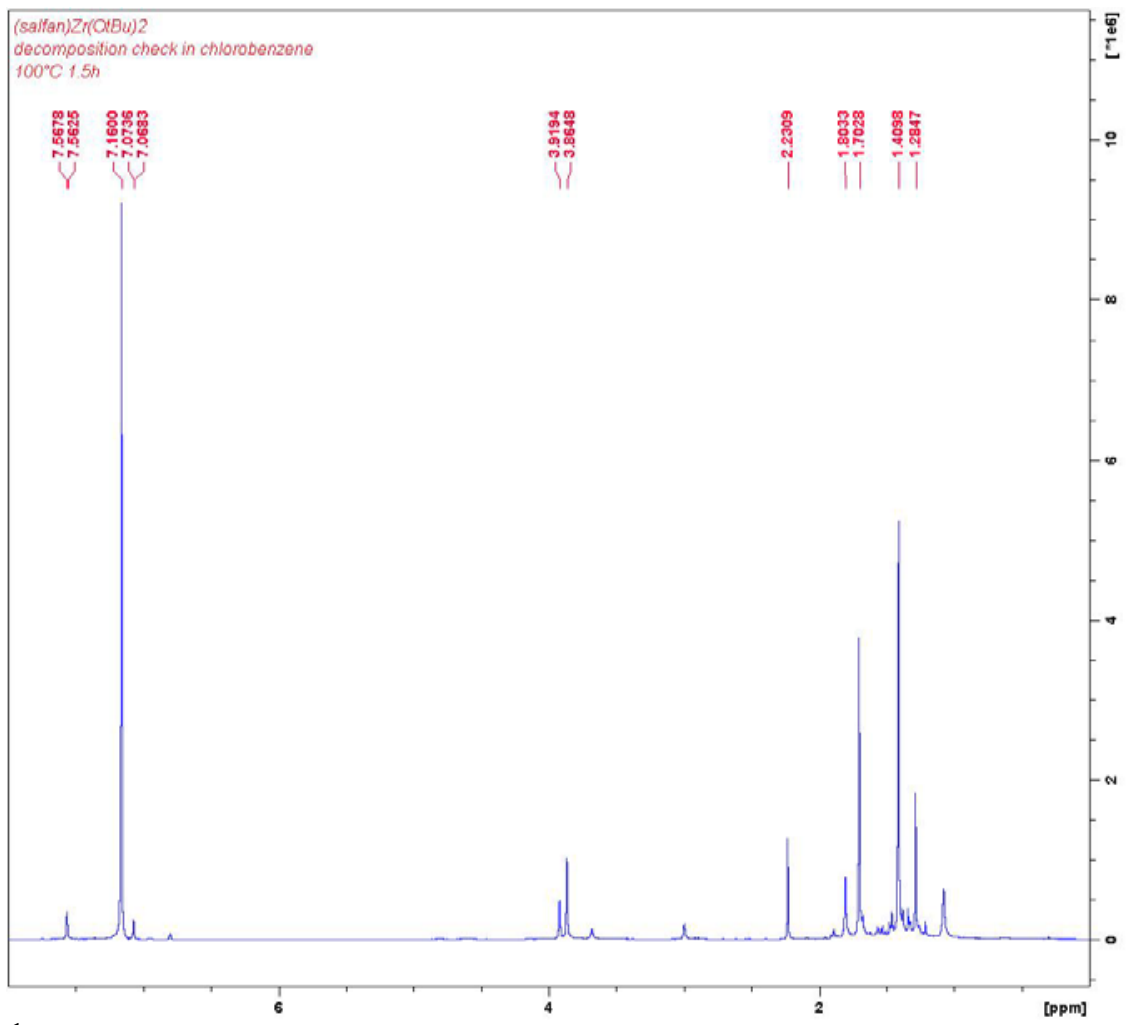

Figure S54. ${ }^{1} \mathrm{H}$ NMR (300 $\left.\mathrm{MHz}, 25{ }^{\circ} \mathrm{C}, \mathrm{C}_{6} \mathrm{D}_{6}\right)$ spectrum of the decomposition of (salfan) $\mathrm{Zr}\left(\mathrm{O}^{\mathrm{t}} \mathrm{Bu}\right)_{2}$ in chlorobenzene after 1.5 hours at $100{ }^{\circ} \mathrm{C}$. 


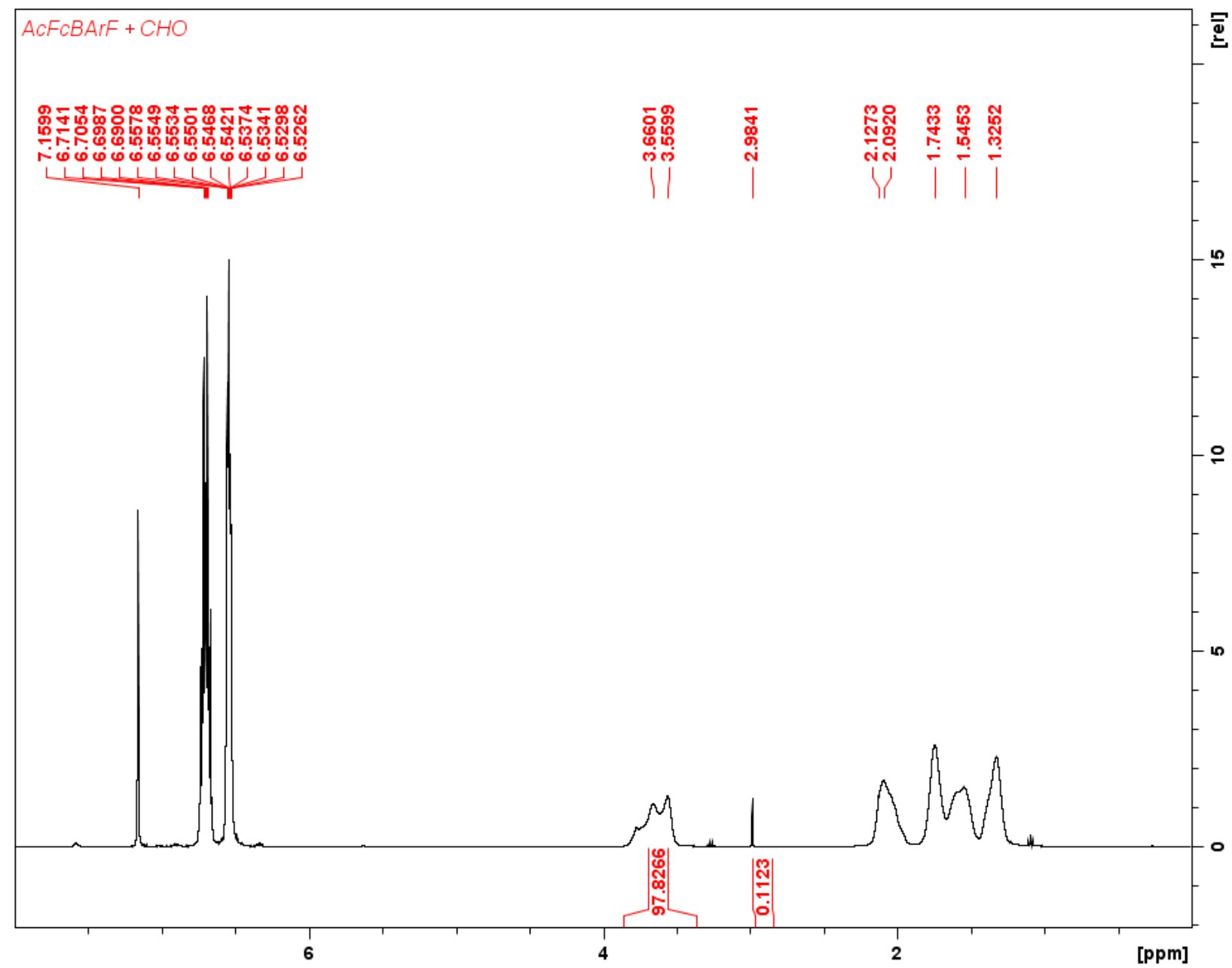

Figure S55. Polymerization of cyclohexene oxide by ${ }^{\mathrm{Ac}} \mathrm{FcBAr}{ }^{\mathrm{F}} .{ }^{1} \mathrm{H} \mathrm{NMR}\left(300 \mathrm{MHz}, 25{ }^{\circ} \mathrm{C}\right.$, $\mathrm{C}_{6} \mathrm{D}_{6}$ ), $\delta$ (ppm): 6.70 (CFCH 1,2-difluorobenzene), 6.55 (CFCHCH 1,2-difluorobenzene), 3.62 $(\mathrm{m}, 2 \mathrm{H}, \mathrm{COCH} \mathrm{PCHO}), 2.98\left(\mathrm{~s}, 2 \mathrm{H}, \mathrm{COCH}_{2} \mathrm{CHO}\right), 2.10\left(\mathrm{~m}, 2 \mathrm{H}, \mathrm{COCHCH}_{2} \mathrm{PCHO}\right), 1.74(\mathrm{~m}$, $\left.2 \mathrm{H}, \mathrm{COCHCH}_{2} \mathrm{PCHO}\right), 1.54\left(\mathrm{~m}, 2 \mathrm{H}, \mathrm{COCHCH}_{2} \mathrm{CH}_{2} \mathrm{PCHO}\right), 1.33\left(\mathrm{~m}, 2 \mathrm{H}, \mathrm{COCHCH}_{2} \mathrm{CH}_{2}\right.$ $\mathrm{PCHO})$. 


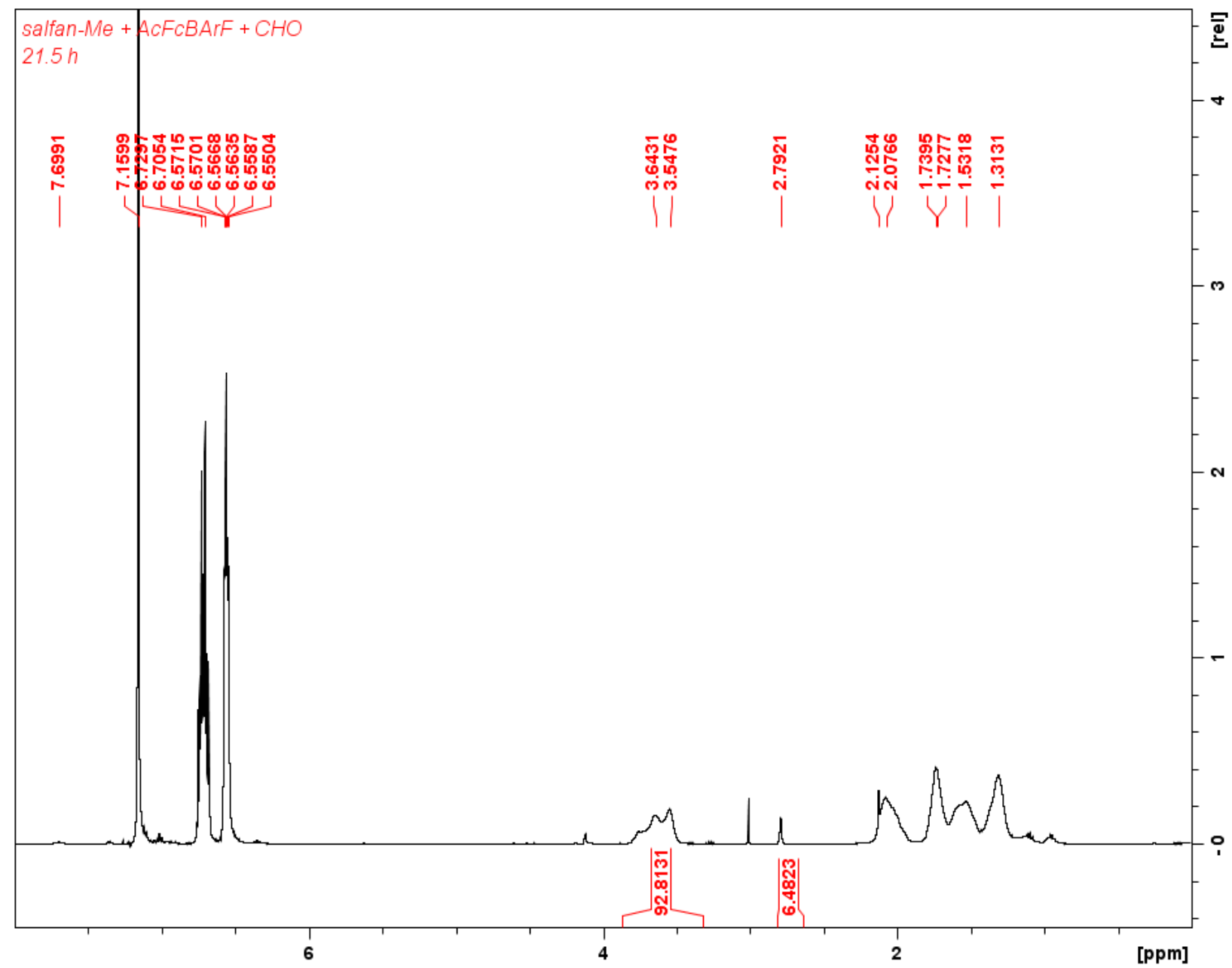

Figure S56. Polymerization of cyclohexene oxide by [salfan] $\left[\mathrm{BAr}^{\mathrm{F}}\right] .{ }^{1} \mathrm{H}$ NMR $\left(300 \mathrm{MHz}, 25{ }^{\circ} \mathrm{C}\right.$, $\mathrm{C}_{6} \mathrm{D}_{6}$ ), $\delta$ (ppm): 6.71 ( $\mathrm{CFCH}$ 1,2-difluorobenzene), 6.56 (CFCHCH 1,2-difluorobenzene), 3.59 (m, 2H, COCH PCHO), 2.80 (s, 2H, $\left.\mathrm{COCH}_{2} \mathrm{CHO}\right), 2.07$ (m, 2H, $\left.\mathrm{COCHCH}_{2} \mathrm{PCHO}\right), 1.73$ (m, $\left.2 \mathrm{H}, \mathrm{COCHCH}_{2} \mathrm{PCHO}\right), 1.53\left(\mathrm{~m}, 2 \mathrm{H}, \mathrm{COCHCH}_{2} \mathrm{CH}_{2} \mathrm{PCHO}\right), 1.31\left(\mathrm{~m}, 2 \mathrm{H}, \mathrm{COCHCH}_{2} \mathrm{CH}_{2}\right.$ $\mathrm{PCHO})$. 


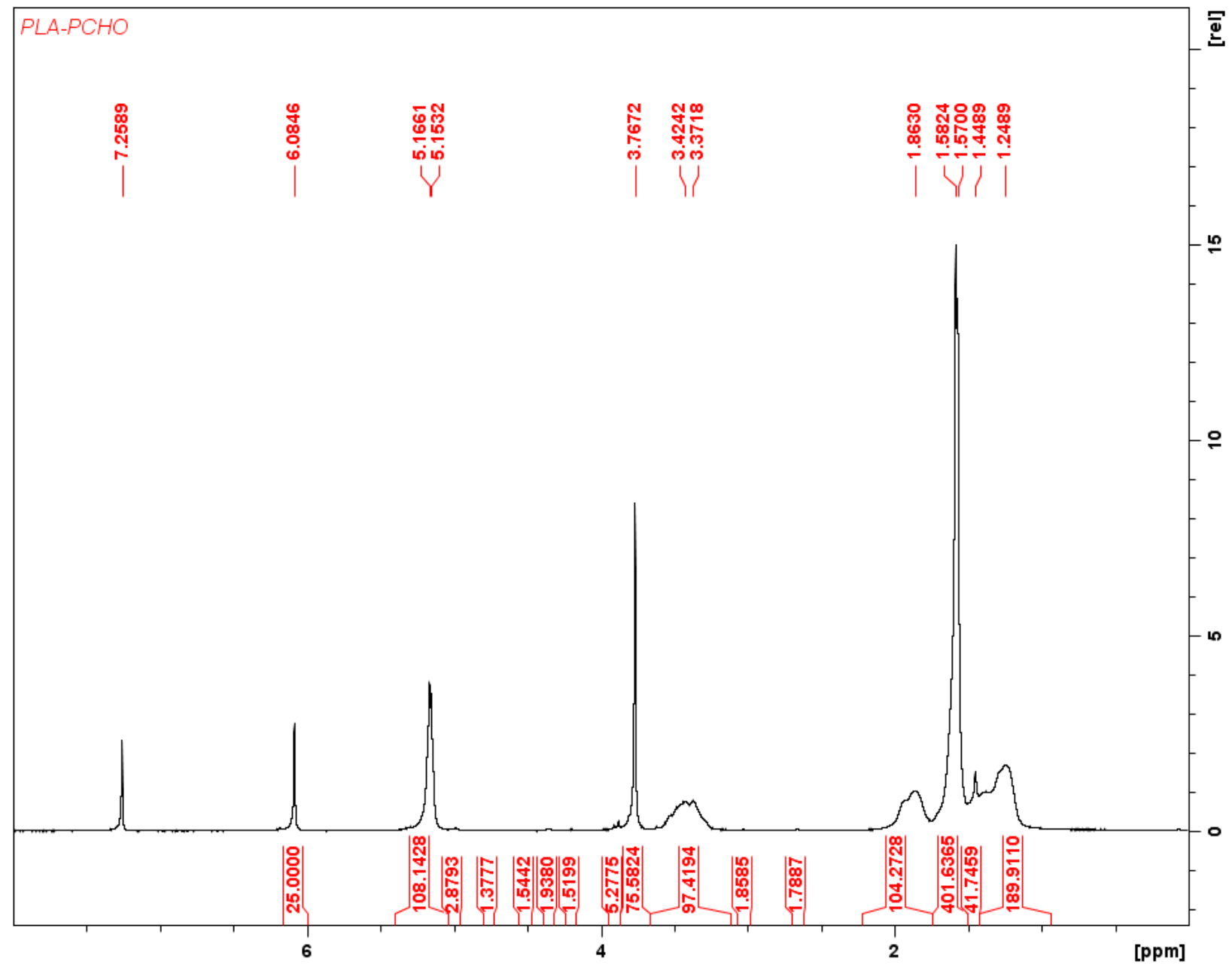

Figure S57. Table 4, entry 1. Table S2, entry 1. Polymerization of 100 equivalents of L-lactide, and cyclohexene oxide, monomers added sequentially, using catalyst redox switch "red-ox". ${ }^{1} \mathrm{H}$ NMR (300 MHz, $\left.25^{\circ} \mathrm{C}, \mathrm{CDCl}_{3}\right), \delta$ (ppm): 6.08 (s, 3H, PhH TMB), 5.16 (q, 2H, CHCH3 PLA), 3.77 (s, 9H, $\left.\mathrm{CH}_{3} \mathrm{TMB}\right), 3.40$ (m, 2H, COCH PCHO), 1.86 (m, 2H, COCHCH $\mathrm{PCHO}_{2}, 1.57$ (d, $\left.6 \mathrm{H}, \mathrm{CHCH}_{3} \mathrm{PLA}\right), 1.25$ (m, $2 \mathrm{H}, \mathrm{COCHCH}_{2} \mathrm{CH}_{2} \mathrm{PCHO}$ ). Small peaks that may be related to the end group or junctions were integrated. 


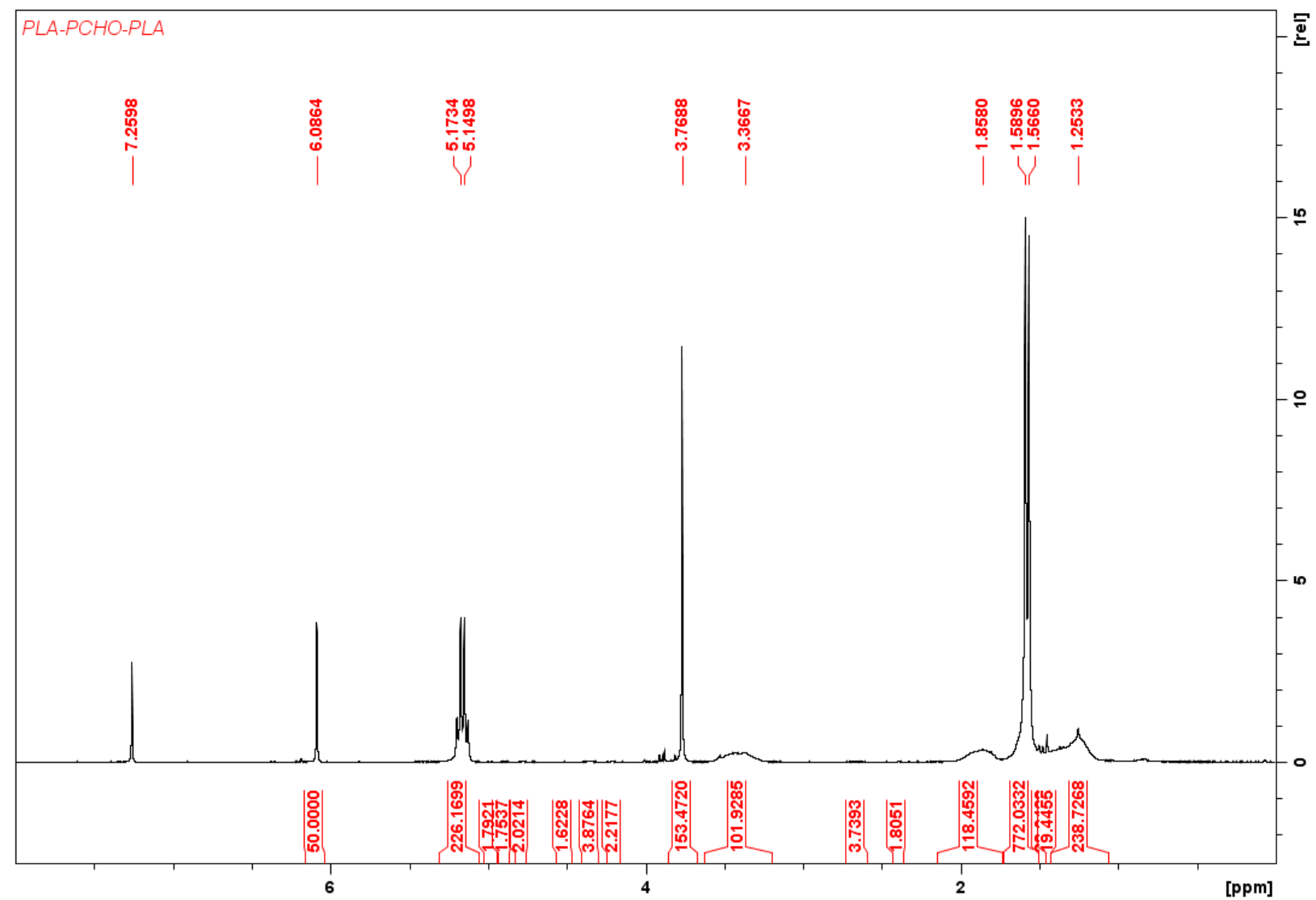

Figure S58. Table 4, entry 2. Table S3, entry 1. Polymerization of 100 equivalents of L-lactide, cyclohexene oxide and L-lactide monomers added sequentially, using catalyst redox switch "redox-red". ${ }^{1} \mathrm{H}$ NMR $\left(300 \mathrm{MHz}, 25{ }^{\circ} \mathrm{C}, \mathrm{CDCl}_{3}\right), \delta(\mathrm{ppm}): 6.08$ (s, 3H, PhH TMB), 5.16 (q, 2H, $\mathrm{CHCH}_{3} \mathrm{PLA}$ ), 3.77 (s, 9H, $\left.\mathrm{CH}_{3} \mathrm{TMB}\right), 3.37$ (m, 2H, COCH PCHO), 1.86 (m, 2H, $\mathrm{COCHCH}_{2}$ $\mathrm{PCHO}), 1.57$ (d, $\left.6 \mathrm{H}, \mathrm{CHCH}_{3} \mathrm{PLA}\right), 1.25$ (m, 2H, $\mathrm{COCHCH}_{2} \mathrm{CH}_{2} \mathrm{PCHO}$ ). Small peaks that may be related to the end group or junctions were integrated. 


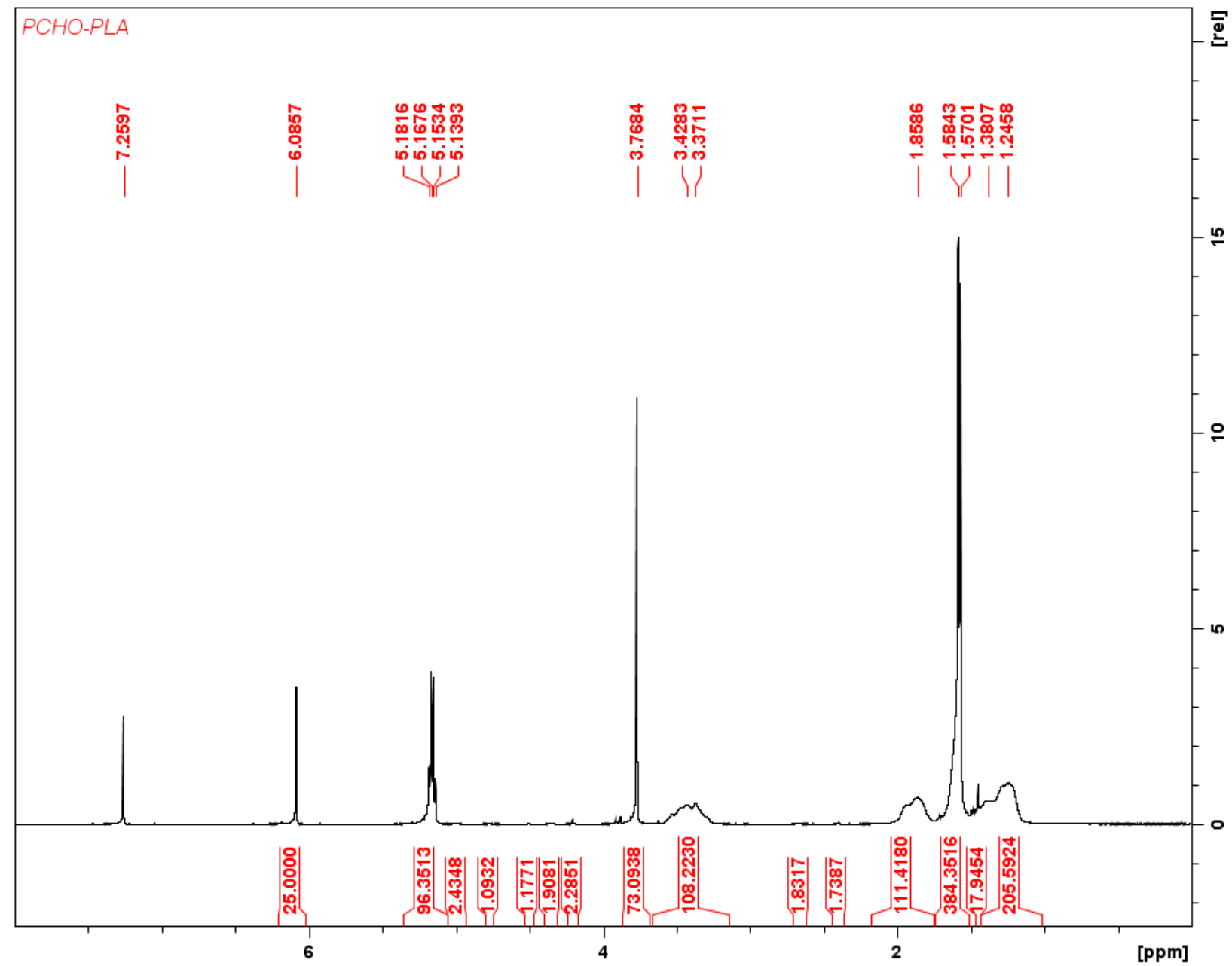

Figure S59. Table 4, entry 3. Table S4, entry 1. Polymerization of 100 equivalents of cyclohexene oxide and L-lactide, monomers added sequentially, using catalyst redox switch "ox$\operatorname{red}^{1} \mathrm{H}$ NMR (300 MHz, $\left.25{ }^{\circ} \mathrm{C}, \mathrm{CDCl}_{3}\right), \delta$ (ppm): 6.09 (s, 3H, $\left.\mathrm{PhH} \mathrm{TMB}\right), 5.16$ (q, 2H, $\mathrm{CHCH}_{3}$ PLA), 3.77 (s, 9H, CH3 TMB), 3.40 (m, 2H, COCH PCHO), 1.86 (m, 2H, COCHCH $\mathrm{PCHO}_{2}$, 1.57 (d, 6H, $\left.\mathrm{CHCH}_{3} \mathrm{PLA}\right), 1.38-1.25$ (m, $2 \mathrm{H}, \mathrm{COCHCH}_{2} \mathrm{CH}_{2} \mathrm{PCHO}$ ). Small peaks that may be related to the end group or junctions were integrated. 


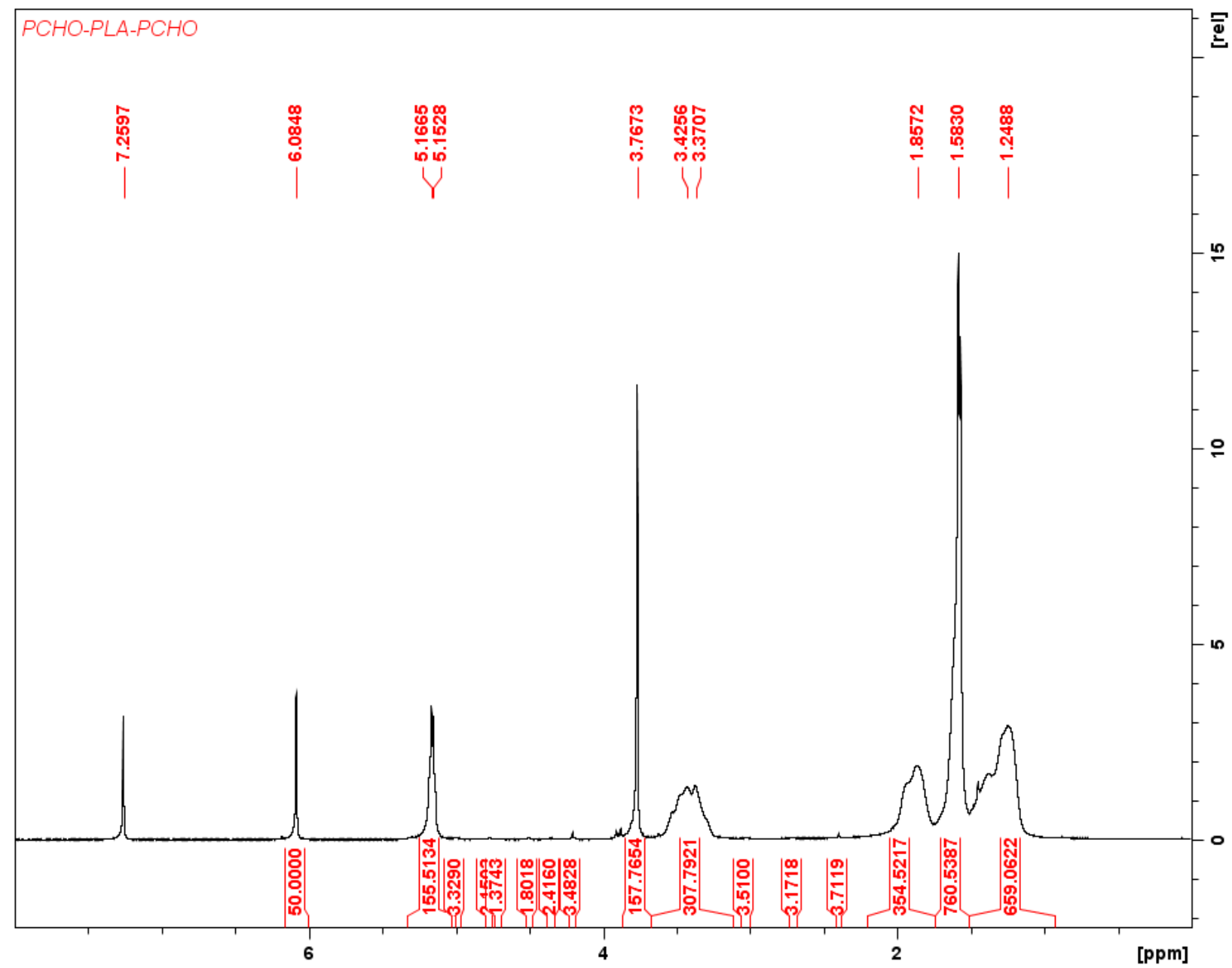

Figure S60. Table 4, entry 4. Table S5, entry 1. Polymerization of 100 equivalents of cyclohexene oxide, L-lactide, and cyclohexene oxide, monomers added sequentially, using catalyst redox switch "ox-red-ox". ${ }^{1} \mathrm{H}$ NMR $\left(300 \mathrm{MHz}, 25^{\circ} \mathrm{C}, \mathrm{CDCl}_{3}\right), \delta(\mathrm{ppm}): 6.08(\mathrm{~s}, 3 \mathrm{H}$, $\mathrm{PhH}$ TMB), 5.16 (q, 2H, $\left.\mathrm{CHCH}_{3} \mathrm{PLA}\right), 3.77$ (s, 9H, CH3 TMB), 3.40 (m, 2H, COCH PCHO), $1.86\left(\mathrm{~m}, 2 \mathrm{H}, \mathrm{COCHCH}_{2} \mathrm{PCHO}\right), 1.57$ (d, 6H, $\left.\mathrm{CHCH}_{3} \mathrm{PLA}\right), 1.25$ (m, 2H, $\mathrm{COCHCH}_{2} \mathrm{CH}_{2}$ PCHO). Small peaks that may be related to the end group or junctions were integrated. 


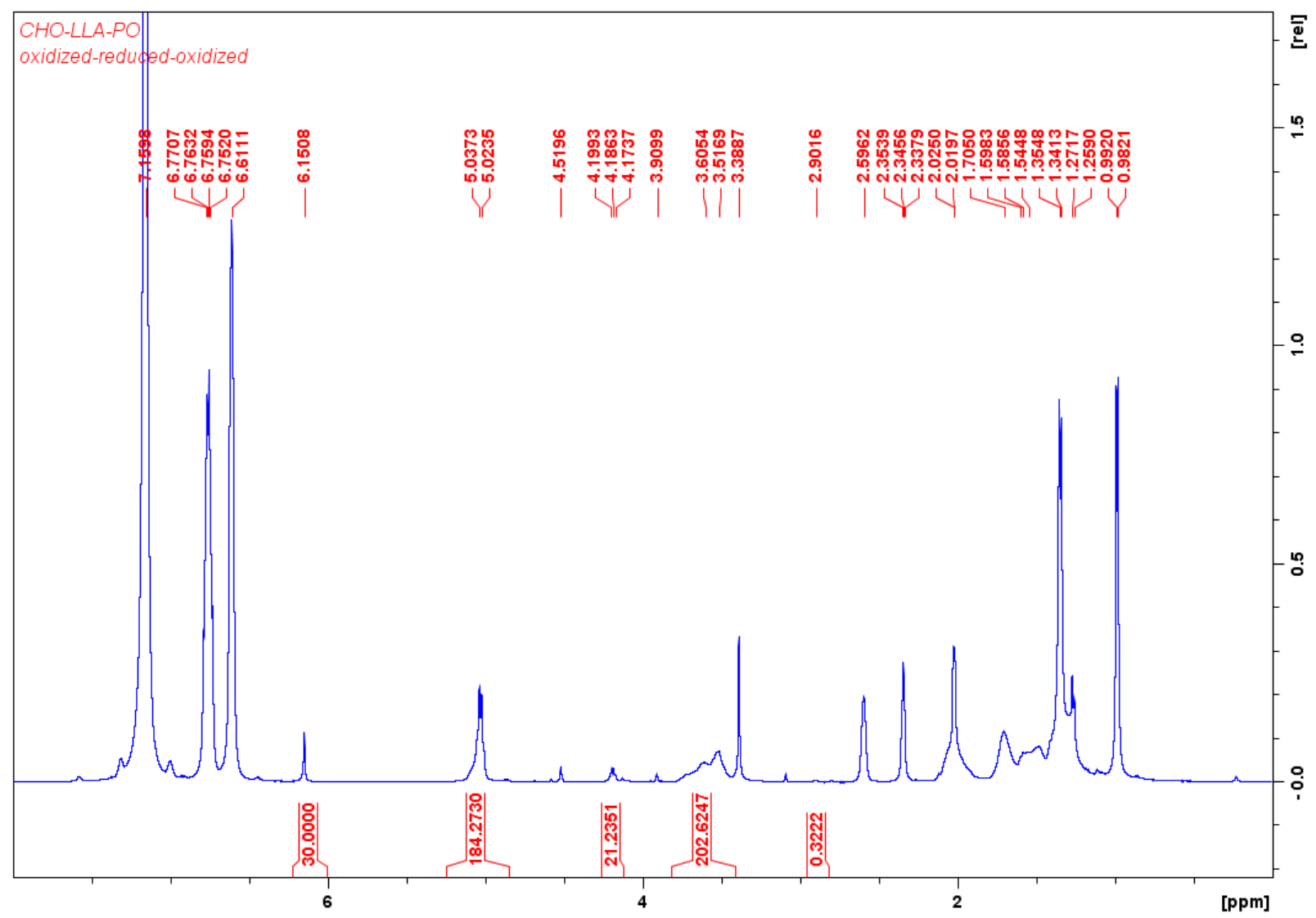

Figure S61. Table 4, entry 5. Polymerization of 100 equivalents of cyclohexene oxide, L-lactide, and propylene oxide, monomers added sequentially, using catalyst redox switch "ox-red-ox". ${ }^{1} \mathrm{H}$ NMR (300 MHz, $\left.25^{\circ} \mathrm{C}, \mathrm{C}_{6} \mathrm{D}_{6}\right), \delta$ (ppm): 6.77 (CFCH 1,2-difluorobenzene), 6.61 (CFCHCH 1,2difluorobenzene), 6.15 (s, 3H, $\mathrm{PhH} \mathrm{TMB}$ ), 5.03 (q, 2H, $\left.\mathrm{CHCH}_{3} \mathrm{PLA}\right), 4.19$ (q, 2H, CHCH3 LA), 3.55 (m, 2H, COCH PCHO), 3.39 (s, 9H, CH3 TMB), $2.90\left(\mathrm{~m}, 1 \mathrm{H}, \mathrm{COCHCH}_{3} \mathrm{PPO}\right), 2.60(\mathrm{~m}$, $\left.1 \mathrm{H}, \mathrm{COCHCH}_{3} \mathrm{PO}\right), 2.34$ (m, 1H, $\left.\mathrm{COCH}_{2} \mathrm{PO}\right), 2.02$ (m, 2H, $\left.\mathrm{COCHCH}_{2} \mathrm{PCHO}\right), 1.70-1.54$ (m, $2 \mathrm{H}, \mathrm{COCHCH}_{2} \mathrm{PCHO}$ ), 1.35 (d, 3H, $\left.\mathrm{CH}_{3} \mathrm{PLA}\right), 1.26$ (m, 2H, $\mathrm{COCHCH}_{2} \mathrm{CH}_{2} \mathrm{CHO}$ ), 0.99 (d, $\left.3 \mathrm{H}, \mathrm{COCHCH}_{3} \mathrm{PO}\right)$. 


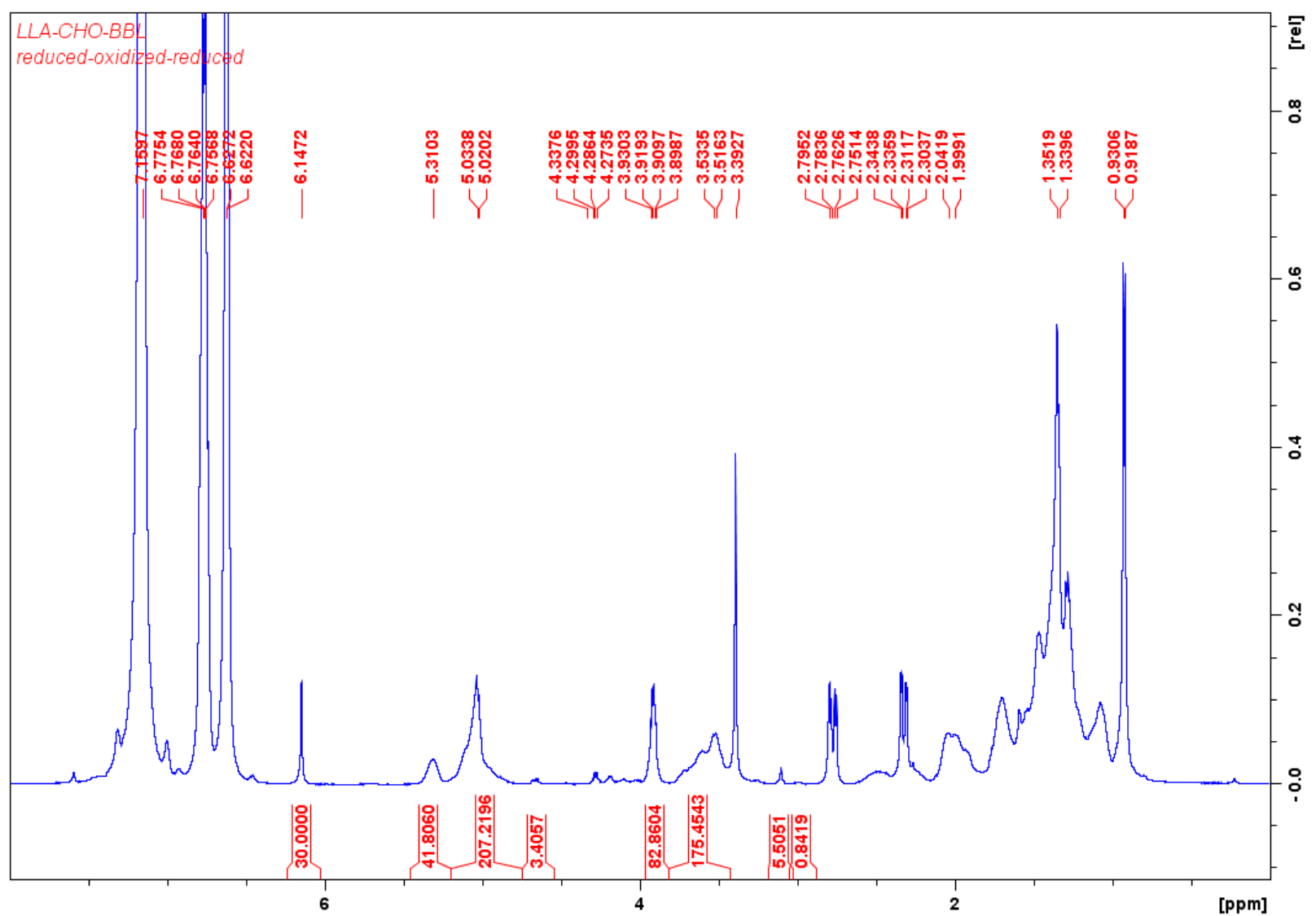

Figure S62. Table 4, entry 6. Polymerization of 100 equivalents of L-lactide, cyclohexene oxide, and $\beta$-butyrolactone, monomers added sequentially, using catalyst redox switch "red-ox-red". ${ }^{1} \mathrm{H}$ NMR (300 MHz, $\left.25^{\circ} \mathrm{C}, \mathrm{C}_{6} \mathrm{D}_{6}\right), \delta$ (ppm): 6.76 (CFCH 1,2-difluorobenzene), 6.63 (CFCHCH 1,2difluorobenzene), 6.15 (s, 3H, $\mathrm{PhH} \mathrm{TMB}), 5.31\left(\mathrm{~m}, 1 \mathrm{H}, \mathrm{OCHCH}_{3} \mathrm{PHB}\right), 5.03$ (q, 2H, $\mathrm{CHCH}_{3}$ PLA), 4.30 (m, 1H, $\left.\mathrm{OCHCH}_{3}, \mathrm{BBL}\right), 3.91$ (q, 2H, $\left.\mathrm{CHCH}_{3} \mathrm{LA}\right), 3.52$ (m, 2H, COCH PCHO), 3.39 (s, 9H, CH3 TMB), 2.77 (m, 2H, $\left.\mathrm{COCH}_{2} \mathrm{BBL}\right), 2.32\left(\mathrm{~m}, 2 \mathrm{H}, \mathrm{COCH}_{2} \mathrm{BBL}\right), 2.02$ (m, 2H, $\mathrm{COCHCH}_{2} \mathrm{PCHO}$ ), 1.34 (d, 6H, $\left.\mathrm{CHCH}_{3} \mathrm{PLA}\right), 0.92$ (t, 3H, $\mathrm{OCHCH}_{3} \mathrm{BBL}$ ). 

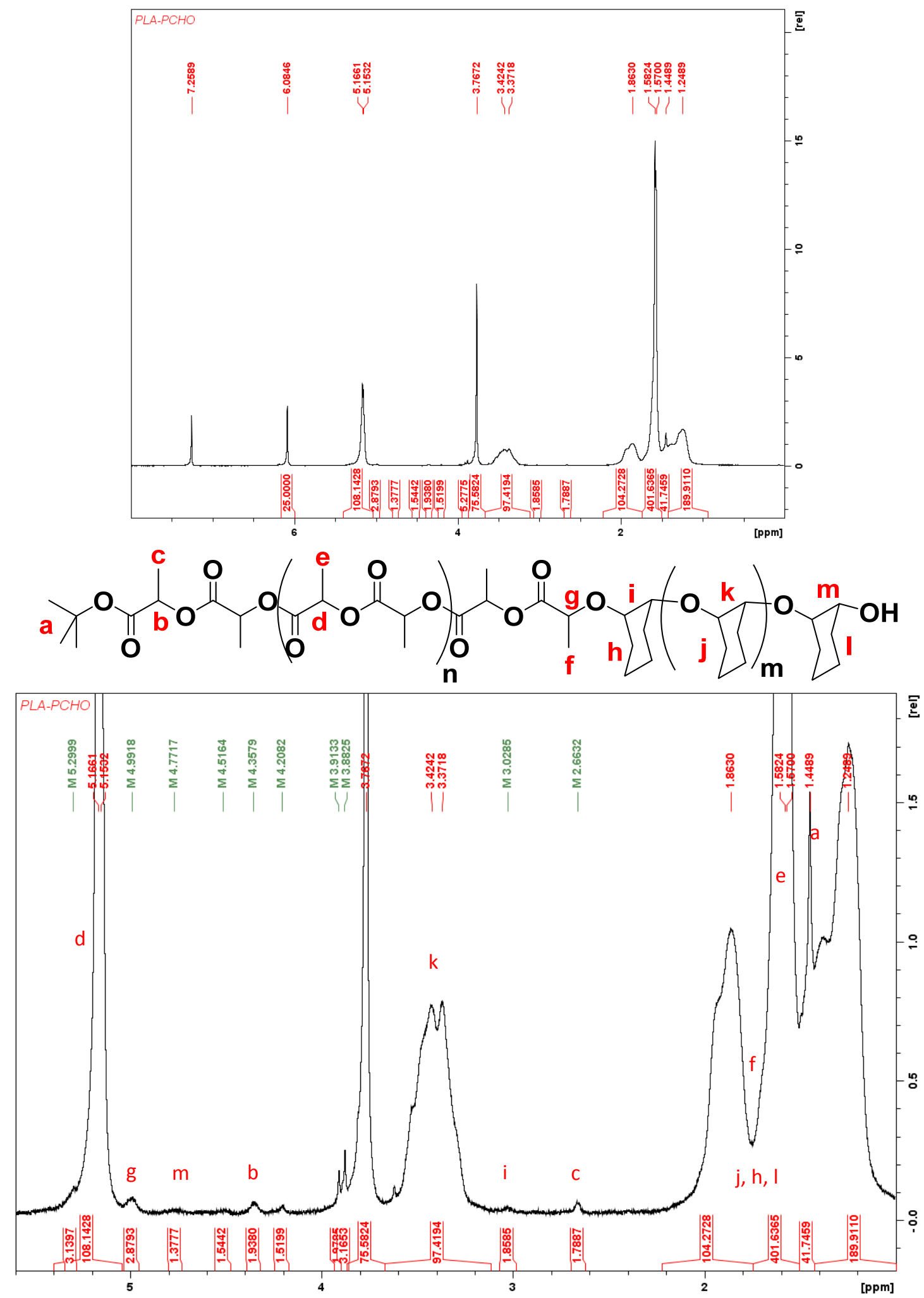

Figure S63. Top: ${ }^{1} \mathrm{H}$ NMR $\left(300 \mathrm{MHz}, 25{ }^{\circ} \mathrm{C}, \mathrm{C}_{6} \mathrm{D}_{6}\right)$ spectrum of the purified PLA-PCHO polymer obtained by polymerization of 100 equivalents of L-lactide and cyclohexene oxide, monomers added sequentially, using catalyst redox switch "red-ox". Bottom: Magnified peaks to show the assignment of the protons corresponding to the junction between blocks. 


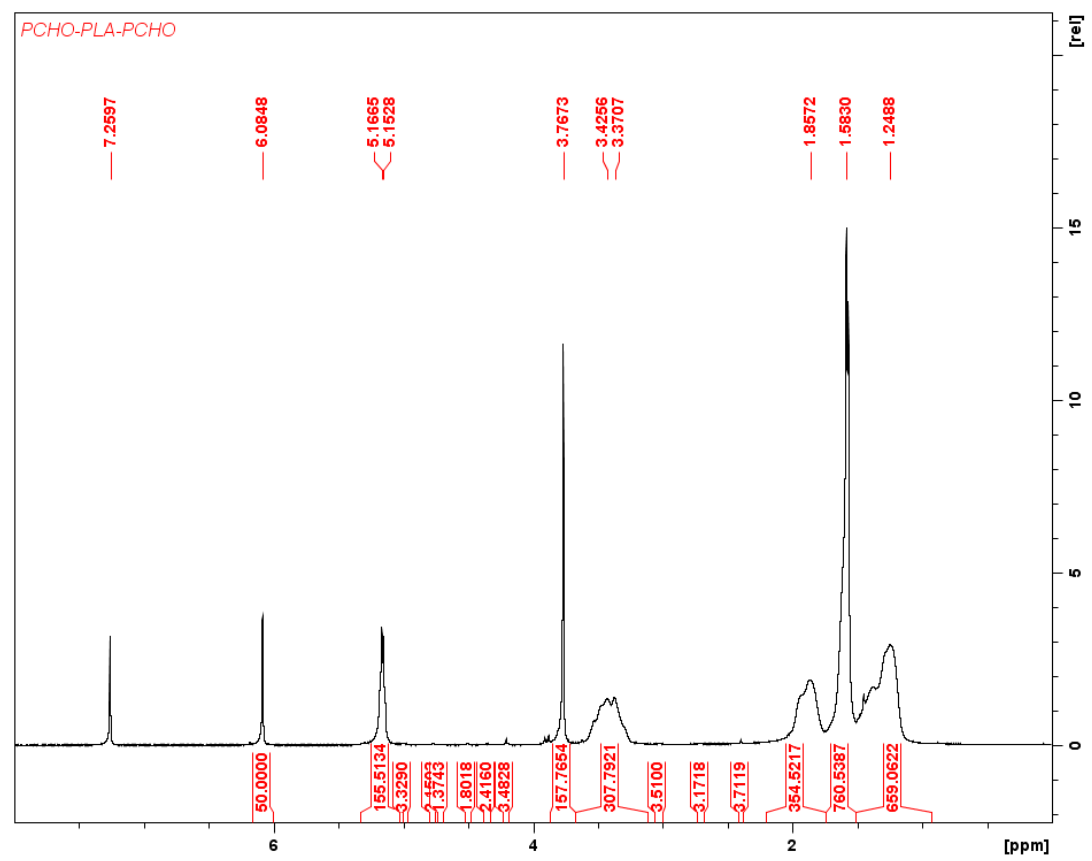

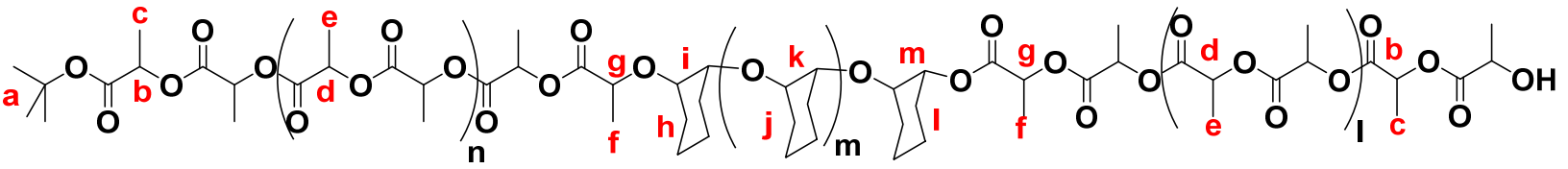

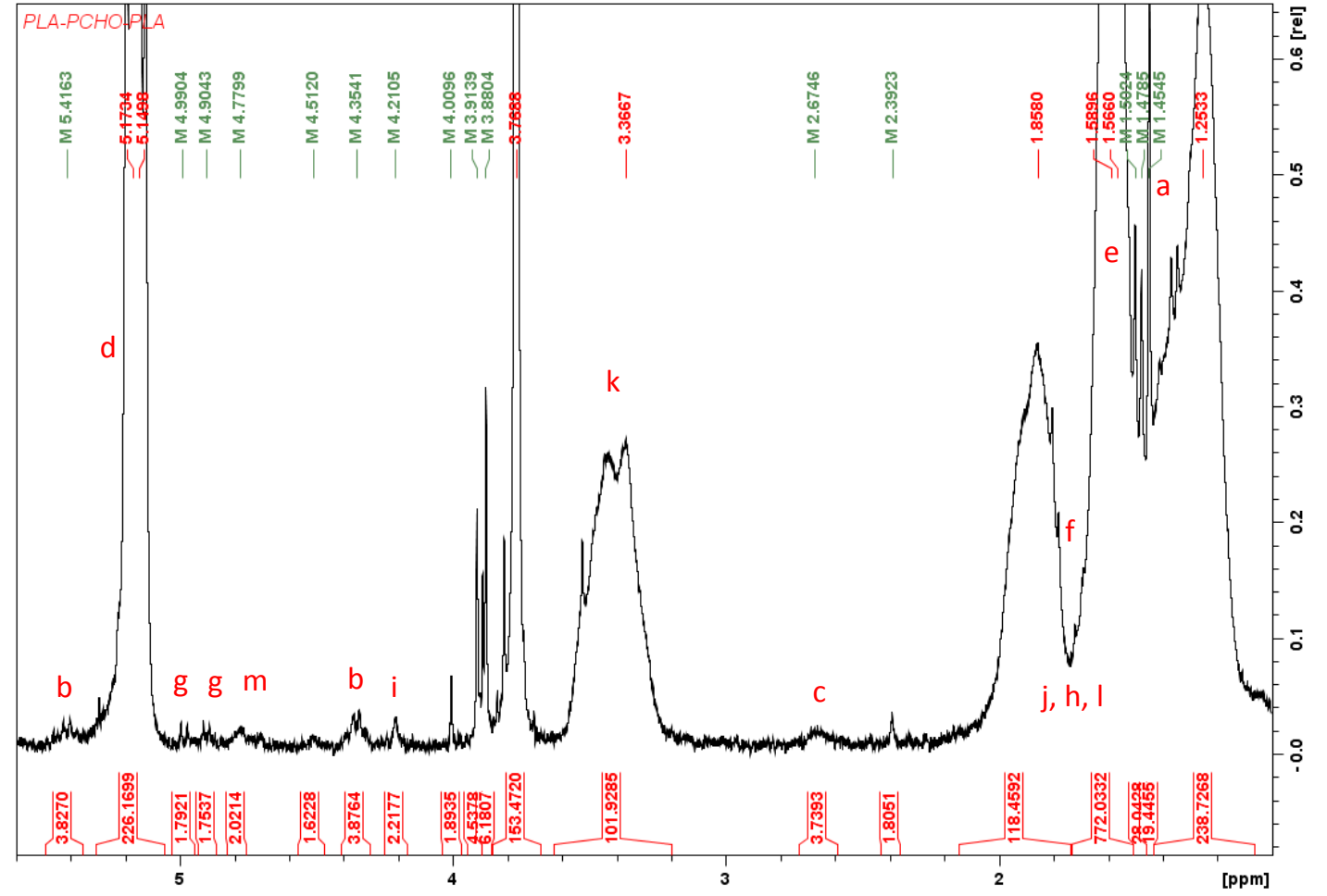

Figure S64. Top: ${ }^{1} \mathrm{H}$ NMR $\left(300 \mathrm{MHz}, 25{ }^{\circ} \mathrm{C}, \mathrm{C}_{6} \mathrm{D}_{6}\right)$ spectrum of the purified PLA-PCHO-PLA polymer obtained by polymerization of 100 equivalents of L-lactide and cyclohexene oxide, monomers added sequentially, using catalyst redox switch "red-ox-red". Bottom: Magnified peaks to show the assignment of the protons corresponding to the junction between blocks. 

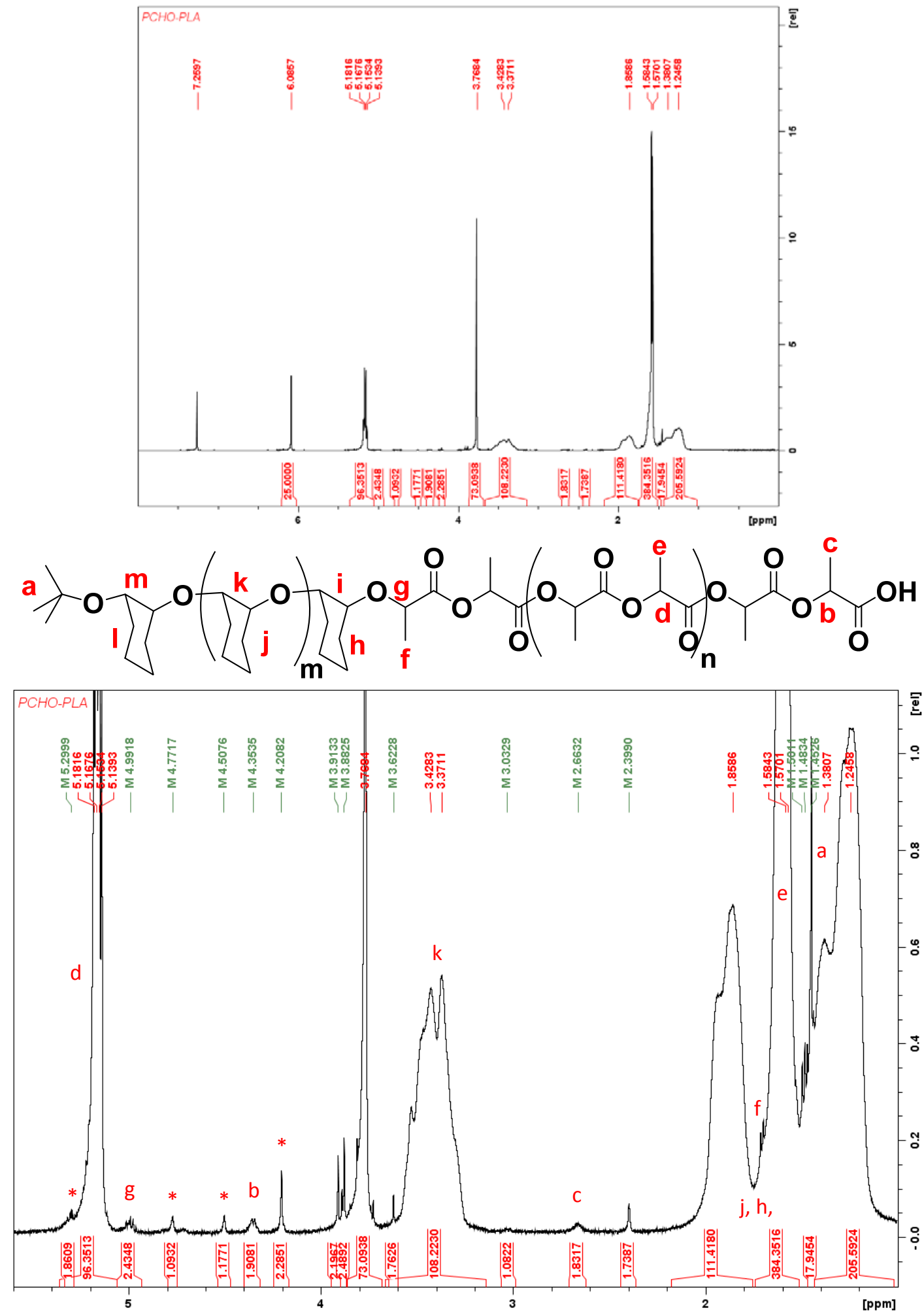

Figure S65. Top: ${ }^{1} \mathrm{H}$ NMR $\left(300 \mathrm{MHz}, 25{ }^{\circ} \mathrm{C}, \mathrm{C}_{6} \mathrm{D}_{6}\right)$ spectrum of the purified PCHO-PLA polymer obtained by polymerization of 100 equivalents of cyclohexene oxide and L-lactide monomers added sequentially, using catalyst redox switch "ox-red". Bottom: Magnified peaks to show the assignment of the protons corresponding to the junction between blocks. *unable to assign. Some of the peaks showed correlations to LA methine carbons. HMBC inconclusive. 

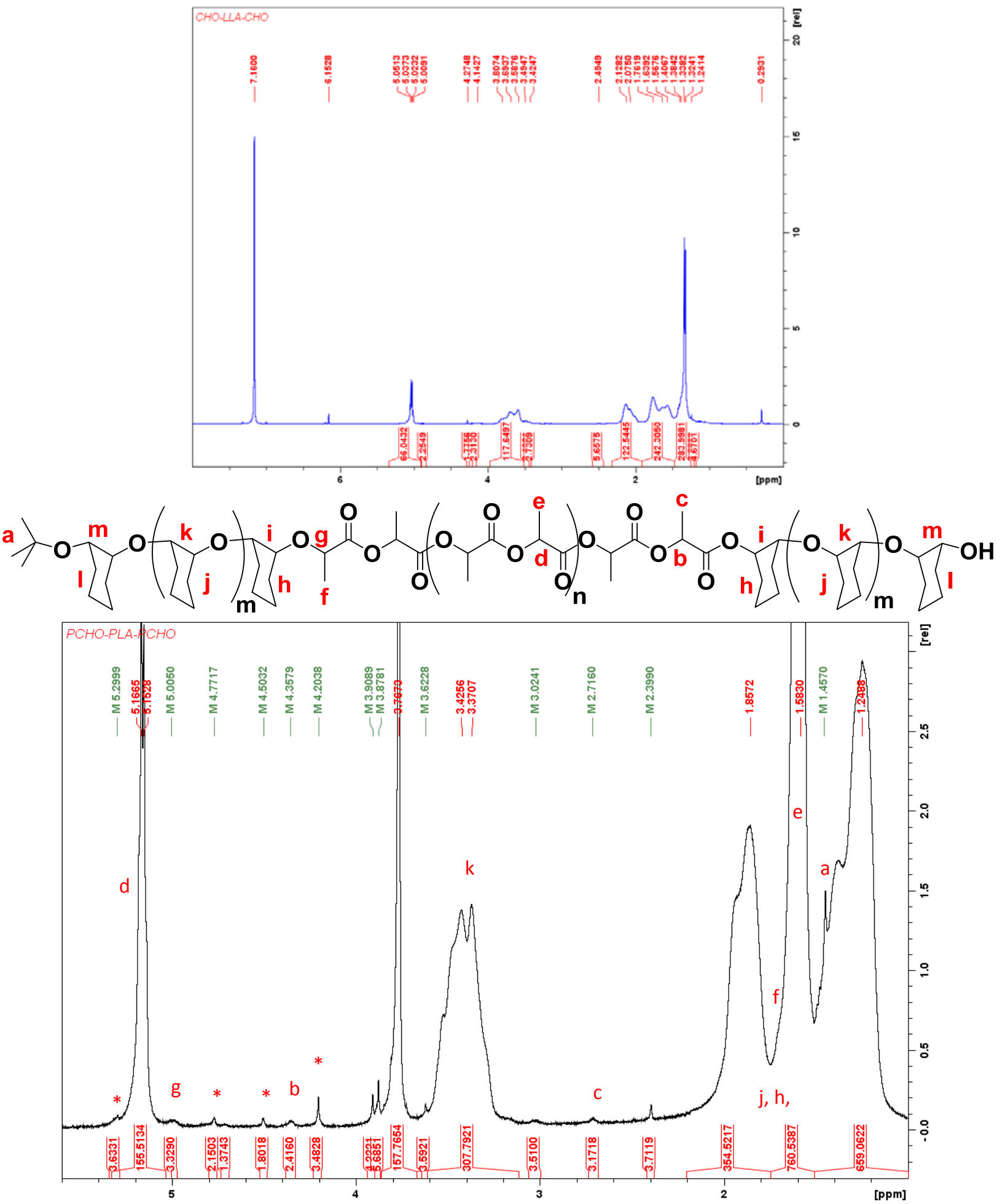

Figure S66. Top: ${ }^{1} \mathrm{H}$ NMR $\left(300 \mathrm{MHz}, 25{ }^{\circ} \mathrm{C}, \mathrm{C}_{6} \mathrm{D}_{6}\right)$ spectrum of the purified PCHO-PLA-PCHO polymer obtained by polymerization of 100 equivalents of cyclohexene oxide and L-lactide monomers added sequentially, using catalyst redox switch "ox-red-ox". Bottom: Magnified peaks to show the assignment of the protons corresponding to the junction between blocks. *unable to assign. Some of the peaks showed correlations to LA methine carbons. HMBC inconclusive. 


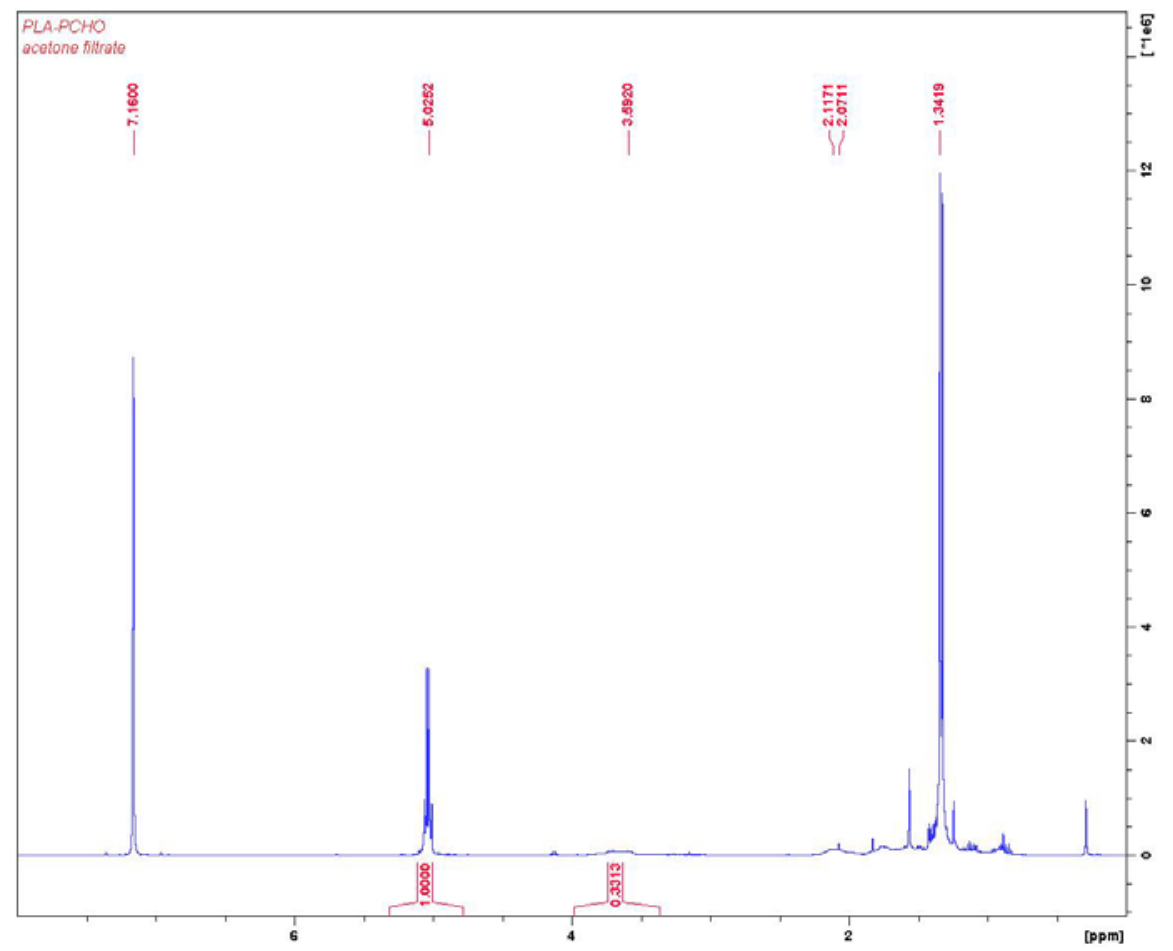

Figure S67. Table S2, entry 2. Acetone filtrate from selective precipitation of PLA-PCHO. ${ }^{1} \mathrm{H}$ NMR $\left(300 \mathrm{MHz}, 25{ }^{\circ} \mathrm{C}, \mathrm{C}_{6} \mathrm{D}_{6}\right.$ ), $\delta$ (ppm): 5.03 (q, 2H, $\left.\mathrm{CHCH}_{3} \mathrm{PLA}\right), 3.59$ (m, 2H, COCH PCHO), 2.11-2.07 (m, 2H, $\left.\mathrm{COCHCH}_{2} \mathrm{PCHO}\right), 1.34$ (d, 6H, $\left.\mathrm{CHCH}_{3} \mathrm{PLA}\right)$.

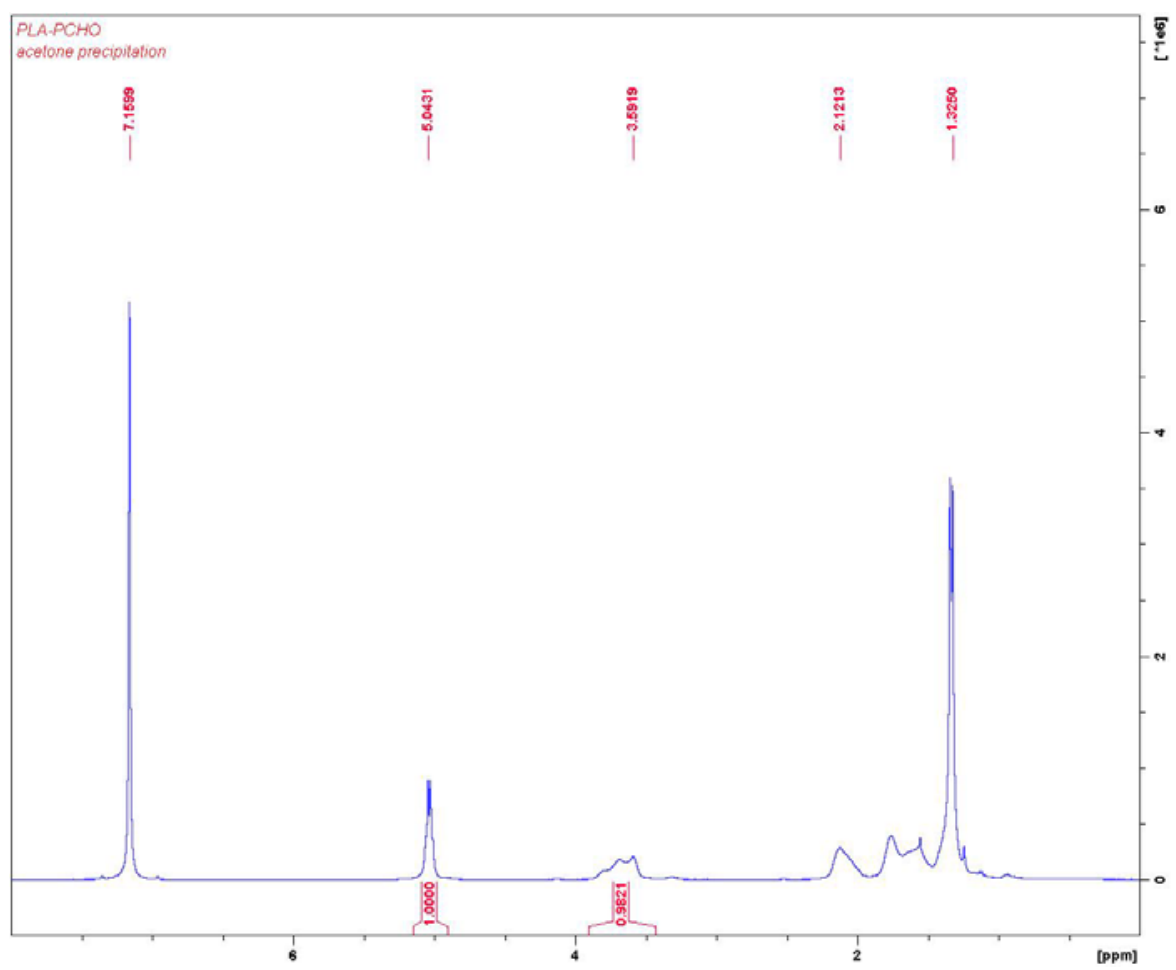

Figure S68. Table S2, entry 3. Acetone precipitate from selective precipitation of PLA-PCHO. ${ }^{1} \mathrm{H}$ NMR $\left(300 \mathrm{MHz}, 2{ }^{\circ} \mathrm{C}, \mathrm{C}_{6} \mathrm{D}_{6}\right), \delta(\mathrm{ppm}): 5.04$ (q, 2H, $\left.\mathrm{CHCH}_{3} \mathrm{PLA}\right), 3.59$ (m, 2H, COCH $\mathrm{PCHO}), 2.12\left(\mathrm{~m}, 2 \mathrm{H}, \mathrm{COCHCH}_{2} \mathrm{PCHO}\right), 1.33$ ( $\left.\mathrm{m}, 2 \mathrm{H}, \mathrm{COCHCH}_{2} \mathrm{CH}_{2} \mathrm{PCHO}\right)$. 


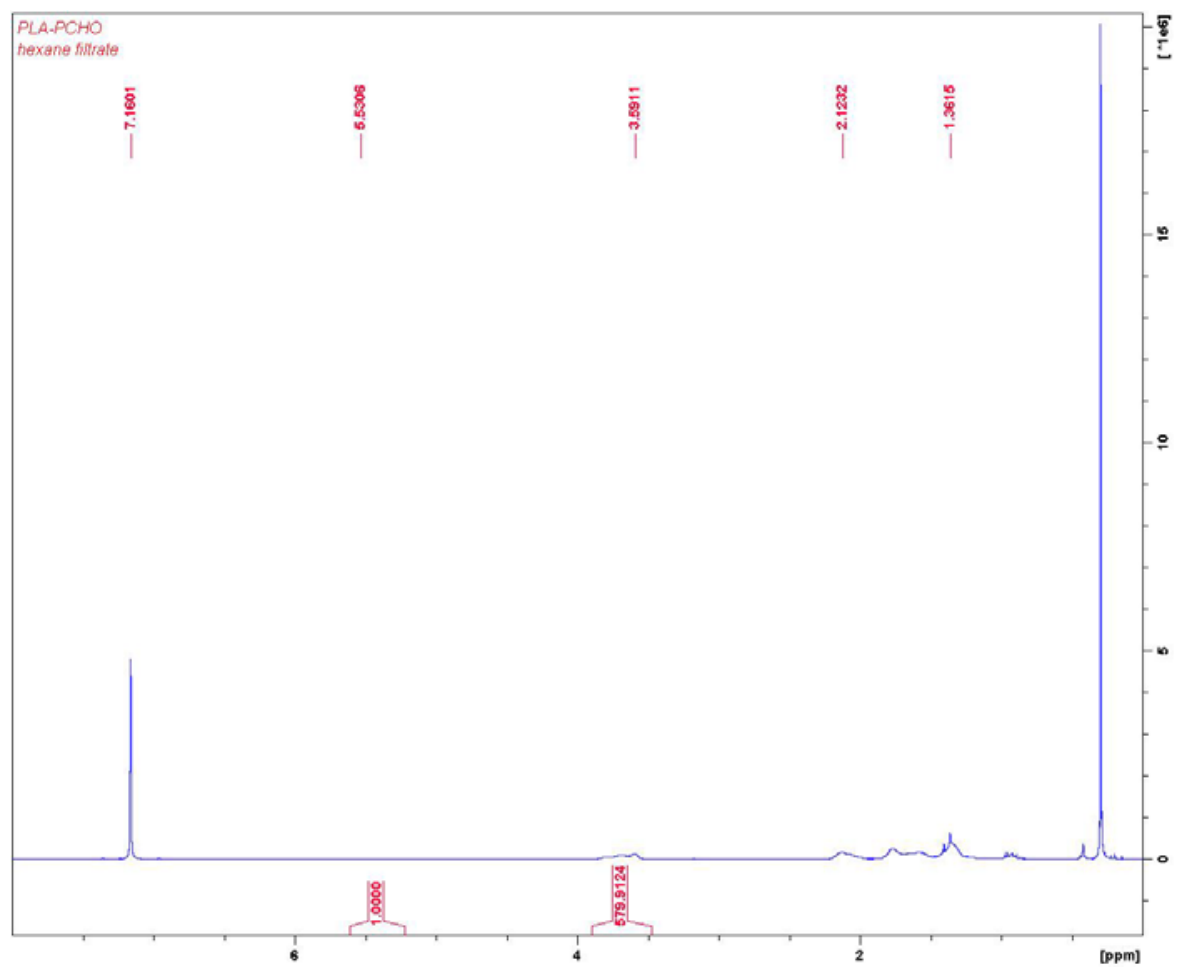

Figure S69. Table S2, entry 4. Hexanes filtrate from selective precipitation of PLA-PCHO. ${ }^{1} \mathrm{H}$ NMR (300 MHz, $25{ }^{\circ} \mathrm{C}, \mathrm{C}_{6} \mathrm{D}_{6}$ ), $\delta(\mathrm{ppm}): 5.53$ (q, 2H, $\left.\mathrm{CHCH}_{3} \mathrm{PLA}\right), 3.59$ (m, 2H, COCH $\mathrm{PCHO}), 2.12\left(\mathrm{~m}, 2 \mathrm{H}, \mathrm{COCHCH}_{2} \mathrm{PCHO}\right), 1.36$ (d, 6H, $\left.\mathrm{CHCH}_{3} \mathrm{PLA}\right)$.

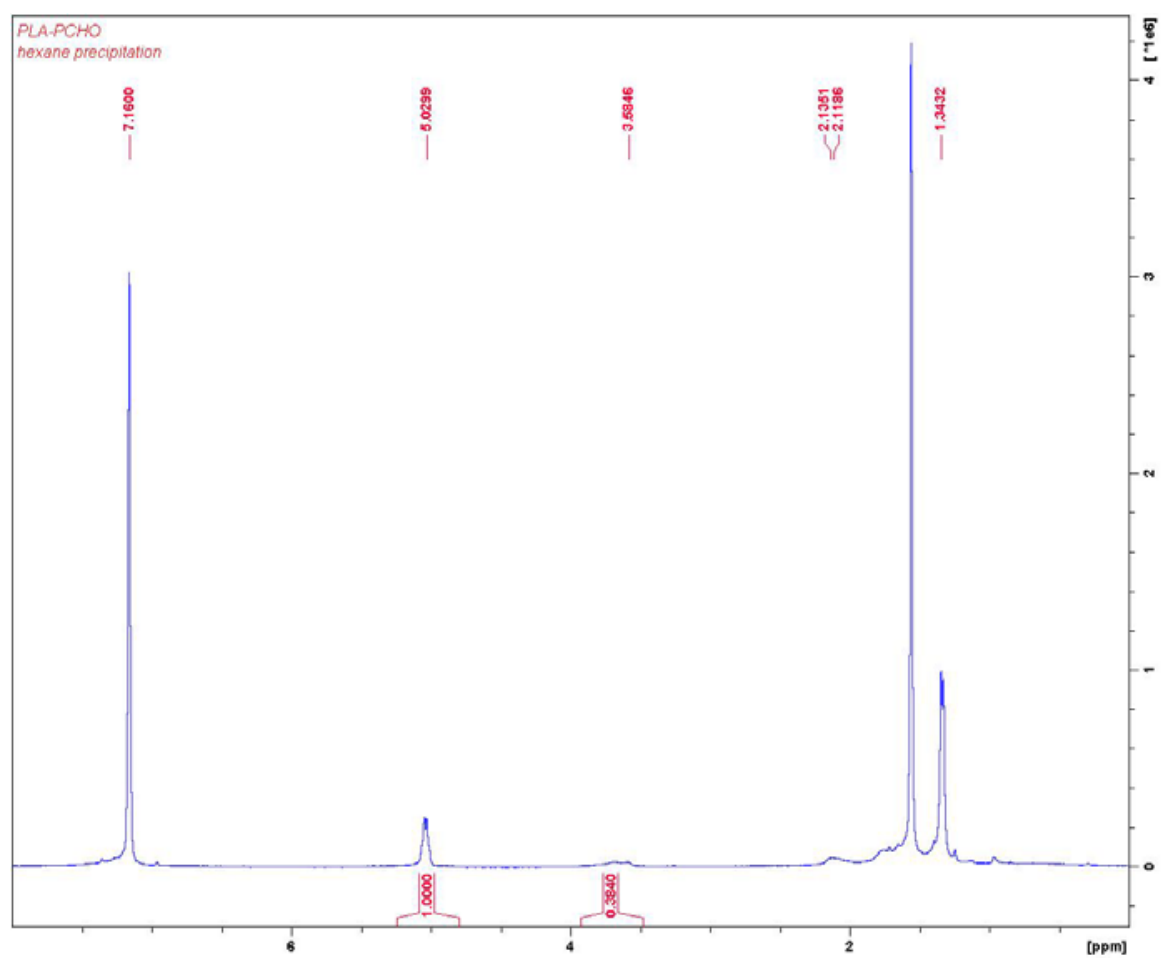

Figure S70. Table S2, entry 5. Hexanes precipitate from selective precipitation of PLA-PCHO. ${ }^{1} \mathrm{H}$ NMR $\left(300 \mathrm{MHz}, 25^{\circ} \mathrm{C}, \mathrm{C}_{6} \mathrm{D}_{6}\right), \delta(\mathrm{ppm}): 5.03$ (q, 2H, $\left.\mathrm{CHCH}_{3} \mathrm{PLA}\right), 3.58$ (m, 2H, COCH PCHO), 2.12 (m, 2H, $\left.\mathrm{COCHCH}_{2} \mathrm{PCHO}\right), 1.34$ (d, 6H, $\left.\mathrm{CHCH}_{3} \mathrm{PLA}\right)$. 


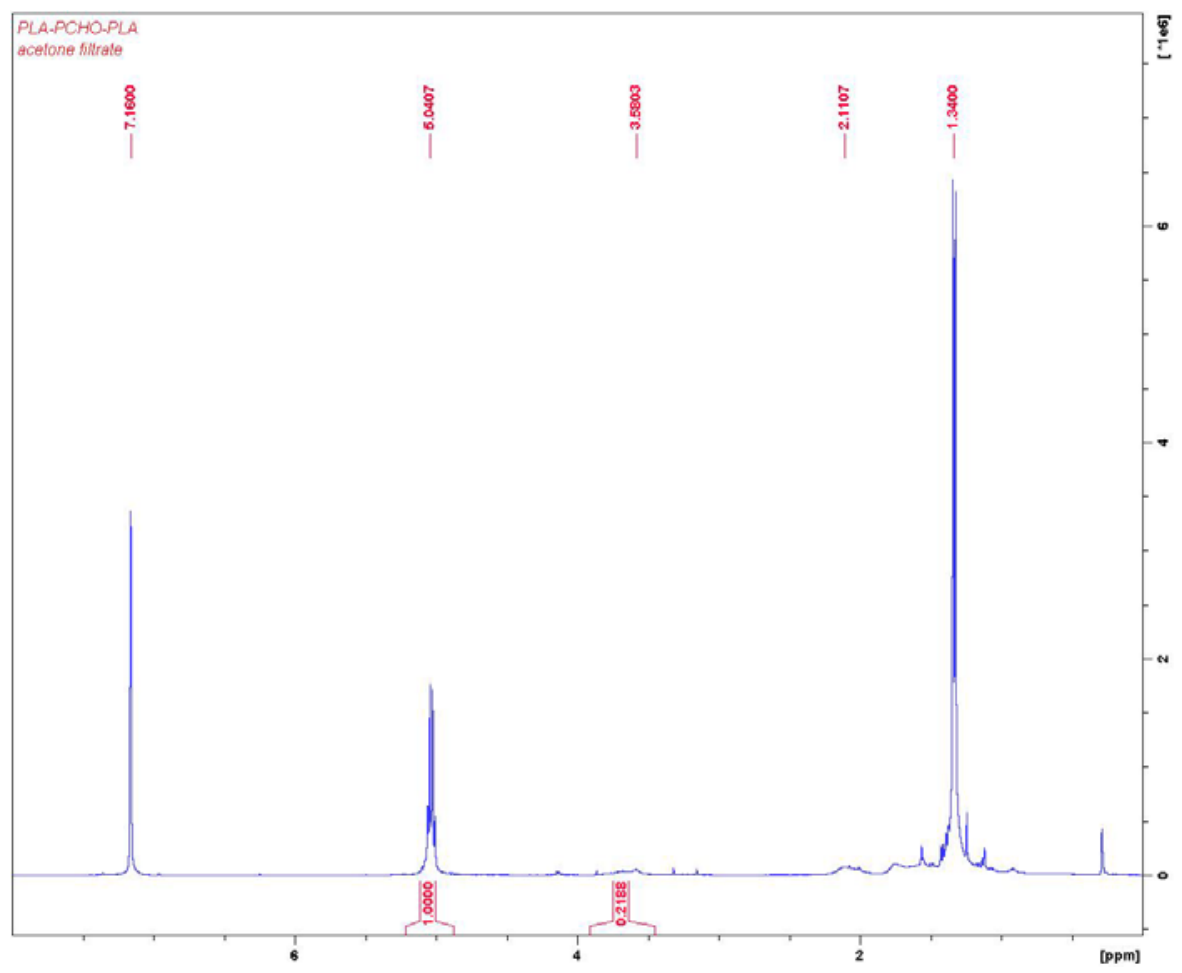

Figure S71. Table S3, entry 2. Acetone filtrate from selective precipitation of PLA-PCHO-PLA. ${ }^{1} \mathrm{H}$ NMR (300 MHz, $\left.25^{\circ}, \mathrm{C}_{6} \mathrm{D}_{6}\right), \delta(\mathrm{ppm}): 5.04$ (q, 2H, $\left.\mathrm{CHCH}_{3} \mathrm{PLA}\right), 3.58(\mathrm{~m}, 2 \mathrm{H}, \mathrm{COCH}$ PCHO), 2.11 (m, 2H, $\left.\mathrm{COCHCH}_{2} \mathrm{PCHO}\right), 1.34$ (d, 6H, $\left.\mathrm{CHCH}_{3} \mathrm{PLA}\right)$.

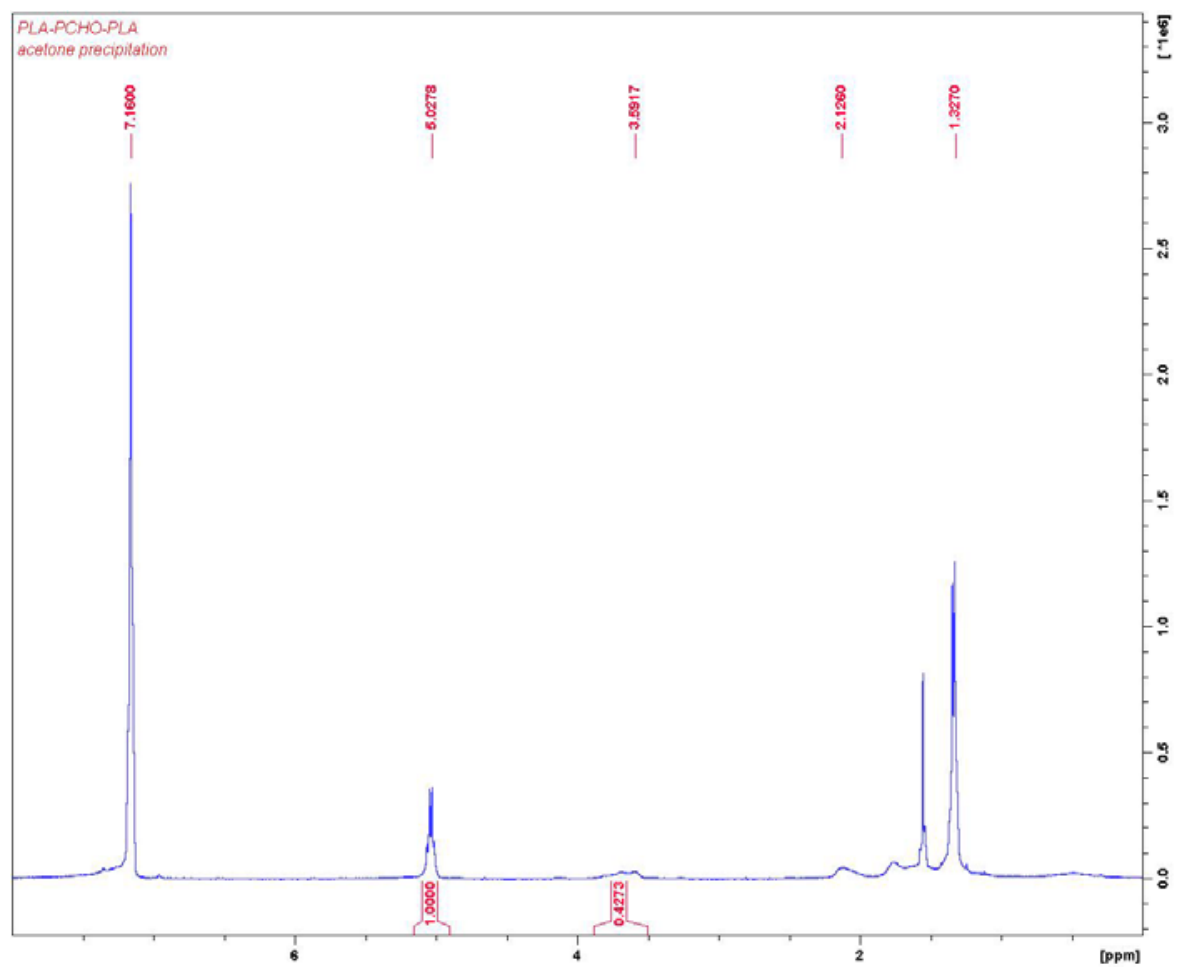

Figure S72. Table S3, entry 3. Acetone precipitate from selective precipitation of PLA-PCHOPLA. ${ }^{1} \mathrm{H}$ NMR (300 MHz, $25{ }^{\circ} \mathrm{C}, \mathrm{C}_{6} \mathrm{D}_{6}$ ), $\delta(\mathrm{ppm}): 5.03$ (q, 2H, CHCH3 PLA), 3.58 (m, 2H, $\mathrm{COCH} \mathrm{PCHO}$ ), 2.12 (m, 2H, $\left.\mathrm{COCHCH}_{2} \mathrm{PCHO}\right), 1.33$ (d, 6H, $\left.\mathrm{CHCH}_{3} \mathrm{PLA}\right)$. 


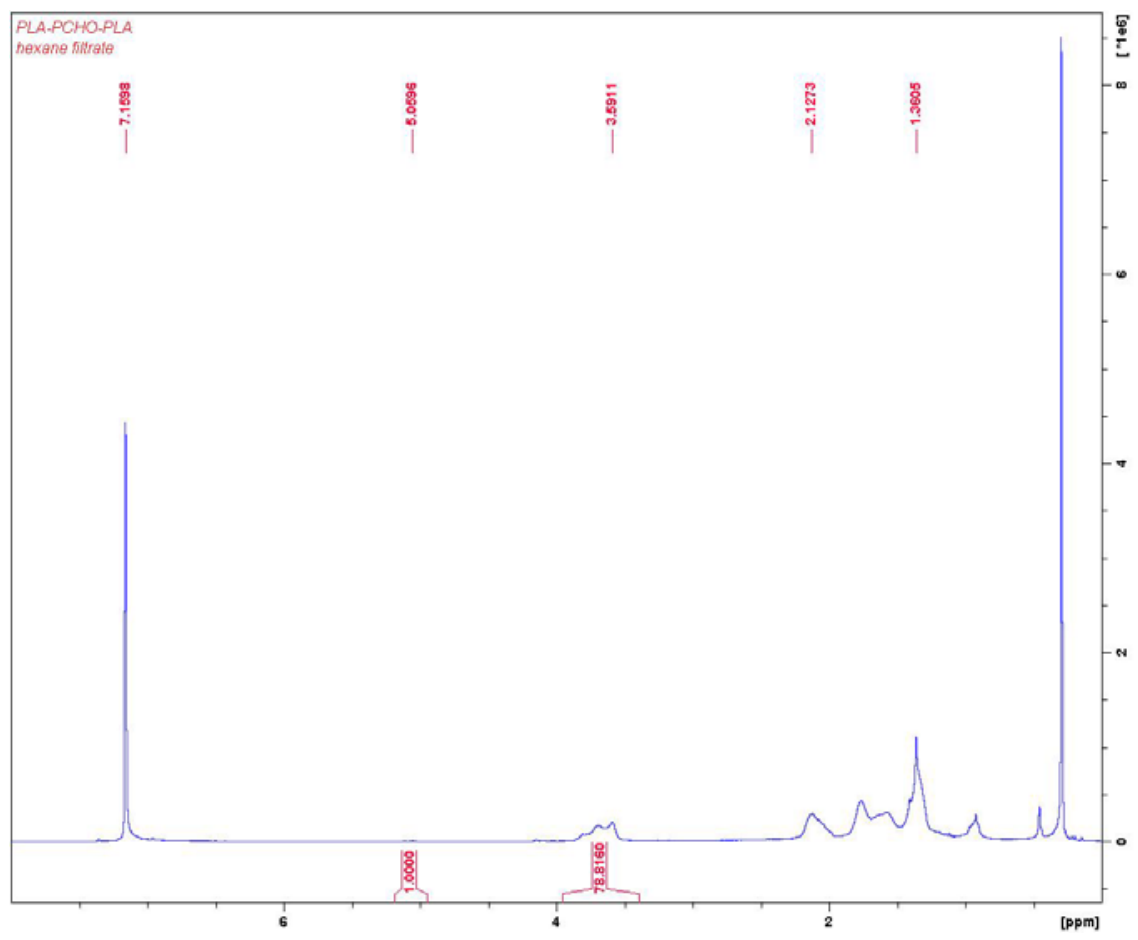

Figure S73. Table S3, entry 4. Hexanes filtrate from selective precipitation of PLA-PCHO-PLA. ${ }^{1} \mathrm{H}$ NMR (300 MHz, $25^{\circ} \mathrm{C}, \mathrm{C}_{6} \mathrm{D}_{6}$ ), $\delta$ (ppm): 5.06 (q, 2H, $\left.\mathrm{CHCH}_{3} \mathrm{PLA}\right), 3.58$ (m, 2H, COCH PCHO), 2.13 (m, 2H, COCHCH 2 PCHO), 1.36 (d, 6H, $\mathrm{CHCH}_{3} \mathrm{PLA}$ ).

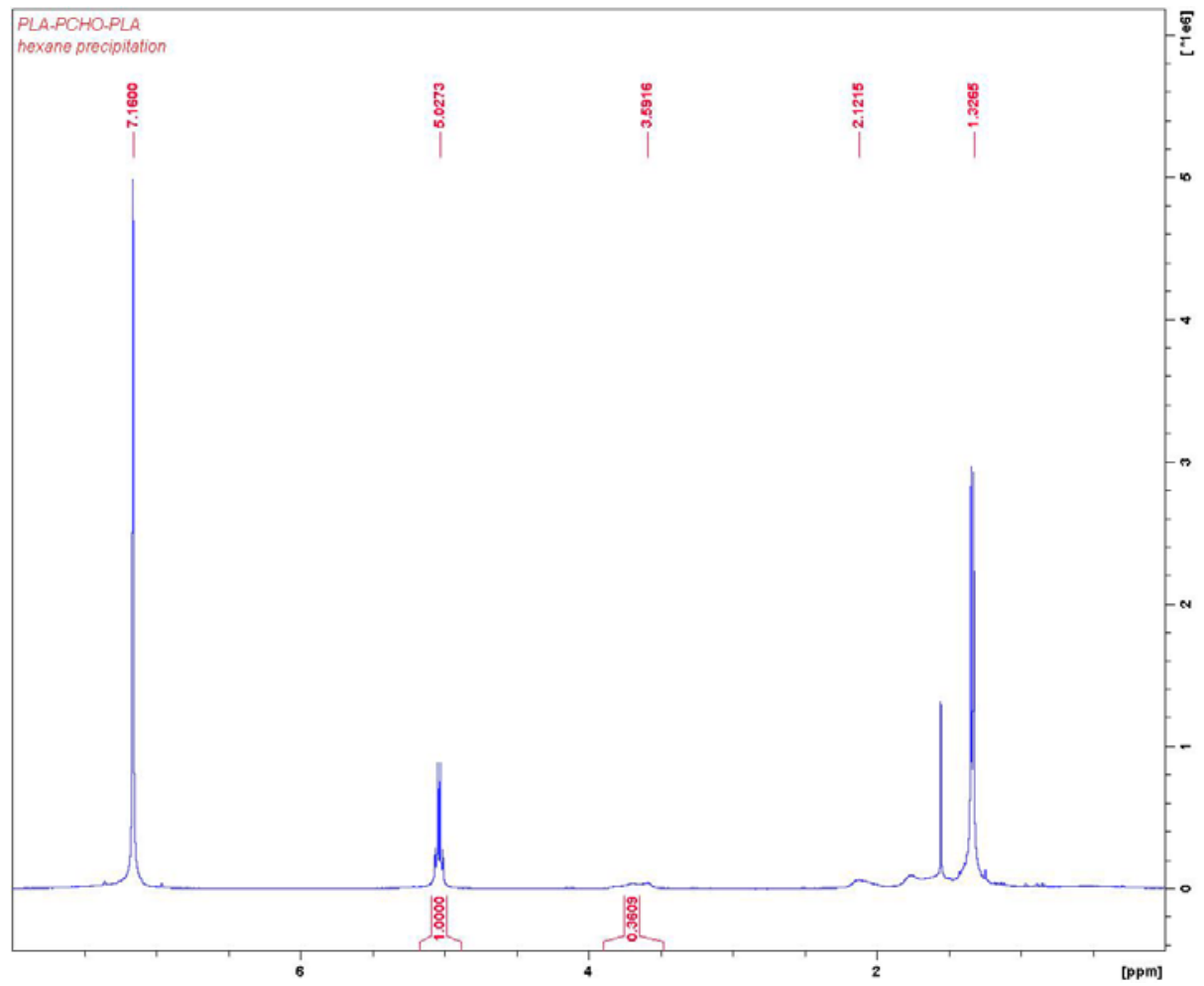

Figure S74. Table S3, entry 5. Hexanes precipitate from selective precipitation of PLA-PCHOPLA. ${ }^{1} \mathrm{H}$ NMR $\left(300 \mathrm{MHz}, 25{ }^{\circ} \mathrm{C}, \mathrm{C}_{6} \mathrm{D}_{6}\right), \delta$ (ppm): 5.03 (q, 2H, $\left.\mathrm{CHCH}_{3} \mathrm{PLA}\right), 3.59$ (m, 2H, $\mathrm{COCH}$ PCHO), 2.12 (m, 2H, $\left.\mathrm{COCHCH}_{2} \mathrm{PCHO}\right), 1.33$ (d, 6H, $\left.\mathrm{CHCH}_{3} \mathrm{PLA}\right)$. 


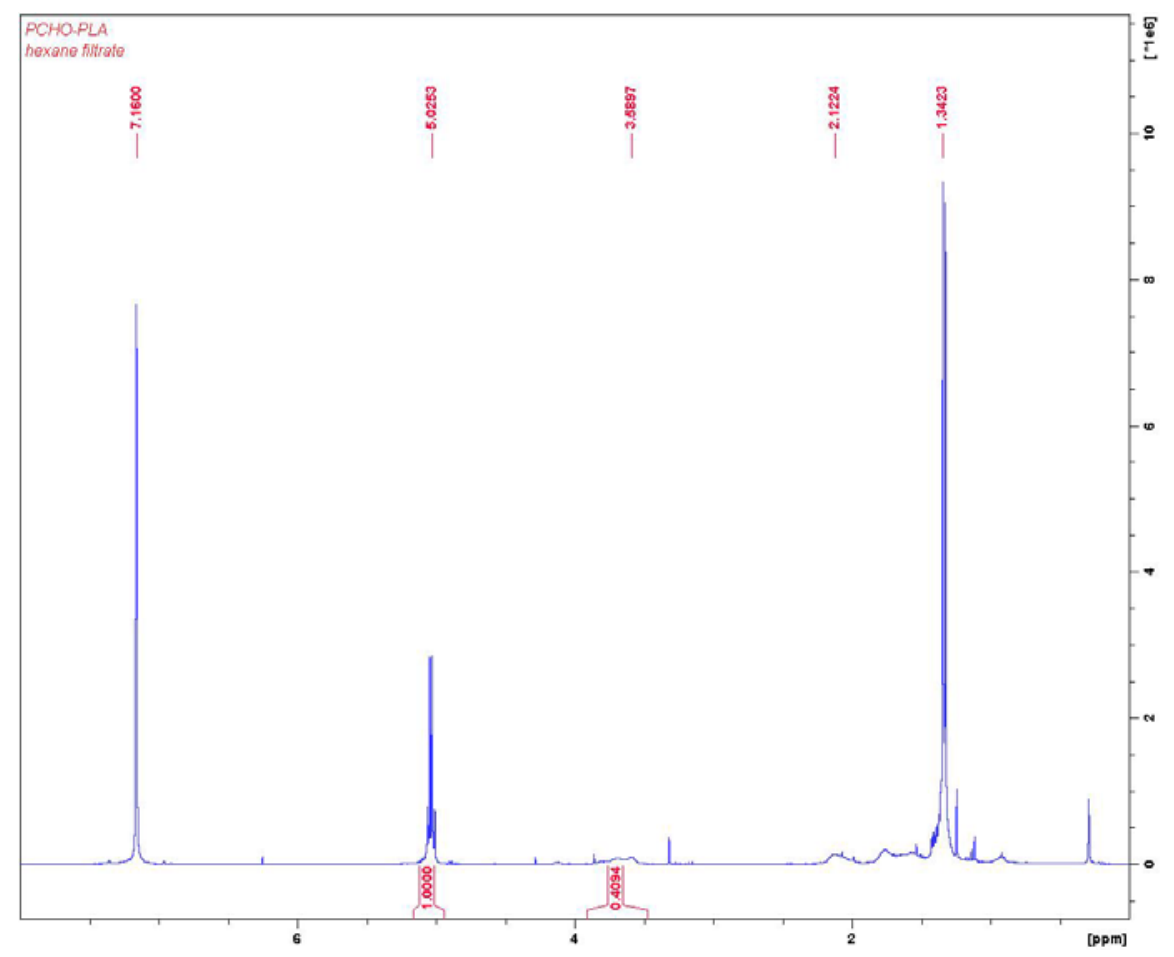

Figure S75. Table S4, entry 2. Acetone filtrate from selective precipitation of PCHO-PLA. ${ }^{1} \mathrm{H}$ NMR $\left(300 \mathrm{MHz}, 25{ }^{\circ} \mathrm{C}, \mathrm{C}_{6} \mathrm{D}_{6}\right), \delta(\mathrm{ppm}): 5.03$ (q, 2H, $\left.\mathrm{CHCH}_{3} \mathrm{PLA}\right), 3.58(\mathrm{~m}, 2 \mathrm{H}, \mathrm{COCH}$ $\mathrm{PCHO}), 2.12$ (m, 2H, $\left.\mathrm{COCHCH}_{2} \mathrm{PCHO}\right), 1.34$ (d, 6H, $\left.\mathrm{CHCH}_{3} \mathrm{PLA}\right)$.

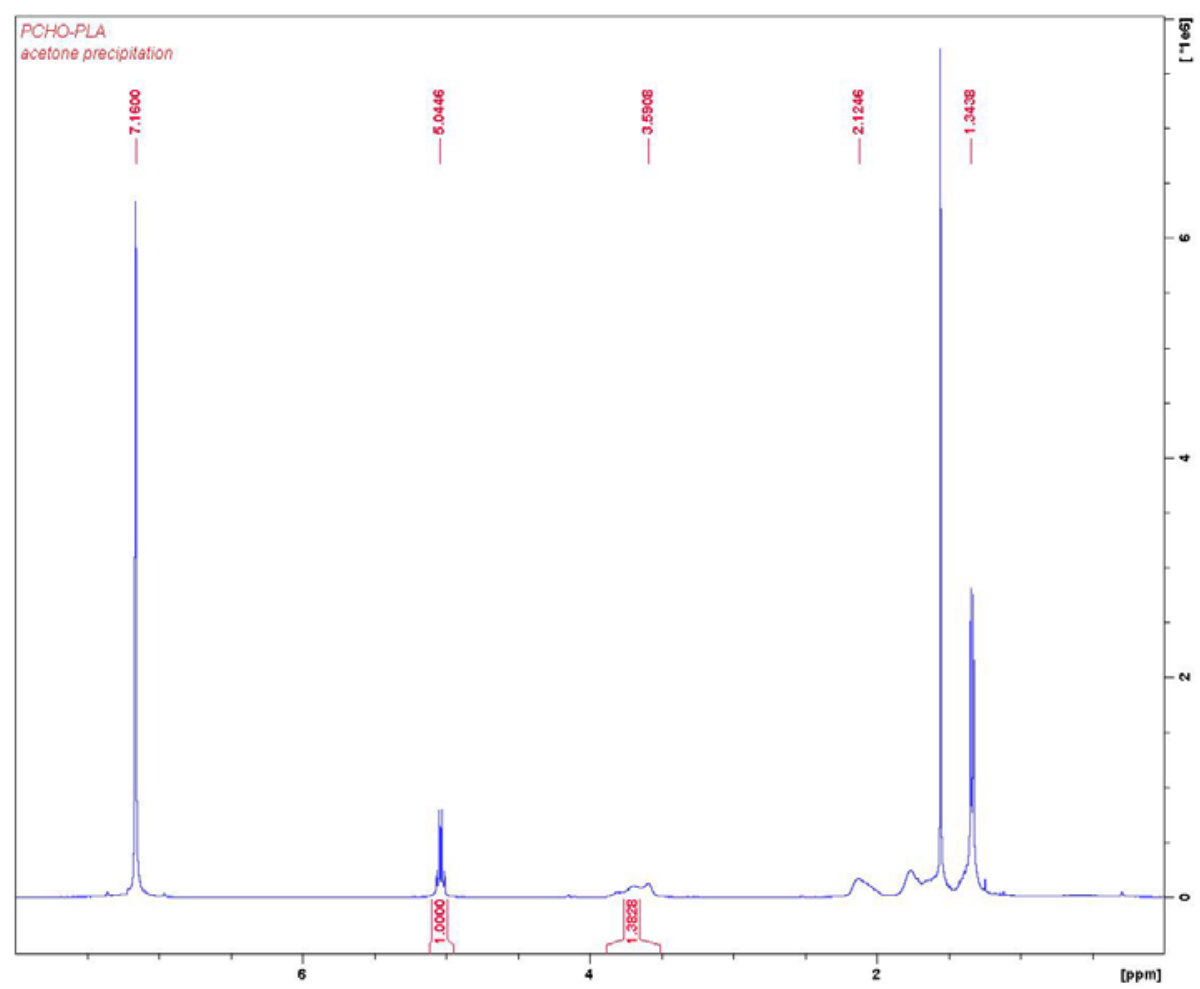

Figure S76. Table S4, entry 3 . Acetone precipitate from selective precipitation of PCHO-PLA. ${ }^{1} \mathrm{H}$ NMR $\left(300 \mathrm{MHz}, 2{ }^{\circ} \mathrm{C}, \mathrm{C}_{6} \mathrm{D}_{6}\right), \delta(\mathrm{ppm}): 5.04$ (q, 2H, $\left.\mathrm{CHCH}_{3} \mathrm{PLA}\right), 3.59$ (m, 2H, COCH PCHO), 2.12 (m, 2H, $\left.\mathrm{COCHCH}_{2} \mathrm{PCHO}\right), 1.34$ (d, 6H, $\mathrm{CHCH}_{3} \mathrm{PLA}$ ). 


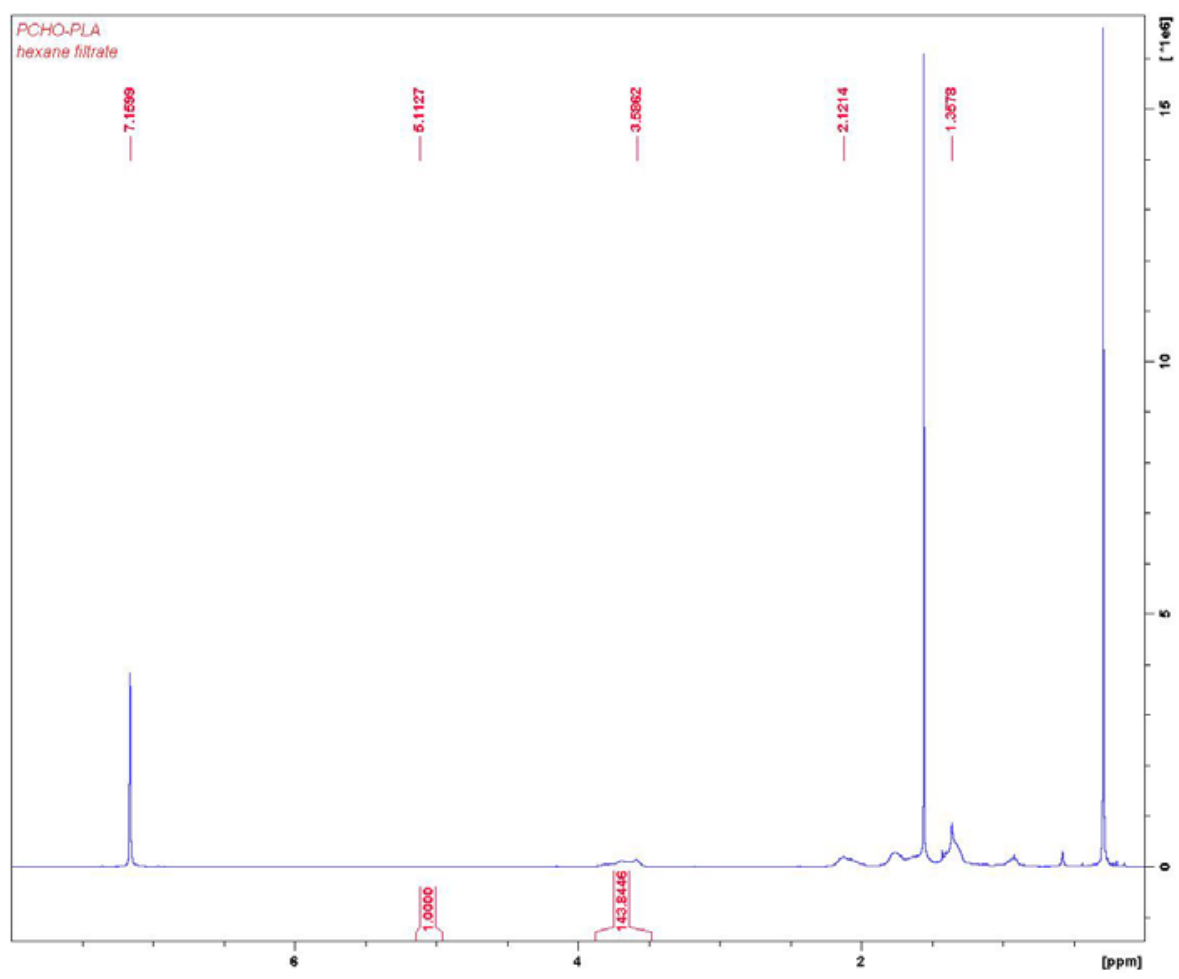

Figure S77. Table S4, entry 4. Hexanes filtrate from selective precipitation of PCHO-PLA. ${ }^{1} \mathrm{H}$ NMR $\left(300 \mathrm{MHz}, 25{ }^{\circ} \mathrm{C}, \mathrm{C}_{6} \mathrm{D}_{6}\right), \delta$ (ppm): 5.11 (q, 2H, $\left.\mathrm{CHCH}_{3} \mathrm{PLA}\right), 3.59$ (m, 2H, COCH $\mathrm{PCHO}), 2.12$ (m, 2H, $\left.\mathrm{COCHCH}_{2} \mathrm{PCHO}\right), 1.36$ (d, 6H, $\left.\mathrm{CHCH}_{3} \mathrm{PLA}\right)$.

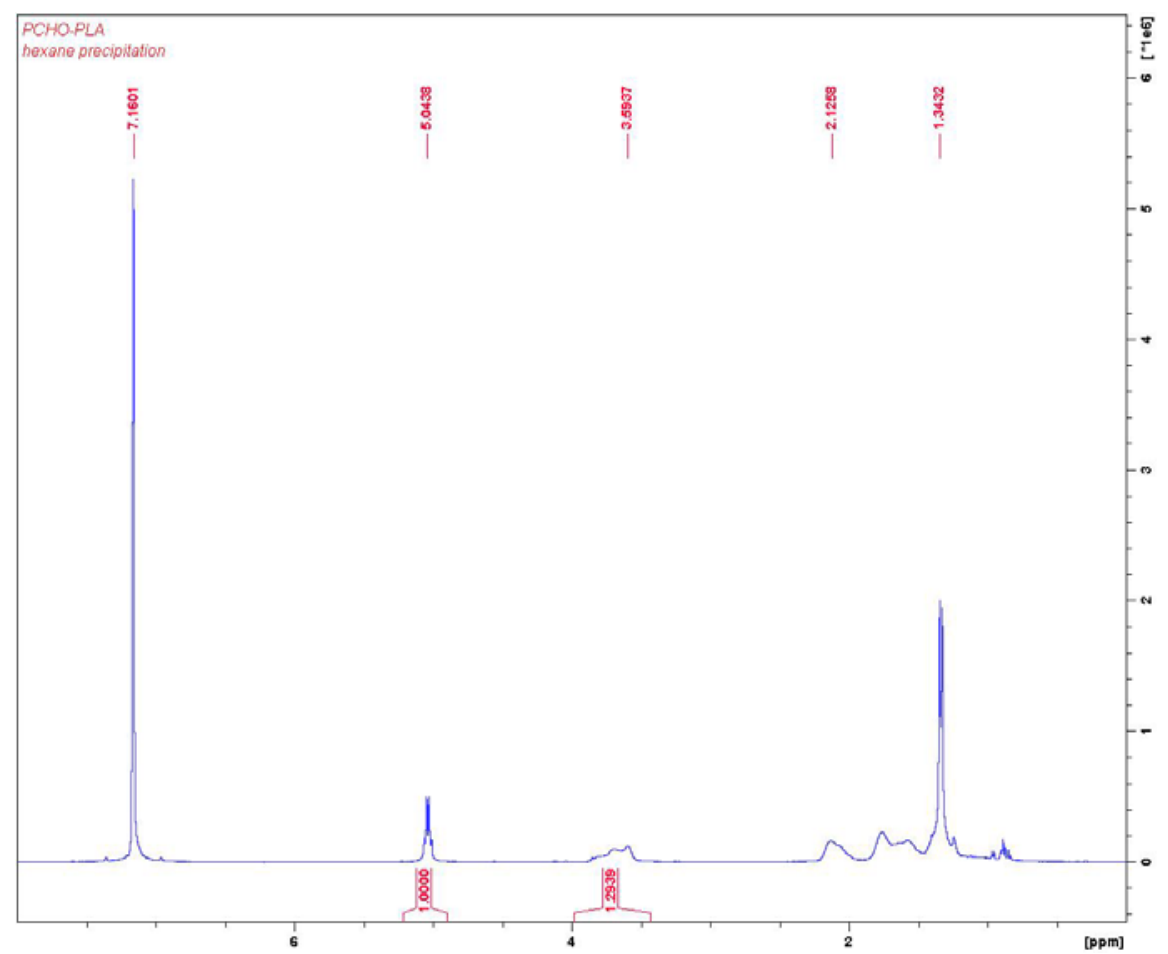

Figure S78. Table S4, entry 5. Hexanes precipitate from selective precipitation of PCHO-PLA. ${ }^{1} \mathrm{H}$ NMR (300 MHz, $25^{\circ} \mathrm{C}, \mathrm{C}_{6} \mathrm{D}_{6}$ ), $\delta(\mathrm{ppm}): 5.03$ (q, 2H, $\left.\mathrm{CHCH}_{3} \mathrm{PLA}\right), 3.58$ (m, 2H, COCH PCHO), 2.13 (m, 2H, $\left.\mathrm{COCHCH}_{2} \mathrm{PCHO}\right), 1.34$ (d, 6H, $\left.\mathrm{CHCH}_{3} \mathrm{PLA}\right)$. 


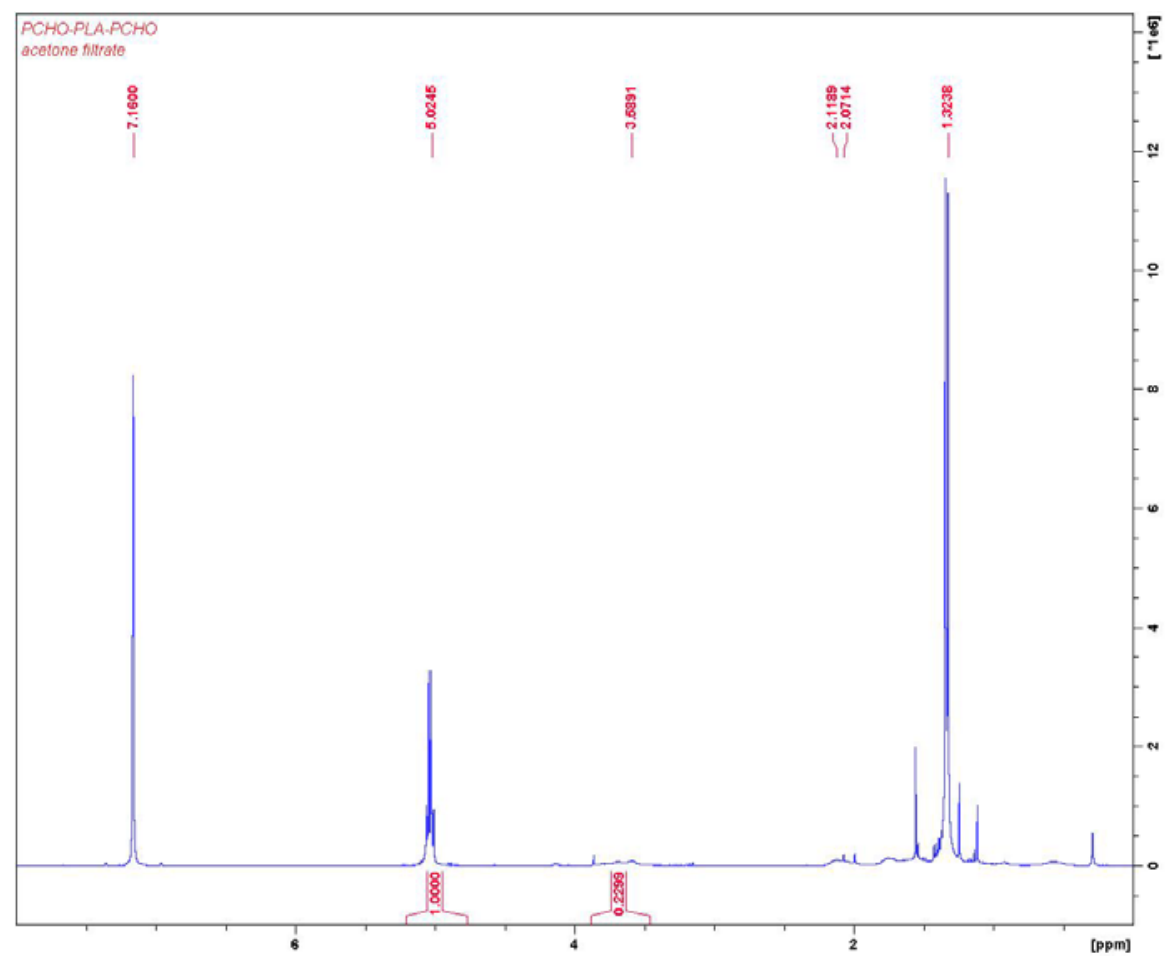

Figure S79. Table S5, entry 2. Acetone filtrate from selective precipitation of PCHO-PLAPCHO. ${ }^{1} \mathrm{H}$ NMR $\left(300 \mathrm{MHz}, 25{ }^{\circ} \mathrm{C}, \mathrm{C}_{6} \mathrm{D}_{6}\right), \delta(\mathrm{ppm}): 5.02$ (q, 2H, $\left.\mathrm{CHCH}_{3} \mathrm{PLA}\right), 3.59$ (m, 2H, $\mathrm{COCH}$ PCHO), 2.11-2.07 (m, 2H, $\left.\mathrm{COCHCH}_{2} \mathrm{PCHO}\right), 1.33$ (d, 6H, $\mathrm{CHCH}_{3} \mathrm{PLA}$ ).

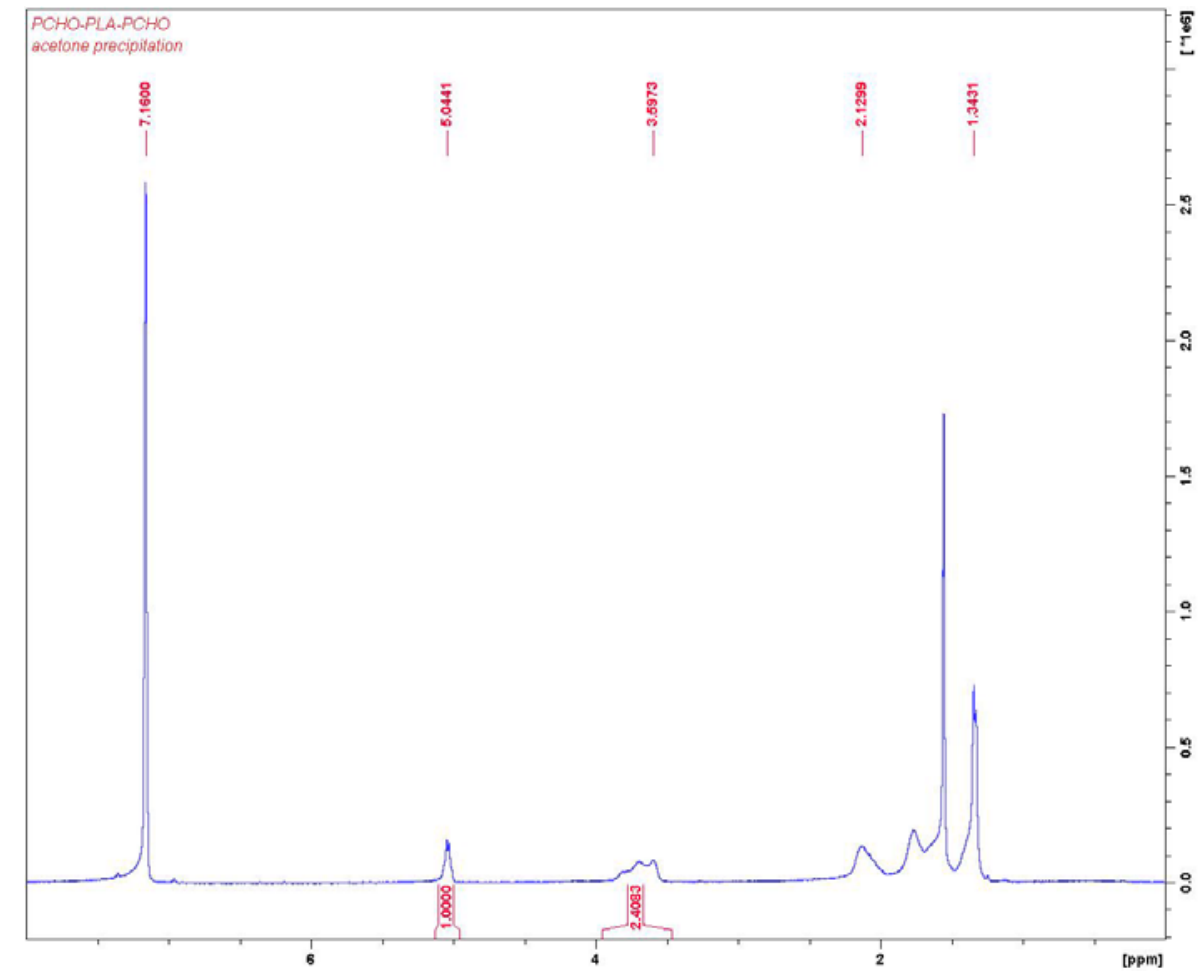

Figure S80. Table S5, entry 3. Acetone precipitate from selective precipitation of PCHO-PLAPCHO. ${ }^{1} \mathrm{H}$ NMR (300 MHz, $\left.25{ }^{\circ} \mathrm{C}, \mathrm{C}_{6} \mathrm{D}_{6}\right), \delta(\mathrm{ppm}): 5.04$ (q, 2H, $\left.\mathrm{CHCH}_{3} \mathrm{PLA}\right), 3.60$ (m, 2H, $\mathrm{COCH} \mathrm{PCHO}$ ), 2.13 (m, 2H, $\left.\mathrm{COCHCH}_{2} \mathrm{PCHO}\right), 1.34$ (d, 6H, $\left.\mathrm{CHCH}_{3} \mathrm{PLA}\right)$. 


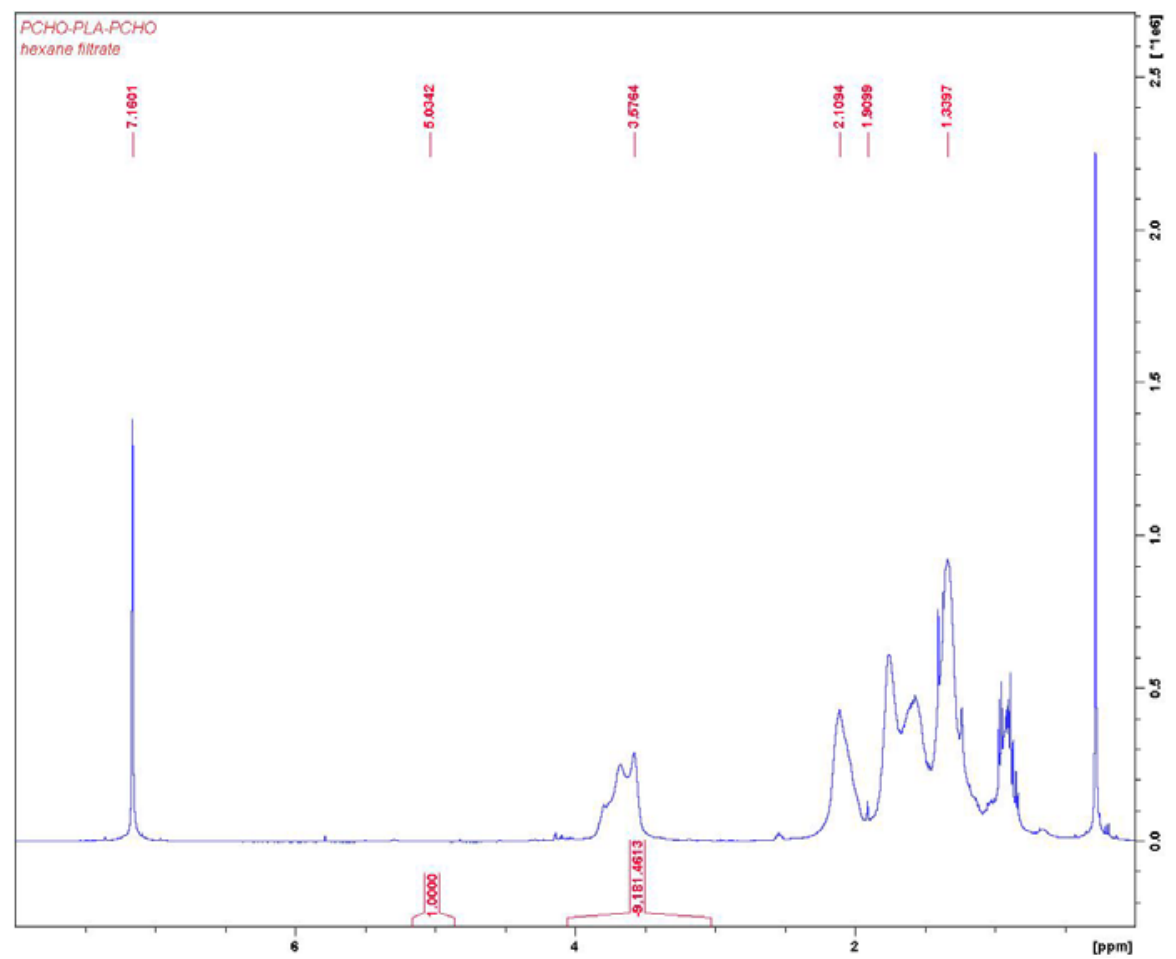

Figure S81. Table S5, entry 4. Hexanes filtrate from selective precipitation of PCHO-PLAPCHO. ${ }^{1} \mathrm{H}$ NMR (300 MHz, $\left.25{ }^{\circ} \mathrm{C}, \mathrm{C}_{6} \mathrm{D}_{6}\right), \delta(\mathrm{ppm}): 5.03$ (q, 2H, $\left.\mathrm{CHCH}_{3} \mathrm{PLA}\right), 3.58$ (m, 2H, $\mathrm{COCH} \mathrm{PCHO}), 2.11\left(\mathrm{~m}, 2 \mathrm{H}, \mathrm{COCHCH}_{2} \mathrm{PCHO}\right), 1.91\left(\mathrm{~m}, 2 \mathrm{H}, \mathrm{COCHCH}_{2} \mathrm{PCHO}\right), 1.34(\mathrm{~d}, 6 \mathrm{H}$, $\left.\mathrm{CHCH}_{3} \mathrm{PLA}\right)$.

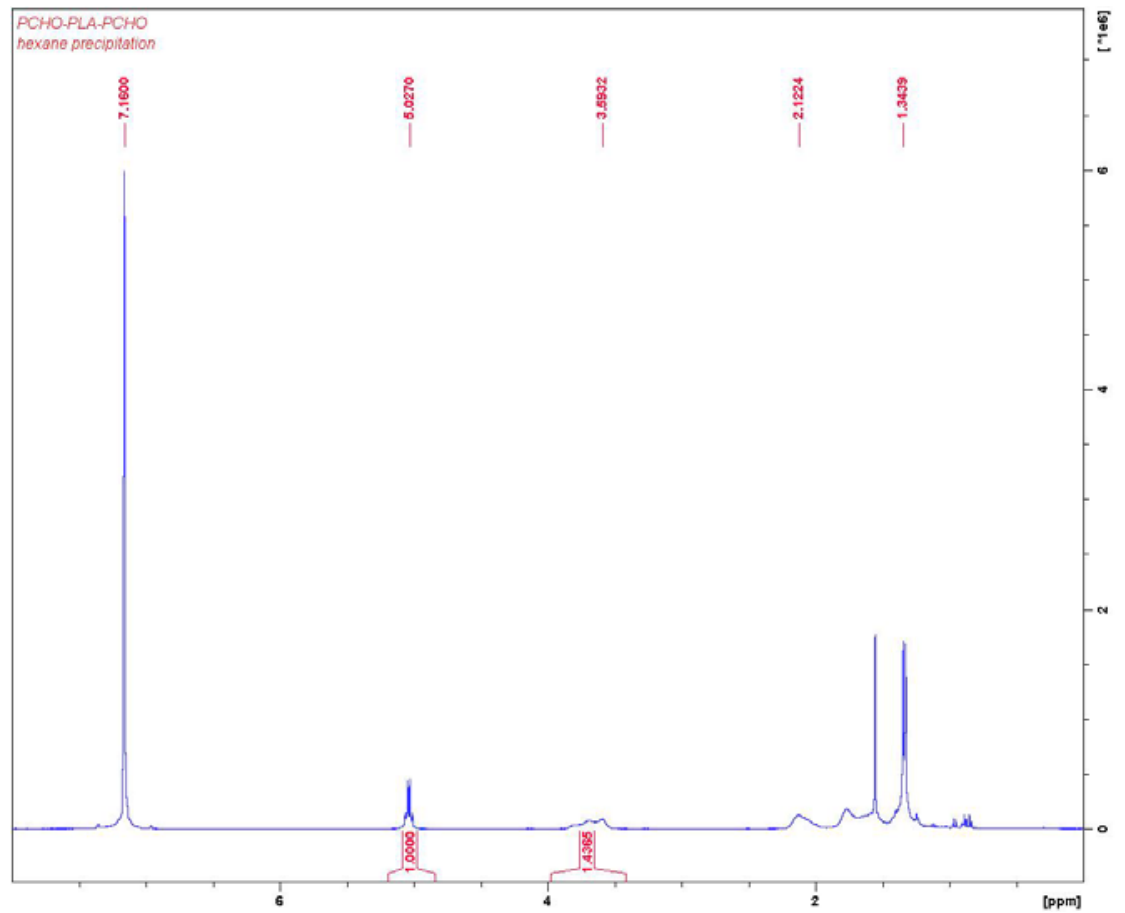

Figure S82. Table S5, entry 5. Hexanes precipitate from selective precipitation of PCHO-PLAPCHO. ${ }^{1} \mathrm{H}$ NMR (300 MHz, $\left.25{ }^{\circ} \mathrm{C}, \mathrm{C}_{6} \mathrm{D}_{6}\right), \delta$ (ppm): 5.03 (q, 2H, $\left.\mathrm{CHCH}_{3} \mathrm{PLA}\right), 3.59$ (m, 2H, $\mathrm{COCH} \mathrm{PCHO}$ ), 2.12 (m, 2H, $\left.\mathrm{COCHCH}_{2} \mathrm{PCHO}\right), 1.35$ (d, 6H, $\left.\mathrm{CHCH}_{3} \mathrm{PLA}\right)$. 


\section{Low weight copolymer study}

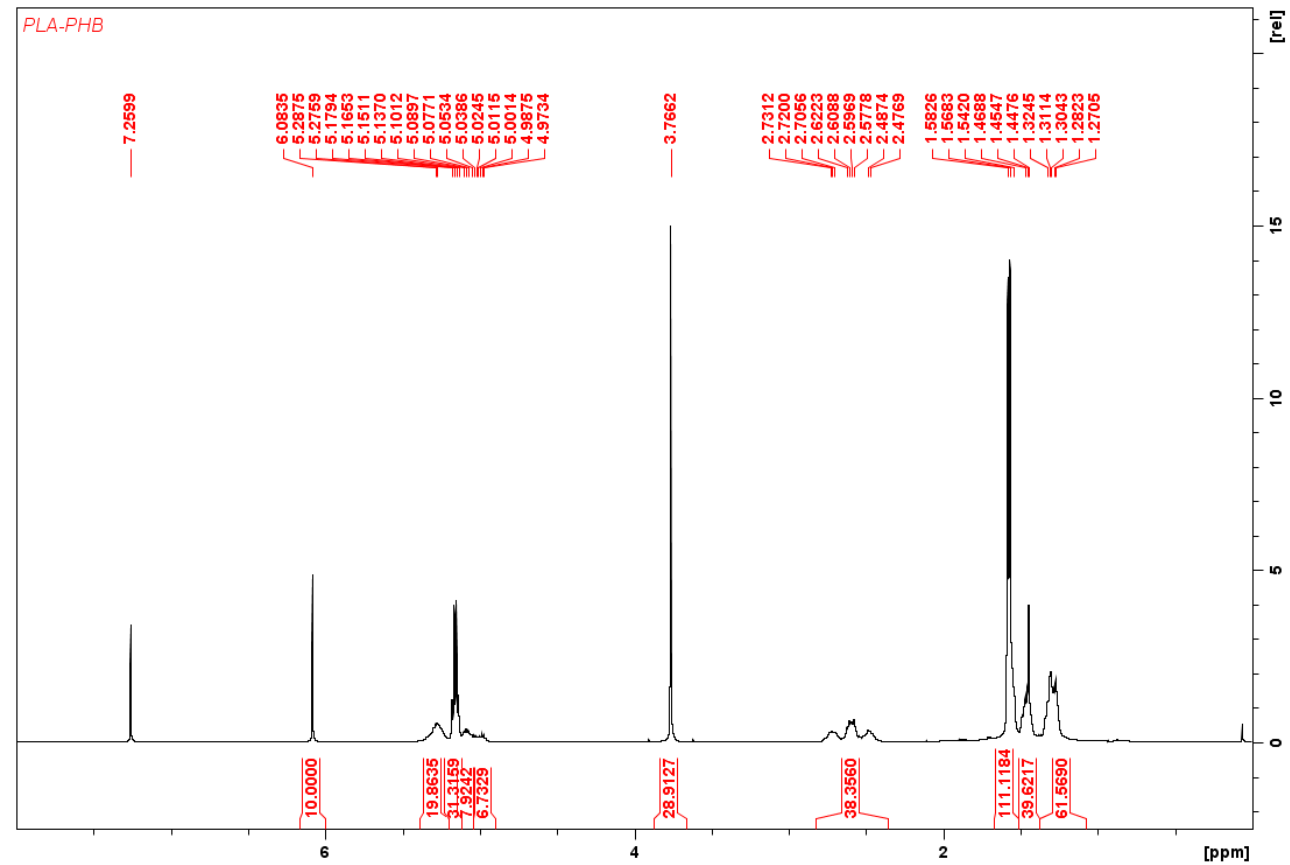

Figure S83. Table 5, entry 1. Low molecular weight PLA-PHB copolymer. ${ }^{1} \mathrm{H}$ NMR (500 MHz, $\left.25^{\circ} \mathrm{C}, \mathrm{CDCl}_{3}\right), \delta(\mathrm{ppm}): 6.08(\mathrm{~s}, 3 \mathrm{H}, \mathrm{PhH} \mathrm{TMB}), 5.27\left(\mathrm{~m}, 1 \mathrm{H}, \mathrm{OCHCH}_{3} \mathrm{PHB}\right), 5.16-5.00(\mathrm{~m}, 2 \mathrm{H}$, $\mathrm{CHCH}_{3} \mathrm{PLA}$ ), 3.77 (s, 9H, CH3 TMB), 2.60 (m, 2H, $\left.\mathrm{COCH}_{2} \mathrm{PHB}\right), 1.57$ (d, 6H, CHCH 3 PLA), 1.30 (t, 3H, $\left.\mathrm{OCHCH}_{3} \mathrm{PHB}\right)$.

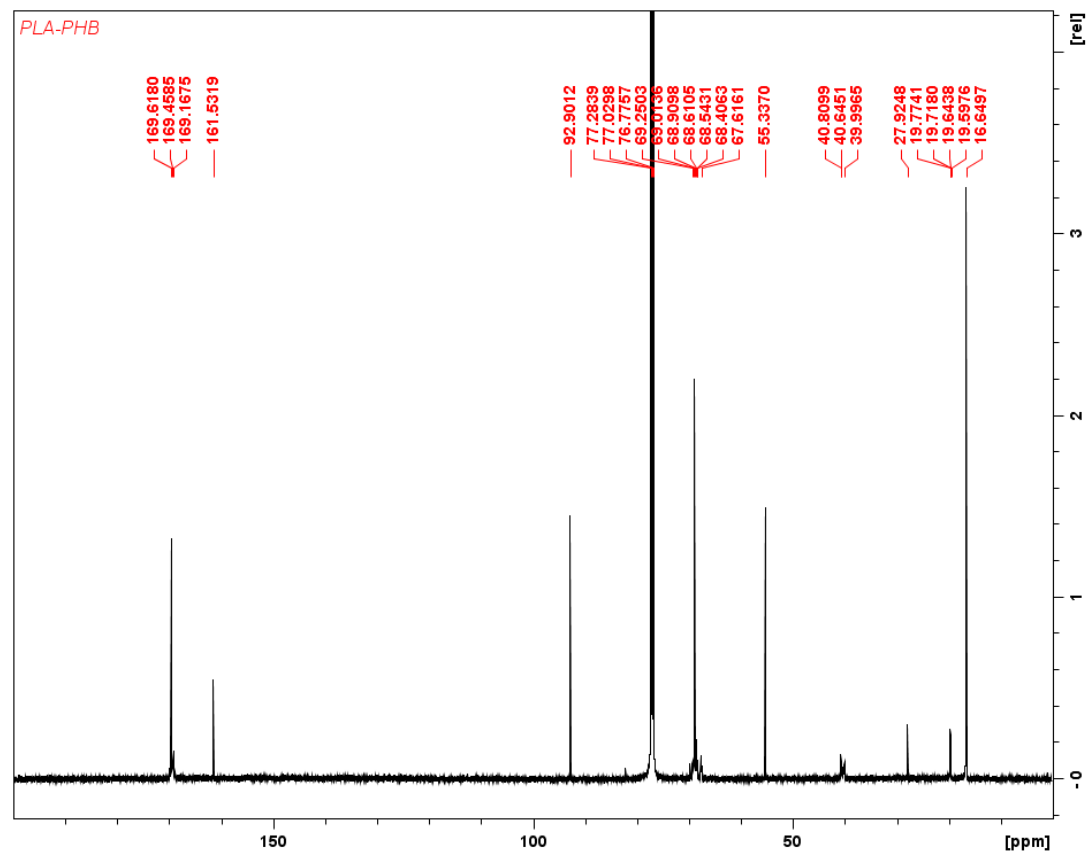

Figure S84. Table 5, entry 1. Low molecular weight PLA-PHB copolymer. ${ }^{13} \mathrm{C}$ NMR $(500 \mathrm{MHz}$, $\left.25{ }^{\circ} \mathrm{C}, \mathrm{CDCl}_{3}\right), \delta$ (ppm): 169.6 ( $\left.C=\mathrm{O}, \mathrm{PLA}\right), 169.2$ ( $\left.C=\mathrm{O}, \mathrm{PHB}\right), 161.5$ (C-OMe, TMB), 92.9 $(\mathrm{CH}, \mathrm{TMB}), 77.0\left(\mathrm{CDCl}_{3}\right), 68.6(\mathrm{CH}, \mathrm{PLA}), 67.6(\mathrm{CH}, \mathrm{PHB}), 55.3\left(\mathrm{OCH}_{3}, \mathrm{TMB}\right), 40.6\left(\mathrm{CH}_{2}\right.$, PHB $), 27.9\left(\mathrm{CH}_{3}, \mathrm{O}^{t} \mathrm{Bu}\right), 19.6\left(\mathrm{CH}_{3}, \mathrm{PHB}\right), 16.6\left(\mathrm{CH}_{3}, \mathrm{PLA}\right)$. 


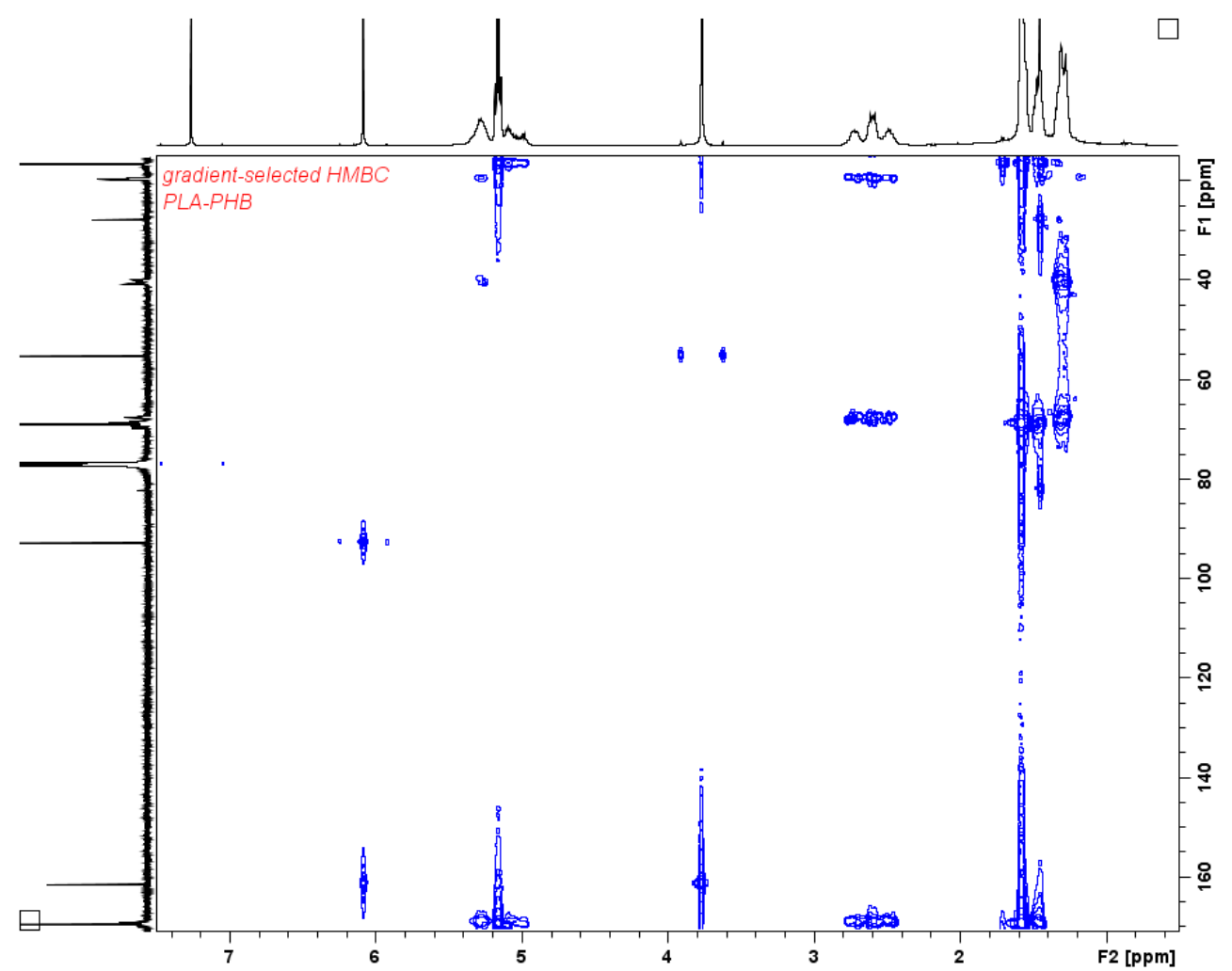

Figure S85. Table 5, entry 1. Low molecular weight PLA-PHB copolymer. HMBC ${ }^{1} \mathrm{H}-{ }^{13} \mathrm{C}$ NMR $\left(500 \mathrm{MHz}, 25^{\circ} \mathrm{C}, \mathrm{CDCl}_{3}\right)$.

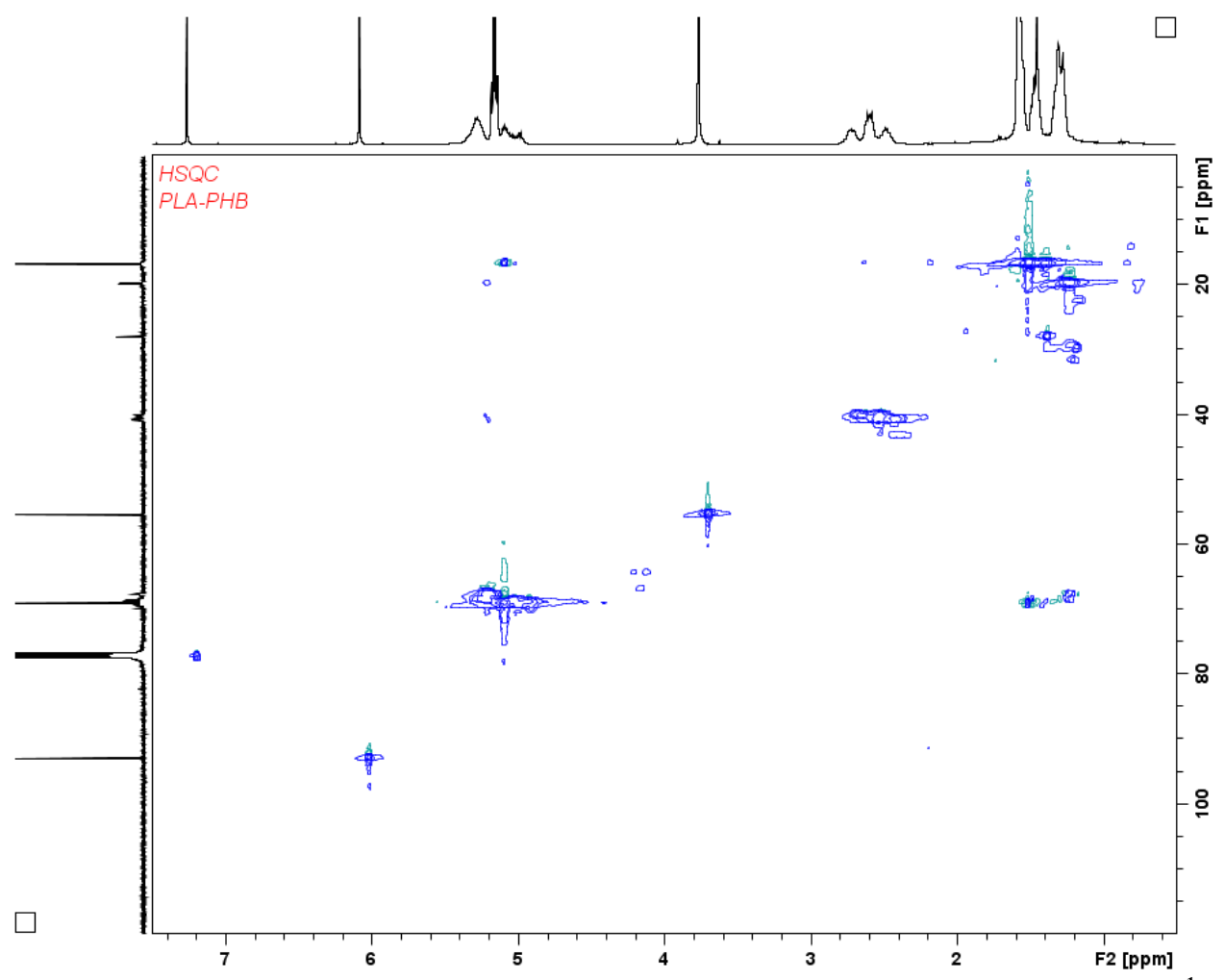

Figure S86. Table 5, entry 1. Low molecular weight PLA-PHB copolymer. HSQC ${ }^{1} \mathrm{H}-{ }^{13} \mathrm{C}$ NMR $\left(500 \mathrm{MHz}, 25^{\circ} \mathrm{C}, \mathrm{CDCl}_{3}\right)$. 


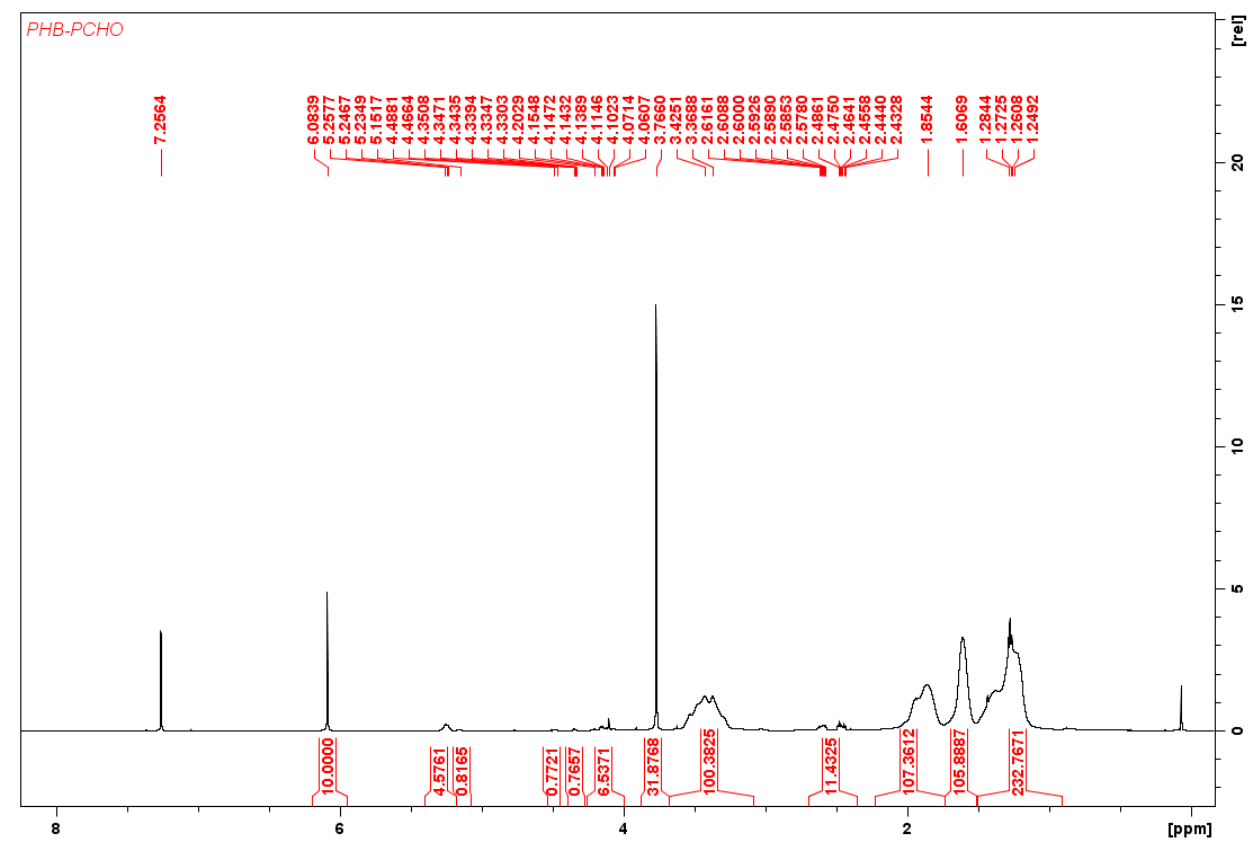

Figure S87. Table 5, entry 2. Low molecular weight PHB-PCHO. ${ }^{1} \mathrm{H}$ NMR $\left(500 \mathrm{MHz}, 25{ }^{\circ} \mathrm{C}\right.$, $\left.\mathrm{CDCl}_{3}\right), \delta(\mathrm{ppm}): 6.08$ (s, 3H, PhH TMB), 5.25 (m, 1H, $\left.\mathrm{OCHCH}_{3} \mathrm{PHB}\right), 3.77$ (s, 9H, $\mathrm{CH}_{3}$ TMB), $3.39(\mathrm{~m}, 2 \mathrm{H}, \mathrm{COCH} \mathrm{PCHO}), 2.53\left(\mathrm{~m}, 2 \mathrm{H}, \mathrm{COCH}_{2} \mathrm{PHB}\right), 1.85\left(\mathrm{~m}, 2 \mathrm{H}, \mathrm{COCHCH}_{2}\right.$ $\mathrm{PCHO}), 1.61\left(\mathrm{~m}, 2 \mathrm{H}, \mathrm{COCHCH}_{2} \mathrm{PCHO}\right), 1.30\left(\mathrm{t}, 3 \mathrm{H}, \mathrm{OCHCH}_{3} \mathrm{PHB}\right), 1.26(\mathrm{~m}, 2 \mathrm{H}$, $\mathrm{COCHCH}_{2} \mathrm{CH}_{2} \mathrm{PCHO}$ ).

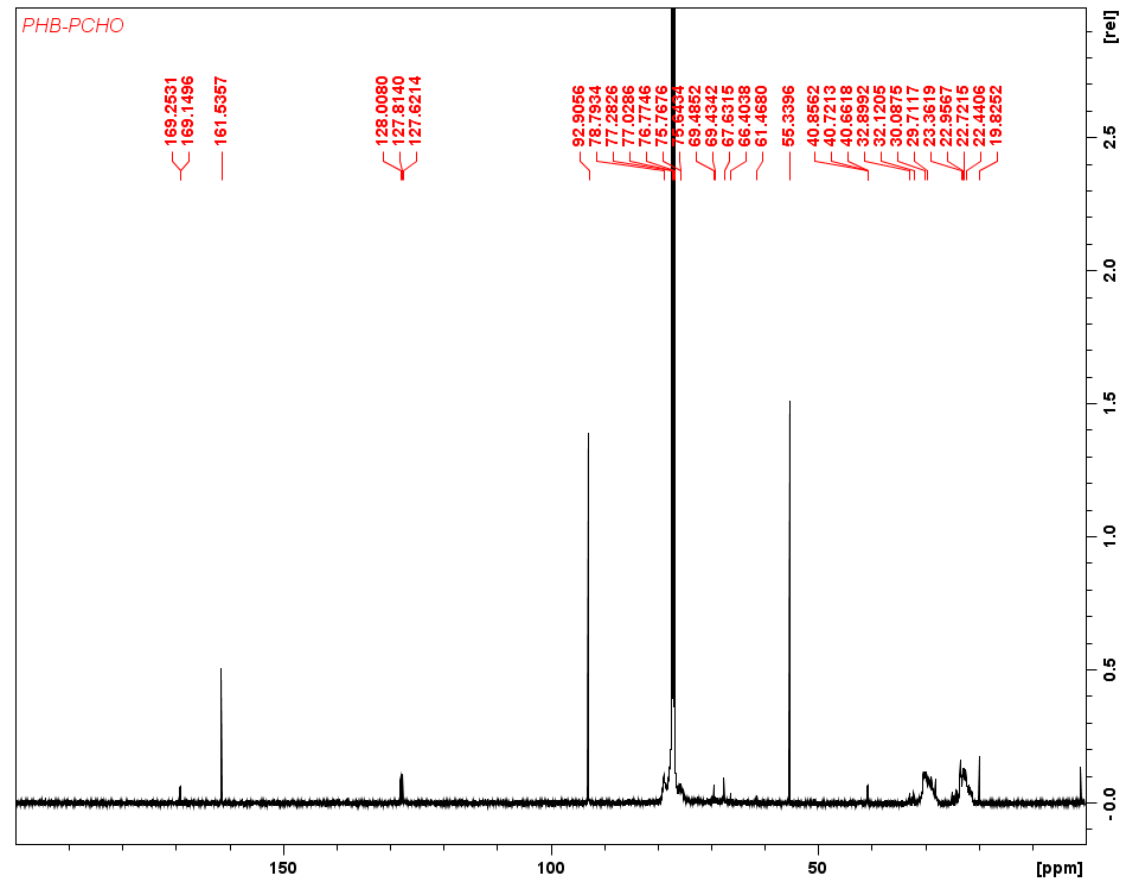

Figure S88. Table 5, entry 2. Low molecular weight PHB-PCHO. ${ }^{13} \mathrm{C}$ NMR $\left(500 \mathrm{MHz}, 25{ }^{\circ} \mathrm{C}\right.$, $\mathrm{CDCl}_{3}$ ), $\delta$ (ppm): $169.2(C=\mathrm{O}, \mathrm{PHB}), 161.5$ (C-OMe, TMB), 127.8 (residual $\left.\mathrm{C}_{6} \mathrm{D}_{6}\right), 92.9(\mathrm{CH}$, TMB), 77.0 (broad, $\mathrm{CH}, \mathrm{PCHO}), 77.0\left(\mathrm{CDCl}_{3}\right), 67.6(\mathrm{CH}, \mathrm{PHB}), 55.3\left(\mathrm{OCH}_{3}, \mathrm{TMB}\right), 40.6\left(\mathrm{CH}_{2}\right.$, PHB), 32.1 (broad, $\left.\mathrm{CHCH}_{2}, \mathrm{PCHO}\right), 27.9\left(\mathrm{CH}_{3}, \mathrm{O}^{\mathrm{t}} \mathrm{Bu}\right), 22.7$ (broad, $\left.\mathrm{CH}_{2}, \mathrm{PCHO}\right), 19.8\left(\mathrm{CH}_{3}\right.$, PHB). 


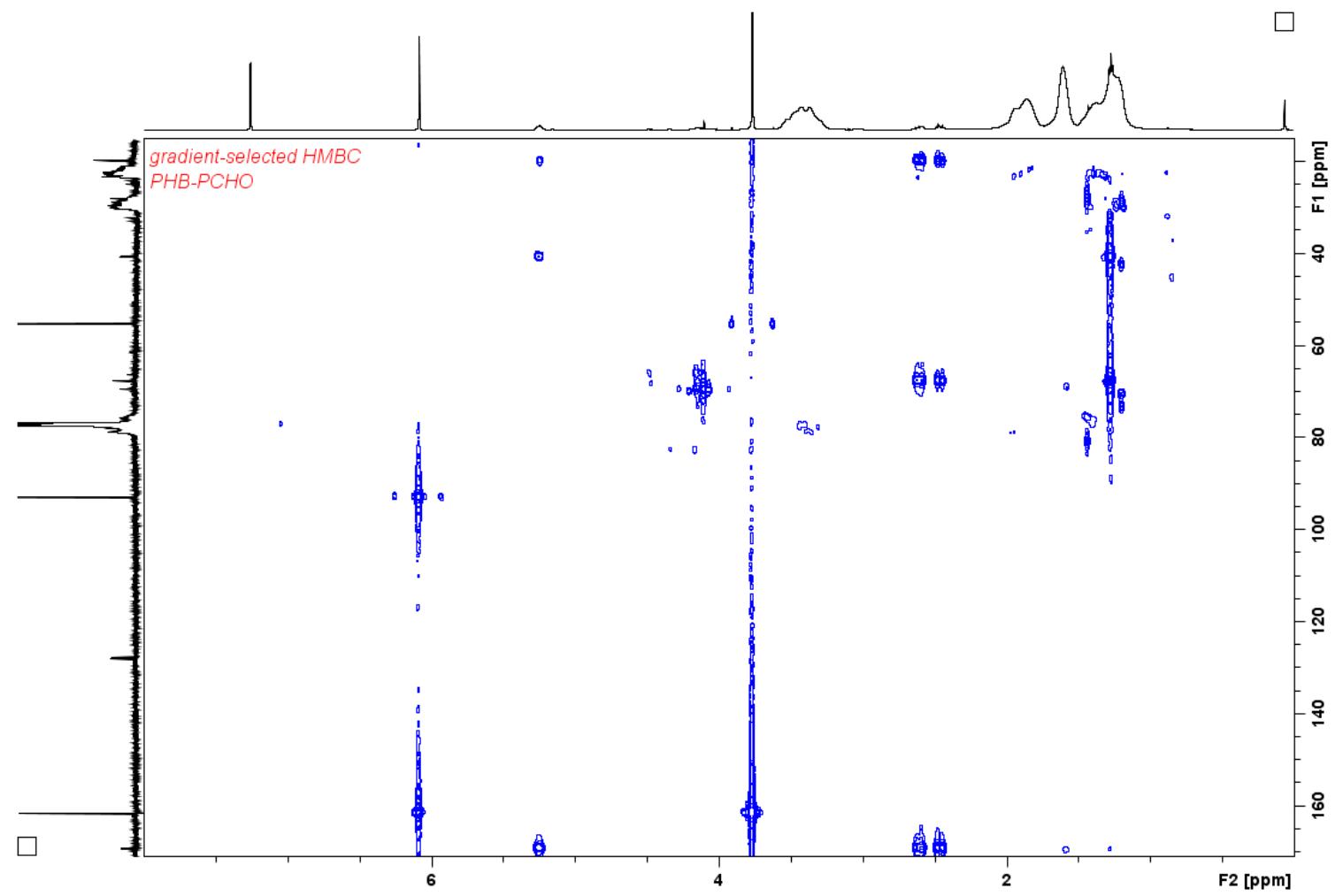

Figure S89. Table 5, entry 2. Low molecular weight PHB-PCHO. $\mathrm{HMBC}{ }^{1} \mathrm{H}^{13} \mathrm{C}$ NMR (500 $\left.\mathrm{MHz}, 25{ }^{\circ} \mathrm{C}, \mathrm{CDCl}_{3}\right)$.

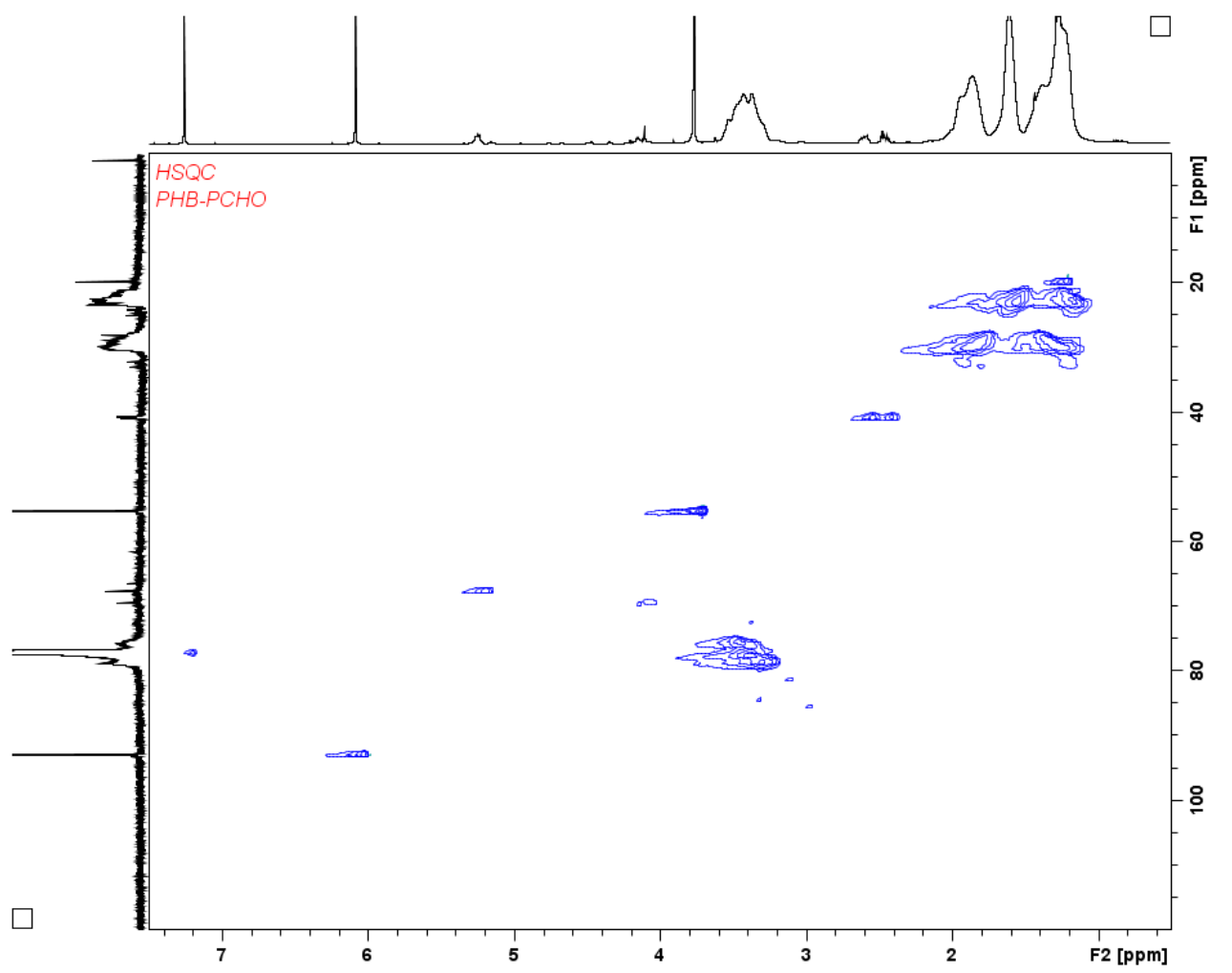

Figure S90. Table 5, entry 2. Low molecular weight PHB-PCHO. HSQC ${ }^{1} \mathrm{H}_{-}{ }^{13} \mathrm{C}$ NMR (500 $\left.\mathrm{MHz}, 25^{\circ} \mathrm{C}, \mathrm{CDCl}_{3}\right)$. 


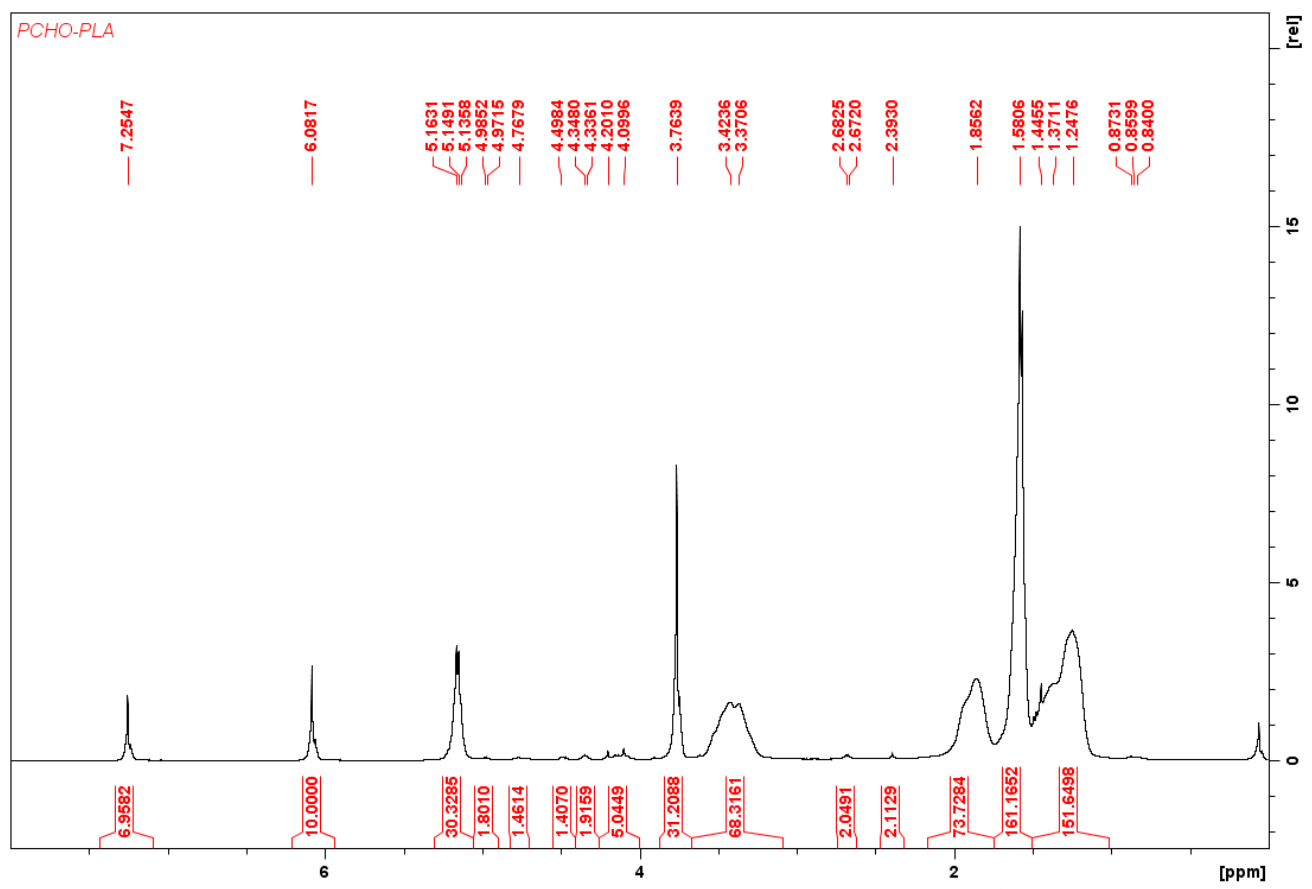

Figure S91. Table 5, entry 3. Low molecular weight PCHO-PLA. ${ }^{1} \mathrm{H}$ NMR $\left(500 \mathrm{MHz}, 25{ }^{\circ} \mathrm{C}\right.$, $\mathrm{CDCl}_{3}$ ), $\delta$ (ppm): 6.08 (s, 3H, PhH TMB), 5.15 (q, 2H, $\left.\mathrm{CHCH}_{3} \mathrm{PLA}\right), 3.77$ (s, 9H, $\mathrm{CH}_{3} \mathrm{TMB}$ ), 3.40 (m, 2H, COCH PCHO), 1.86 (m, 2H, $\left.\mathrm{COCHCH}_{2} \mathrm{PCHO}\right), 1.58$ (d, 6H, $\left.\mathrm{CHCH}_{3} \mathrm{PLA}\right), 1.25$ (m, $2 \mathrm{H}, \mathrm{COCHCH}_{2} \mathrm{CH}_{2} \mathrm{PCHO}$ ).

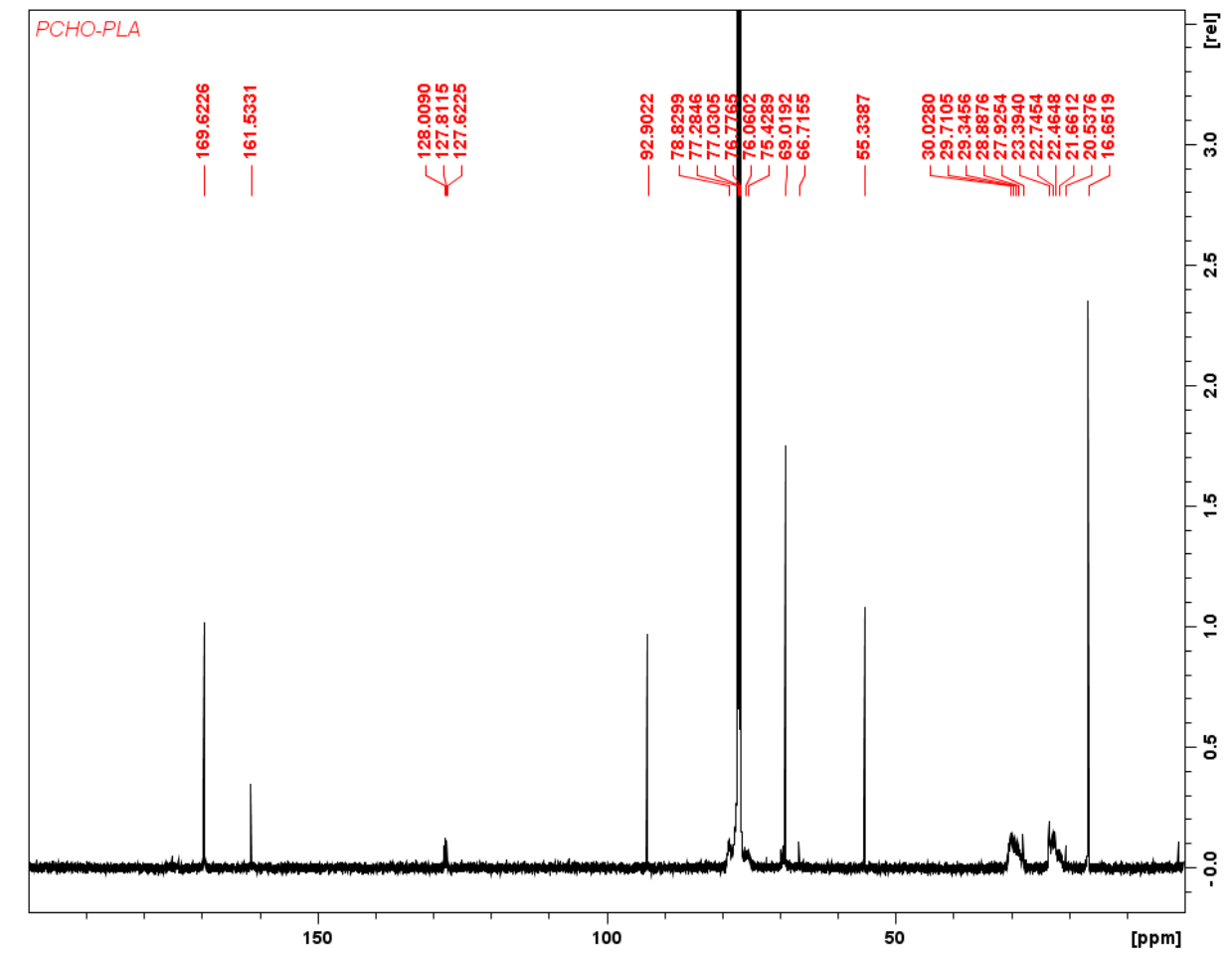

Figure S92. Table 5, entry 3. Low molecular weight PCHO-PLA. ${ }^{13} \mathrm{C} \mathrm{NMR}\left(500 \mathrm{MHz}, 25{ }^{\circ} \mathrm{C}\right.$, $\left.\mathrm{CDCl}_{3}\right), \delta(\mathrm{ppm}): 169.6(C=\mathrm{O}, \mathrm{PLA}), 161.5(C-\mathrm{OMe}, \mathrm{TMB}), 127.8$ (residual $\left.\mathrm{C}_{6} \mathrm{D}_{6}\right), 92.9(\mathrm{CH}$, TMB), 77.0 (broad, $\mathrm{CH}, \mathrm{PCHO}), 77.0\left(\mathrm{CDCl}_{3}\right), 68.6(\mathrm{CH}, \mathrm{PLA}), 55.3\left(\mathrm{OCH}_{3}, \mathrm{TMB}\right), 29.3$ (broad, $\left.\mathrm{CHCH}_{2}, \mathrm{PCHO}\right), 27.9\left(\mathrm{CH}_{3}, \mathrm{O}^{t} \mathrm{Bu}\right), 22.7$ (broad, $\left.\mathrm{CH}_{2}, \mathrm{PCHO}\right), 16.6\left(\mathrm{CH}_{3}, \mathrm{PLA}\right)$. 


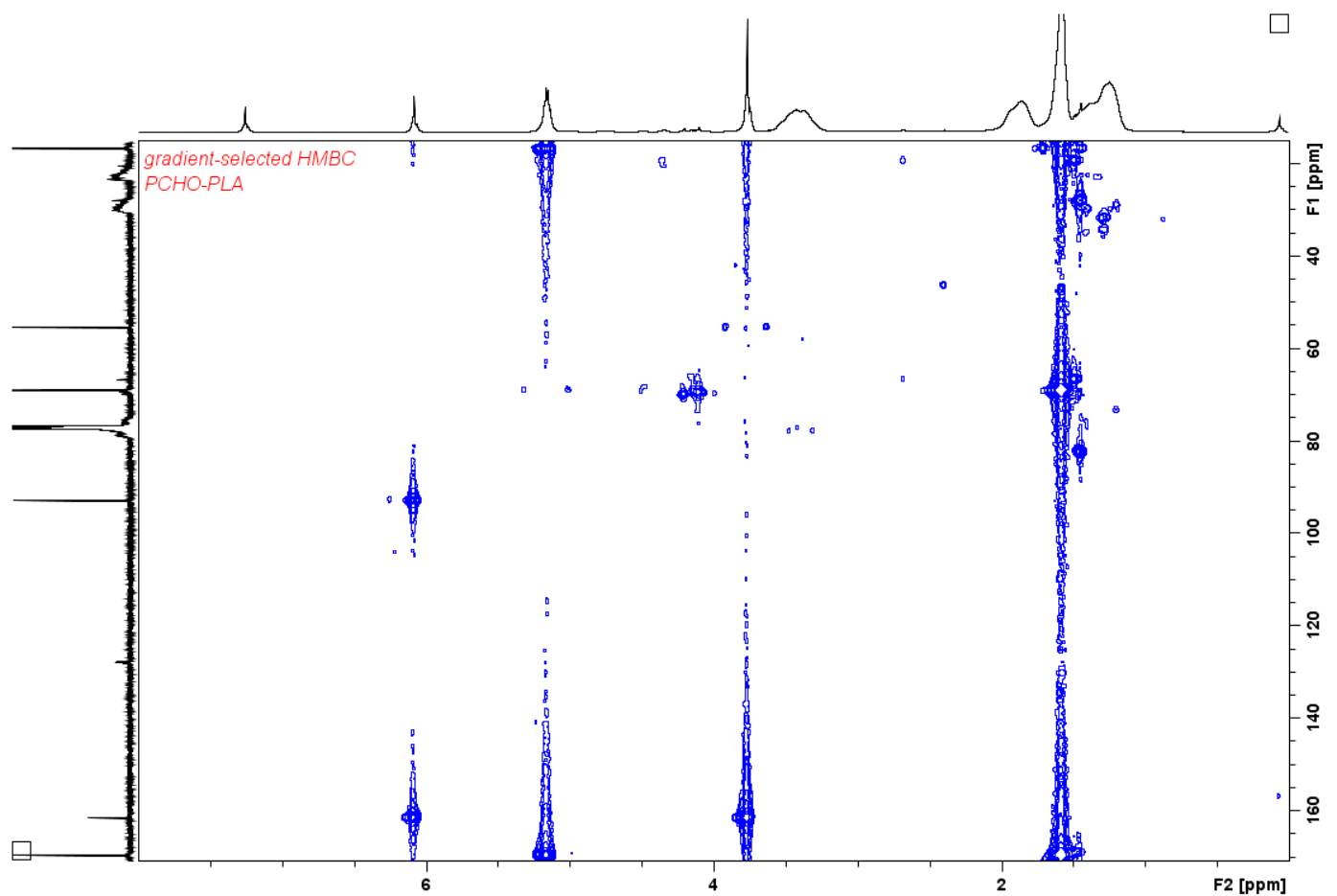

Figure S93. Table 5, entry 3. Low molecular weight PCHO-PLA. HMBC ${ }^{1} \mathrm{H}^{13}{ }^{13} \mathrm{C}$ NMR $(500$ $\left.\mathrm{MHz}, 25{ }^{\circ} \mathrm{C}, \mathrm{CDCl}_{3}\right)$.

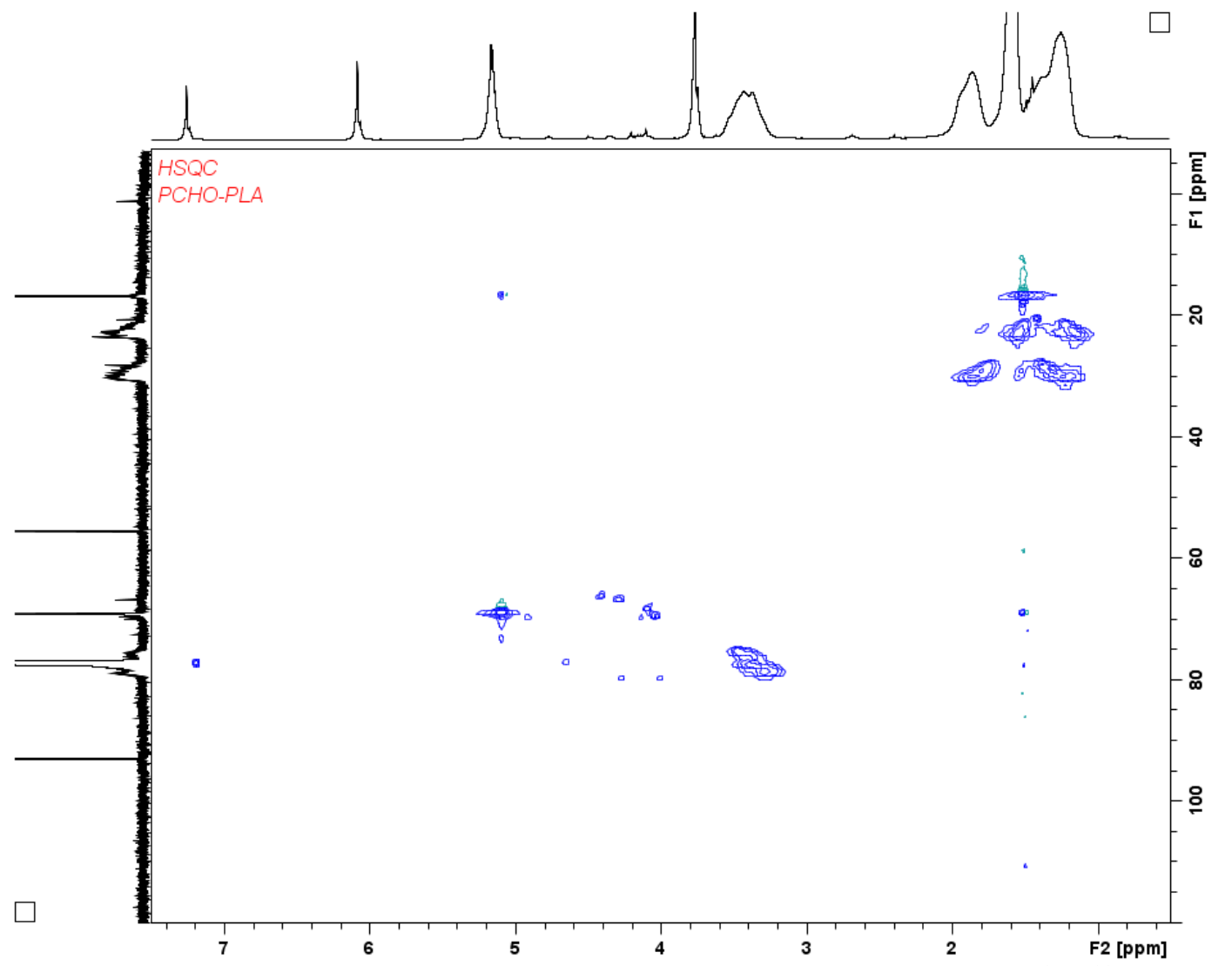

Figure S94. Table 5, entry 3. Low molecular weight PCHO-PLA. HSQC ${ }^{1} \mathrm{H}-{ }^{13} \mathrm{C}$ NMR (500 $\left.\mathrm{MHz}, 25^{\circ} \mathrm{C}, \mathrm{CDCl}_{3}\right)$. 


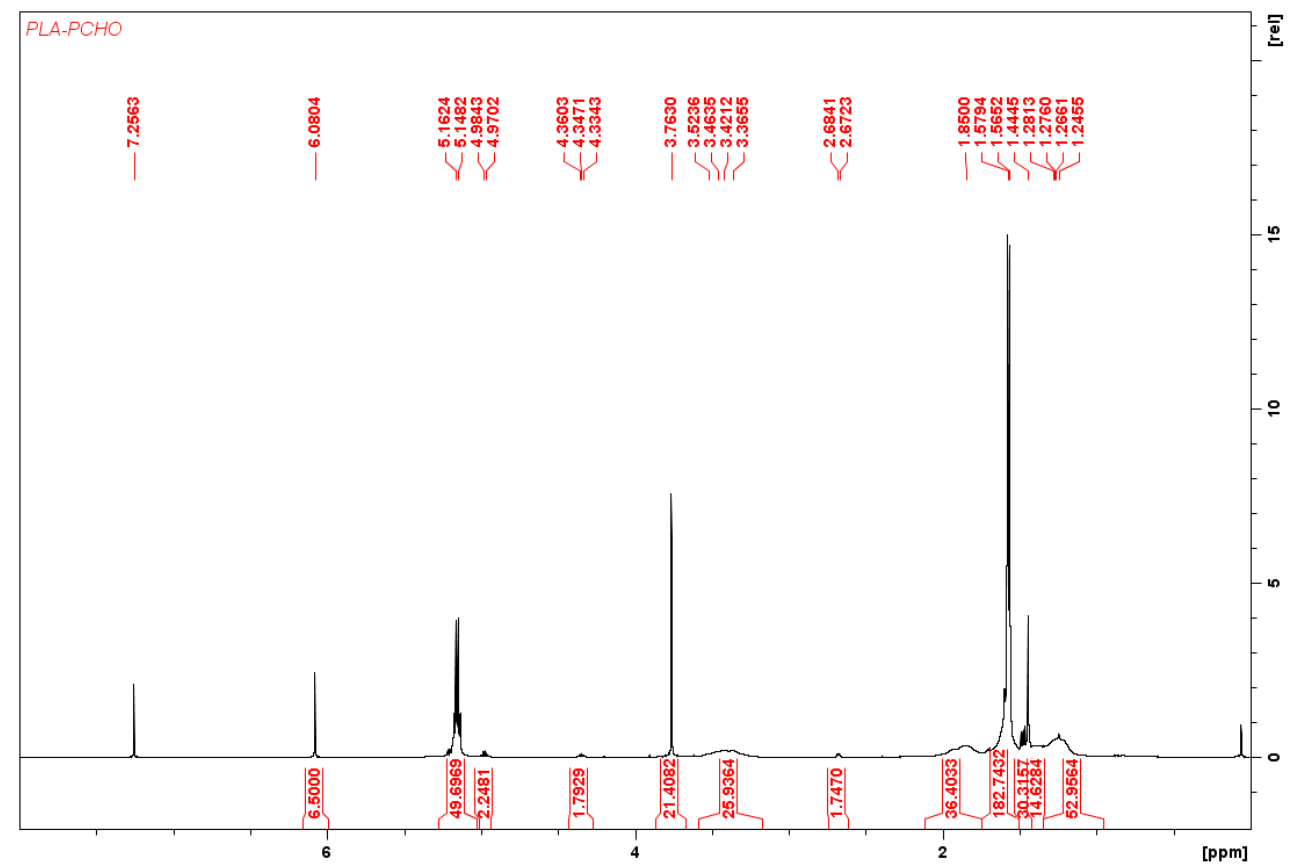

Figure S95. Table 5, entry 4. Low molecular weight PLA-PCHO. ${ }^{1} \mathrm{H}$ NMR $\left(500 \mathrm{MHz}, 25{ }^{\circ} \mathrm{C}\right.$, $\mathrm{CDCl}_{3}$ ), $\delta$ (ppm): 6.08 (s, 3H, PhH TMB), 5.15 (q, 2H, $\left.\mathrm{CHCH}_{3} \mathrm{PLA}\right), 3.77$ (s, 9H, $\mathrm{CH}_{3} \mathrm{TMB}$ ), $3.40(\mathrm{~m}, 2 \mathrm{H}, \mathrm{COCH} \mathrm{PCHO}), 1.86\left(\mathrm{~m}, 2 \mathrm{H}, \mathrm{COCHCH}_{2} \mathrm{PCHO}\right), 1.58$ (d, 6H, $\left.\mathrm{CHCH}_{3} \mathrm{PLA}\right), 1.25$ (m, $\left.2 \mathrm{H}, \mathrm{COCHCH}_{2} \mathrm{CH}_{2} \mathrm{PCHO}\right)$.

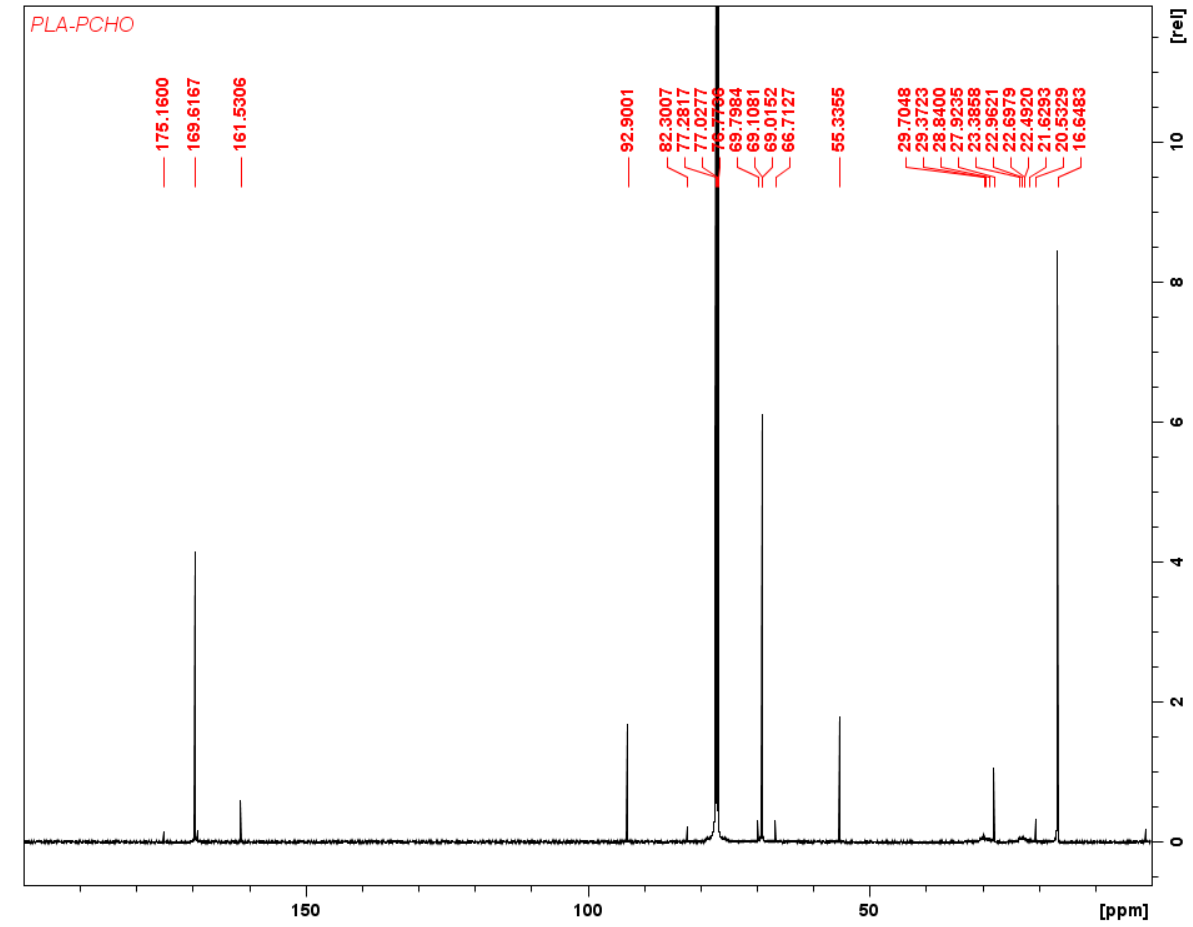

Figure S96. Table 5, entry 4. Low molecular weight PLA-PCHO. ${ }^{13} \mathrm{C} \mathrm{NMR}\left(500 \mathrm{MHz}, 25{ }^{\circ} \mathrm{C}\right.$, $\left.\mathrm{CDCl}_{3}\right), \delta$ (ppm): 175.1 ( $\left.C=\mathrm{O}, \mathrm{PLA}\right), 169.6$ ( $\left.C=\mathrm{O}, \mathrm{PLA}\right), 161.5$ ( $\left.C=\mathrm{O}, \mathrm{PLA}\right), 161.5$ ( $C-\mathrm{OMe}$, TMB), $92.9(\mathrm{CH}, \mathrm{TMB}), 82.3\left(\mathrm{C}\left(\mathrm{CH}_{3}\right)_{3}, \mathrm{O}^{\mathrm{t}} \mathrm{Bu}\right), 77.0$ (broad, $\left.\mathrm{CH}, \mathrm{PCHO}\right), 77.0\left(\mathrm{CDCl}_{3}\right), 68.6$ ( $\mathrm{CH}, \mathrm{PLA}), 55.3\left(\mathrm{OCH}_{3}, \mathrm{TMB}\right), 29.3$ (broad, $\left.\mathrm{CHCH}_{2}, \mathrm{PCHO}\right), 27.9\left(\mathrm{CH}_{3}, \mathrm{O}^{t} \mathrm{Bu}\right), 22.7$ (broad, $\left.\mathrm{CH}_{2}, \mathrm{PCHO}\right), 16.6\left(\mathrm{CH}_{3}, \mathrm{PLA}\right)$. 


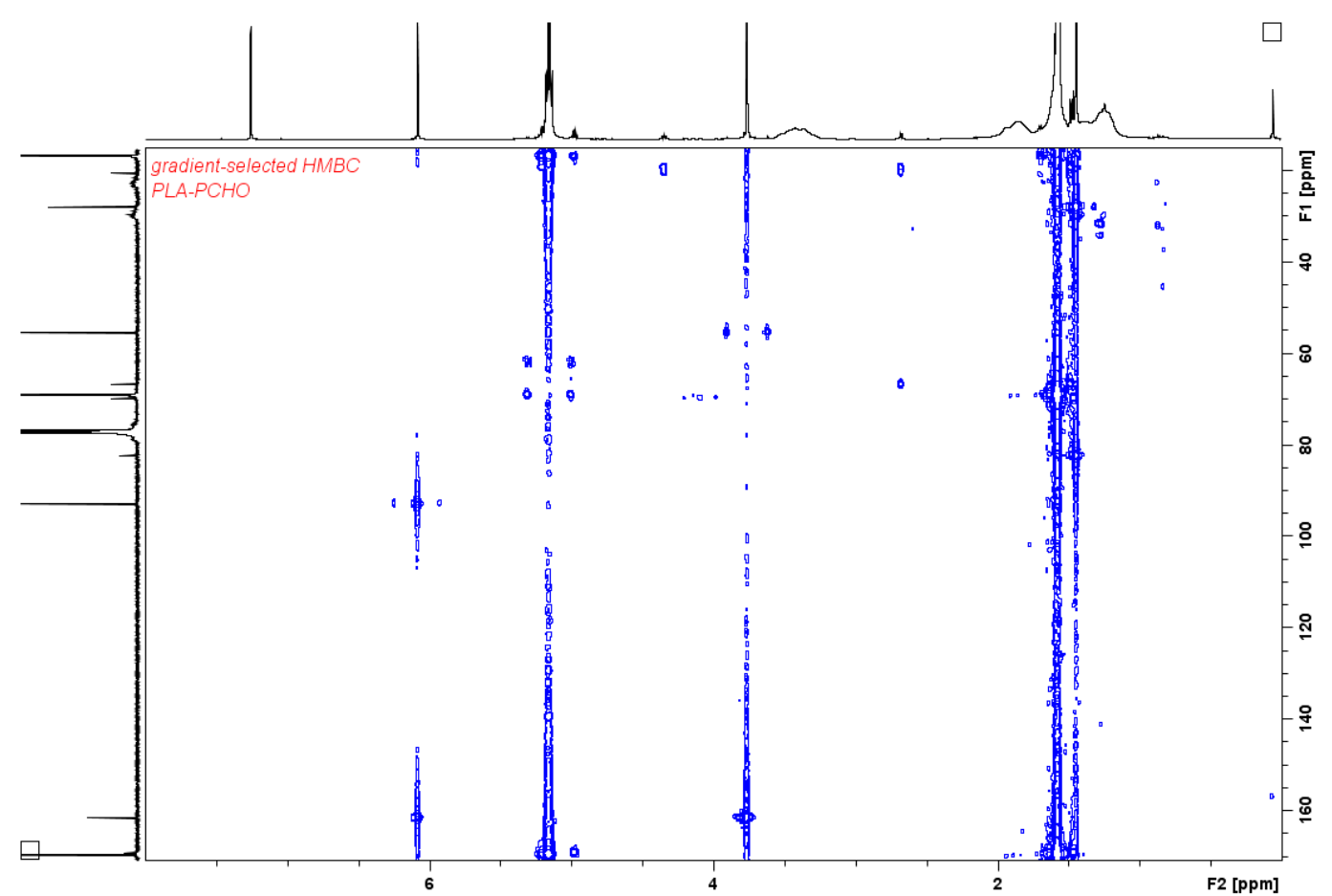

Figure S97. Table 5, entry 4. Low molecular weight PLA-PCHO. $\mathrm{HMBC}{ }^{1} \mathrm{H}^{13}{ }^{13} \mathrm{C}$ NMR (500 $\left.\mathrm{MHz}, 25{ }^{\circ} \mathrm{C}, \mathrm{CDCl}_{3}\right)$.

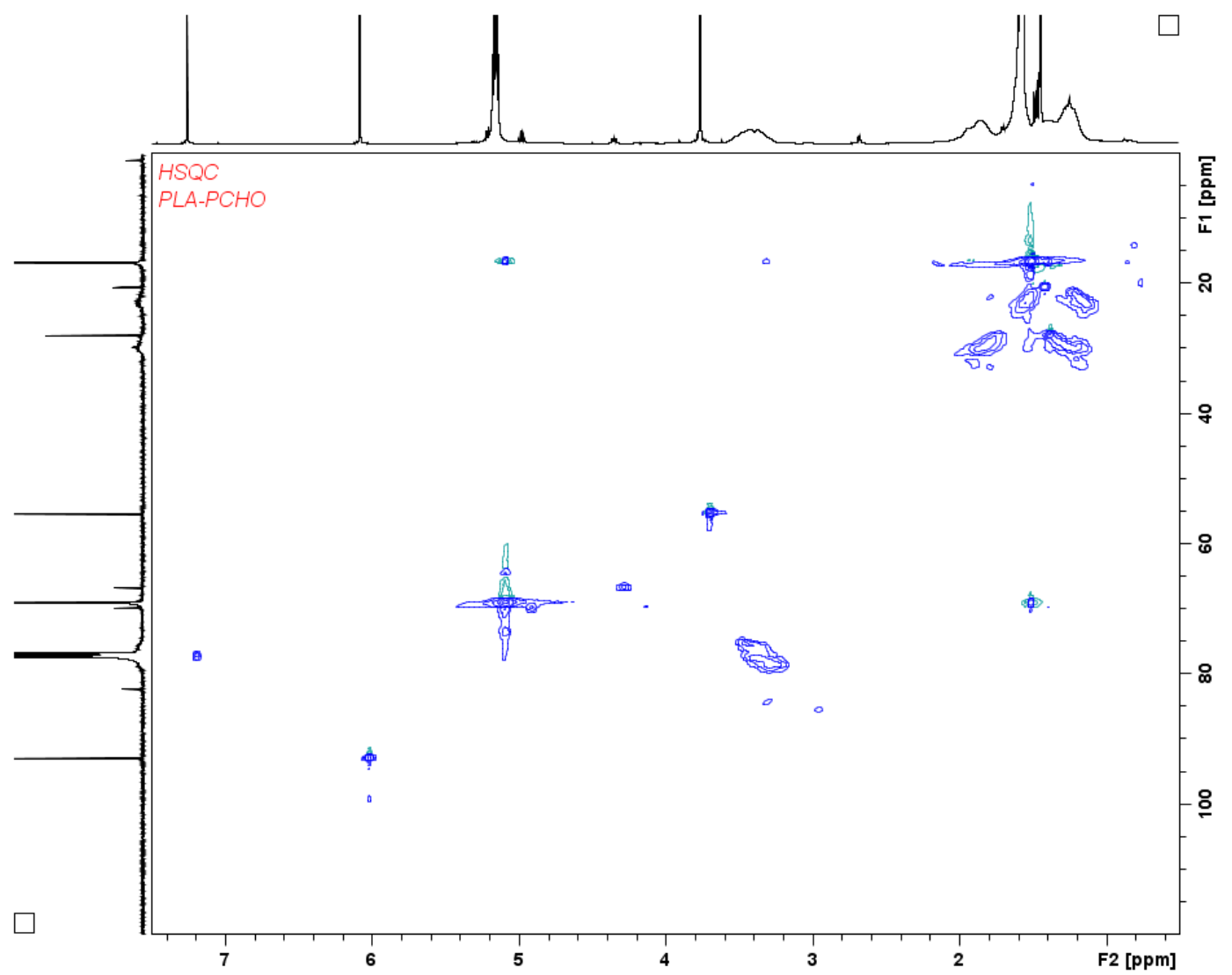

Figure S98. Table 5, entry 4. Low molecular weight PLA-PCHO. HSQC ${ }^{1} \mathrm{H}^{-13} \mathrm{C}$ NMR (500 $\left.\mathrm{MHz}, 25^{\circ} \mathrm{C}, \mathrm{CDCl}_{3}\right)$. 


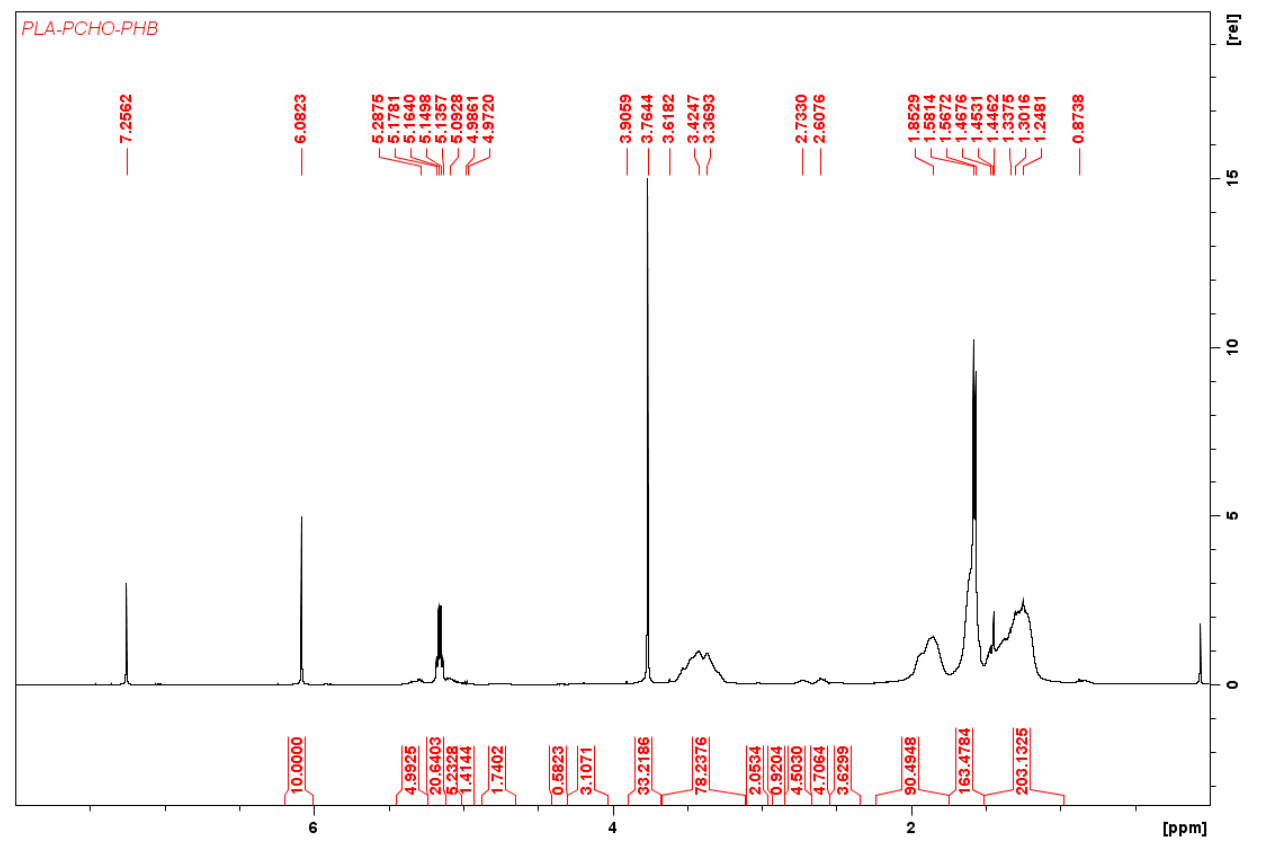

Figure S99. Table 5, entry 5. Low molecular weight PLA-PCHO-PHB. ${ }^{1} \mathrm{H}$ NMR (500 MHz, 25 $\left.{ }^{\circ} \mathrm{C}, \mathrm{CDCl}_{3}\right), \delta(\mathrm{ppm}): 6.08$ (s, 3H, $\mathrm{PhH}$ TMB), 5.29 (m, 1H, $\left.\mathrm{OCHCH}_{3} \mathrm{PHB}\right), 5.15$ (q, 2H, $\mathrm{CHCH}_{3}$ PLA), 3.76 (s, 9H, $\mathrm{CH}_{3} \mathrm{TMB}$ ), 3.40 (m, 2H, COCH PCHO), 2.73-2.61 (m, 2H, $\mathrm{COCH}_{2} \mathrm{PHB}$ ), $1.85\left(\mathrm{~m}, 2 \mathrm{H}, \mathrm{COCHCH}_{2} \mathrm{PCHO}\right), 1.57\left(\mathrm{~d}, 6 \mathrm{H}, \mathrm{CHCH}_{3} \mathrm{PLA}\right), 1.24\left(\mathrm{~m}, 2 \mathrm{H}, \mathrm{COCHCH}_{2} \mathrm{CH}_{2}\right.$ $\mathrm{PCHO})$.

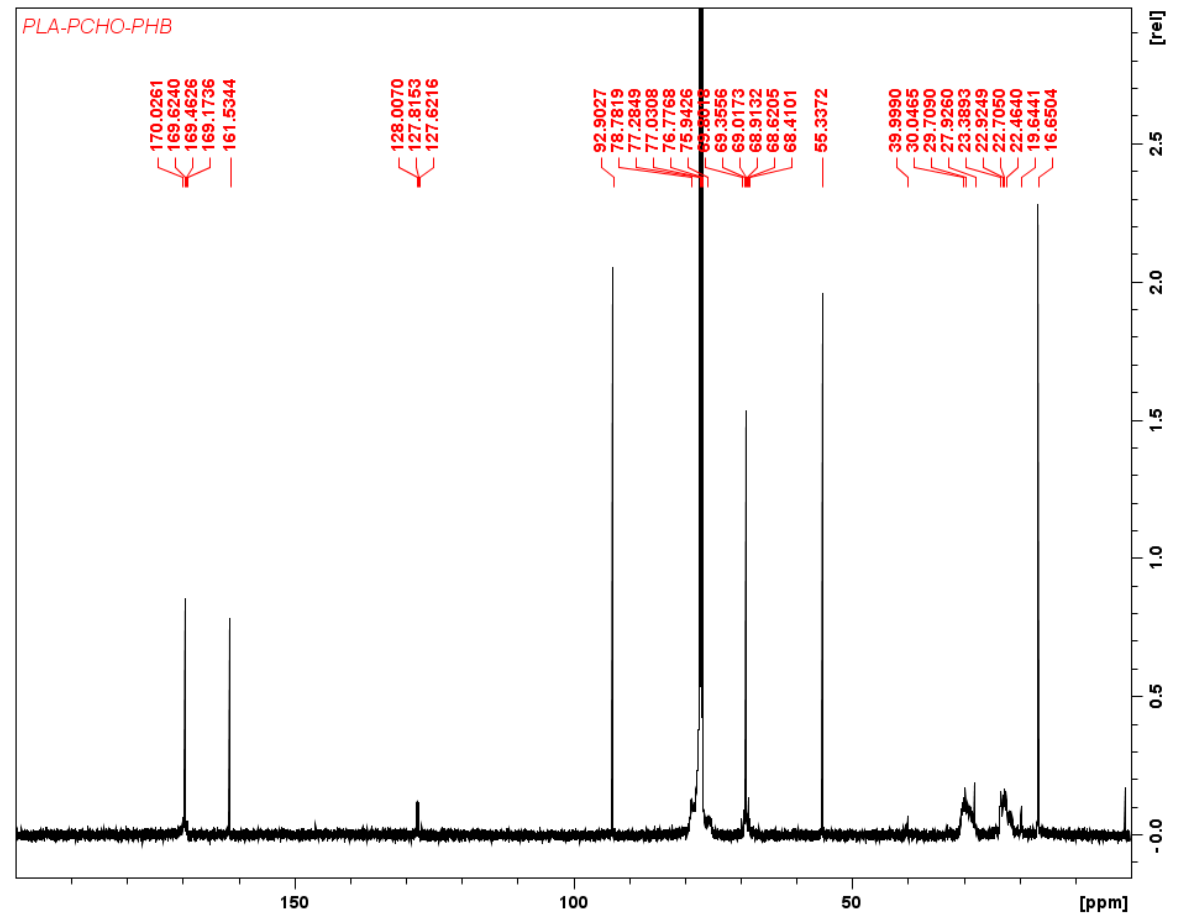

Figure S100. Table 5, entry 5. Low molecular weight PLA-PCHO-PHB. ${ }^{13} \mathrm{C}$ NMR (500 MHz, $\left.25^{\circ} \mathrm{C}, \mathrm{CDCl}_{3}\right), \delta(\mathrm{ppm}): 169.6(\mathrm{C}=\mathrm{O}, \mathrm{PLA}), 169.2$ ( $\left.C=\mathrm{O}, \mathrm{PHB}\right), 161.5$ ( $C$-OMe, TMB), 127.8 (residual $\left.\mathrm{C}_{6} \mathrm{D}_{6}\right), 92.9(\mathrm{CH}, \mathrm{TMB}), 77.0$ (broad, $\left.\mathrm{CH}, \mathrm{PCHO}\right), 77.0\left(\mathrm{CDCl}_{3}\right), 68.6(\mathrm{CH}, \mathrm{PLA}), 67.6$ $(\mathrm{CH}, \mathrm{PHB}), 55.3\left(\mathrm{OCH}_{3}, \mathrm{TMB}\right), 40.0\left(\mathrm{CH}_{2}, \mathrm{PHB}\right), 29.7$ (broad, $\left.\mathrm{CHCH}_{2}, \mathrm{PCHO}\right), 27.9\left(\mathrm{CH}_{3}\right.$, OtBu), 22.7 (broad, $\left.\mathrm{CH}_{2}, \mathrm{PCHO}\right), 19.6\left(\mathrm{CH}_{3}, \mathrm{PHB}\right), 16.6\left(\mathrm{CH}_{3}, \mathrm{PLA}\right)$. 


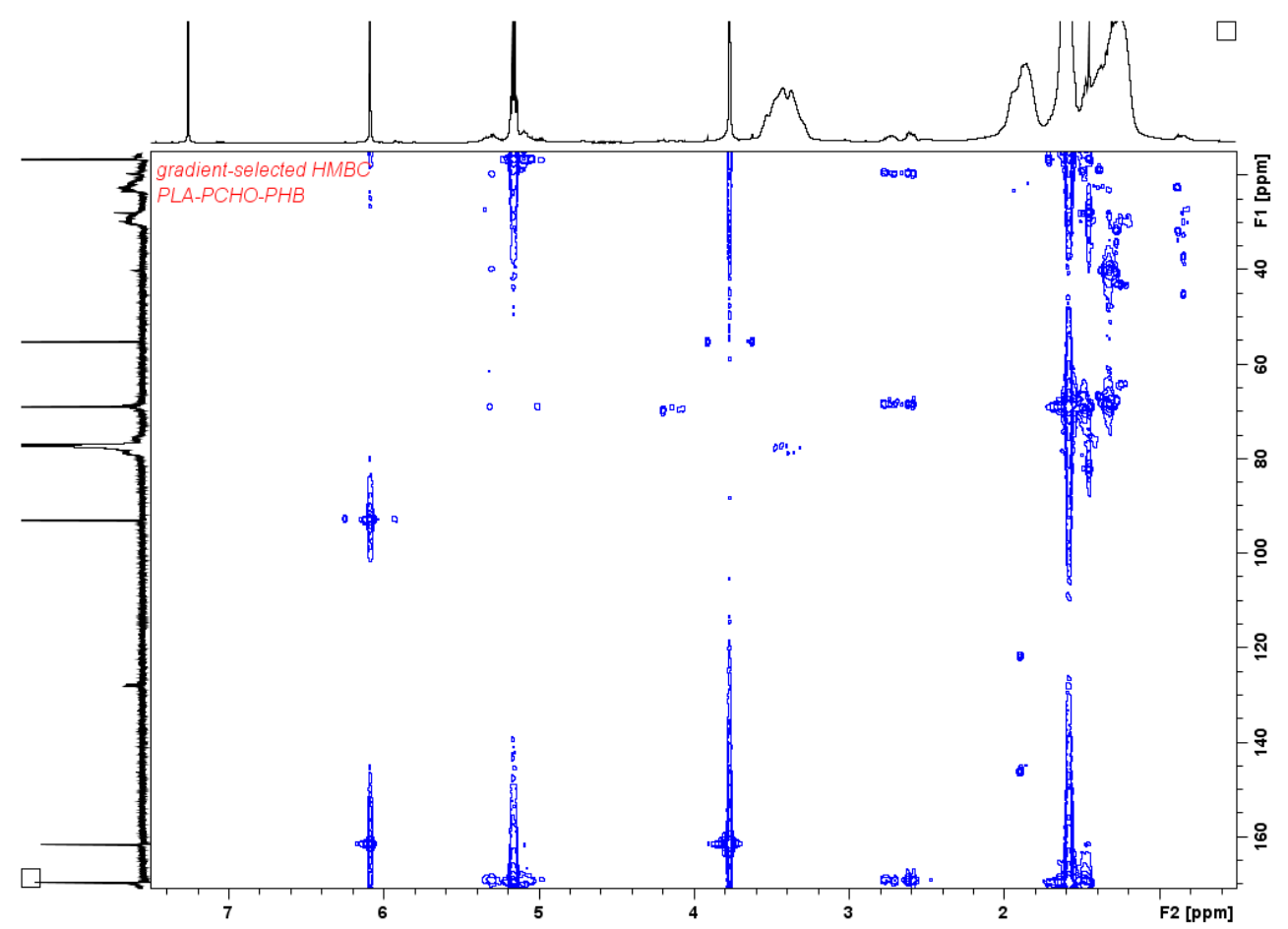

Figure S101. Table 5, entry 5. Low molecular weight PLA-PCHO-PHB. HMBC ${ }^{1} \mathrm{H}_{-}{ }^{13} \mathrm{C}$ NMR $\left(500 \mathrm{MHz}, 25^{\circ} \mathrm{C}, \mathrm{CDCl}_{3}\right)$.

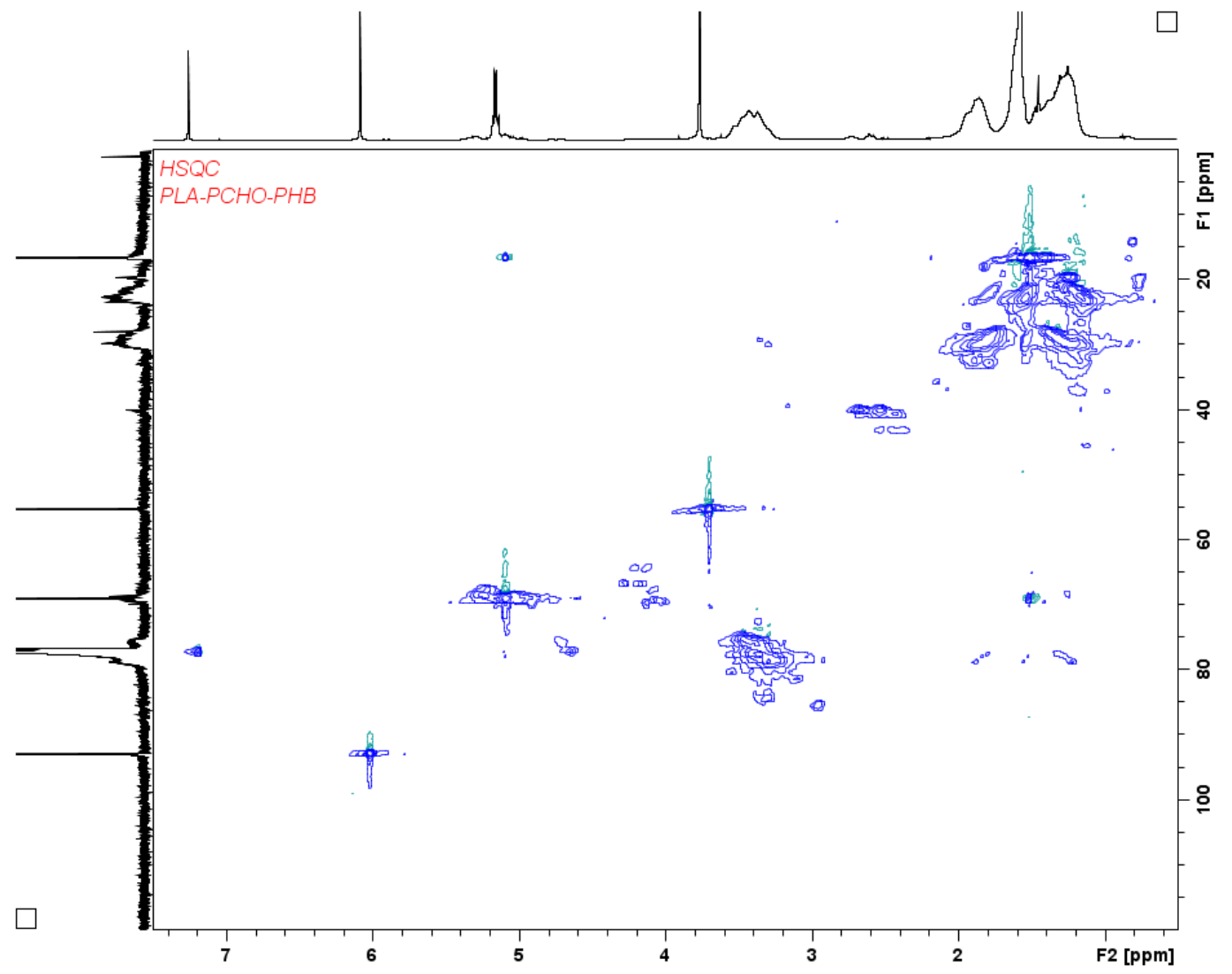

Figure S102. Table 5, entry 5. Low molecular weight PLA-PCHO-PHB. HSQC ${ }^{1} \mathrm{H}-{ }^{13} \mathrm{C}$ NMR $\left(500 \mathrm{MHz}, 25^{\circ} \mathrm{C}, \mathrm{CDCl}_{3}\right)$. 


\section{DOSY Experiments}

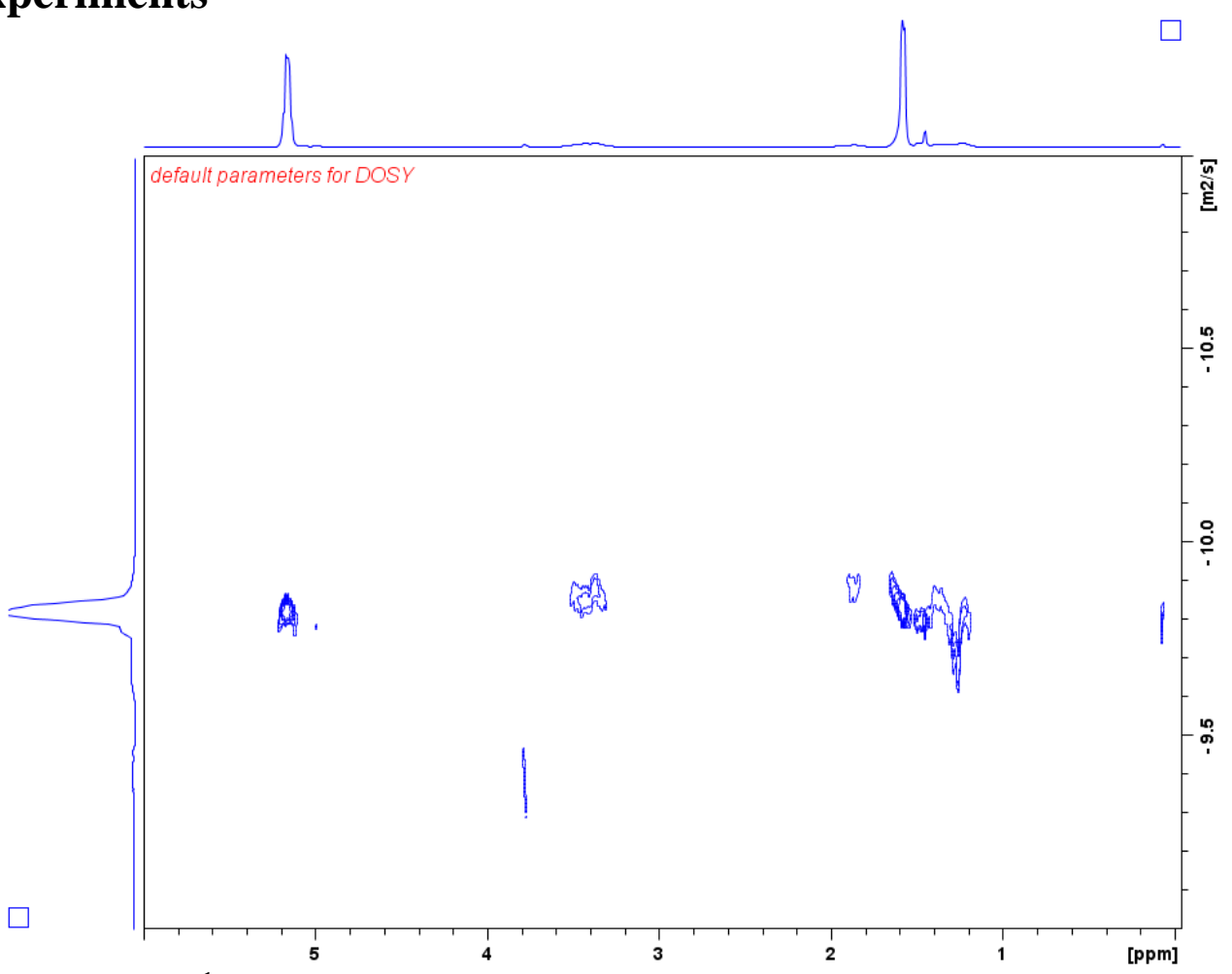

Figure S103. DOSY ${ }^{1} \mathrm{H}$ NMR $\left(500 \mathrm{MHz}, 25{ }^{\circ} \mathrm{C}, \mathrm{C}_{6} \mathrm{D}_{6}\right)$ spectrum of PLA and PCHO homopolymers; $\mathrm{D}=1.53 \times 10^{-10} \mathrm{~m}^{2} \mathrm{~s}^{-1}$ (PLA) and $1.27 \times 10^{-10} \mathrm{~m}^{2} \mathrm{~s}^{-1}$ (PCHO). Residual methanol at $3.8 \mathrm{ppm}$.

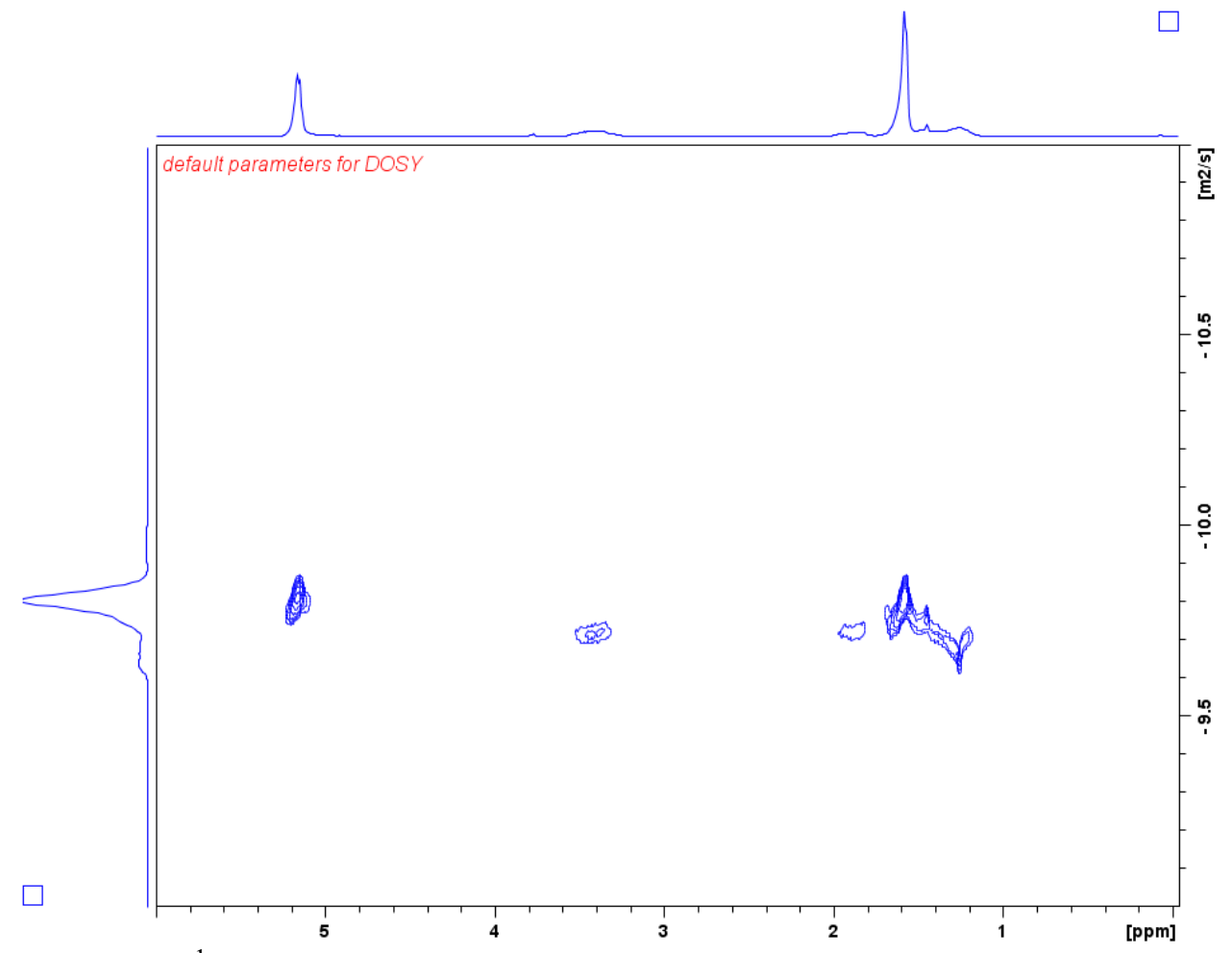

Figure S104. DOSY ${ }^{1} \mathrm{H}$ NMR $\left(500 \mathrm{MHz}, 25{ }^{\circ} \mathrm{C}, \mathrm{C}_{6} \mathrm{D}_{6}\right)$ spectrum of a PLA-PCHO diblock copolymer; $\mathrm{D}=1.76 \times 10^{-10} \mathrm{~m}^{2} \mathrm{~s}^{-1}$. 


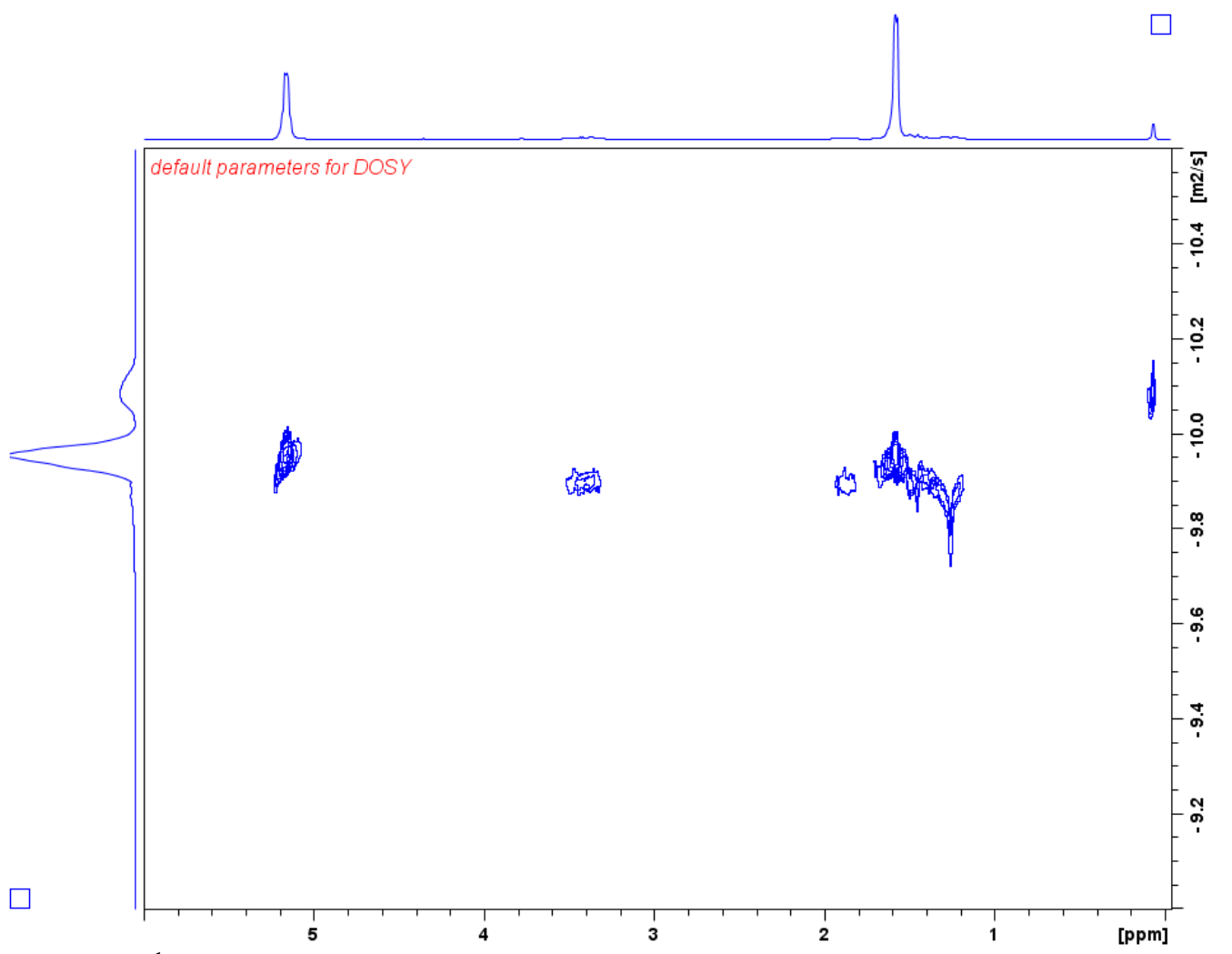

Figure S105. DOSY ${ }^{1} \mathrm{H}$ NMR $\left(500 \mathrm{MHz}, 25^{\circ} \mathrm{C}, \mathrm{C}_{6} \mathrm{D}_{6}\right)$ spectrum of a PLA-PCHO-PLA triblock copolymer; $\mathrm{D}=1.18 \times 10^{-10} \mathrm{~m}^{2} \mathrm{~s}^{-1}$.

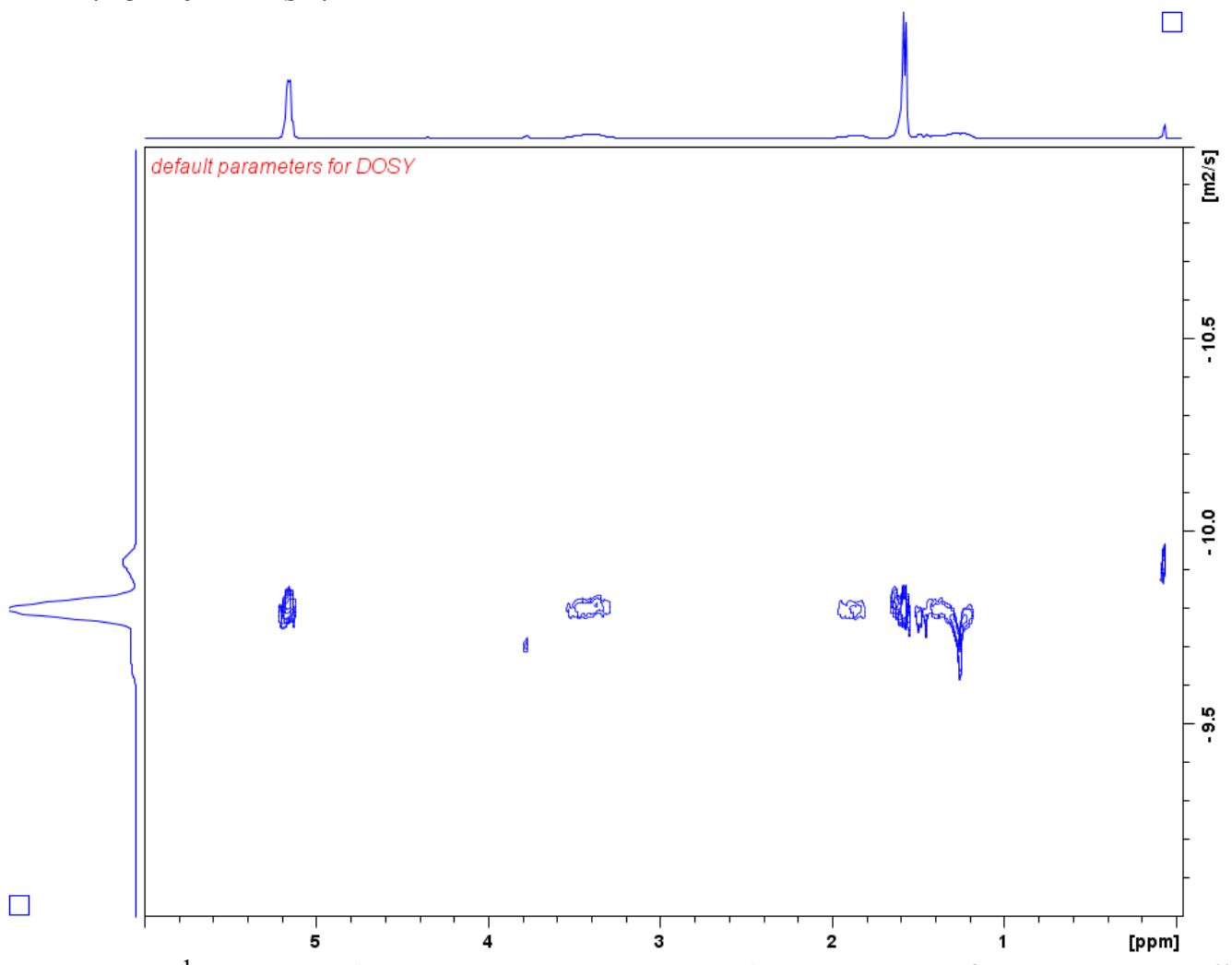

Figure S106. DOSY ${ }^{1} \mathrm{H}$ NMR $\left(500 \mathrm{MHz}, 25{ }^{\circ} \mathrm{C}, \mathrm{C}_{6} \mathrm{D}_{6}\right)$ spectrum of PCHO-PLA diblock copolymer; $\mathrm{D}=1.57 \times 10^{-10} \mathrm{~m}^{2} \mathrm{~s}^{-1}$. 


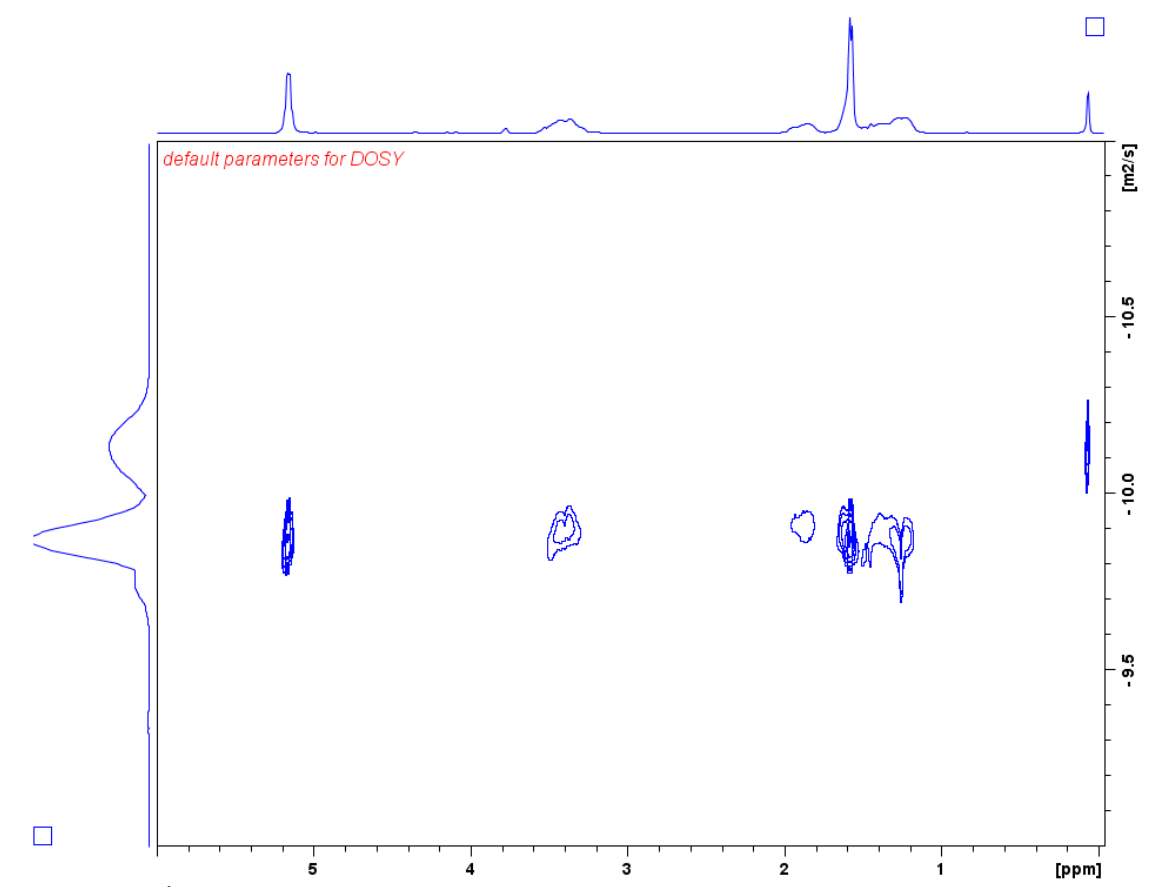

Figure S107. DOSY ${ }^{1} \mathrm{H}$ NMR $\left(500 \mathrm{MHz}, 25{ }^{\circ} \mathrm{C}, \mathrm{C}_{6} \mathrm{D}_{6}\right)$ spectrum of a PCHO-PLA-PCHO triblock copolymer; $\mathrm{D}=1.31 \times 10^{-10} \mathrm{~m}^{2} \mathrm{~s}^{-1}$.

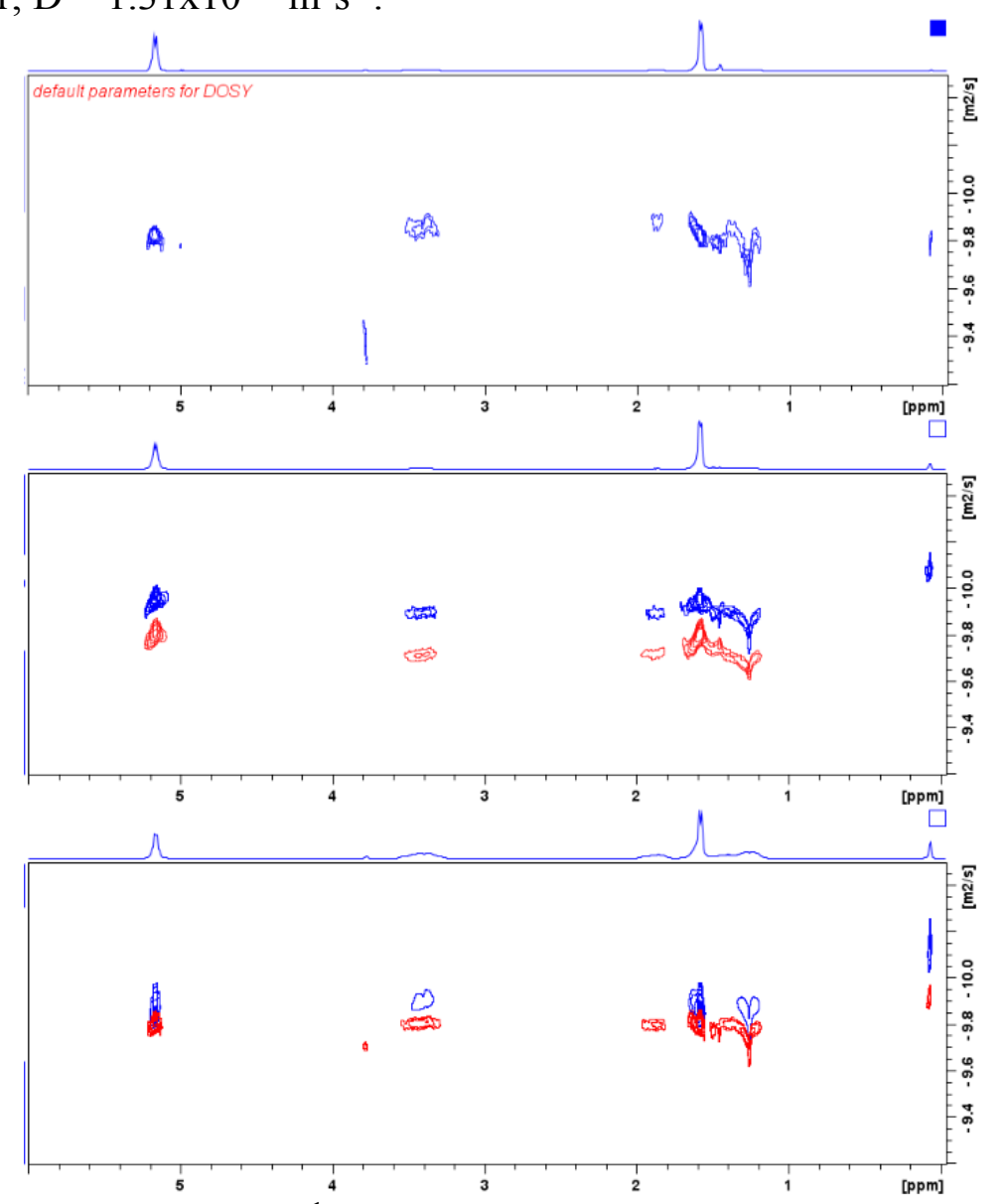

Figure S108. Comparison of DOSY ${ }^{1} \mathrm{H}$ NMR (500 MHz, $\left.25^{\circ} \mathrm{C}, \mathrm{C}_{6} \mathrm{D}_{6}\right)$. PLA, PCHO blend (top), PLA-CHO and PLA-PCHO-PLA (middle), PCHO-PLA and PCHO-PLA-PCHO (bottom). 


\section{Conversion studies}

\section{Conversion of $\mathrm{CHO}$}

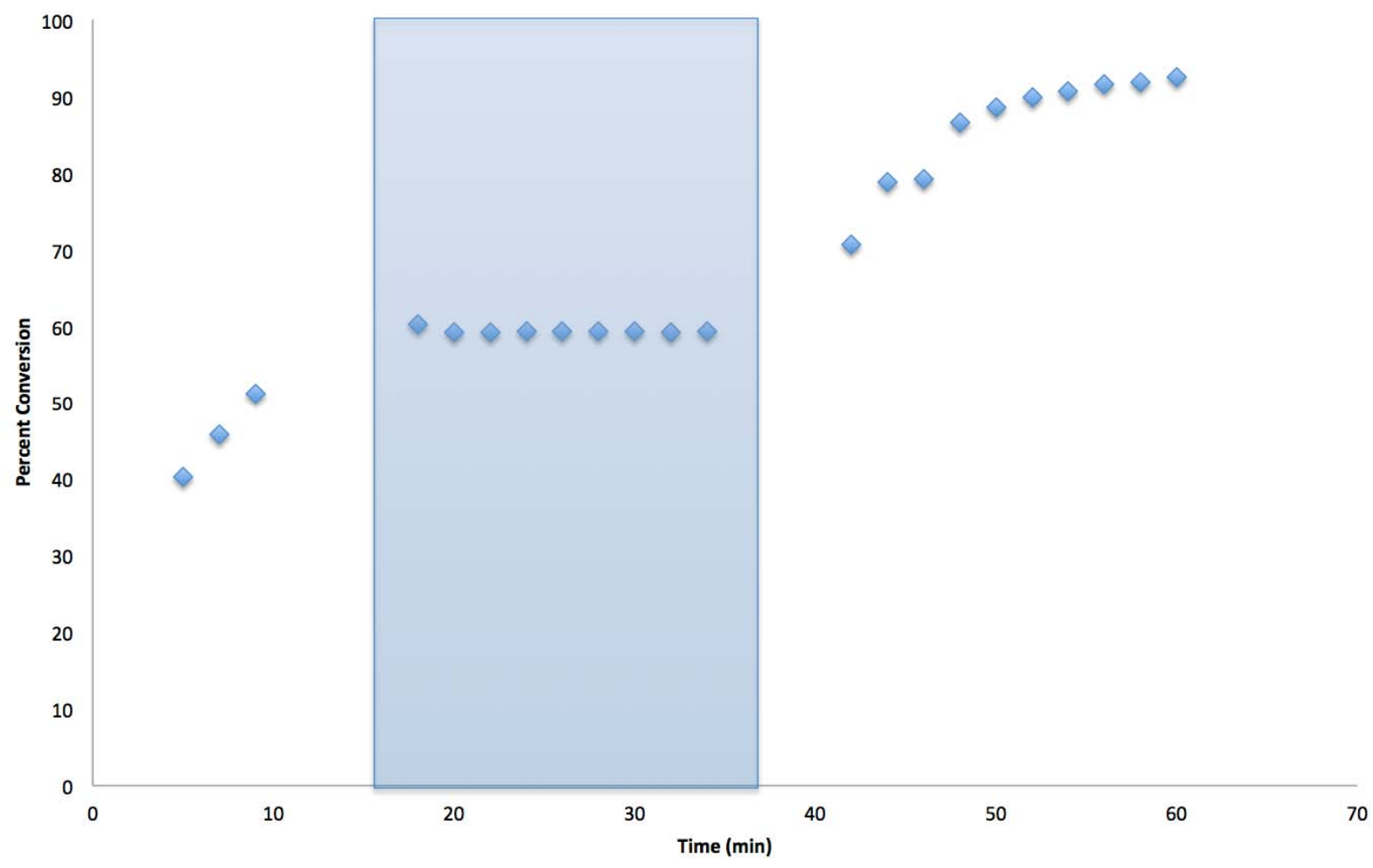

Figure S109. Polymerization of cyclohexene oxide by (salfan) $\mathrm{Zr}(\mathrm{OtBu})_{2}$. Polymerization is switched off by the addition of ${ }^{\mathrm{Ac}} \mathrm{FcBAr}^{\mathrm{F}}$ and switched on by the addition of $\mathrm{CoCp}_{2}$. The corresponding lactide polymerization diagram can be found at DOI: $10.1021 / \mathrm{ja} 505883 \mathrm{u}$. 


\section{Conversion v Mn (LLA)}

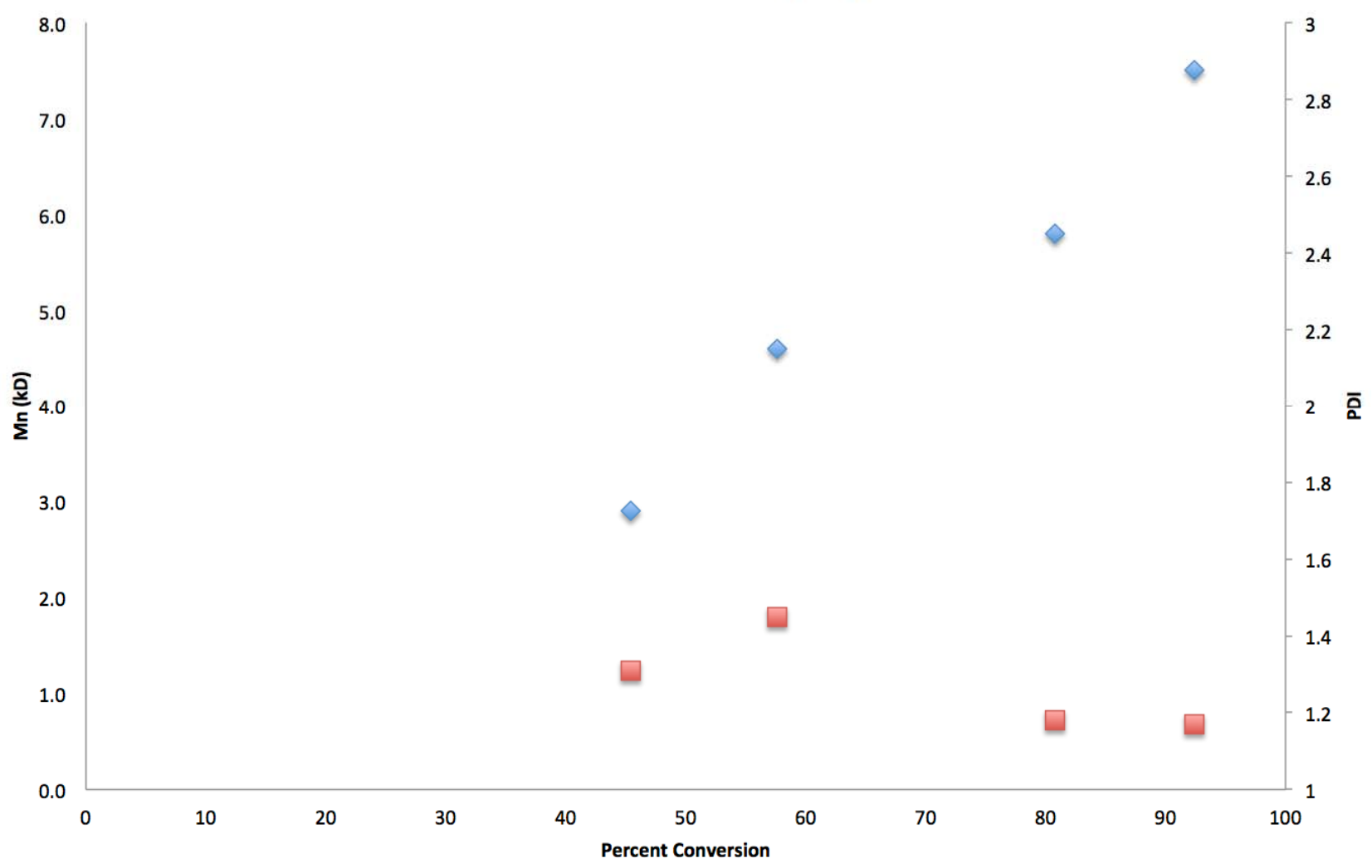

Figure S110. Conversion of L-lactide versus $\mathrm{M}_{\mathrm{n}}$.

\begin{tabular}{|c|c|c|c|c|}
\hline Time & Conversion & $\mathbf{M}_{\mathbf{n}, \mathbf{N M R}}$ & $\mathbf{M}_{\mathbf{n}, \mathbf{G P C}}$ & PDI \\
\hline $\mathbf{6 0}$ & 45.4 & 3.3 & 2.9 & 1.31 \\
\hline $\mathbf{5 0}$ & 57.6 & 4.1 & 4.6 & 1.45 \\
\hline $\mathbf{1 4 0}$ & 80.8 & 5.8 & 5.8 & 1.18 \\
\hline $\mathbf{4 4 0}$ & 92.4 & 6.7 & 7.5 & 1.17 \\
\hline
\end{tabular}




\section{Conversion v $\mathrm{Mn}$ (CHO)}

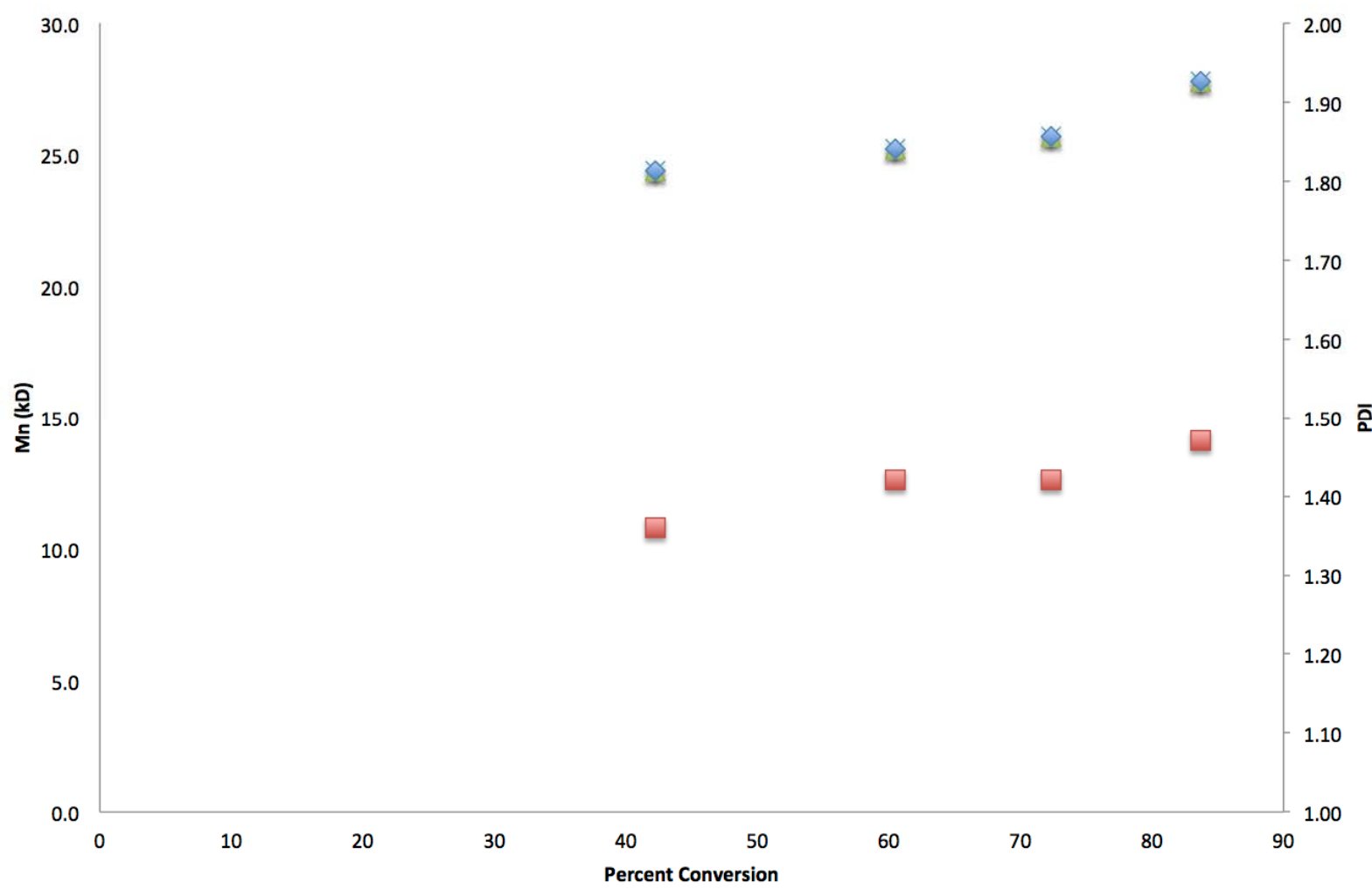

Figure S111. Conversion of cyclohexene oxide versus $\mathrm{M}_{\mathrm{n}}$.

\begin{tabular}{|l|l|l|l|l|}
\hline Time & Conversion & $\mathbf{M}_{\mathbf{n}, \mathbf{N M R}}$ & $\mathbf{M}_{\mathbf{n}, \mathbf{G P C}}$ & $\mathbf{P D I}$ \\
\hline $\mathbf{1 3}$ & 42.2 & 4.1 & 24.4 & 1.36 \\
\hline $\mathbf{2 8}$ & 60.5 & 5.9 & 25.2 & 1.42 \\
\hline $\mathbf{4 2}$ & 72.3 & 7.1 & 25.7 & 1.42 \\
\hline $\mathbf{7 1}$ & 83.7 & 8.2 & 27.8 & 1.47 \\
\hline
\end{tabular}




\section{Conversion vs. Time (LA)}

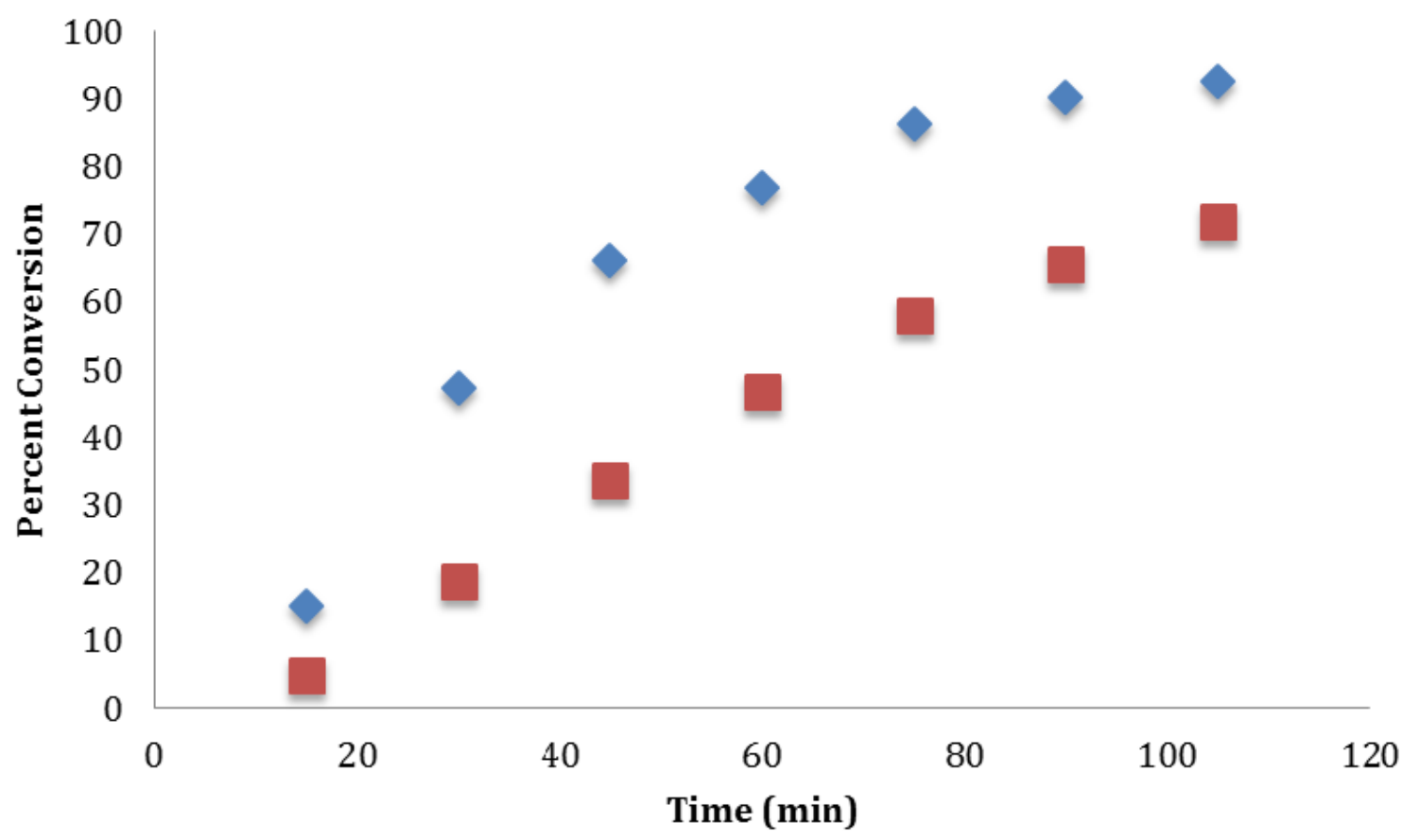

Figure S112. Conversion of 100 equivalents of L-lactide to PLA vs time with (blue) and without (orange) 100 equivalents of cyclohexene oxide $(0.66 \mathrm{M})$ in $4: 1 \mathrm{C}_{6} \mathrm{D}_{6}: o-\mathrm{F}_{2} \mathrm{C}_{6} \mathrm{H}_{4}$.

\section{Conversion vs. Time (CHO)}

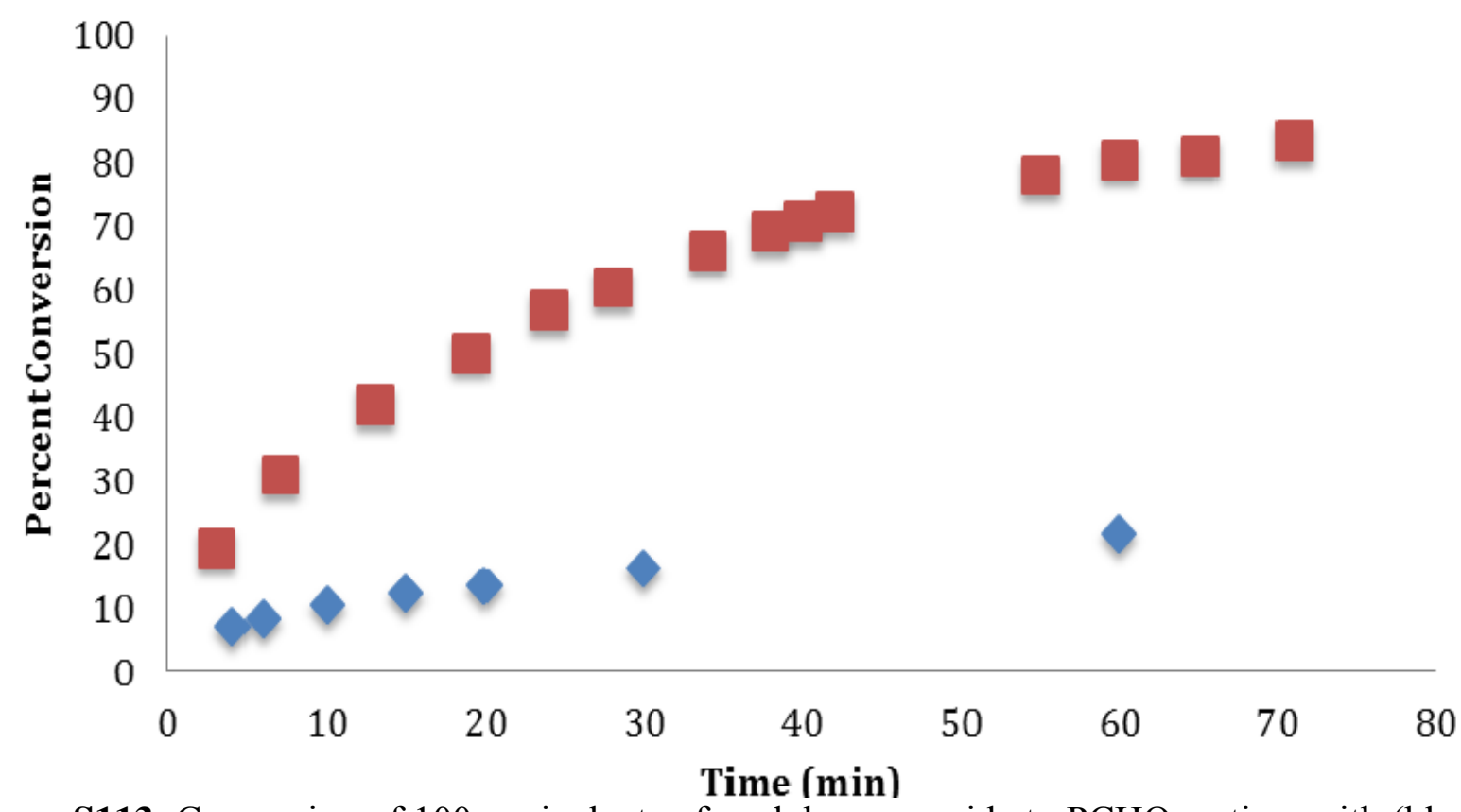

Figure S113. Conversion of 100 equivalents of cyclohexene oxide to $\mathrm{PCHO}$ vs time with (blue) and without (orange) 100 equivalents of L-lactide $(0.66 \mathrm{M})$ in $4: 1 \mathrm{C}_{6} \mathrm{D}_{6}: o-\mathrm{F}_{2} \mathrm{C}_{6} \mathrm{H}_{4}$. 


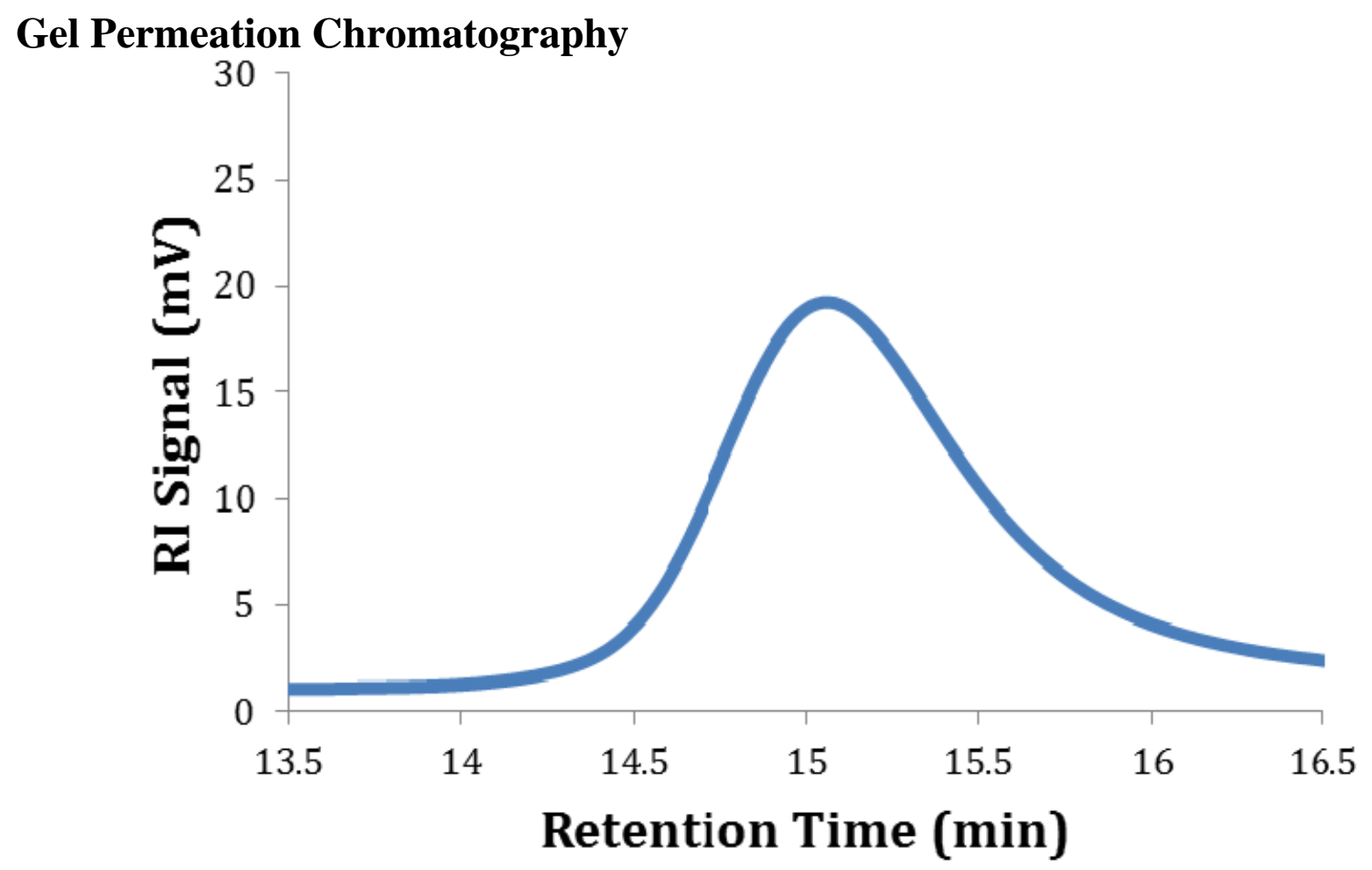

Figure S114. Table 2, entry 1: Polymerization of 100 equivalents of $\beta$-butyrolactone in the presence of 100 equivalents of cyclohexene oxide with catalyst in the reduced state; $M_{n}=9538$ $\mathrm{Da}, \mathrm{M}_{\mathrm{w}}=10802 \mathrm{Da}, \mathrm{PDI}=1.13$.

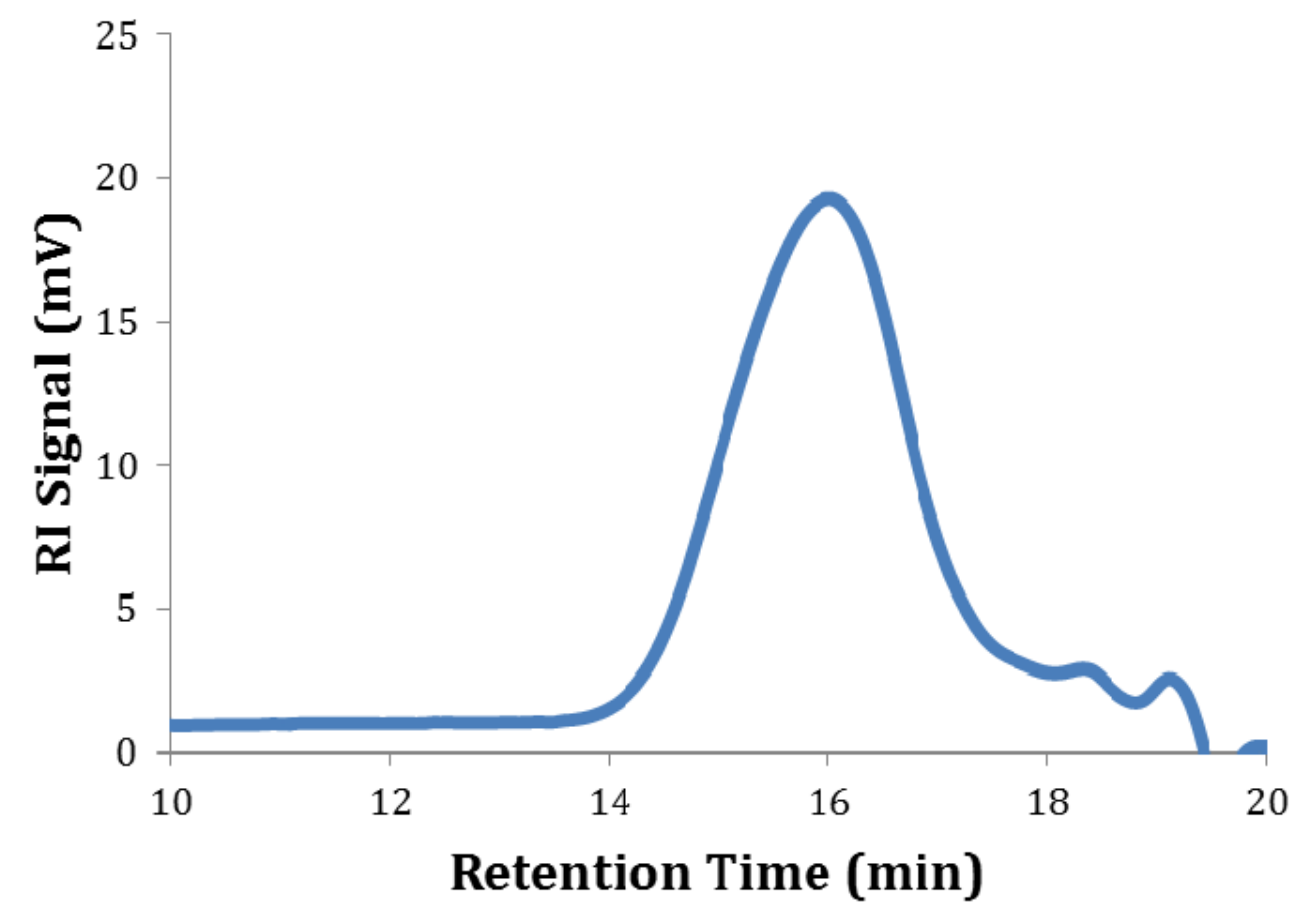

Figure S115. Table 2, entry 2: Polymerization of 100 equivalents of cyclohexene oxide in the presence of 100 equivalents of $\beta$-butyrolactone with catalyst in the reduced state and subsequently oxidized; $\mathrm{M}_{\mathrm{n}}=3884 \mathrm{Da}, \mathrm{M}_{\mathrm{w}}=5983 \mathrm{Da}, \mathrm{PDI}=1.54$. 


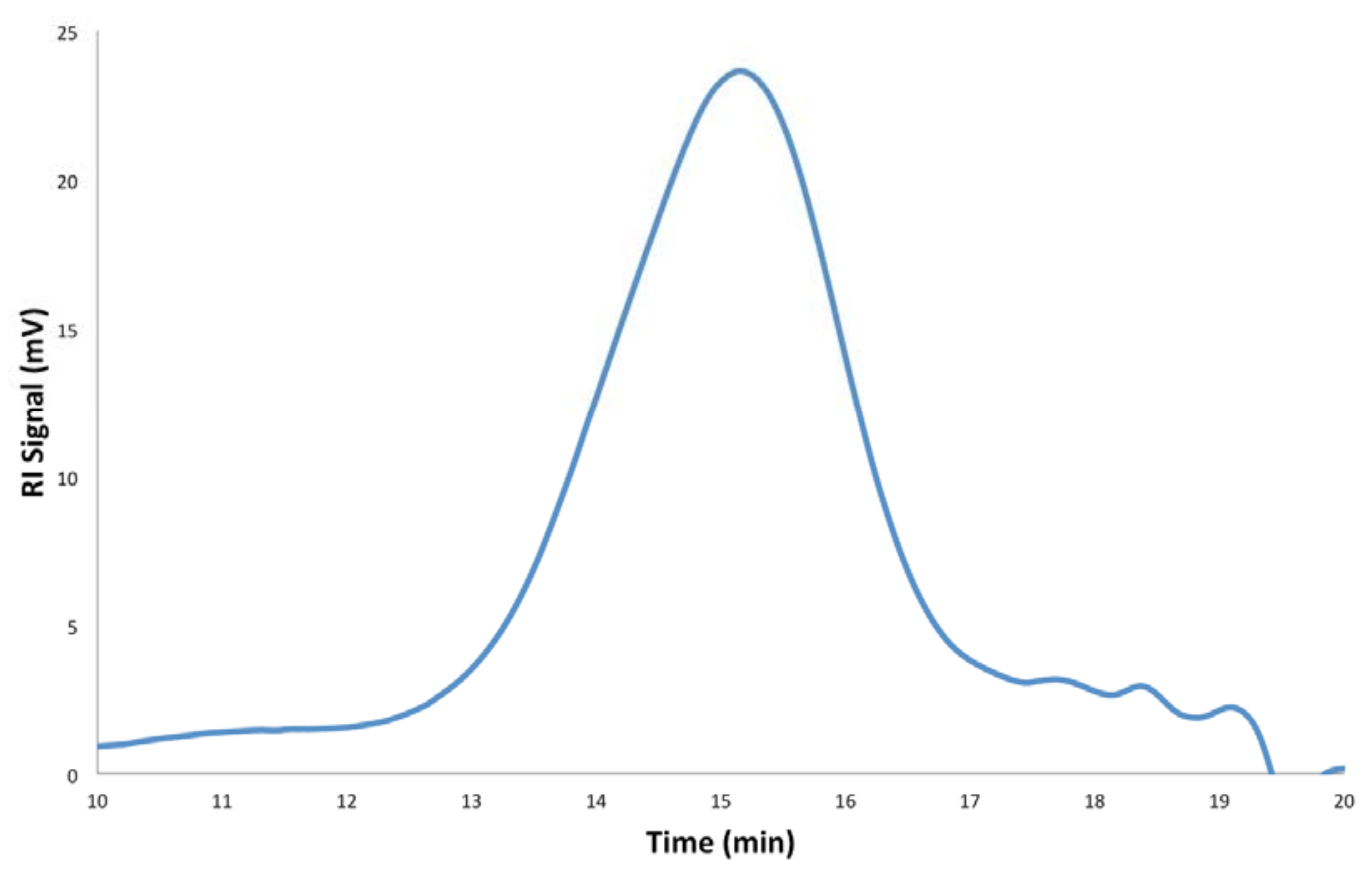

Figure S116. Table 2, entry 3: Polymerization of 100 equivalents of $\beta$-butyrolactone in the presence of 100 equivalents of cyclohexene oxide with catalyst in the oxidized state; $M_{n}=8347$ $\mathrm{Da}, \mathrm{M}_{\mathrm{w}}=12604 \mathrm{Da}, \mathrm{PDI}=1.51$.

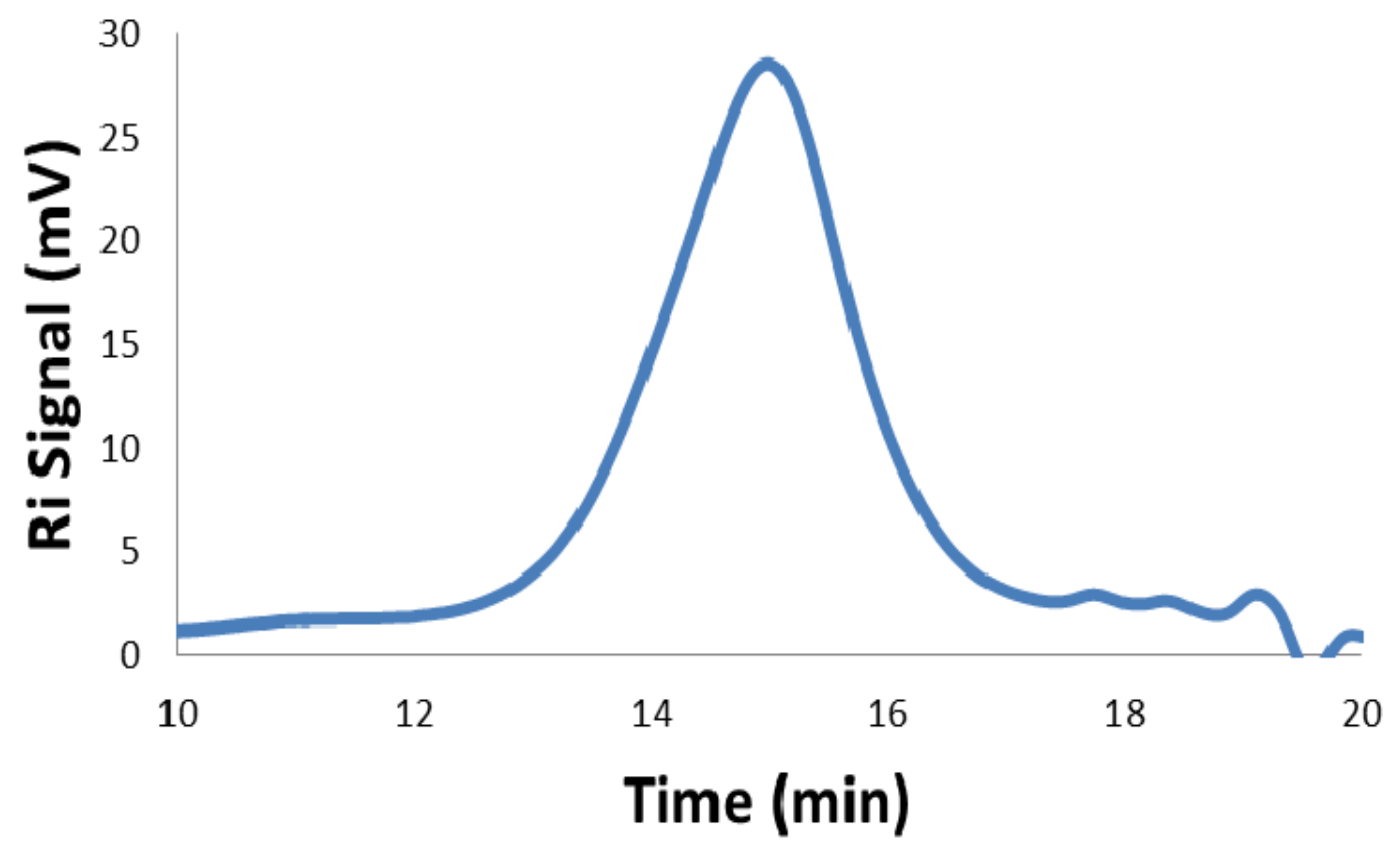

Figure S117. Table 2, entry 4: Polymerization of 100 equivalents of $\beta$-butyrolactone in the presence of 100 equivalents of cyclohexene oxide with catalyst in the oxidized state and subsequently reduced; $\mathrm{M}_{\mathrm{n}}=9680 \mathrm{Da}, \mathrm{M}_{\mathrm{w}}=15004 \mathrm{Da}, \mathrm{PDI}=1.55$. 


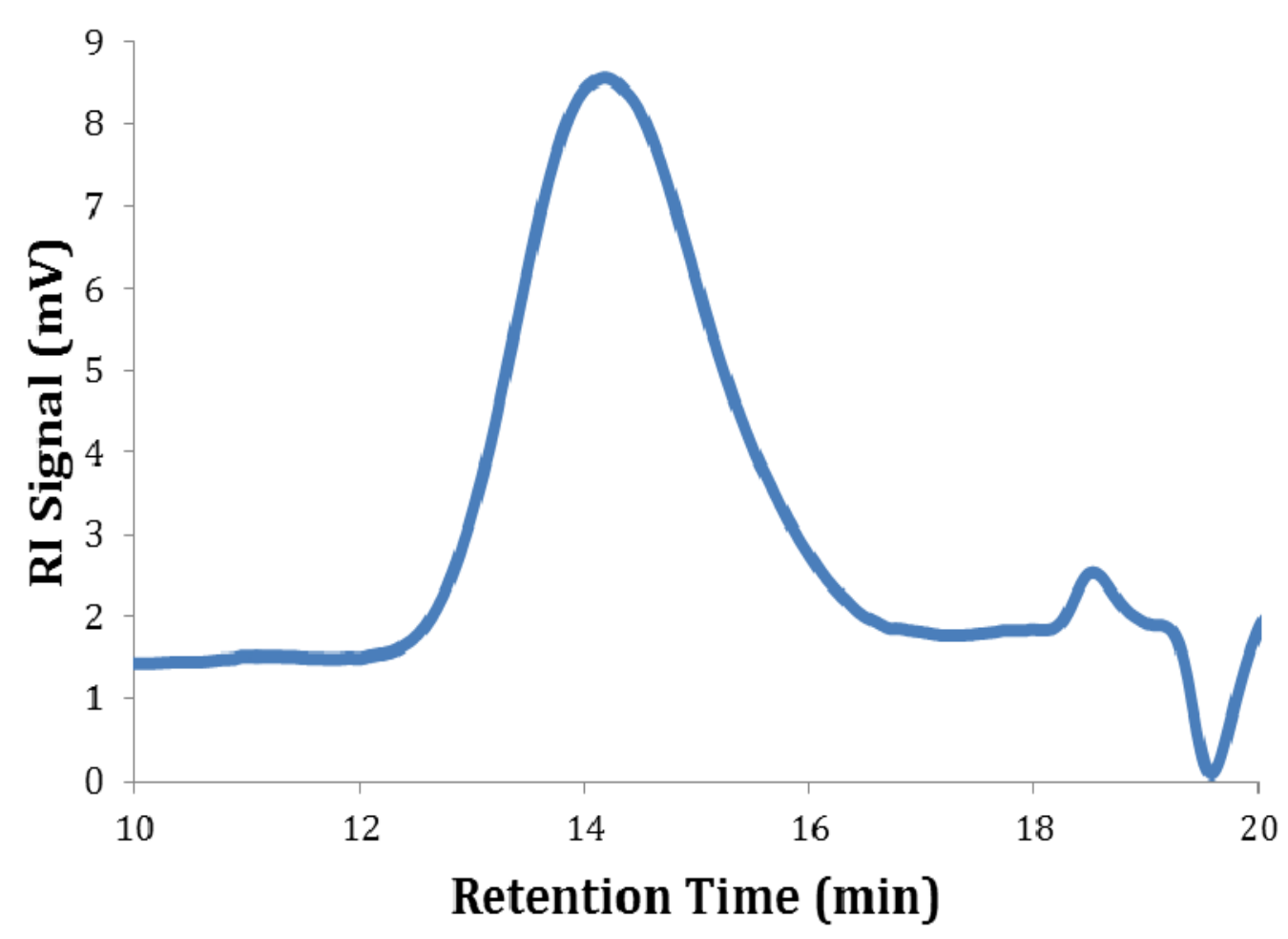

Figure S118. Table 2, entry 6: Polymerization of 100 equivalents of propylene oxide in the presence of 100 equivalents of L-lactide with catalyst in the oxidized state and subsequently reduced; $\mathrm{M}_{\mathrm{n}}=16422 \mathrm{Da}, \mathrm{M}_{\mathrm{w}}=27033 \mathrm{Da}, \mathrm{PDI}=1.65$.

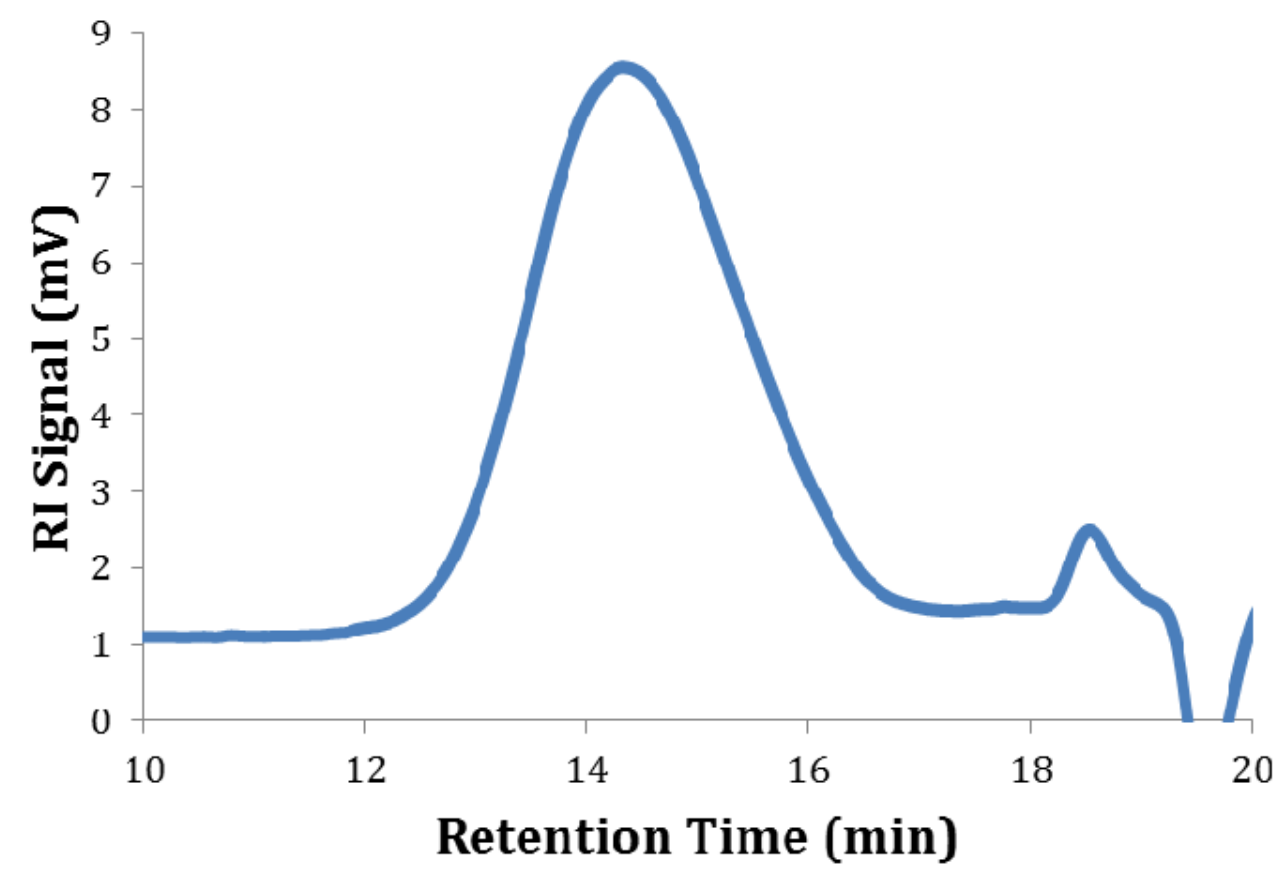

Figure S119. Table 2, entry 8: Polymerization of 100 equivalents of L-lactide in the presence of 100 equivalents of oxetane with catalyst in the oxidized state and subsequently reduced; $\mathrm{M}_{\mathrm{n}}=$ $14091 \mathrm{Da}, \mathrm{M}_{\mathrm{w}}=23348 \mathrm{Da}, \mathrm{PDI}=1.66$. 


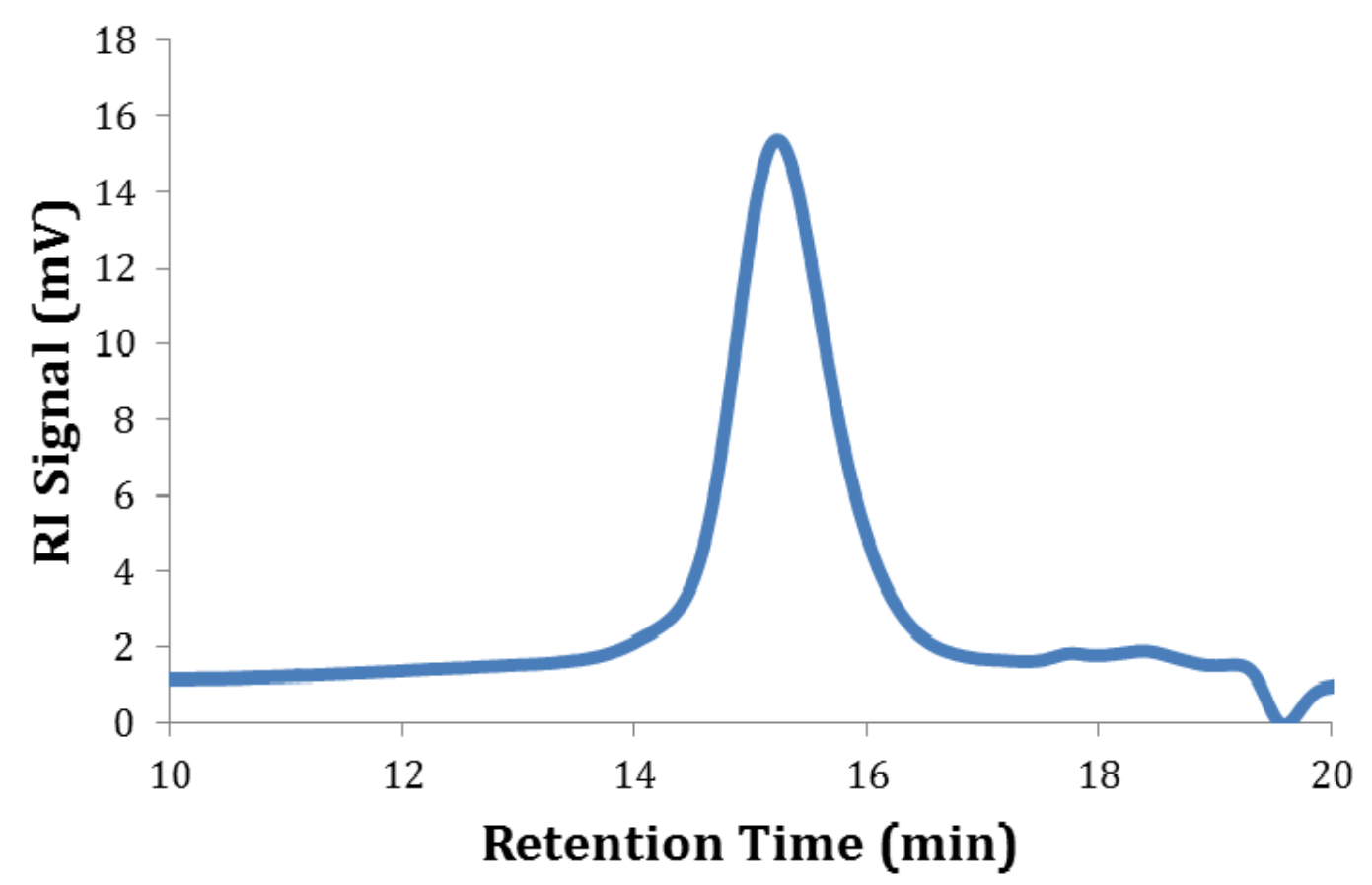

Figure S120. Table 2, entry 9: Polymerization of 100 equivalents of L-lactide in the presence of 100 equivalents of cyclohexene oxide with catalyst in the reduced state; $\mathrm{M}_{\mathrm{n}}=7936 \mathrm{Da}, \mathrm{M}_{\mathrm{w}}=$ $9155 \mathrm{Da}, \mathrm{PDI}=1.15$.

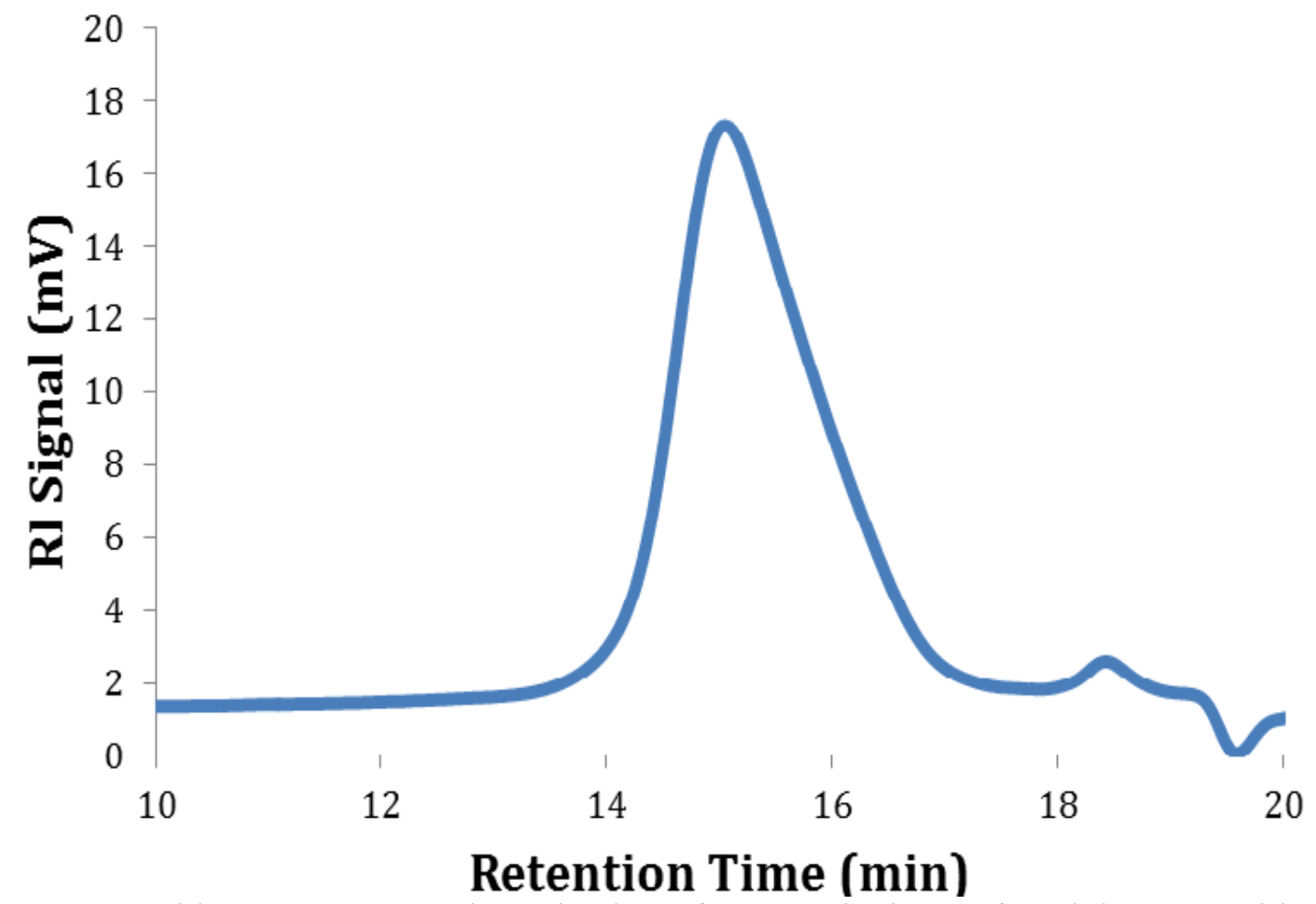

Figure S121. Table 2, entry 10: Polymerization of 100 equivalents of cyclohexene oxide in the presence of 100 equivalents of L-lactide with catalyst in the reduced state and subsequently oxidized; $\mathrm{M}_{\mathrm{n}}=7640 \mathrm{Da}, \mathrm{M}_{\mathrm{w}}=9921 \mathrm{Da}, \mathrm{PDI}=1.30$. 


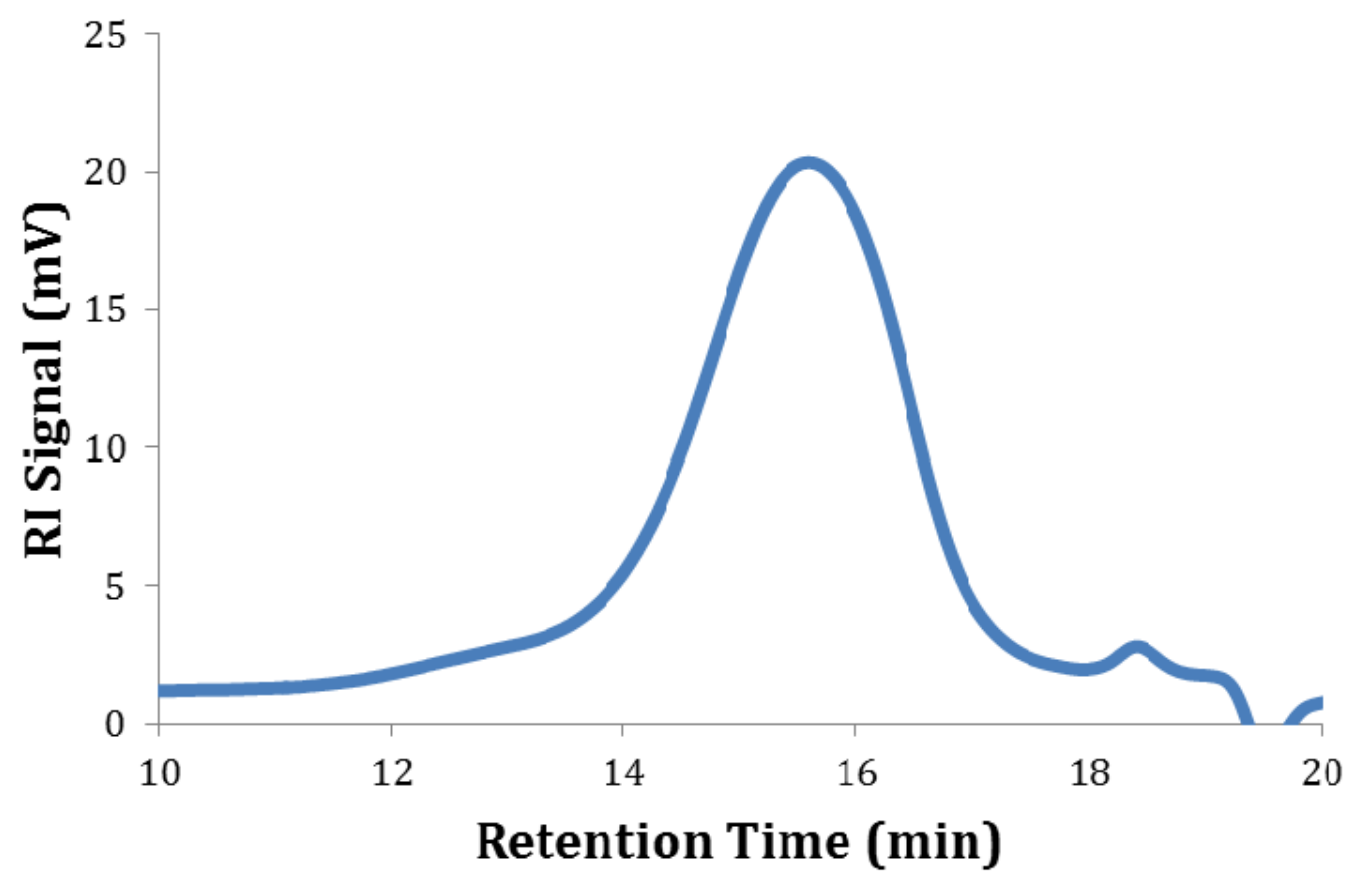

Figure S122. Table 2, entry 11: Polymerization of 100 equivalents of cyclohexene oxide in the presence of 100 equivalents of L-lactide with catalyst in the oxidized state; $\mathrm{M}_{\mathrm{n}}=5481 \mathrm{Da}, \mathrm{M}_{\mathrm{w}}=$ $8455 \mathrm{Da}, \mathrm{PDI}=1.54$.

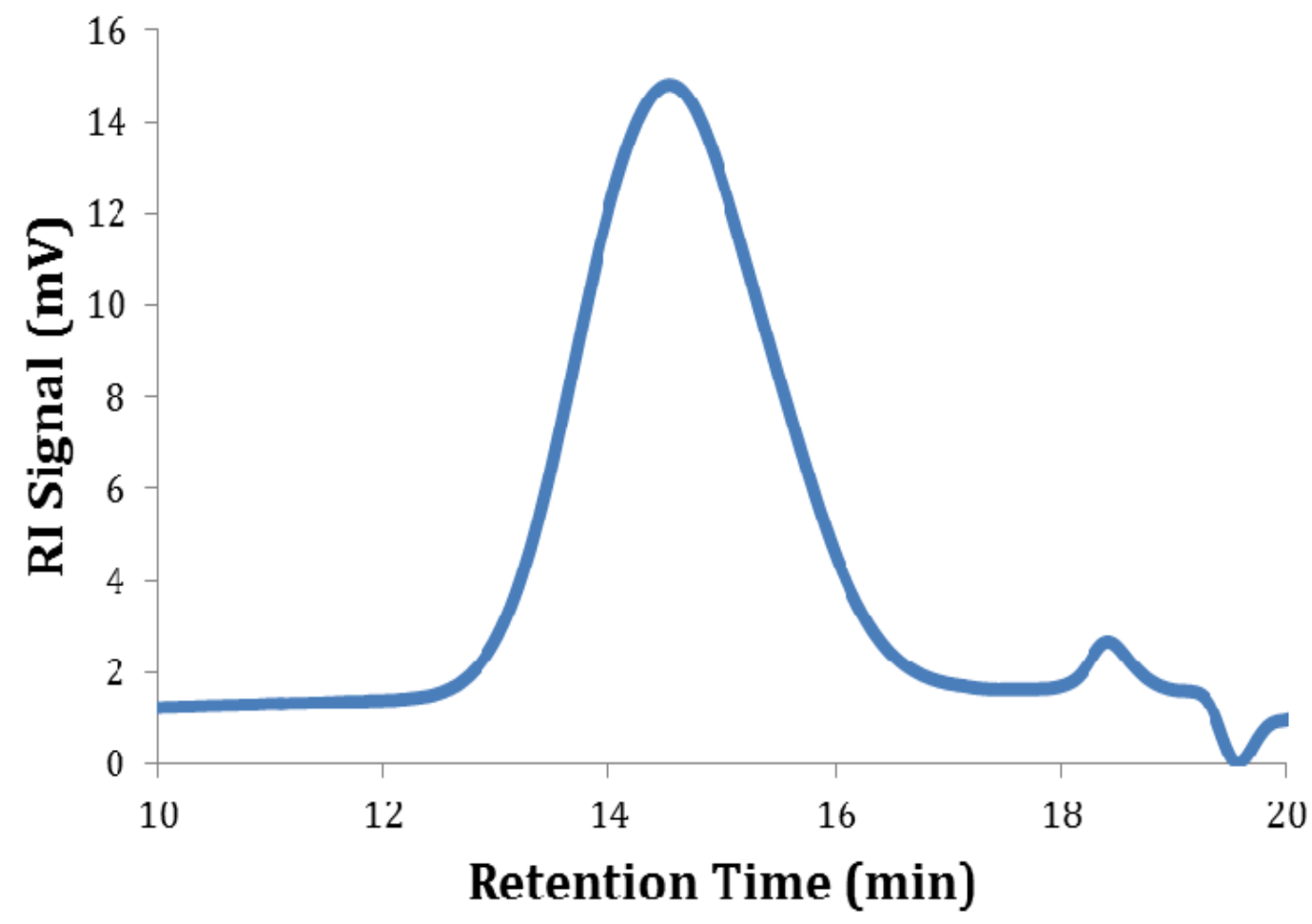

Figure S123. Table 2, entry 12: Polymerization of 100 equivalents of L-lactide in the presence of 100 equivalents of cyclohexene oxide with catalyst in the oxidized state and subsequently reduced; $\mathrm{M}_{\mathrm{n}}=12339 \mathrm{Da}, \mathrm{M}_{\mathrm{w}}=17806 \mathrm{Da}, \mathrm{PDI}=1.44$. 


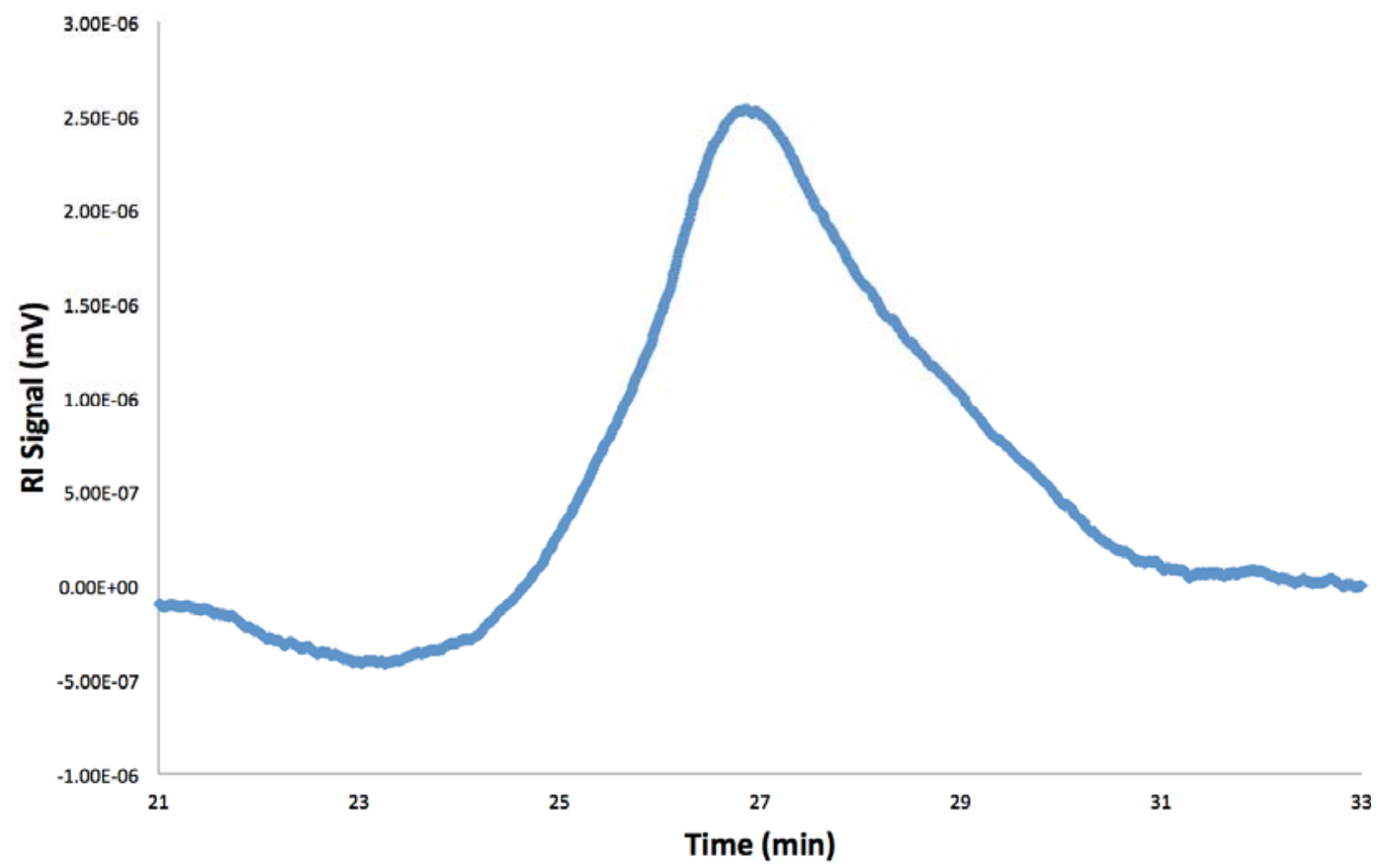

Figure S124. Table 4, entry 1. Table S1, entry 2. Table S2, entry 1: Polymerization of 100 equivalents of L-lactide and cyclohexene oxide, monomers added sequentially, using catalyst redox switch "red-ox"; $\mathrm{M}_{\mathrm{n}}=11400 \mathrm{Da}, \mathrm{M}_{\mathrm{w}}=15048 \mathrm{Da}, \mathrm{PDI}=1.32$.

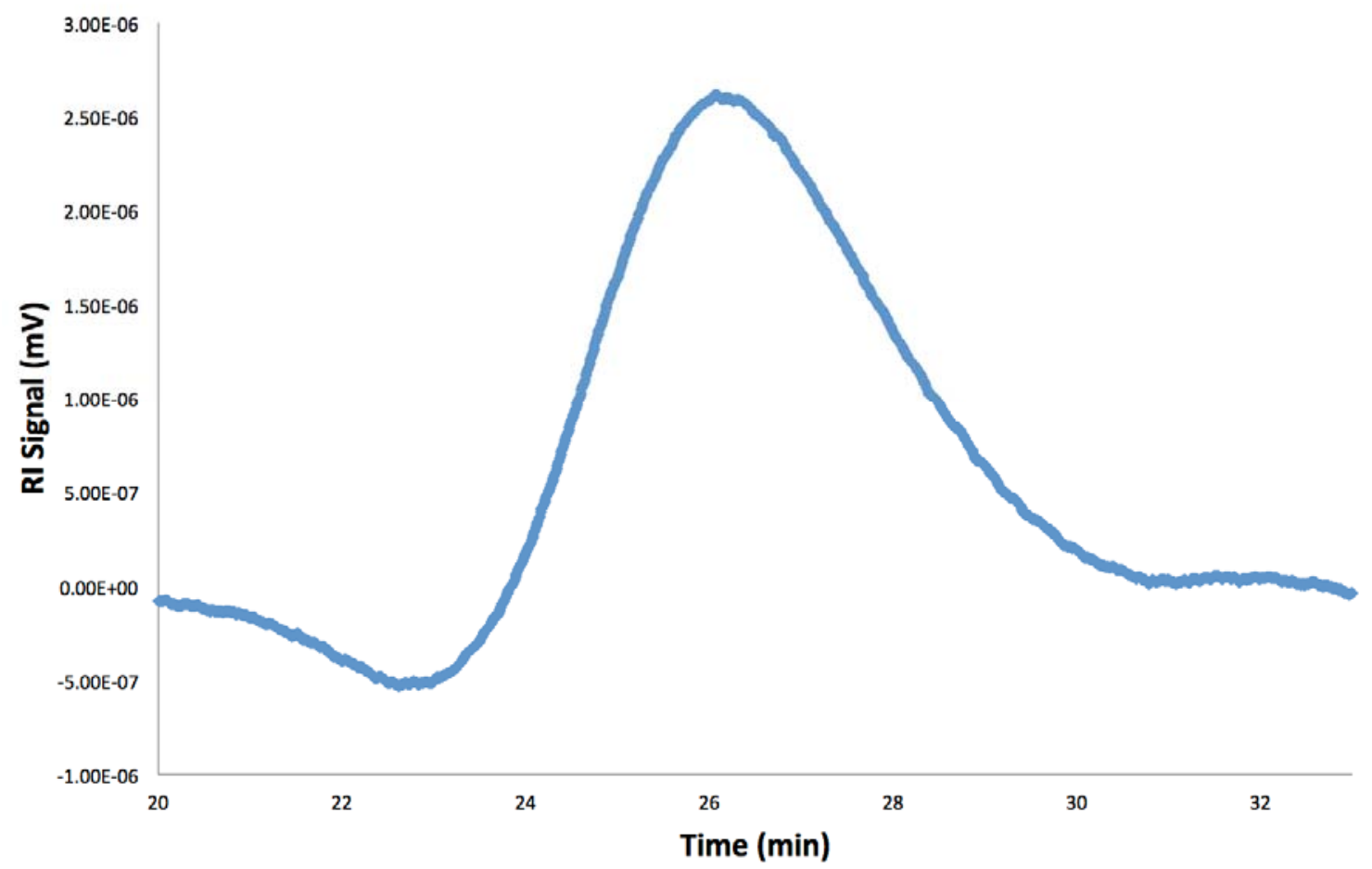

Figure S125. Table 4, entry 2. Table S1, entry 3. Table S3, entry 1: Polymerization of 100 equivalents of L-Lactide, cyclohexene oxide, and L-lactide, monomers added sequentially, using catalyst redox switch "red-ox-red"; $\mathrm{M}_{\mathrm{n}}=16940 \mathrm{Da}, \mathrm{M}_{\mathrm{w}}=21175 \mathrm{Da}, \mathrm{PDI}=1.25$. 


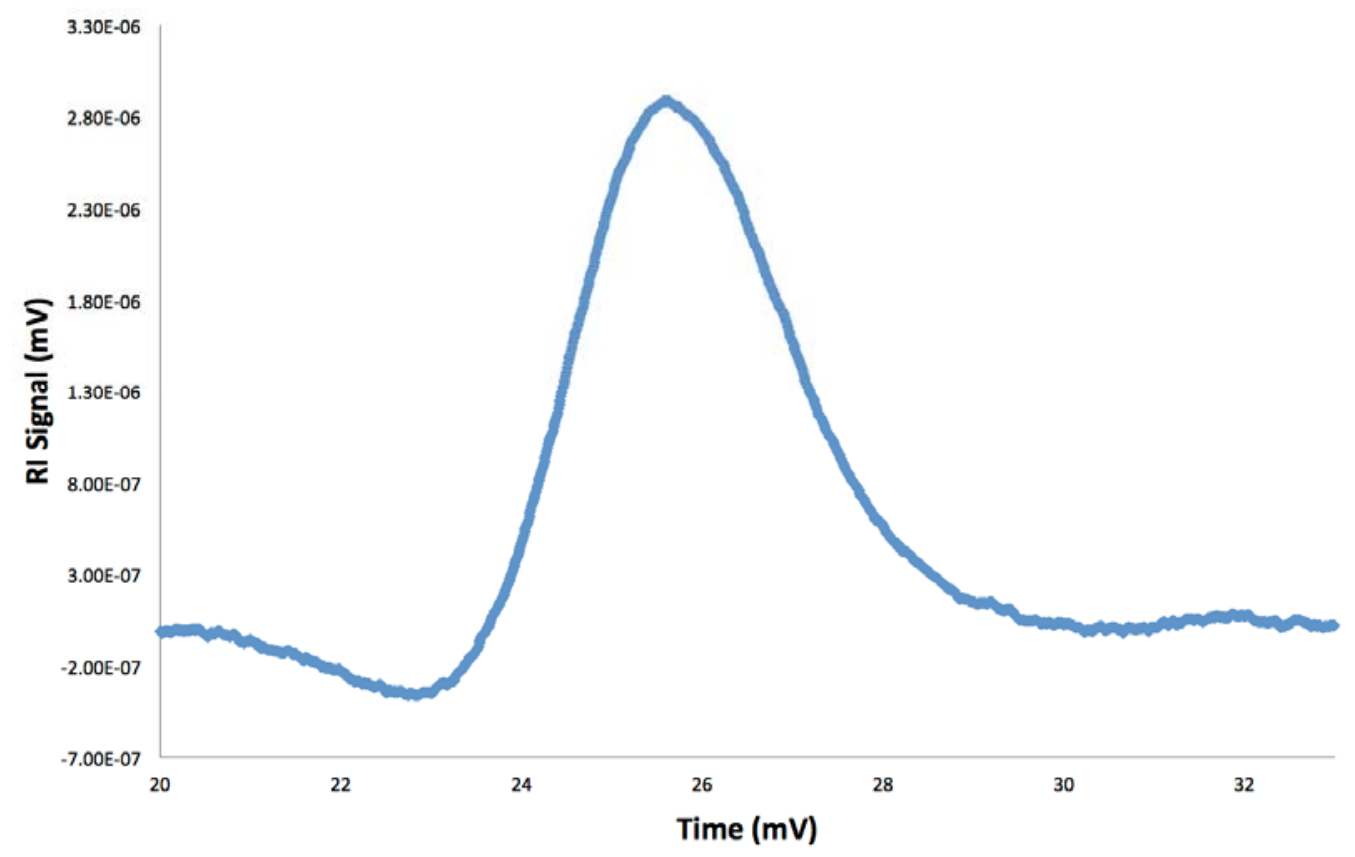

Figure S126. Table 4, entry 3. Table S1, entry 5. Table S4, entry 1: Polymerization of 100 equivalents of L-lactide, cyclohexene oxide, monomers added sequentially, using catalyst redox switch "ox-red"; $\mathrm{M}_{\mathrm{n}}=13860 \mathrm{Da}, \mathrm{M}_{\mathrm{w}}=23007 \mathrm{Da}, \mathrm{PDI}=1.66$.

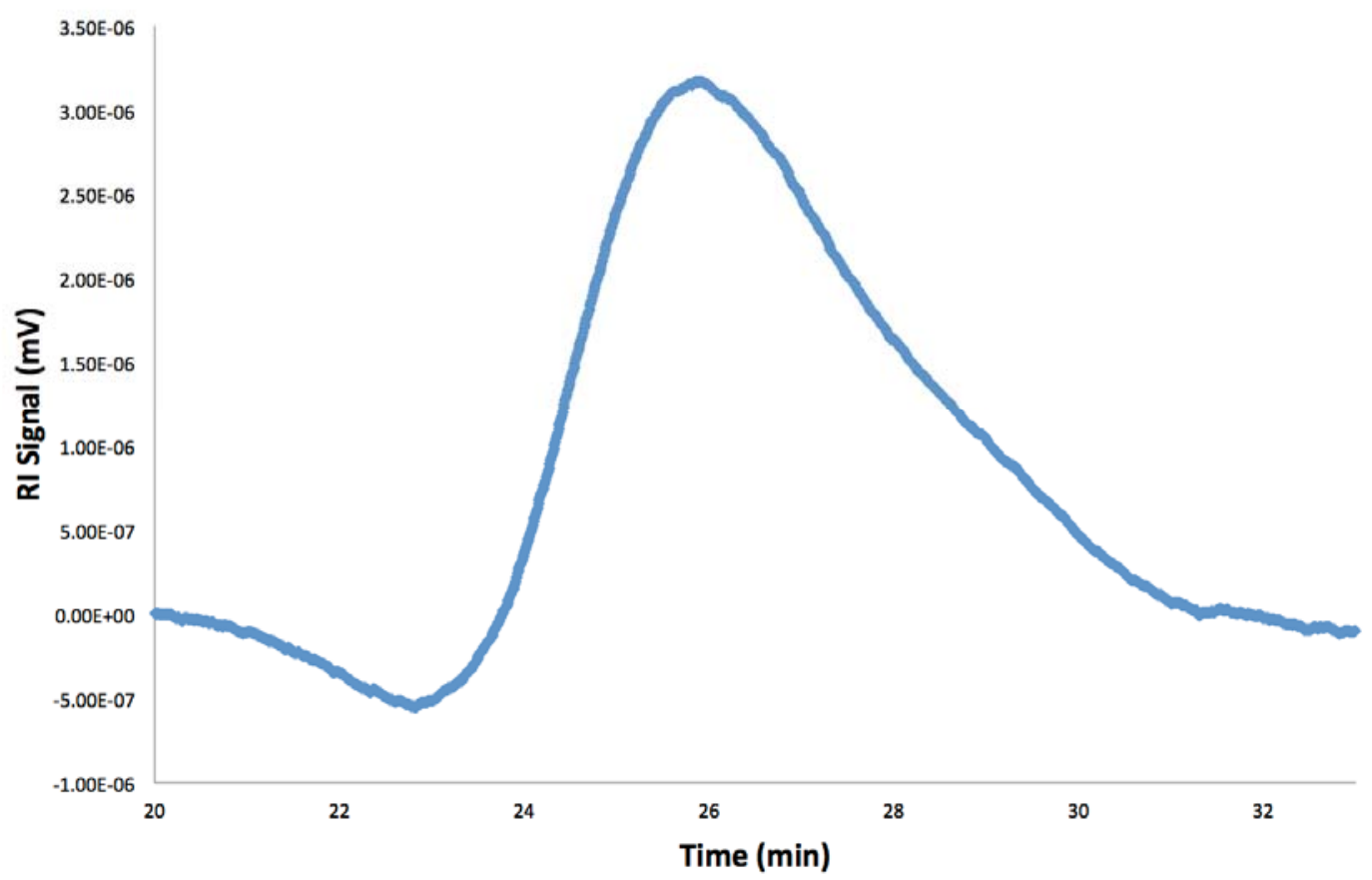

Figure S127. Table 4, entry 4. Table S1, entry 6. Table S5, entry 1: Polymerization of 100 equivalents of cyclohexene oxide, L-lactide, and cyclohexene oxide, monomers added sequentially, using catalyst redox switch "ox-red-ox"; $\mathrm{M}_{\mathrm{n}}=13340 \mathrm{Da}, \mathrm{M}_{\mathrm{w}}=20410 \mathrm{Da}, \mathrm{PDI}=$ 1.53 . 


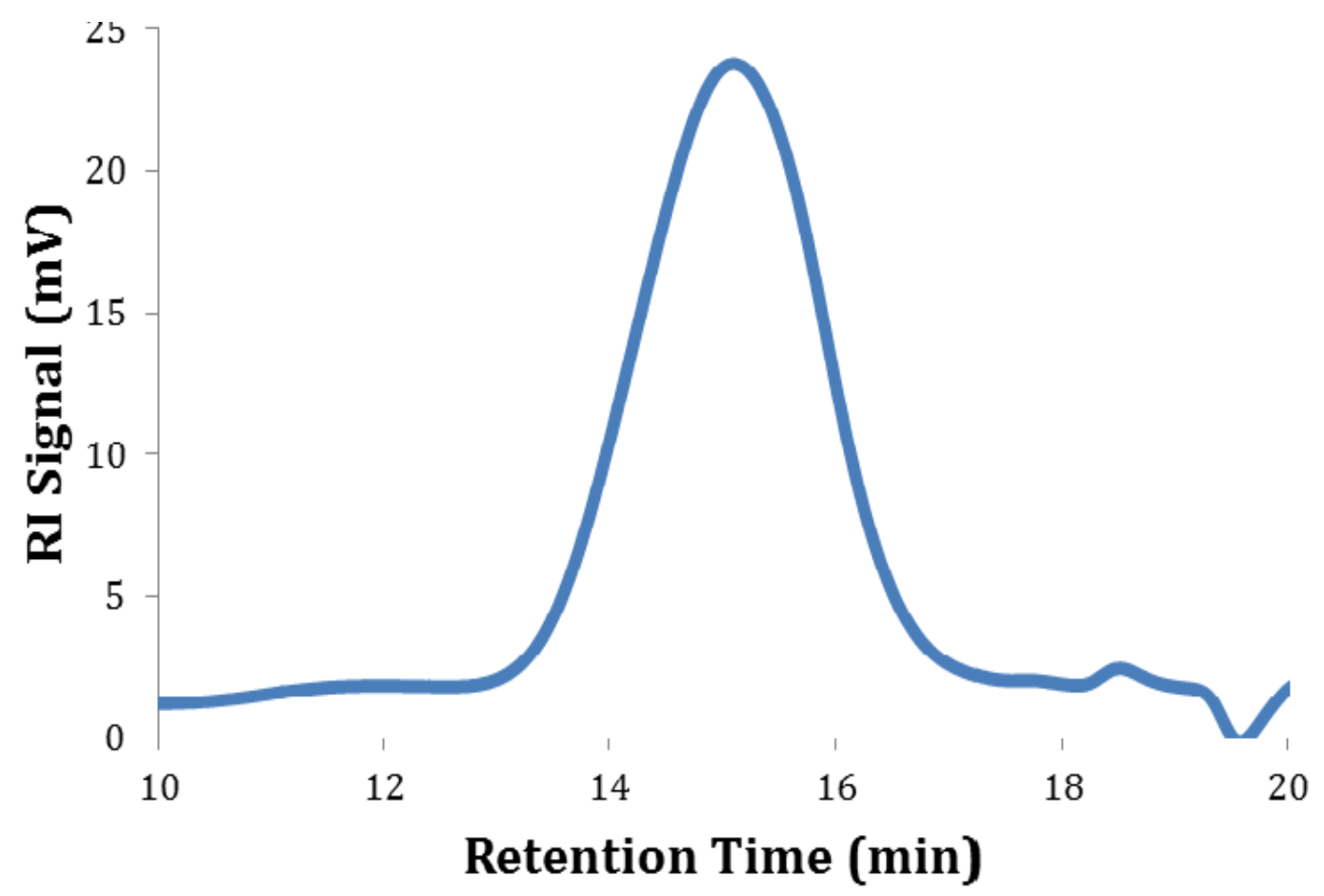

Figure S128. Table 4, entry 5: Polymerization of 100 equivalents of cyclohexene oxide, Llactide, and propylene oxide, monomers added sequentially, using catalyst redox switch "ox-redox"; $\mathrm{M}_{\mathrm{n}}=8996 \mathrm{Da}, \mathrm{M}_{\mathrm{w}}=13841 \mathrm{Da}, \mathrm{PDI}=1.54$.

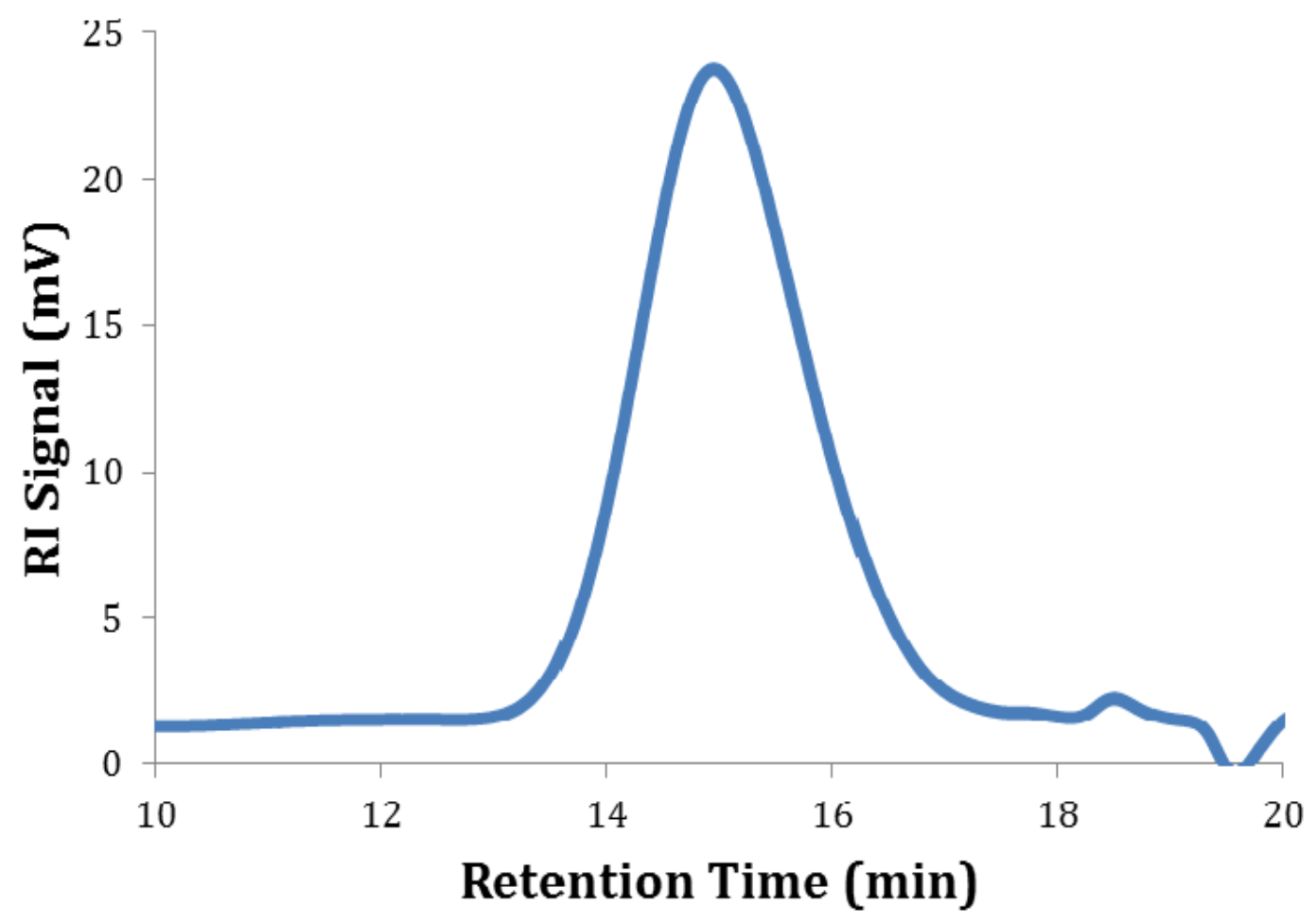

Figure S129. Table 4, entry 6: Polymerization of 100 equivalents of L-lactide, cyclohexene oxide, and $\beta$-butyrolactone, monomers added sequentially, using catalyst redox switch "red-oxred"; $\mathrm{M}_{\mathrm{n}}=8945 \mathrm{Da}, \mathrm{M}_{\mathrm{w}}=13541 \mathrm{Da}, \mathrm{PDI}=1.51$. 


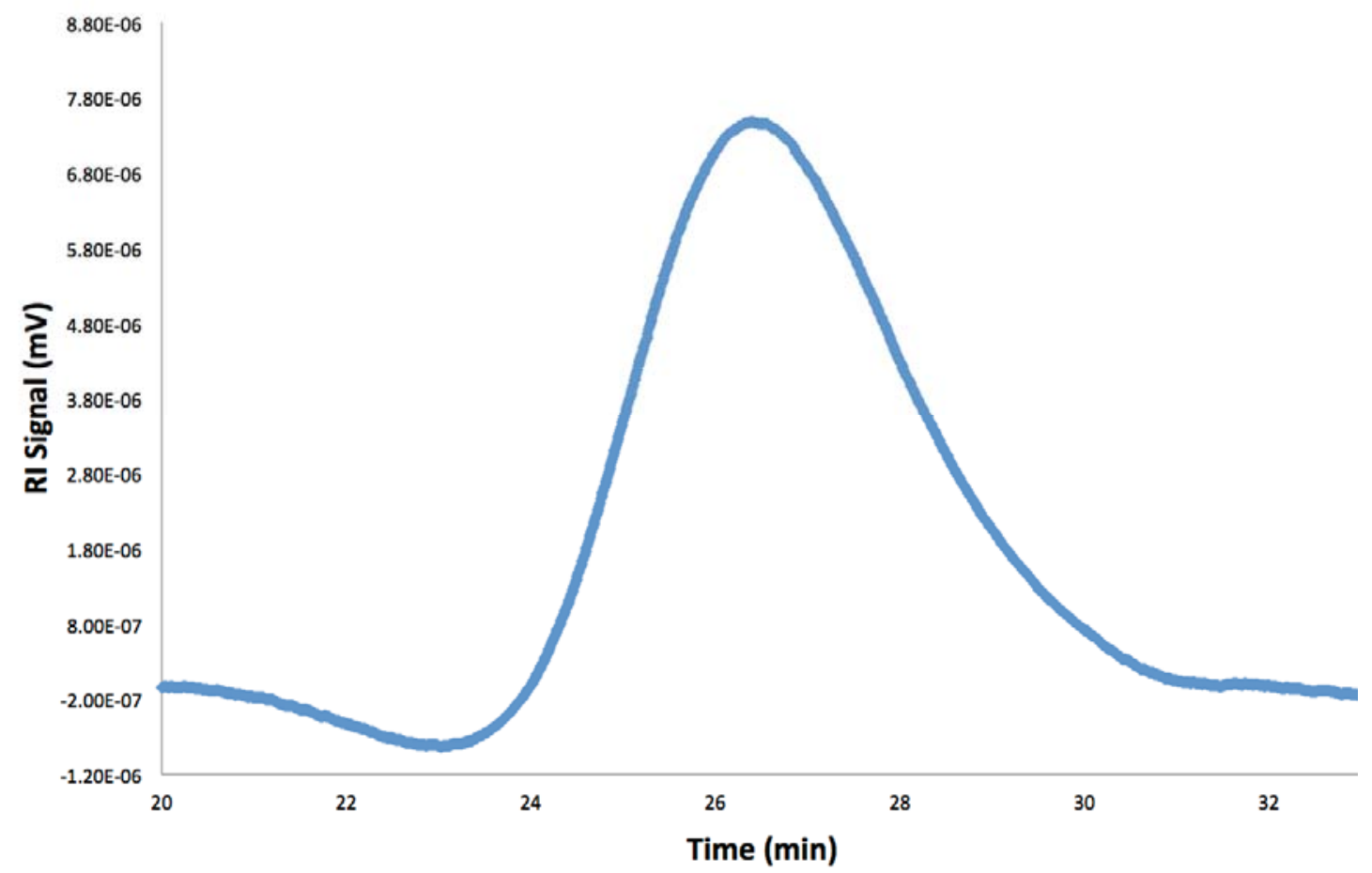

Figure S130. Polymerization of 100 equivalents of cyclohexene oxide by $\left[\mathrm{H}_{2}\right.$ (salfan)][BAr $\left.{ }^{\mathrm{F}}\right] ; \mathrm{M}_{\mathrm{n}}$ $=12870 \mathrm{Da}, \mathrm{M}_{\mathrm{w}}=17889 \mathrm{Da}, \mathrm{PDI}=1.39$.

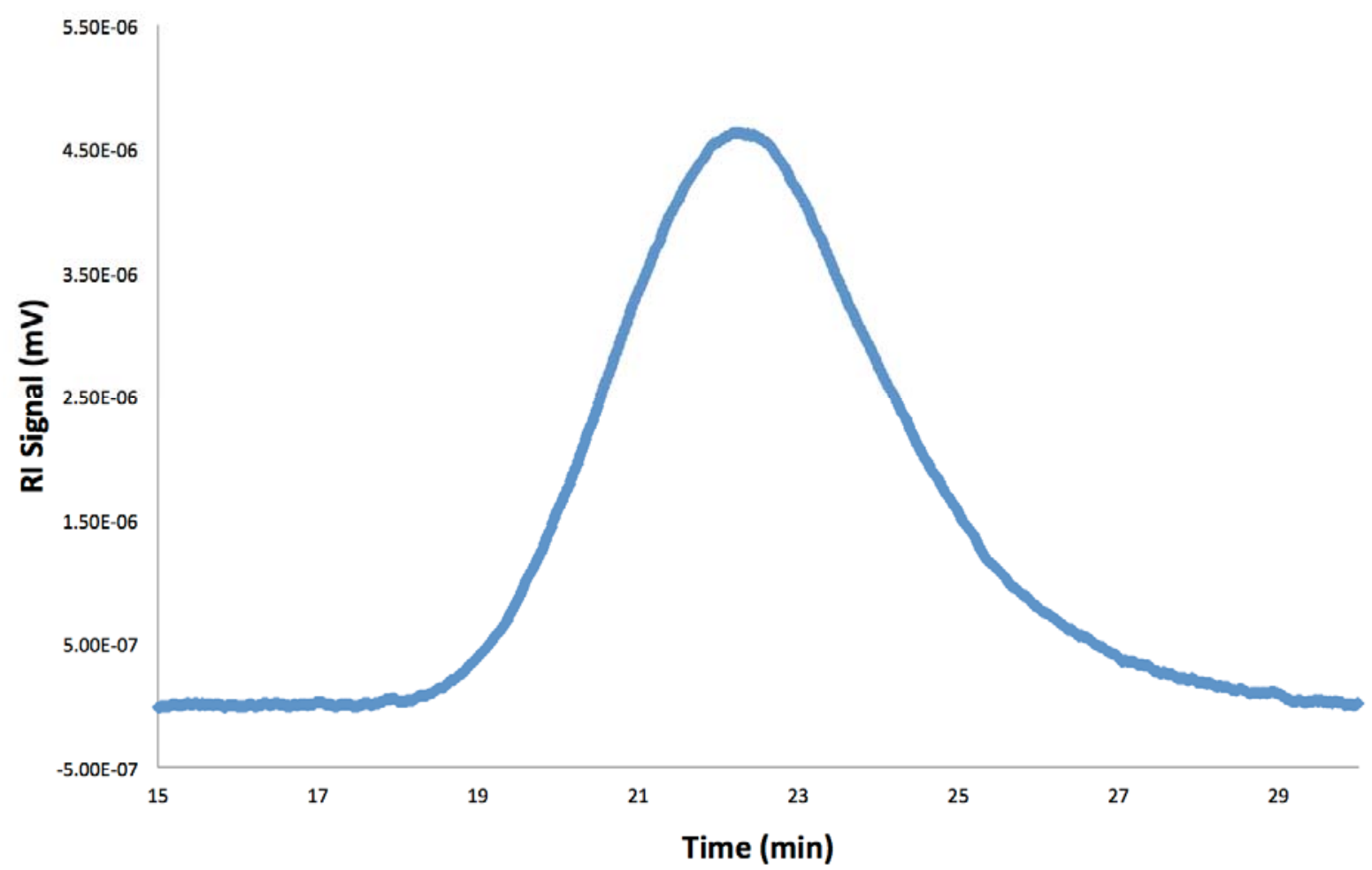

Figure S131. Polymerization of 100 equivalents of cyclohexene oxide by ${ }^{A c} F c B A r r^{F} ; M_{n}=$ $111400 \mathrm{Da}, \mathrm{M}_{\mathrm{w}}=158188 \mathrm{Da}, \mathrm{PDI}=1.42$. 


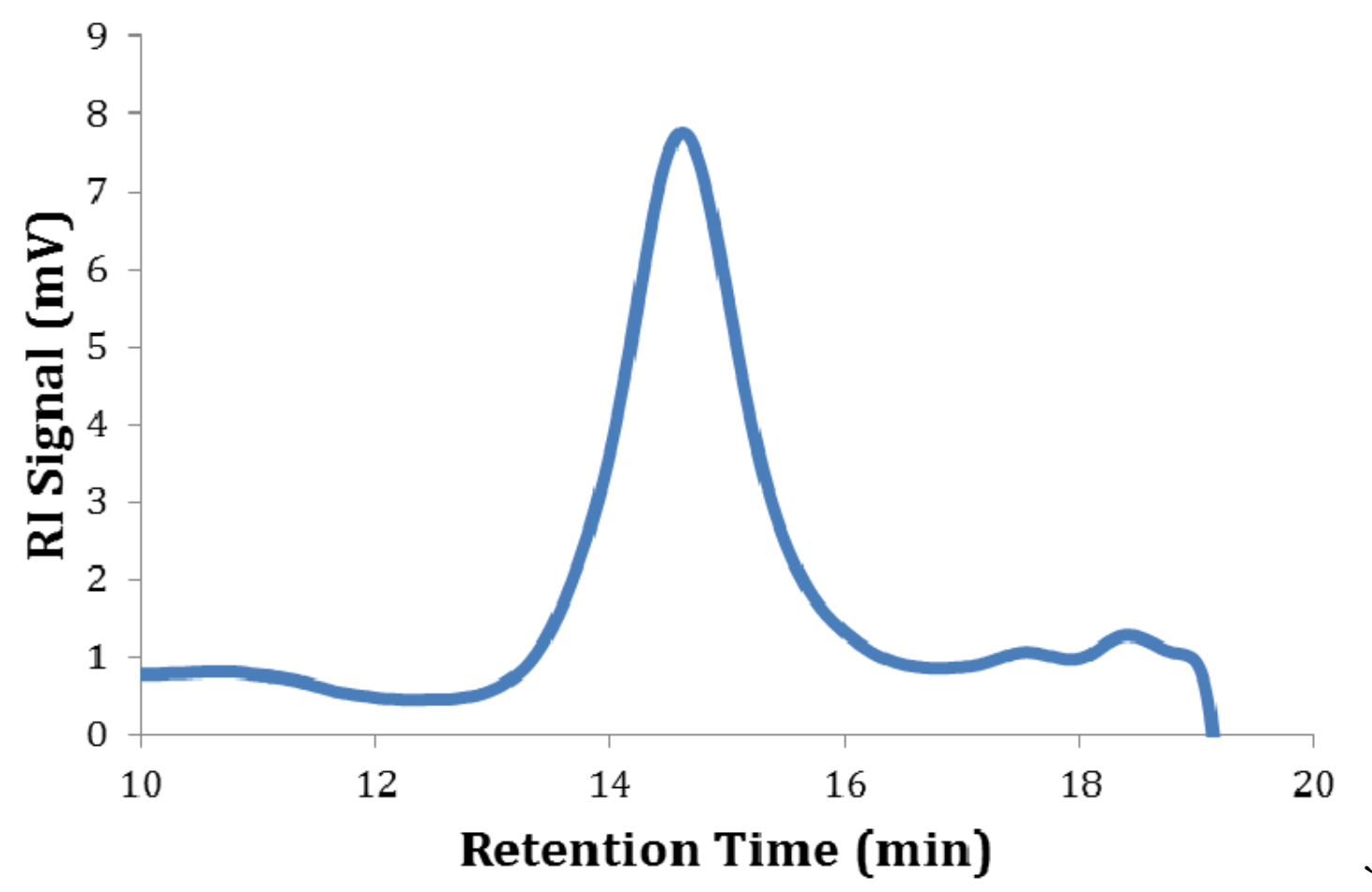

Figure S132. Table S1, entry 1: Polymerization of 100 equivalents of L-lactide with the catalyst in the reduced state; $\mathrm{M}_{\mathrm{n}}=12217 \mathrm{Da}, \mathrm{M}_{\mathrm{w}}=15882 \mathrm{Da}, \mathrm{PDI}=1.30$.

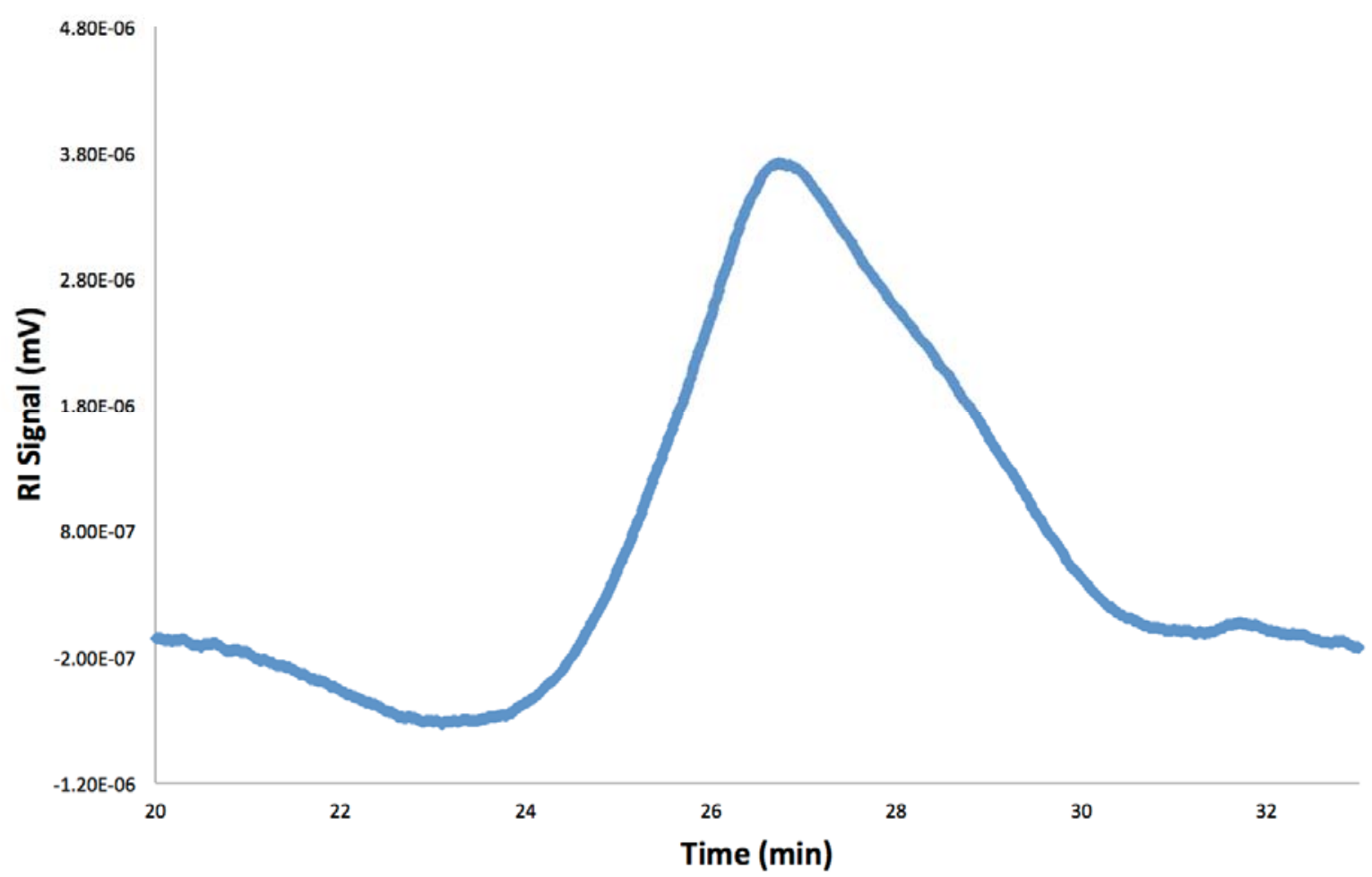

Figure S133. Table S2, entry 3: Acetone precipitate from selective precipitation of PLA-PCHO; $\mathrm{M}_{\mathrm{n}}=12940 \mathrm{Da}, \mathrm{M}_{\mathrm{w}}=17210 \mathrm{Da}, \mathrm{PDI}=1.33$. 


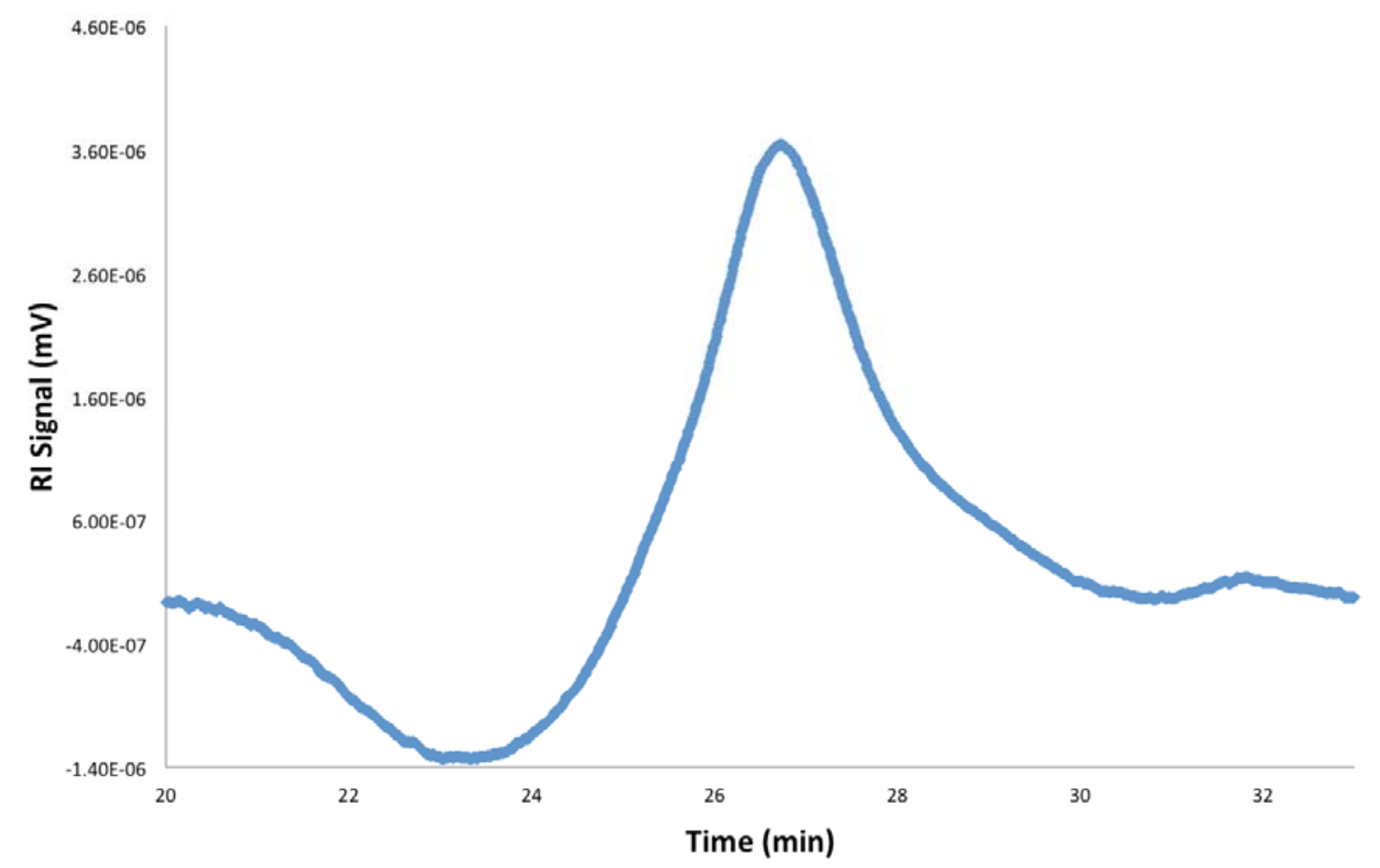

Figure S134. Table S2, entry 5: Hexanes precipitate from selective precipitation of PLA-PCHO; $\mathrm{M}_{\mathrm{n}}=12760 \mathrm{Da}, \mathrm{M}_{\mathrm{w}}=15950 \mathrm{Da}, \mathrm{PDI}=1.25$.

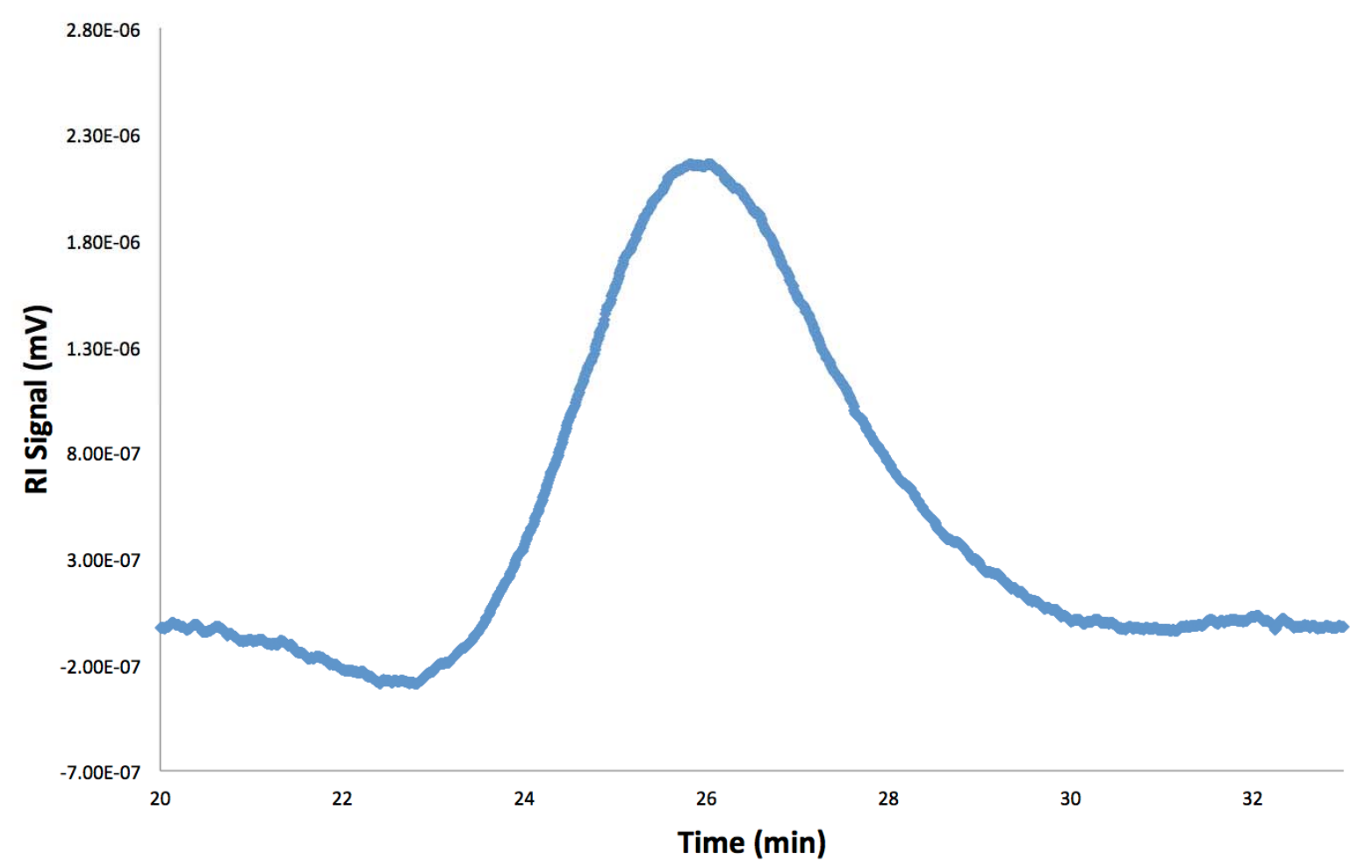

Figure S135. Table S3, entry 3: Acetone precipitate from selective precipitation of PLA-PCHOPLA; $\mathrm{M}_{\mathrm{n}}=17420 \mathrm{Da}, \mathrm{M}_{\mathrm{w}}=21427 \mathrm{Da}, \mathrm{PDI}=1.23$. 


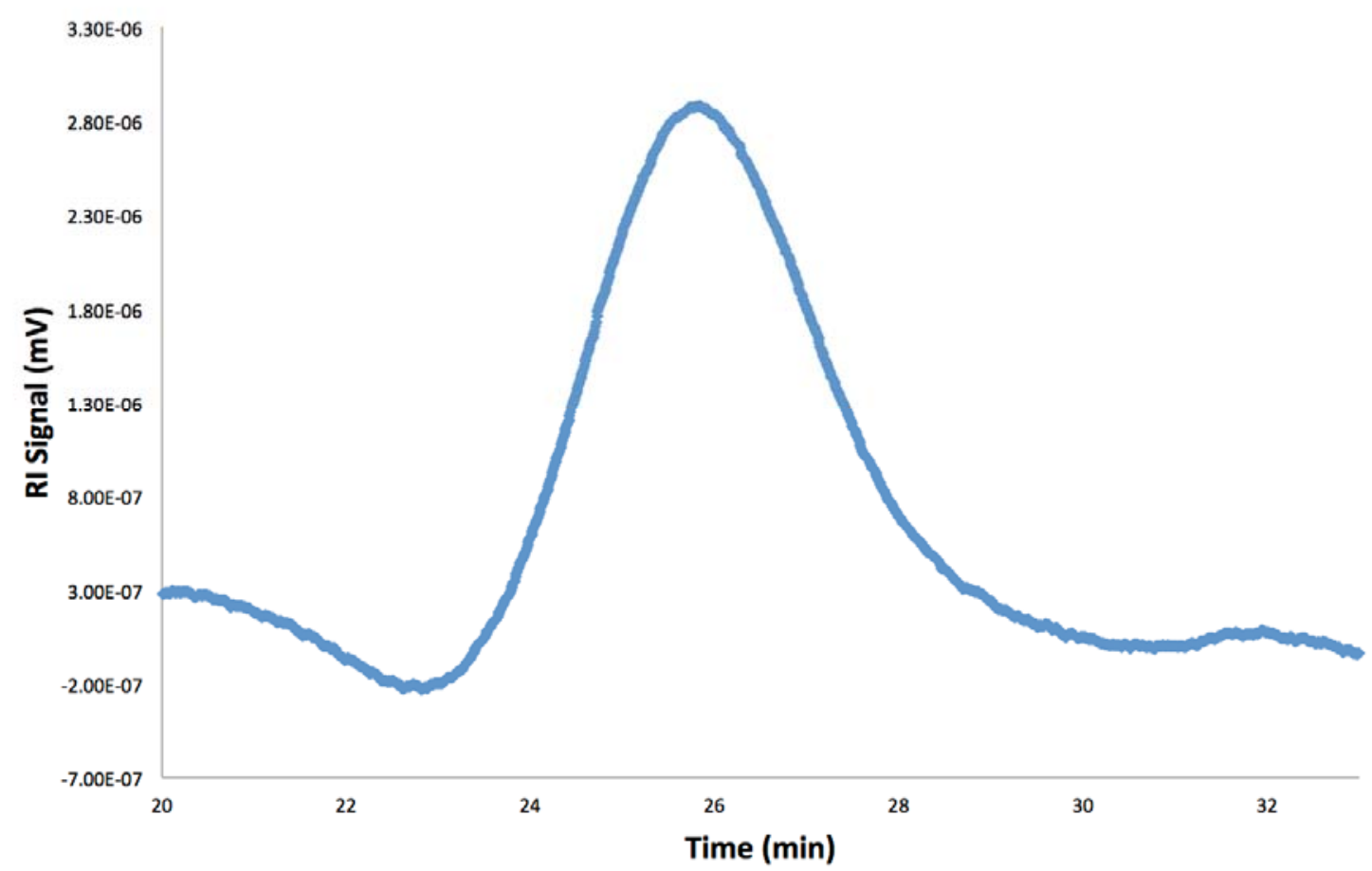

Figure S136. Table S3, entry 5: Hexanes precipitate from selective precipitation of PLA-PCHOPLA; $\mathrm{M}_{\mathrm{n}}=18140 \mathrm{Da}, \mathrm{M}_{\mathrm{w}}=25033 \mathrm{Da}, \mathrm{PDI}=1.38$.

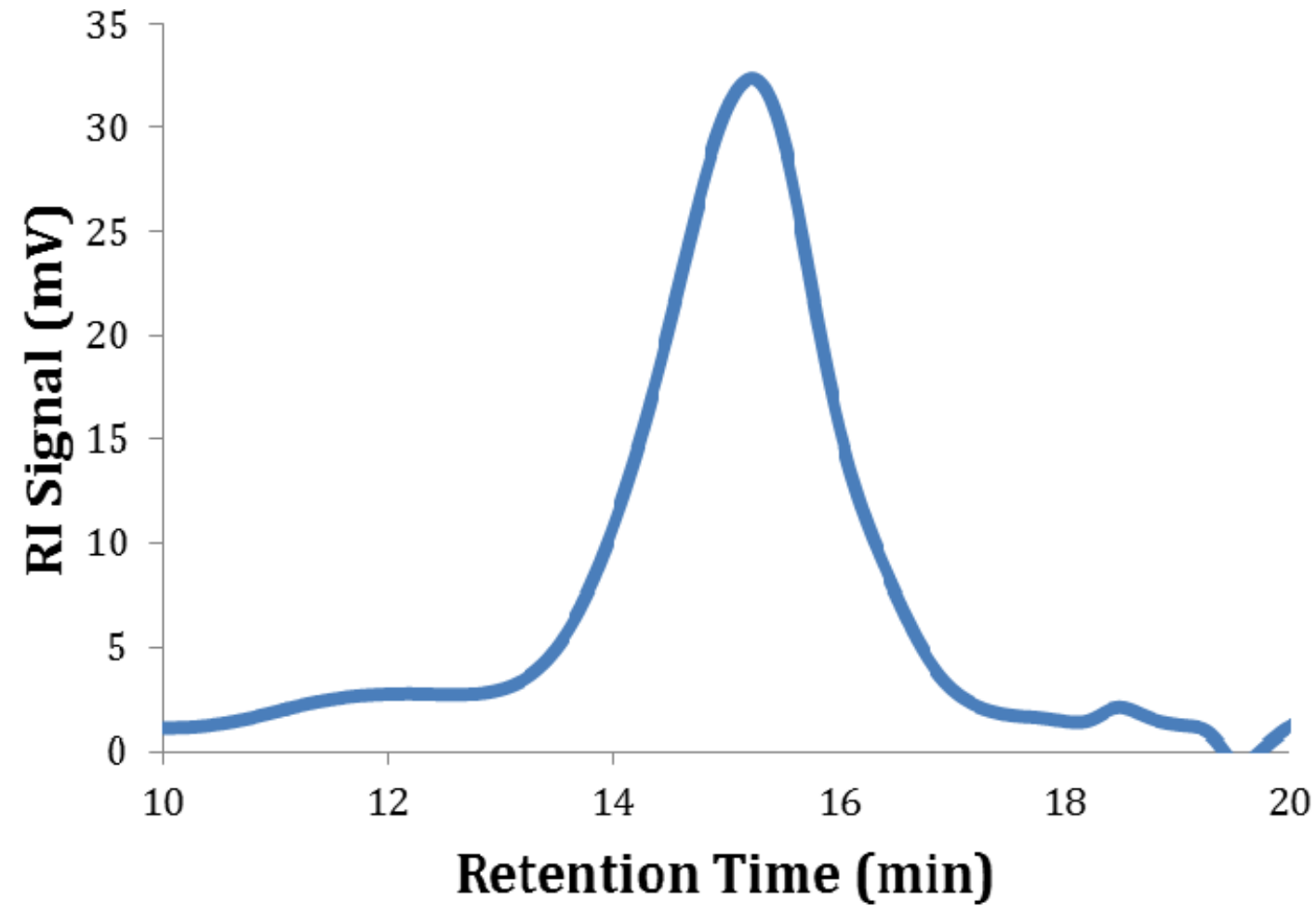

Figure S137. Table S1, entry 4: Polymerization of 100 equivalents of cyclohexene oxide with the catalyst in the oxidized state; $\mathrm{M}_{\mathrm{n}}=4046 \mathrm{Da}, \mathrm{M}_{\mathrm{w}}=5583 \mathrm{Da}, \mathrm{PDI}=1.38$. 


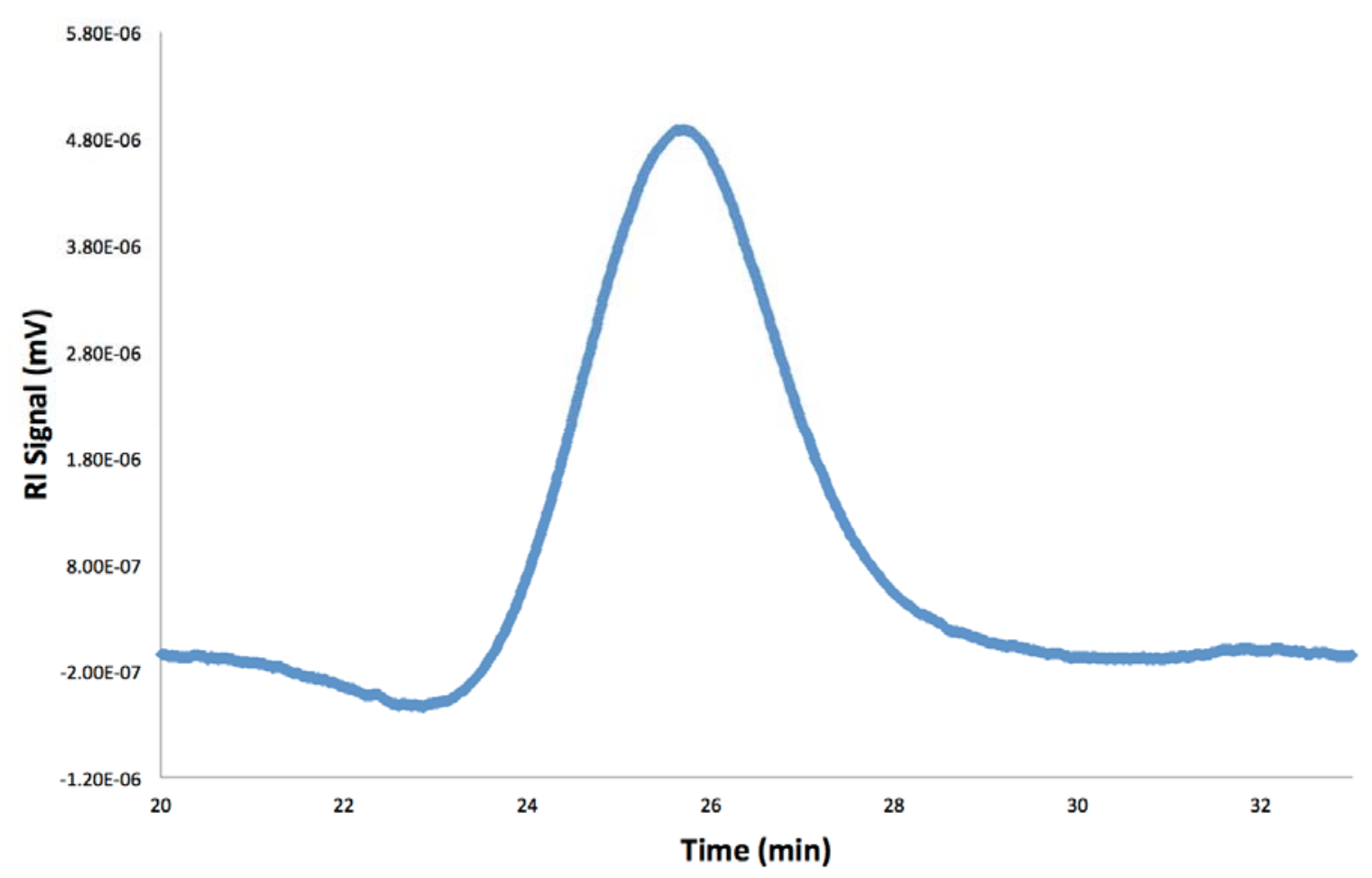

Figure S138. Table S4, entry 3: Acetone precipitate from selective precipitation of PCHO-PLA; $\mathrm{M}_{\mathrm{n}}=18560 \mathrm{Da}, \mathrm{M}_{\mathrm{w}}=27468 \mathrm{Da}, \mathrm{PDI}=1.48$.

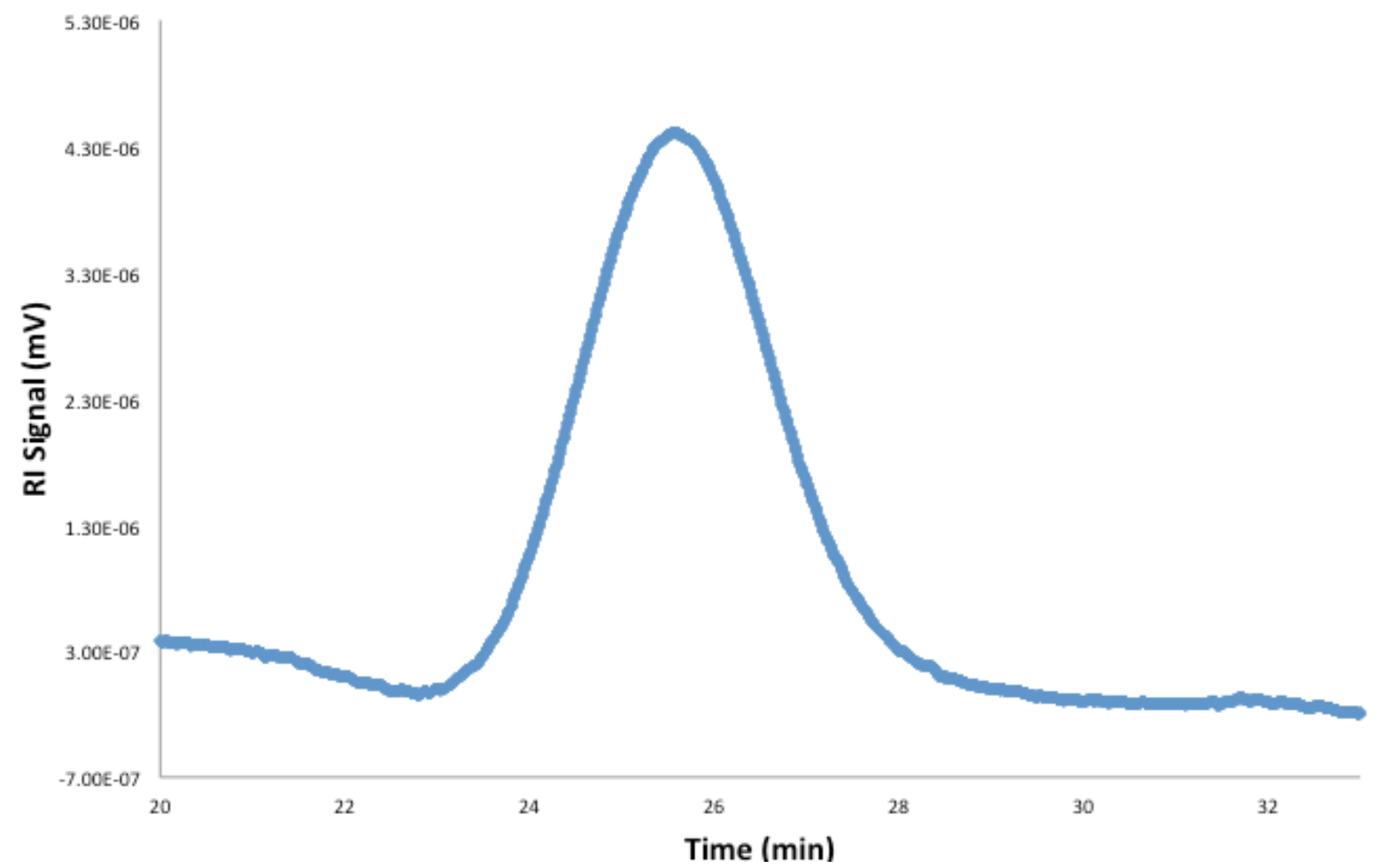

Figure S139. Table S4, entry 5: Hexanes precipitate from selective precipitation of PCHO-PLA; $\mathrm{M}_{\mathrm{n}}=15820 \mathrm{Da}, \mathrm{M}_{\mathrm{w}}=25312 \mathrm{Da}, \mathrm{PDI}=1.60$. 


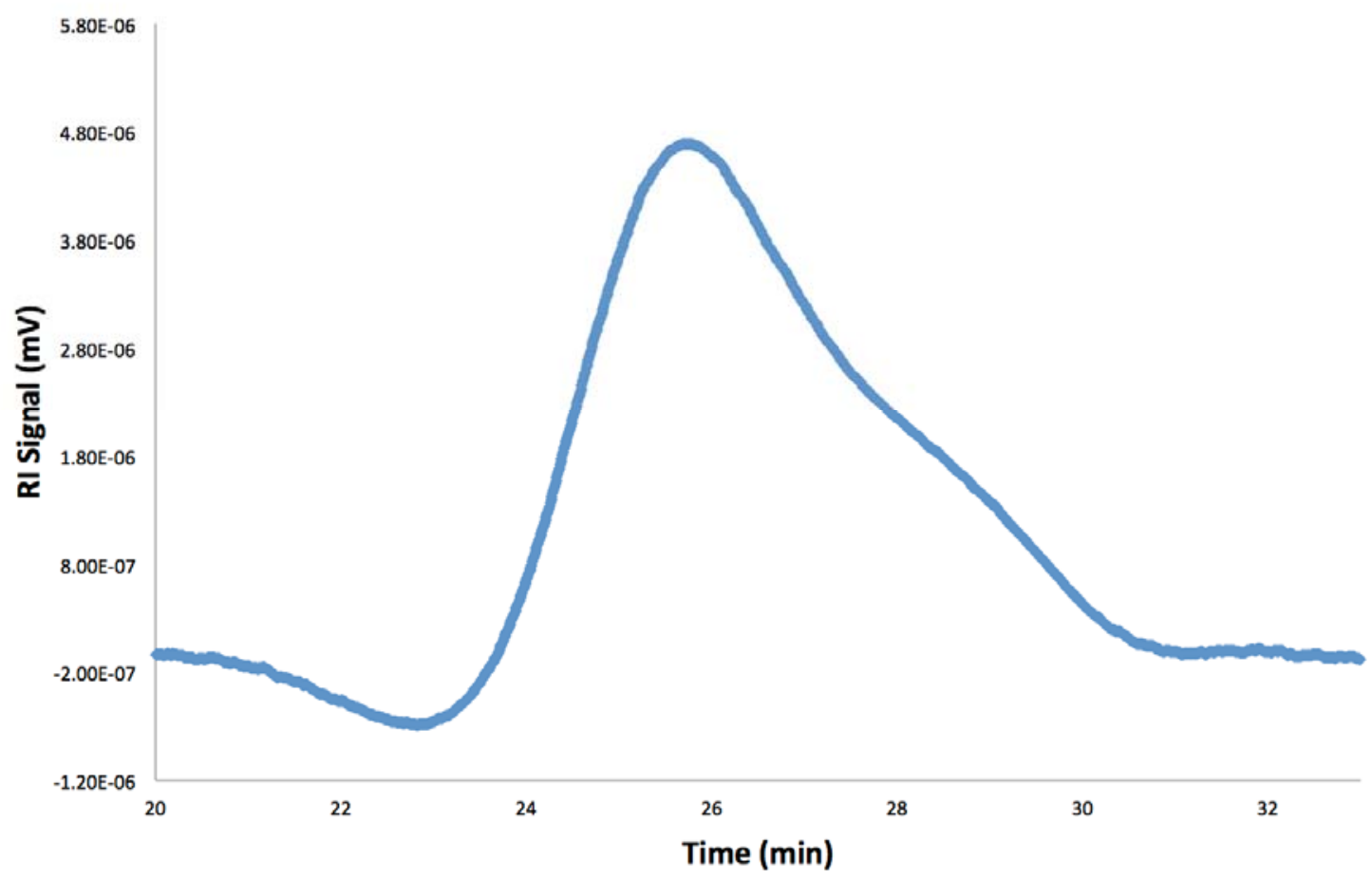

Figure S140. Table S5, entry 3: Acetone precipitate from selective precipitation of PCHO-PLAPCHO; $\mathrm{M}_{\mathrm{n}}=13990 \mathrm{Da}, \mathrm{M}_{\mathrm{w}}=20565 \mathrm{Da}, \mathrm{PDI}=1.47$.

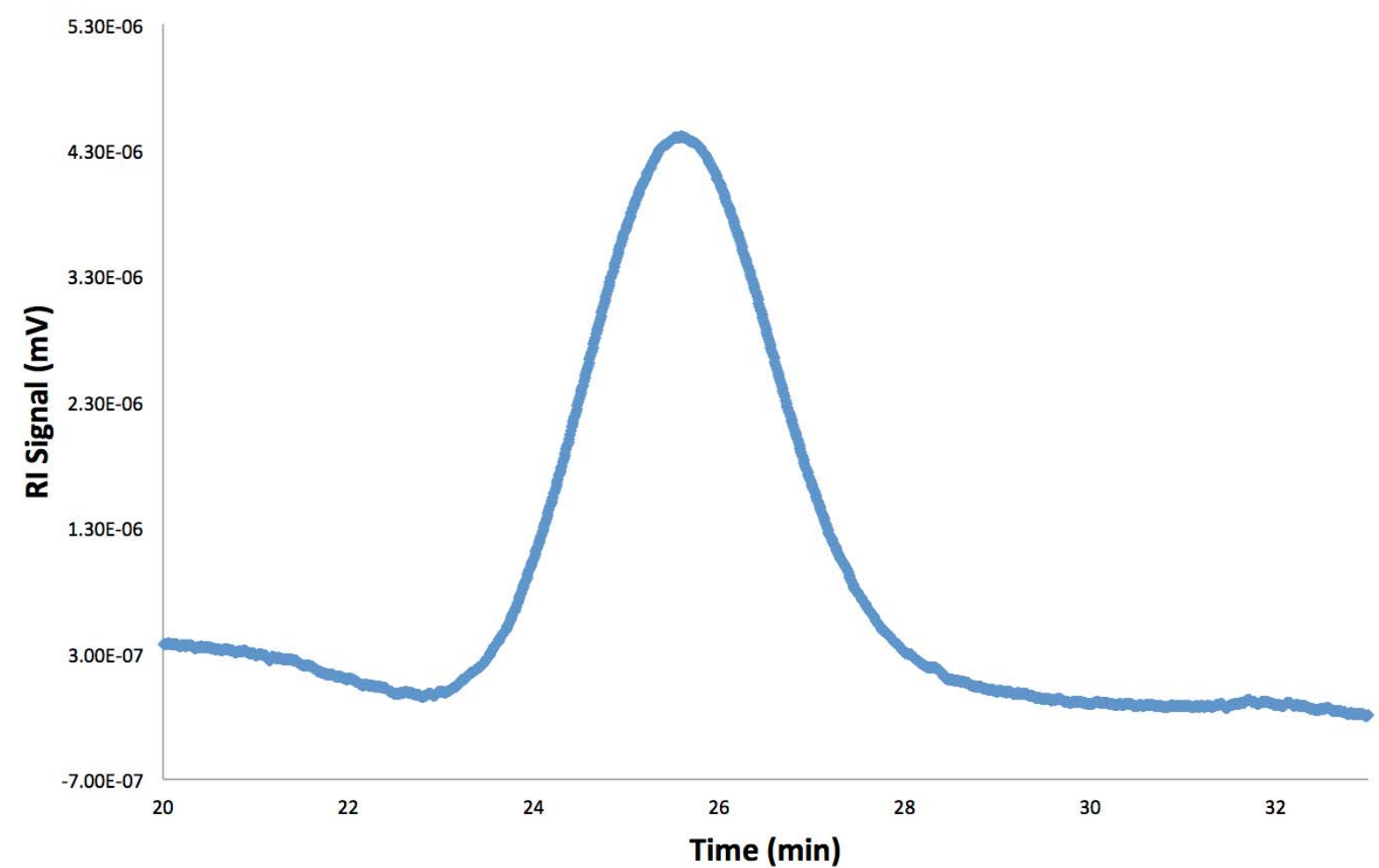

Figure S141. Table S5, entry 5: Hexanes precipitate from selective precipitation of PCHO-LAPCHO; $\mathrm{M}_{\mathrm{n}}=16790 \mathrm{Da}, \mathrm{M}_{\mathrm{w}}=25017 \mathrm{Da}, \mathrm{PDI}=1.49$. 


\section{Thermal Gravimetric Analysis}

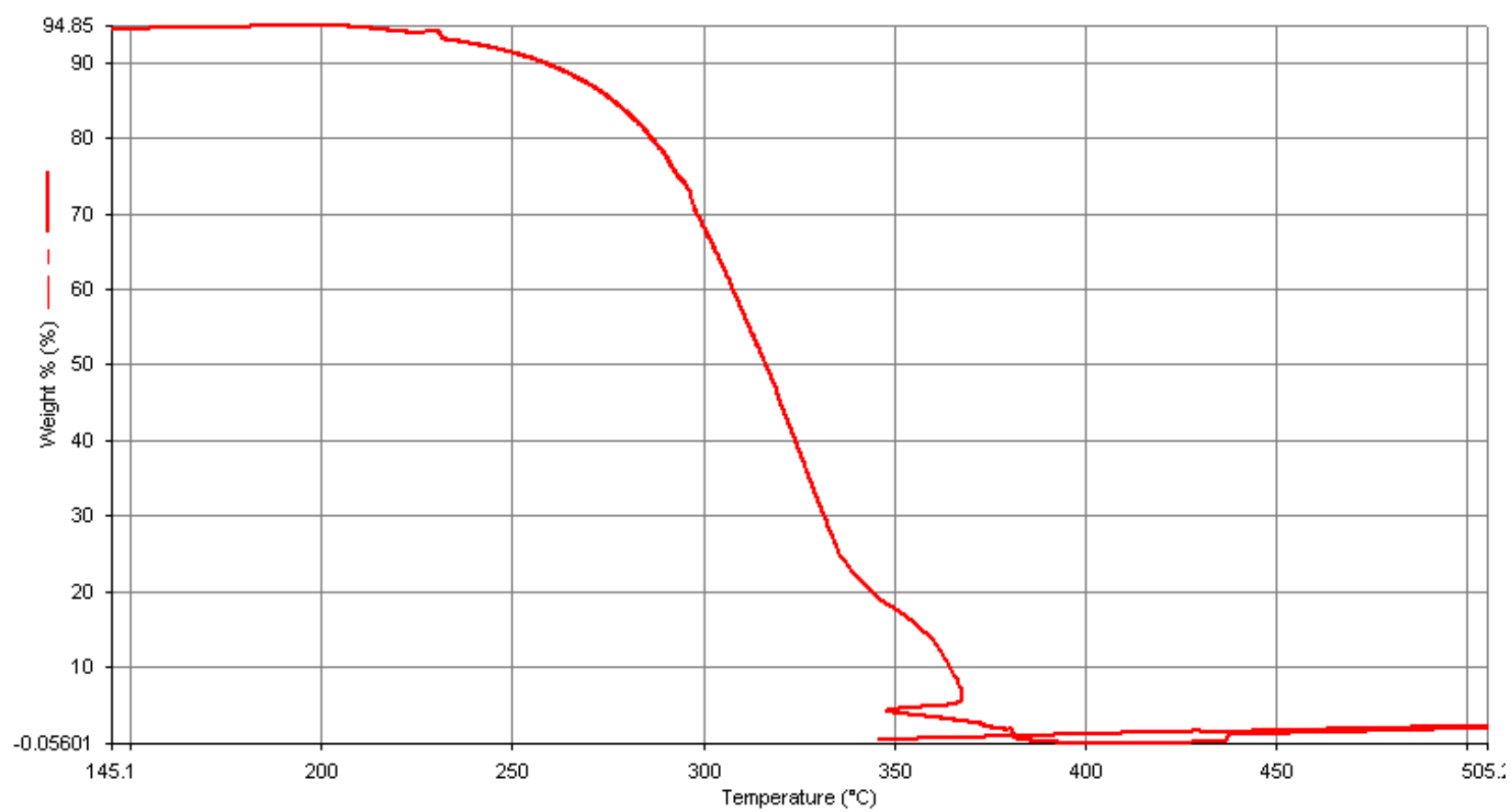

Figure 142. Thermal decomposition of the PLA-PCHO diblock copolymer obtained by sequential addition.

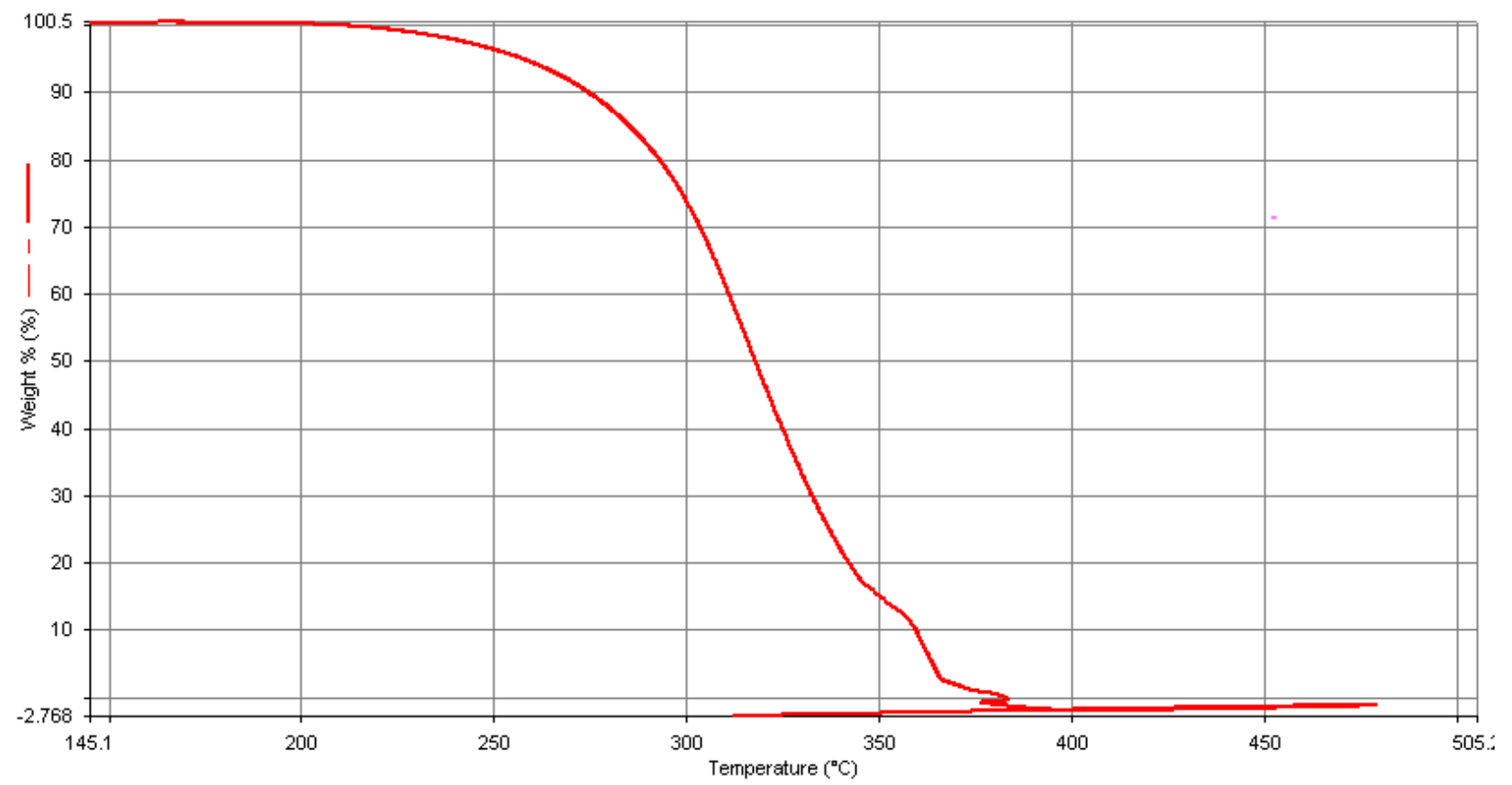

Figure 143. Thermal decomposition of the PLA-PCHO-PLA triblock copolymer obtained by sequential addition. 


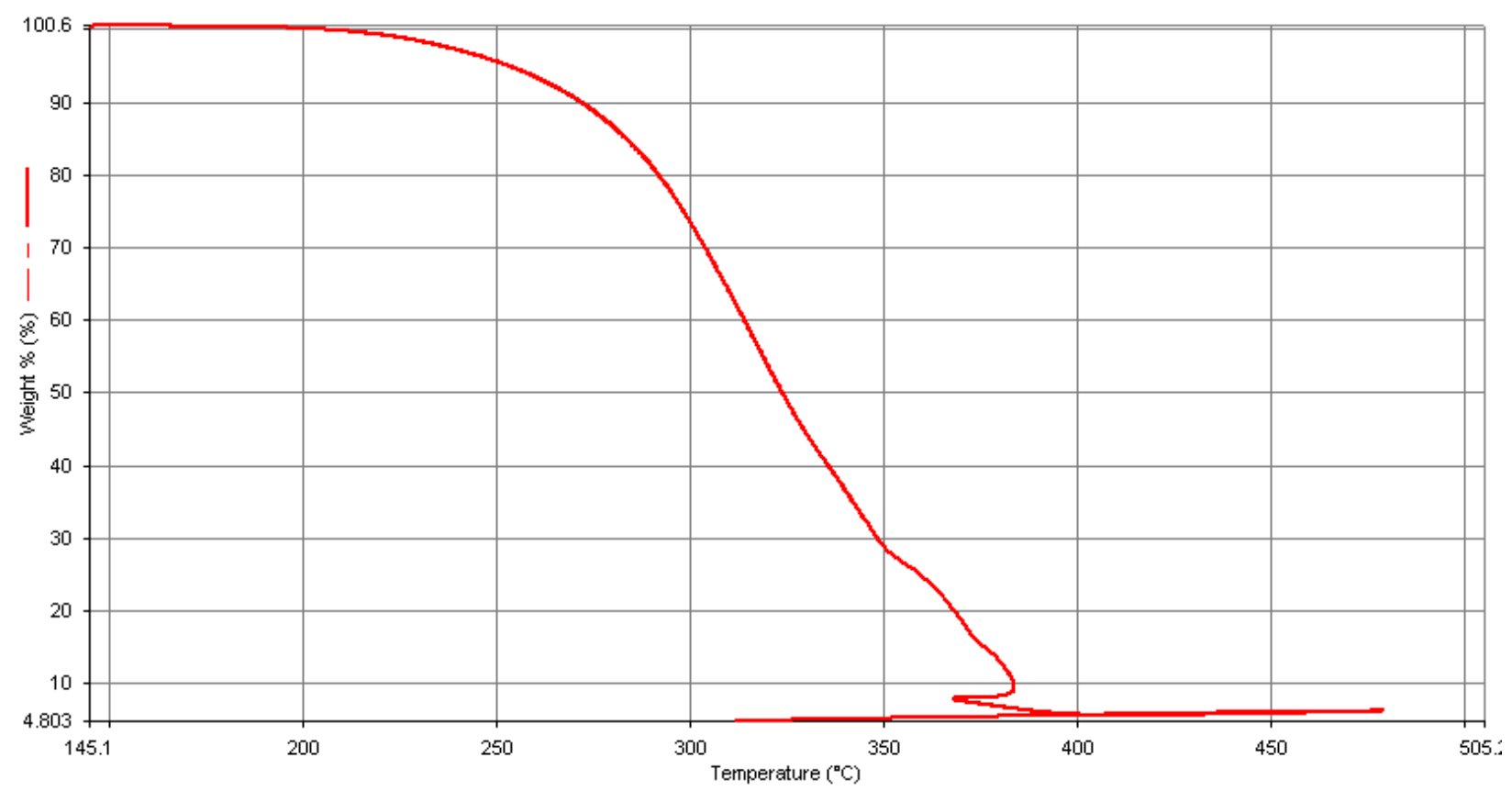

Figure 144. Thermal decomposition of the PCHO-PLA diblock copolymer obtained by sequential addition.

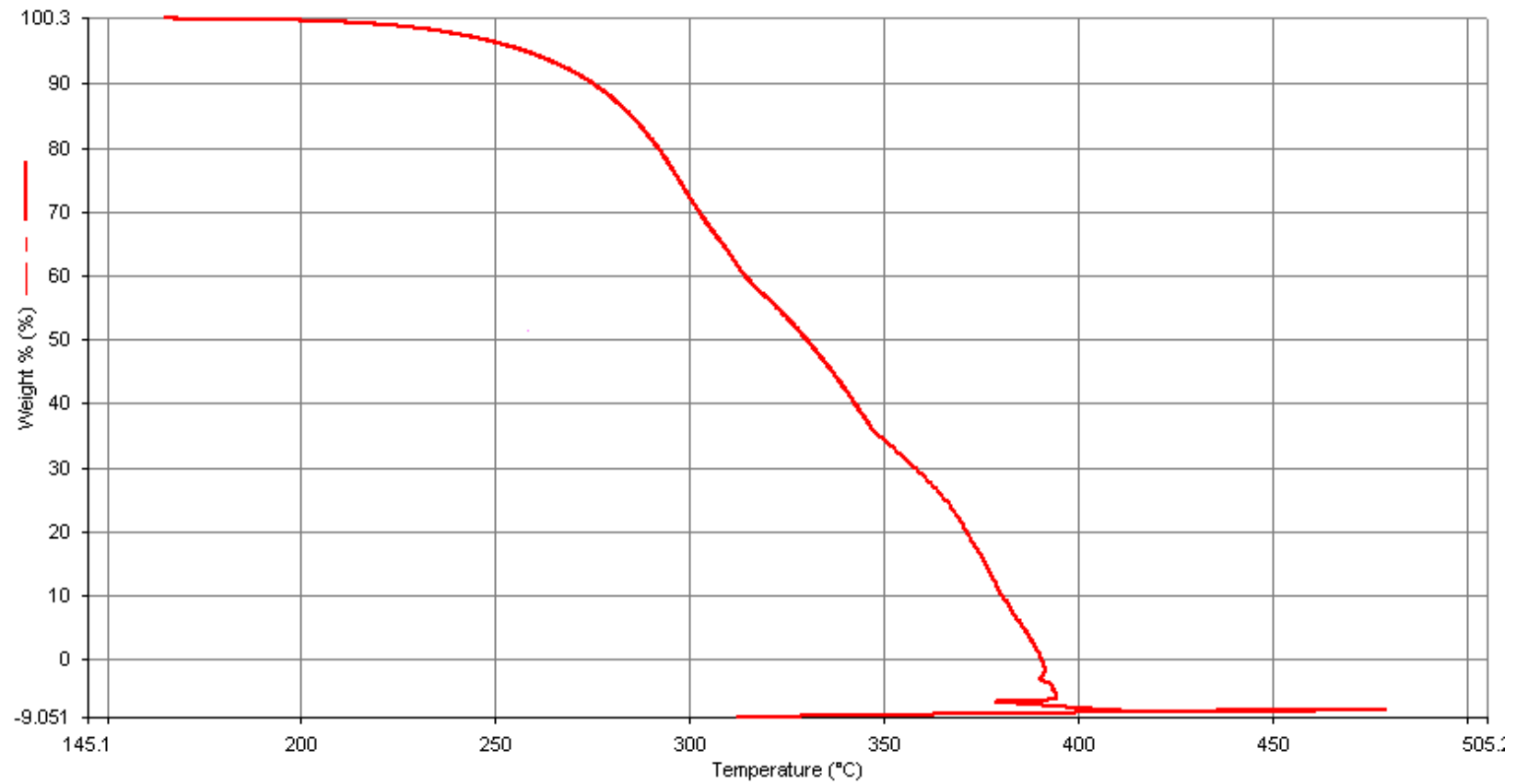

Figure 145. Thermal decomposition of the PCHO-PLA-PCHO triblock copolymer obtained by sequential addition. 


\section{Differential Scanning Calorimetry}

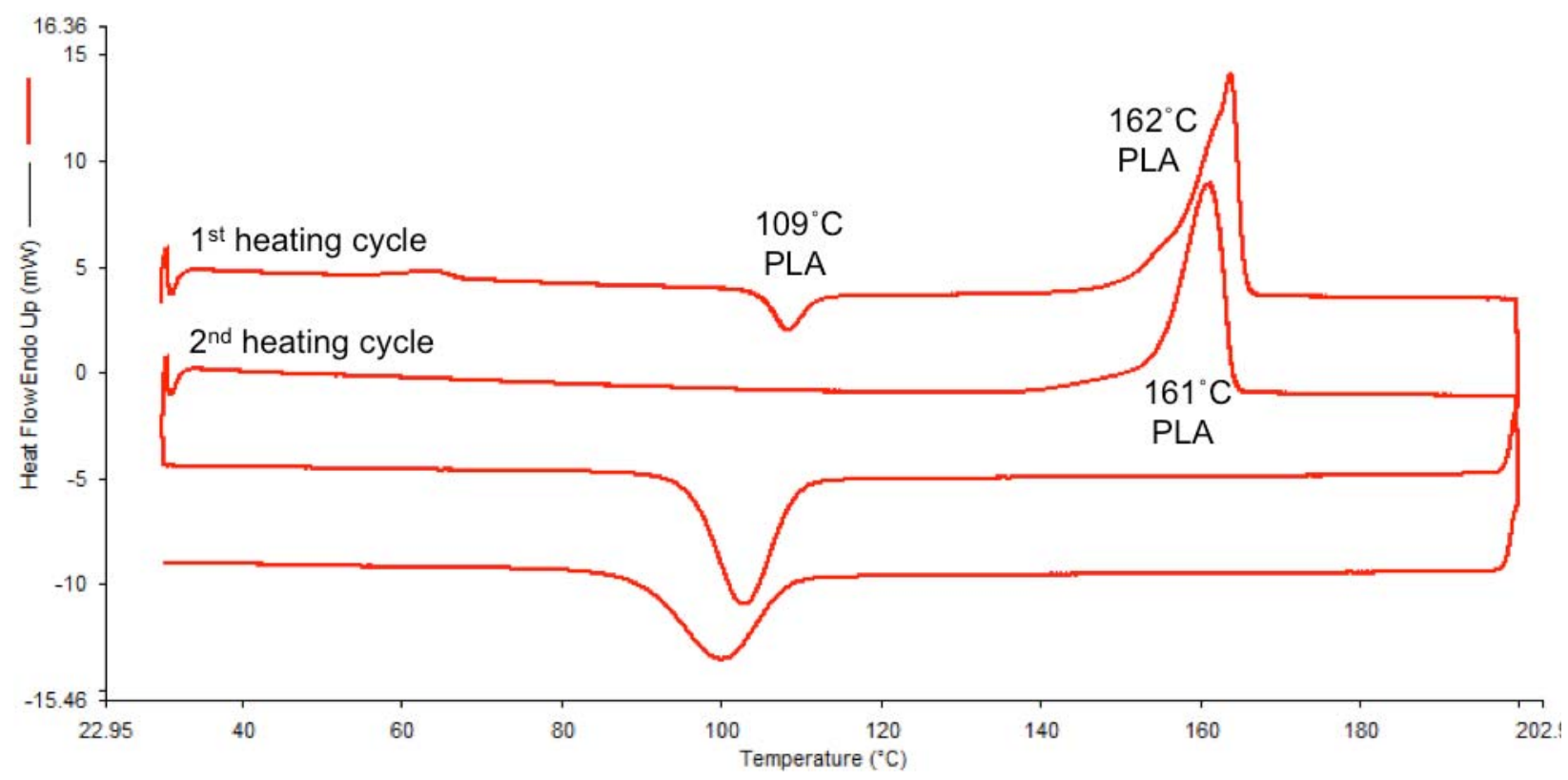

Figure 146. DSC analysis of a PLA homopolymer (7 mg).

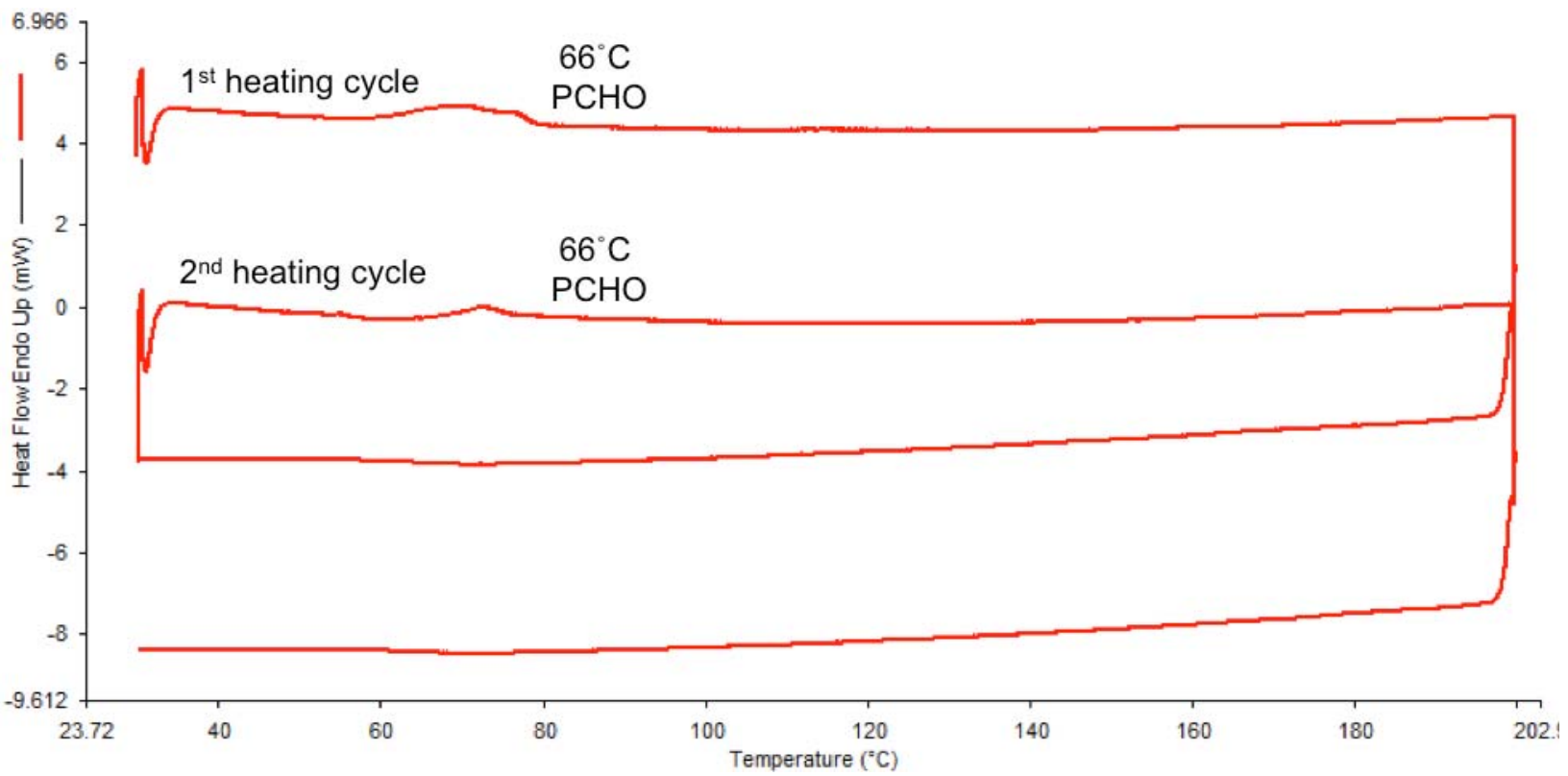

Figure 147. DSC analysis of a PCHO homopolymer (8 mg). 


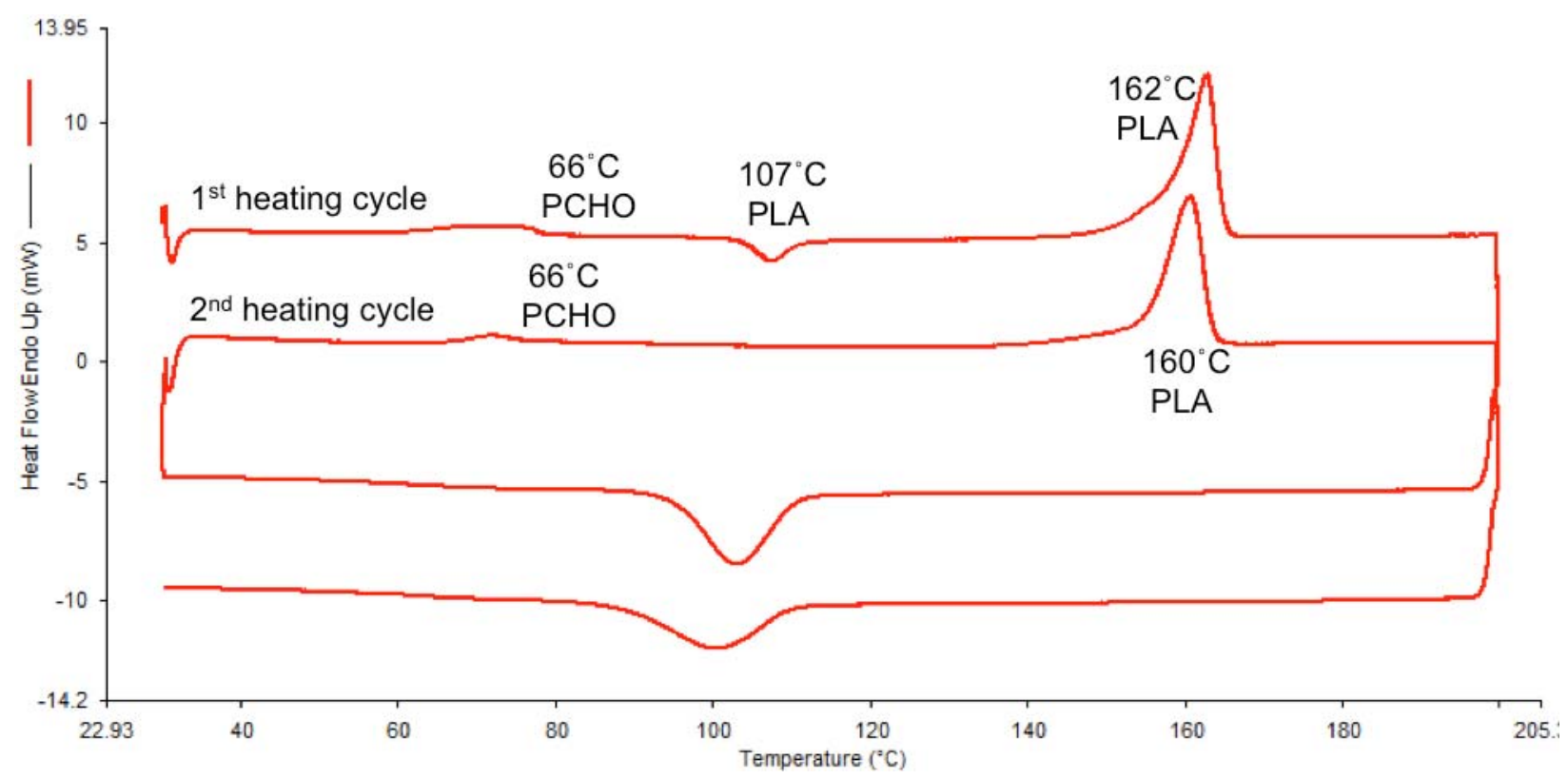

Figure 148. DSC analysis of a physical mixture of PLA (4 mg) and PCHO (6 mg) homopolymers.

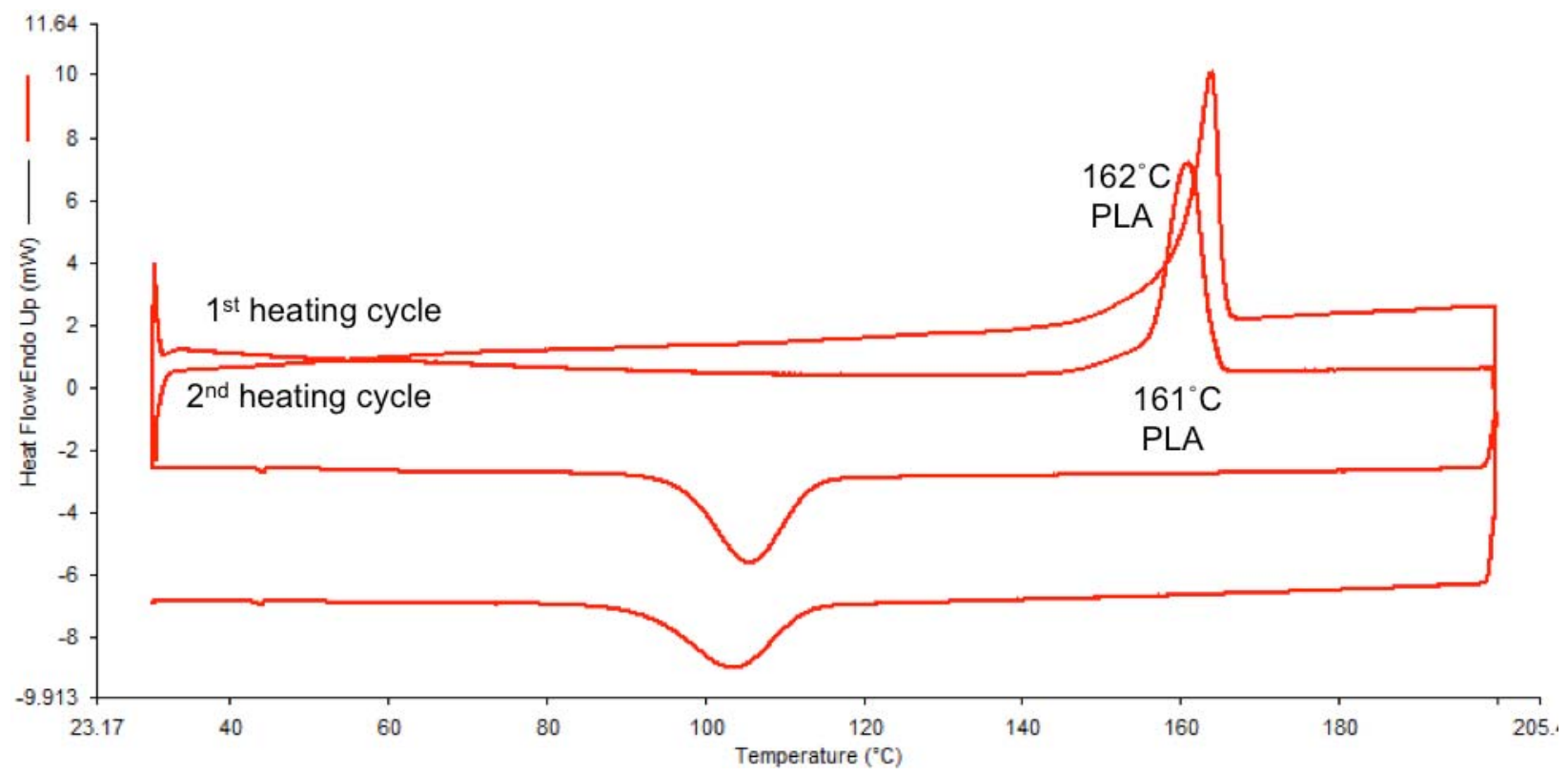

Figure 149. DSC analysis of a PLA-PCHO copolymer obtained by sequential addition ( $9 \mathrm{mg})$. 


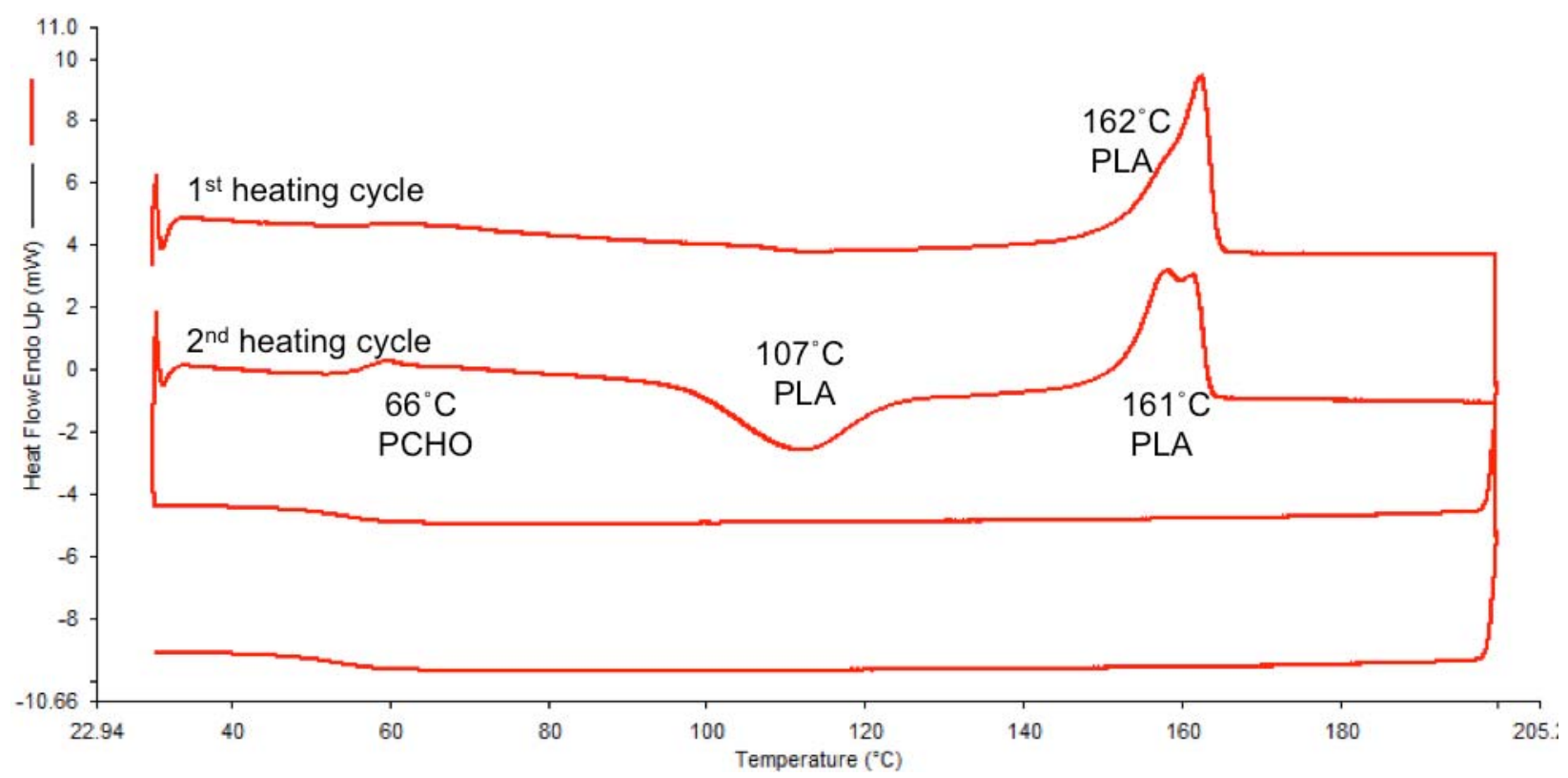

Figure 150. DSC analysis of a PLA-PCHO-PLA copolymer obtained by sequential addition (7 $\mathrm{mg}$ ).

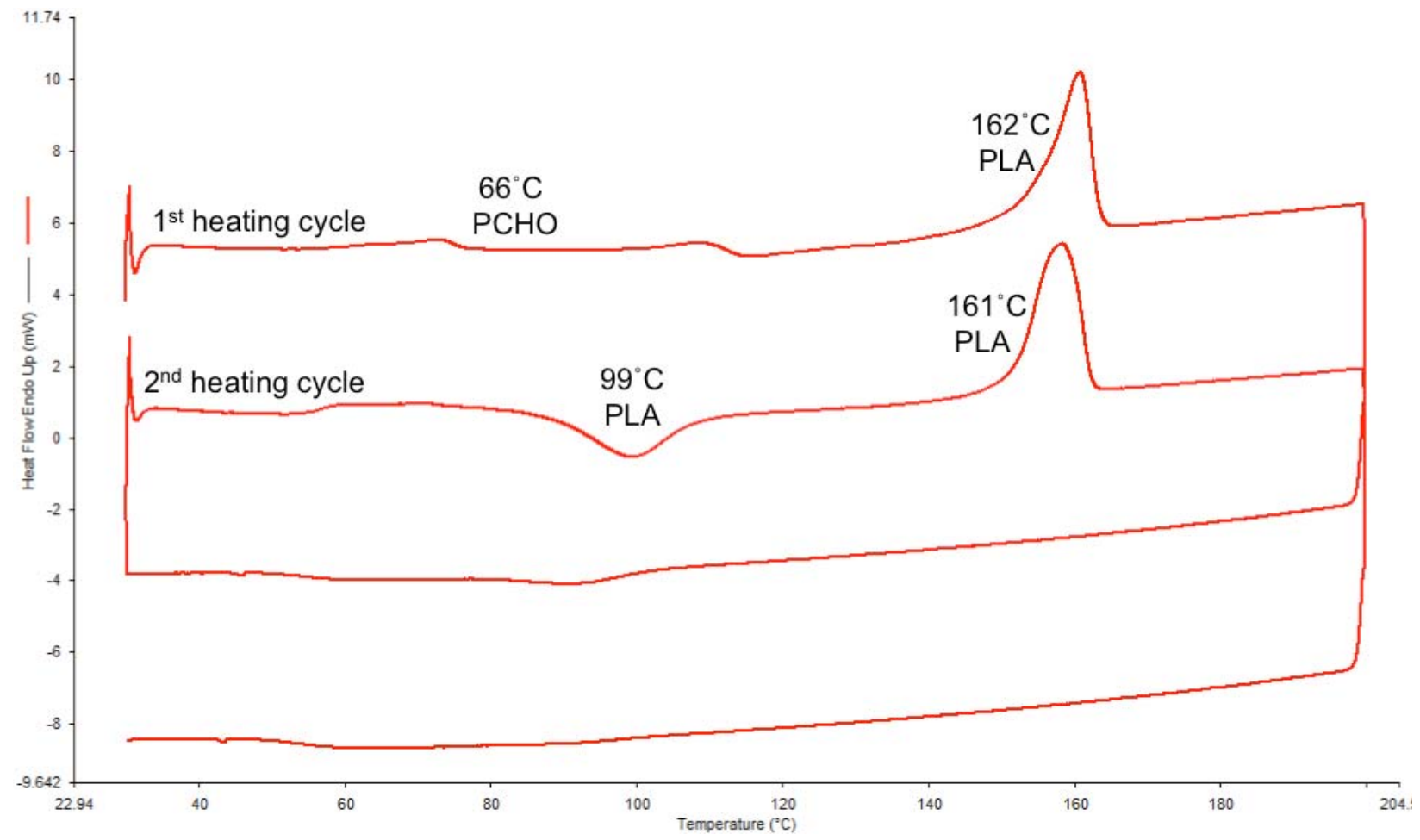

Figure 151. DSC analysis of a PCHO-PLA copolymer obtained by sequential addition (7 mg). 


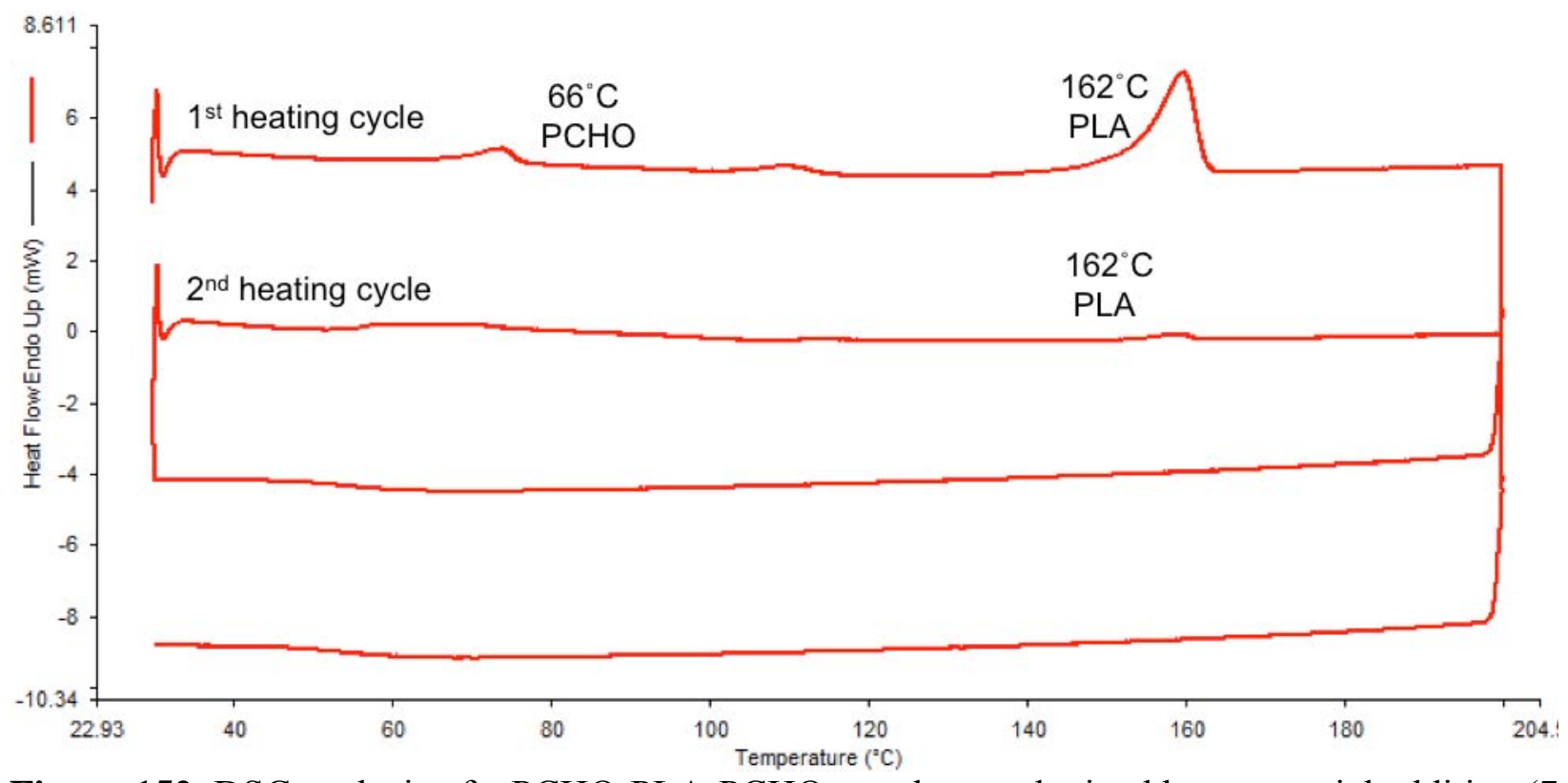

Figure 152. DSC analysis of a PCHO-PLA-PCHO copolymer obtained by sequential addition (7 $\mathrm{mg})$. 